Linköping Studies in Science and Technology

Dissertations. No. 1702

\title{
Thermal Issues in Testing of Advanced Systems on Chip
}

By

Nima Aghaee

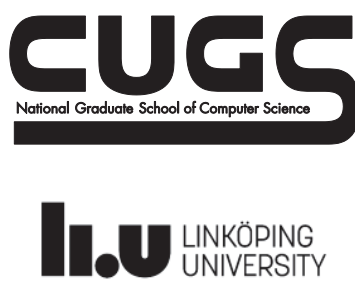

Department of Computer and Information Science

Linköping University

SE-581 83 Linköping, Sweden

Linköping 2015 
Copyright (C) 2015 Aghaee Ghaleshahi, Nima ISBN 978-91-7685-949-0

ISSN 0345-7524

Printed by LiU-Tryck 2015

URL: http://urn.kb.se/resolve?urn=urn:nbn:se:liu:diva-120798 


\section{Abstract}

Many cutting-edge computer and electronic products are powered by advanced Systems-on-Chip (SoC). Advanced SoCs encompass superb performance together with large number of functions. This is achieved by efficient integration of huge number of transistors. Such very large scale integration is enabled by a core-based design paradigm as well as deepsubmicron and 3D-stacked-IC technologies. These technologies are susceptible to reliability and testing complications caused by thermal issues. Three crucial thermal issues related to temperature variations, temperature gradients, and temperature cycling are addressed in this thesis.

Existing test scheduling techniques rely on temperature simulations to generate schedules that meet thermal constraints such as overheating prevention. The difference between the simulated temperatures and the actual temperatures is called temperature error. This error, for past technologies, is negligible. However, advanced SoCs experience large errors due to large process variations. Such large errors have costly consequences, such as overheating, and must be taken care of. This thesis presents an adaptive approach to generate test schedules that handle such temperature errors.

Advanced SoCs manufactured as 3D-stacked-ICs experience large temperature gradients. Temperature gradients accelerate certain early-life defect mechanisms. These mechanisms can be accelerated using gradientbased, burn-in like operations so that the defects are detected before shipping. Moreover, temperature gradients exacerbate some delay-related defects. In order to detect such defects, testing must be performed when appropriate temperature-gradients are enforced. Schedule-based techniques that enforces the temperature-gradients for burn-in like operations and delay testing are proposed in this thesis. 


\begin{abstract}
The last thermal issue addressed by this thesis is related to temperature cycling. Temperature cycling test procedures are usually applied to safetycritical systems to detect cycling-related early-life failures. Such failures affect advanced SoCs, particularly through-silicon-via structures in 3Dstacked-ICs. An efficient schedule-based cycling-test technique that combines cycling acceleration with testing is proposed in this thesis. The proposed technique fits into existing $3 \mathrm{D}$ testing procedures and does not require temperature chambers. Therefore, the overall cycling acceleration and testing cost can be drastically reduced.

All the proposed techniques have been implemented and evaluated with extensive experiments based on ITC'02 benchmarks as well as a number of 3D stacked ICs. Experiments show that the proposed techniques work effectively and reduce the test costs. We have also developed a fast temperature simulation technique based on a closed-form solution for the temperature equations. Experiments demonstrate that the proposed simulation technique reduces the test schedule generation time by more than half.
\end{abstract}




\section{Populärvetenskaplig sammanfattning}

Många banbrytande dator- och elektronikprodukter drivs av avancerade System-on-Chip (SoC). Avancerade SoCs har enastående prestanda tillsammans med ett stort antal funktioner. Detta uppnås genom effektiv integrering av ett stort antal transistorer. En sådan storskalig integration möjliggörs av ett kärnbaserat designparadigm samt djup submicron och 3D-stacked-IC teknik. Dessa teknologier är känsliga för tillförlitlighet och testkomplikationer orsakade av termiska problem. Tre viktiga termiska frågor som berör temperaturvariationer, temperaturgradienter och temperaturcykler behandlas i denna avhandling.

Befintliga testschemaläggningstekniker förlitar sig på temperatursimuleringar för att generera scheman som uppfyller termiska begränsningar. Skillnaden mellan de simulerade temperaturerna och de faktiska temperaturerna är ett fel. Detta fel, för tidigare tekniker, är försumbart. Men avancerade SoCs upplever stora fel på grund av stora processvariationer. Sådana stora fel har kostsamma följder, så som överhettning, och måste tas om hand.

Avancerade SoCs tillverkade som 3D-stacked-IC upplever stora temperaturgradienter. Temperaturgradienter påskyndar uppkomsten av vissa defekta mekanismer när produkten är ny. Dessa mekanismer kan artificiellt påskyndas genom att tillämpa gradienter så att motsvarande fel upptäcks i tid. Dessutom förvärrar temperaturgradienter vissa fördröjningsrelaterade defekter. För att upptäcka sådana defekter måste testen utföras när lämpliga temperaturgradienter appliceras.

Den sista värmefrågan som behandlas i denna avhandling är relaterad till temperaturcykling. Temperaturcyklingstester används för att detektera cykelrelaterade fel tidigt. Sådana fel påverkar avancerade SoCs, särskilt through-silicon-via-strukturer i 3D-stacked-IC. Befintliga 
temperaturcyklings-testmetoder är för dyra för 3D-stacked-IC och därmed måste nya billigare tekniker utvecklas.

Denna avhandling föreslår effektiva schemabaserade lösningar för termiska problem så som diskuteras ovan. Dessa inkluderar termiska testoch tillförlitlighetsproblem i samband med processvariation, temperaturgradienter och temperaturvariationer. En snabb temperatursimuleringsteknik föreslås $\mathrm{i}$ denna avhandling. Omfattande experiment har visat effektiviteten av dessa föreslagna tekniker. 


\section{Acknowledgements}

I would like to express my sincere gratitude and appreciation to my advisors Professor Zebo Peng and Professor Petru Eles. I am thankful for the opportunity, support, education, and training that they have provided throughout my doctoral studies.

I would like to thank and express my appreciations to the Swedish National Graduate School in Computer Science, CUGS, for funding and supporting my research and studies.

I cannot forget the impact the high quality doctoral courses had on my professional life by offering new perspectives. Even though I cannot name them one by one I must thank professors and teachers that offered them.

Many thanks go to my friends and other employees at the embedded systems laboratory, ESLAB, and at the department of computer and information science, IDA, (including my colleagues, administration, technical sections, etc.) for the pleasant and supportive work place that they have created.

All these support would have not worked without the extraordinary support and motivation from my parents and siblings. Thank you all!

Nima Aghaee Ghaleshahi

Linköping, September 2015 
Please note that those copies of this thesis that are printed by LiU-Tryck are in grayscale except for pages 8,161, and 171. All the full color figures can be found in the electronic copy. 


\section{Contents}

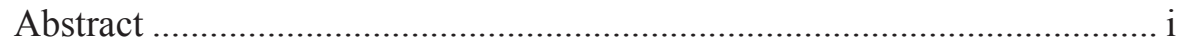

Populärvetenskaplig sammanfattning ....................................................... iii

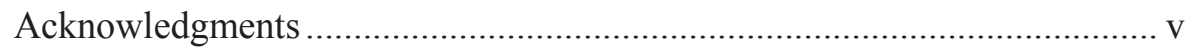

Chapter 1 Introduction 1

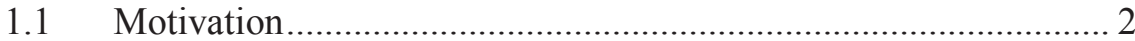

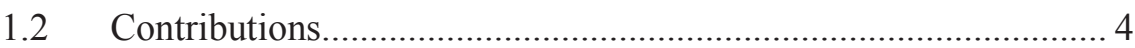

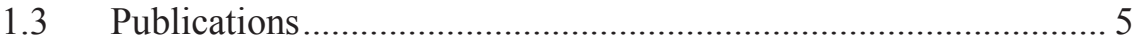

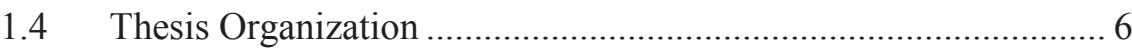

Chapter 2 Preliminaries $\quad 7$

$2.1 \quad$ Temperature Related Defects .................................................... 7

2.1.1 Temperature Dependent Defects........................................... 7

2.1.2 Early Life Failures ………………….................................. 9

2.1.3 Delay Faults ....................................................................... 10

2.2 Core-Based SoC Testing........................................................... 10

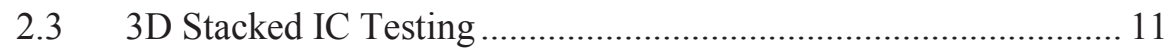

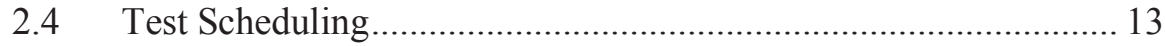

2.5 Test Power and Temperature .................................................... 16

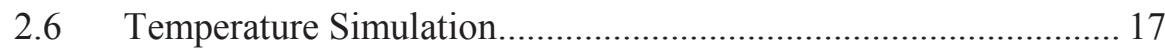

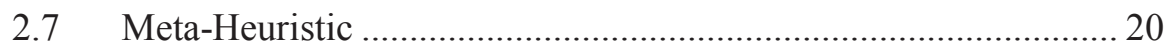

2.7.1 Motivational Example .................................................................. 20 


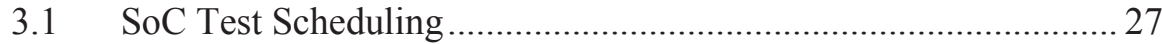

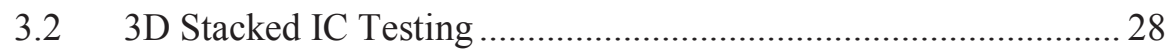

3.3 Temperature-Aware Test Scheduling …………………………... 30

3.4 Process Variation Effects on Power and Temperature.................... 33

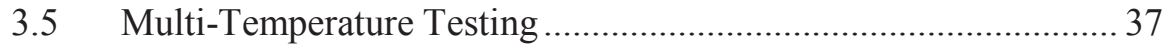

3.6 Temperature Gradients and Burn-In ............................................. 40

3.7 Testing for Delay-Related Defects................................................. 41

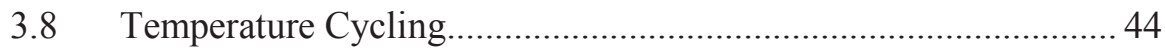

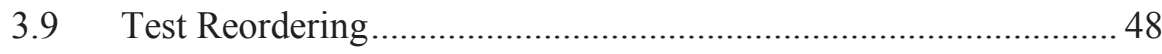

Chapter 4 Process-Variation Aware SoC Test Scheduling Techniques 51

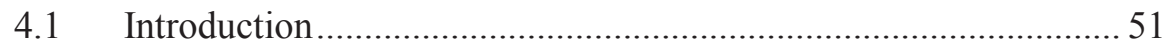

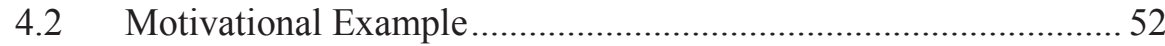

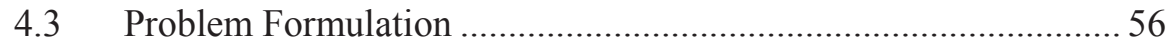

4.4 Temperature Error Model .............................................................5 59

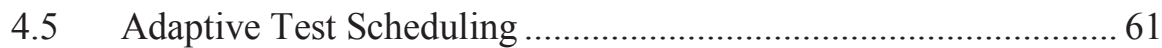

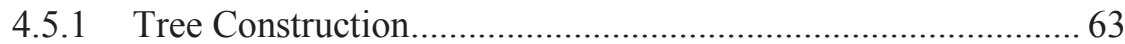

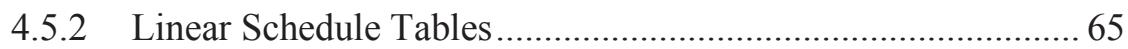

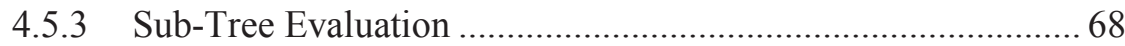

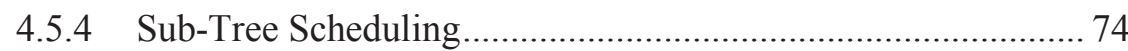

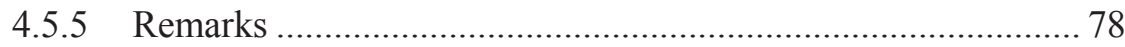

4.6 A Fast Temperature Simulation Approach ……………………..... 79

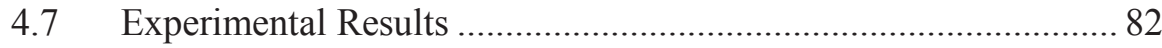

4.7.1 Fast Temperature Simulation Approach …………………........ 82

4.7.2 Adaptive Test Scheduling Technique ....................................... 84

4.8 Adaptive Multi-Temperature Testing ………………………….... 88 


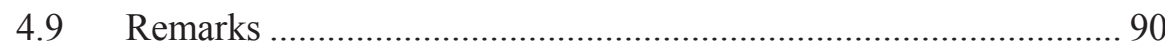

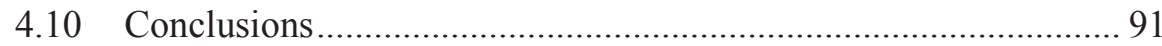

4.11 Notations and Abbreviations ......................................................... 93

Chapter 5 Temperature-Gradient Based Burn-In and Test Scheduling 97

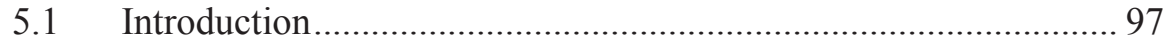

5.1.1 Test for Early-Life Failures ..................................................... 97

5.1.2 Test for Delay Faults................................................................. 99

5.2 Temperature-Gradient Based Burn-In .......................................... 101

5.2.1 Motivation and Problem Description......................................... 101

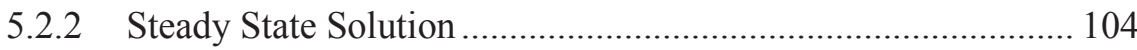

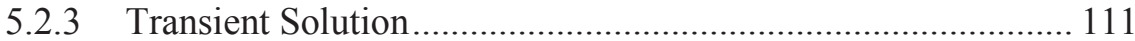

5.2.4 Transient-Based Heuristic.................................................... 115

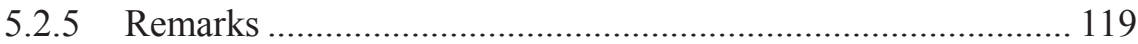

5.2.6 Experimental Results ............................................................. 121

$5.3 \quad$ Temperature-Gradient Based Test ................................................... 123

5.3.1 Straightforward Algorithm....................................................... 123

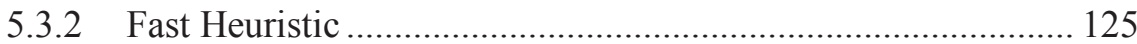

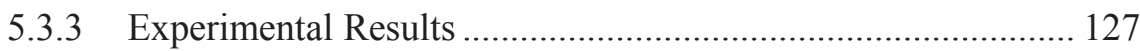

$5.4 \quad$ Temperature-Map Ordering .......................................................... 129

5.4.1 Map Ordering Technique...................................................... 129

5.4.2 Experimental Results ............................................................. 133

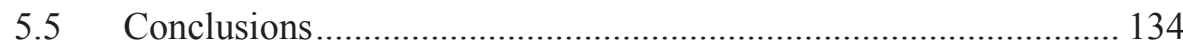

5.6 Notations and Abbreviations....................................................... 136

Chapter 6 Integrated Temperature-Cycling Acceleration and Test 139

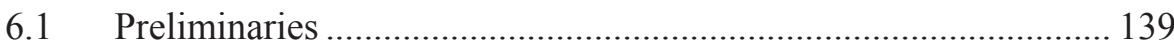

6.1.1 Circuit under Test and Test Access Mechanism ....................... 142

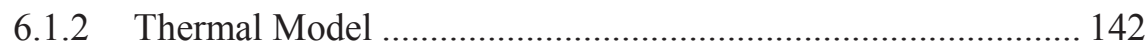


6.1.3 Temperature Cycling Model ..................................................... 144

6.2 Motivational Examples ............................................................... 144

6.2.1 ATC Rate for a Simple Scenario................................................. 144

6.2.2 Optimal Cycling in a Simplified Scenario ................................. 146

6.2.3 Effect of the Test Application Order.......................................... 149

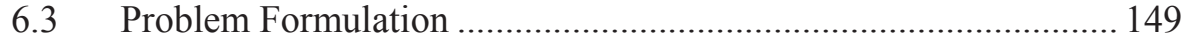

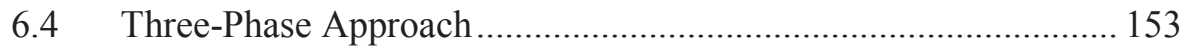

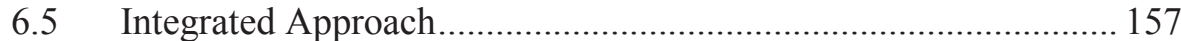

6.5.1 Path-Graph Scheduling Algorithm............................................ 160

6.5.2 Length of the Power Averaging Window .................................. 163

6.5.3 Priorities for TAM Access ..................................................... 164

6.5.4 Node Ordering in the Test Graph............................................. 165

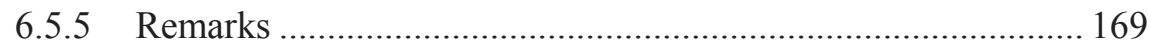

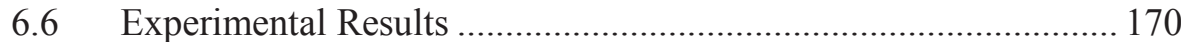

6.6.1 Cycling Acceleration............................................................... 170

6.6.2 Performance of the Integrated Approach .................................. 173

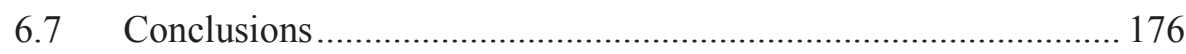

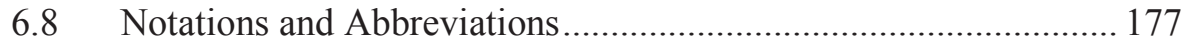

$\begin{array}{ll}\text { Chapter } 7 \text { Conclusions and Future Work } & 181\end{array}$

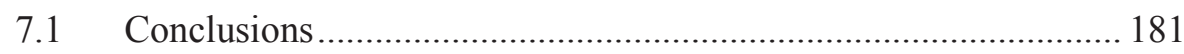

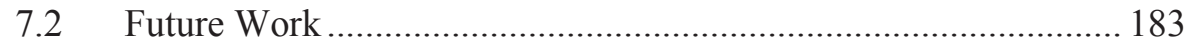

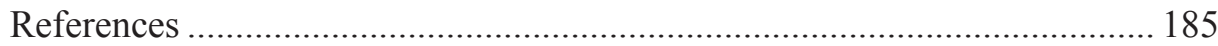




\section{Chapter 1 Introduction}

This thesis deals with temperature-related test issues. We focus on manufacturing test of digital electronics that are produced by Very Large Scale Integration (VLSI) techniques. The thermal test issues that are dealt with in this thesis result in two categories of imperfect products being sent to market: (1) products that are defective and (2) products that even though are fully functional at the beginning, will fail during the field operation shortly after being employed.

The test issues are considered for System-on-Chip (SoC) designs where usually a core-based test architecture is in place. In such cases, the Test Access Mechanism (TAM) is most often scan-based. We focus mainly on advanced SoCs, where a fabrication technique with very small feature size is used, usually referred to as deep submicron technology.

Reducing the feature size has been a mean to integrate more functionality within an Integrated Circuit (IC) with good operational speed, manageable power consumption, and acceptable production cost. This trend cannot be endlessly continued, as the feature size is getting close to the size of a single atom. An alternative for integrating more functionality into a single package is 3D Stacked IC (3D-SIC) technology. 3D-SIC technology can efficiently bond multiple dies into a single package. In this thesis, sometimes we refer to this package as an IC. This thesis focuses on advanced SoCs that have very small feature size or are manufactured by 3D-SIC technology. These technologies are affected by temperaturerelated testing and reliability issues.

This chapter continues with the motivations for this thesis. Then a summary of contributions is given, followed by a list of the author's publications that contain parts of these contributions. Finally, the organization of the thesis is explained. 


\section{Chapter 1}

\subsection{Motivation}

As the feature size is getting smaller, some parts of a modern IC must include a precise small number of certain atoms ${ }^{1}$. Having a few atoms more or less than the planned number will therefore result in a significant change in the characteristics of the circuit. The manufacturing Process Variation (PV) for older technologies that have a relatively large feature size is negligible. However, for an advanced SoC, new techniques are required to address the effects of the PV that is no longer negligible. PV includes variations in the geometry of the chips' components and variation in the properties of the chips' materials. For example, the effective channel length may vary and result in variation of the threshold voltage and subthreshold leakage. These variations will result in differences in several aspects of the circuit's performance including its leakage current which is an important contributor to the overall power consumption. Consequently, the chips will experience power and temperature variations [Choi07, Nebe197].

This means that the thermal aspects of hardware testing must be revised to prevent potential damages. An important thermal issue with testing of advanced SoC has been thermal safety. Advanced SoCs suffer from exceedingly large power densities under test, so much so that the testing must be slowed down to allow for cooling; otherwise the IC under test will overheat. In general, a fast testing procedure is desirable to reduce the testing costs. But in this case, a bit of testing speed is traded off to avoid overheating. Overheating may result in good dies failing the test, since the die's temperature is higher than the intended operational temperatures. Worse than this, is the situation that dies are damaged because their temperatures even exceed the safe temperature limit.

The overheating problem can be efficiently addressed by carefully scheduling the tests. This includes leaving the appropriate amount of cooling intervals in the schedule, just as required. This can be achieved with the help of temperature simulation. An important assumption for existing simulation based techniques is that all the dies have similar

${ }^{1}$ For example see the number of dopant atoms:

http://www.itrs.net/itwg/beyond_cmos/2008ERD_December/02_4_Architectur e SuhwanKim.pdf 
thermal behavior. Therefore, the result of temperature simulations and thus the generated test schedules are valid for all of the dies.

Process variation renders the above assumption untrue for advanced SoCs. What happens with one die is different from another die. One die may work warmer than the other, therefore needing more cooling. Otherwise, it is overheated. On the other hand the die that works colder can be tested faster, saving valuable testing time thus reducing the costs. This means that statistical approaches for temperature and PV-aware test scheduling are required, as introduced in this thesis.

Temperature plays also an important role in testing. For example some of the defects are activated only at high temperatures. This means that the device works perfectly at low temperatures, but fails when it is too hot.

High-temperature defects are very common; therefore many existing techniques stress the die with high temperature while testing. They are common since the resistive opens in metals are common. Some resistive open defects only manifest themselves at high temperatures since the resistivity temperature-coefficient of the involved metals is positive. A large number of interconnects including the crucial clock network are made of metals.

Beside these temperature-dependent defects, there are other defects that depend on temperature. For example, the signal delay depends on the temperature. In an advanced SoC, an extensive clock network runs all over the IC to assure the correct timing of the operations. Some areas in the IC might be hot, while other areas are cold. Exacerbated by negative effects of process variation or otherwise minor defects, this may result in some signal paths being much slower than intended. This can result in timing errors that occur only when certain sites have certain temperatures (usually very different temperatures). This type of defects can only be detected when certain temperatures are enforced on certain sites in the IC. These temperature arrangements can be captured by a temperature map that shows the temperatures for different sites in the IC. Some defects may need their corresponding temperature map to be enforced while testing for them.

A temperature map also implies certain temperature gradients that are temperature differences among different sites. Temperature gradients have an effect on detection of early-life failures. So far we focused on defects that exist immediately after the manufacturing. However, there are defects 


\section{Chapter 1}

that even though do not exist just after the manufacturing, will occur shortly after the device is being used. Burn-in techniques to speed up the device's early life before testing in order to detect certain early-life failures already exist. A burn-in technique is to operate the ICs in a hot environment usually with increased voltage. This speeds up a number of aging mechanisms including the electromigration. Recent research has shown that some early-life failures develop in sites that experience large temperature gradients [Smorodin08]. The defect-related gradients can be captured with a temperature map that is enforced on the IC using the techniques proposed in this thesis.

Another phenomenon that is related to early-life failures is temperature cycling. Exposing the IC to a number of large-scale temperature changes before testing it, makes some early-life failures detectable. A simple burnin will not help to detect these early-life defects and the affected devices will fail shortly after being employed in the field. The existing temperaturecycling tests use temperature chambers [Mil04] and, therefore, the temperature-cycling test is costly. A low-cost temperature-cycling test is proposed in this thesis that uses high-power tests, among other stimuli, to enforce the required amount of cycling on the IC.

\subsection{Contributions}

The first contribution of this thesis is the development of stochastic approaches for thermally-safe and multi-temperature testing under large process variation. The usual cost function for test scheduling is the deterministic test application time which is not appropriate for the situations in which some dies will be overheated due to the negative consequences of process variation. A probabilistic cost function is introduced to include the cost of the overheated ICs. Later on, for multitemperature testing, this cost function is extended to take the cost of the test-escapes (due to temperature-dependent defect) into account. Adaptive approaches, which utilize these cost functions, are proposed to deal with intra-die variations and temperature fluctuations over time [Aghaee11a, Aghaee14b]. Test scheduling techniques that take the temperature into account use a thermal simulator in order to estimate the temperatures before the actual testing. A fast temperature simulation technique is introduced to facilitate faster process-variation aware schedule generation [Aghaee13a]. 
The second contribution of this thesis is a collection of techniques for enforcing the given temperature maps on the ICs. Enforcing certain temperature gradients on an IC for a given time makes the related gradientdependent early-life failures detectable by a targeted test performed later [Aghaee14a]. Enforcing certain temperature maps while testing for gradient-dependent defects (including some hard-to-detect delay faults) helps to detect them [Aghaee13b]. Ordering these temperature maps and consequently their related tests in an effective manner can reduce the test application time, as proposed in [Aghaee15b].

The third and last contribution of this thesis targets cycling-dependent early-life failures. The proposed algorithm utilizes the normal tests (tests not related to cycling) and other stimuli in order to enforce a high level of temperature-cycling activity. This is performed in a controlled manner, so that no overheating or excessive cycling threatens the IC or test performance [Aghaee15a]. The order of the tests affects the dissipated power in the circuit under test. This fact is utilized by the proposed algorithm to achieve a short test application time (including the temperature-cycling time).

\subsection{Publications}

The contributions of this thesis are reported in the following articles:

$\mathrm{N}$ Aghaee, Z He, Z Peng, P Eles. Temperature-aware SoC test scheduling considering inter-chip process variation. 19th IEEE Asian Test Symposium (ATS), pp 395-398. Shanghai, China, Dec 2010.

$\mathrm{N}$ Aghaee, $\mathrm{Z}$ Peng, $\mathrm{P}$ Eles. Adaptive temperature-aware SoC test scheduling considering process variation. 14th Euromicro Conference on Digital System Design (DSD), pp 197-204. Oulu, Finland, Aug 2011.

N Aghaee, Z Peng, P Eles. Process-variation and temperature aware SoC test scheduling using particle swarm optimization. 6th IEEE International Design and Test Workshop (IDT), pp 1-6. Beirut, Lebanon, Dec 2011.

$\mathrm{N}$ Aghaee, Z Peng, P Eles. Process-variation and temperature aware SoC test scheduling technique. Journal of Electronic Testing: Theory and Applications, vol 29, no 4, pp 499-520. Aug 2013. 
N Aghaee, Z Peng, P Eles. Temperature-gradient based test scheduling for $3 D$ stacked ICs. 20th IEEE International Conference on Electronics, Circuits, and Systems (ICECS), pp 405-408. Abu Dhabi, UAE, Dec 2013.

$\mathrm{N}$ Aghaee, Z Peng, P Eles. Process-variation aware multi-temperature test scheduling. 27th International Conference on VLSI Design (VLSID), pp 32-37. Mumbai, India, Jan 2014.

N Aghaee, Z Peng, P Eles. An efficient temperature-gradient based burnin technique for $3 D$ stacked ICs. Design, Automation and Test in Europe Conference (DATE). Dresden, Germany, Mar 2014.

$\mathrm{N}$ Aghaee, Z Peng, P Eles. An integrated temperature-cycling acceleration and test technique for 3D stacked ICs. 20th Asia and South Pacific Design Automation Conference (ASP-DAC), pp 526-531. Chiba, Japan, Jan 2015.

$\mathrm{N}$ Aghaee, Z Peng, P Eles. Temperature-gradient-based burn-in and test scheduling for 3-D stacked ICs. IEEE Transactions on Very Large Scale Integration (VLSI) Systems, Accepted.

$\mathrm{N}$ Aghaee, Z Peng, $\mathrm{P}$ Eles. Efficient test application for rapid multitemperature testing. 25th Great Lakes Symposium on VLSI (GLSVLSI), pp 3-8. Pittsburgh, PA, USA, May 2015.

$\mathrm{N}$ Aghaee, Z Peng, P Eles. A test-ordering based temperature-cycling acceleration techniques for $3 D$ stacked ICs. Journal of Electronic Testing: Theory and Applications, Accepted.

\subsection{Thesis Organization}

This thesis is organized in 7 chapters. The current chapter, chapter 1 , is the introduction. The next chapter, chapter 2, explains the preliminaries. Related work is reviewed in chapter 3. Chapter 4 presents the proposed process-variation aware SoC test scheduling techniques. Chapter 5 focuses on temperature-gradient-based burn-in and test scheduling for 3D-stackedICs. Chapter 6 presents our integrated temperature-cycling acceleration and test techniques. Chapter 7 concludes the thesis and discusses the future work. 


\section{Chapter 2 Preliminaries}

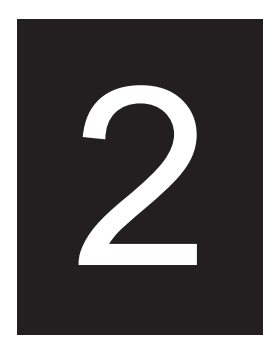

This chapter introduces preliminaries that are helpful for understanding the rest of this thesis. The temperature related defects and tests to detect them are discussed in section 2.1. The testing procedure for core-based systemon-chip designs is explained in section 2.2. The through silicon via and the 3D stacked IC technology that is based on them are briefly introduced in section 2.3. Test scheduling approaches are reviewed in section 2.4. Power and temperature issues are discussed in section 2.5. A temperature simulation technique is introduced in section 2.6. A meta-heuristic approach is introduced in section 2.7.

\subsection{Temperature Related Defects}

A well-known category of manufacturing defects affects the correct operation of the IC just after the manufacturing. Therefore, they can be tested for, immediately after the manufacturing process without any particular environment/temperature-related requirement. We refer to these type of defects as normal defects. Normal defects are relatively easy to detect since they show up just after the manufacturing and can be detected independent of the environmental conditions. An example of such defects is a normal stuck-at fault.

\subsubsection{Temperature Dependent Defects}

Another category of defects is environment-sensitive, and show up only under certain environmental conditions. An important sub-category of these defects are temperature-sensitive defects [Needham98]. For example, some defects show up only when the IC follows a certain temperature pattern [Hagihara97].

An example for such temperature-sensitive defect is a resistive open which is a major cause of test escapes [Needham98]. It occurs when a connection 
between two circuit nodes has a conductance high enough to be considered connected at normal temperatures. But at high temperatures the conductance decreases so much that the connection is considered disconnected. This may occur since usually most of interconnects on the chip are made from metals and the conductance of those metals has negative temperature coefficient. Therefore, it is expected that a large number of such defects appear at high temperatures. On the other hand, we have other defects that manifest themselves differently with respect to temperature. For example, in [Needham98] a defect ("Dark Via") is reported that "had previously passed all production tests, but then failed a monitor test at cold temperature". Several other defects are also identified in [Needham98] that similarly appear only at low temperatures.

Besides the temperature coefficient for conductivity of the material, thermal expansion may also contribute to temperature-dependent defects. The Dark Via defect, which appears at low temperature, could be seen as voids between interconnect and via [Needham98, Segura04]. This observation could be explained with thermal expansion in metals that fills up the voids and increases the conductivity. This effect is illustrated in Figure 2.1.1, where large voids at low temperature shrink at high temperature because of thermal expansion. Therefore, the conductance of the via may increase albeit the reduced conductivity of the via's constructing material.

Other similar defects also exist. For example, some defects for a different technology (i.e., copper-based interconnects) are studied in [Zschech02] and interface voids are mentioned along with sidewall voids and bulk voids (shown also in Figure 2.1.1) as temperature-dependent defects. Moreover, similar to possible temperature-dependent mechanisms for open defects, one may think of temperature dependent mechanisms for short or bridging defects.

Another type of temperature-dependent defect that is hard to detect is silicide open [Tseng00]. Silicide is used to make local interconnects. In its

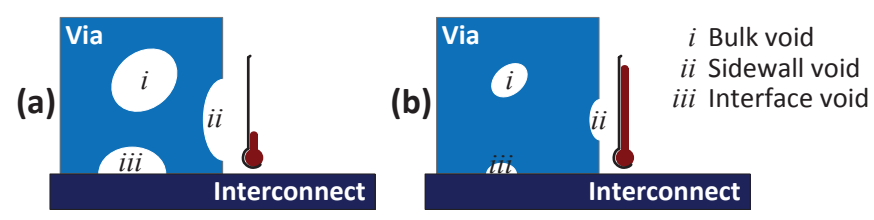

Figure 2.1.1 Voids in a via create a resistive open

(a) Large voids at low temperature. (b) At high temperature, materials expand and voids shrink. 
perfect condition, such a local interconnect has a positive temperature coefficient for resistance, but a defective one will have it as negative. Detecting such defects at normal temperature is difficult since their difference is not recognizable. Testing at low temperatures is a good solution since there will be a recognizable difference between the perfect and the defective interconnects [Tseng00].

Resistive-open and stuck-open defects are experimentally studied in [Li01]. The resistive-opens occur more frequently (39 samples) compared to stuck-open defects (11 samples) [Li01]. By knowing the location of the resistive defects and the materials involved in those defects, the proper test temperatures can be found and the appropriate tests can be developed [Li01].

Interconnect malfunctions (e.g., opens and shorts) are not the only sources of temperature-dependent defects; transistor malfunctions are also a source of concern. This issue is studied in [Long04] and the impact of temperature is demonstrated. The thermal behavior of a transistor depends on its quiescent point and therefore higher or lower temperatures, per se, do not imply better or worst results. Usually, in order to minimize the effect of the temperature, transistors are biased at the Zero-Temperature-Coefficient (ZTC) point. ZTC is a point where the temperature will not affect the transistor behavior. The problem is that there will be variations in the actual quiescent points of the manufactured transistors and therefore temperature will affect them. This will lead to defects that are hard to detect. Multitemperature testing can help to detect such defects [Long04].

\subsubsection{Early Life Failures}

Another category of defects consists of early-life failures. These can be seen as manufacturing imperfections that are not manifesting as a defect just after the manufacturing and therefore cannot be detected by the manufacturing test that is performed immediately after the fabrication. A burn-in process is usually used to push the IC through its early-life in an accelerated manner. The existing techniques operate the device under high temperature and perhaps with increased voltage and/or frequency. These techniques handle the normal early-life failures that can be efficiently accelerated this way. Two subcategories of early-life failures that are different from the usual ones are explained below. 
There are early-life failures that show up at certain sites in the IC where large temperature gradients are in place for relatively long periods of time [Smorodin08]. In order to efficiently detect these defects, corresponding temperature gradients must be enforced for a certain duration of time before testing. The second type of defects are those that are made detectable by temperature cycling. This means that the device goes through an aggressive temperature cycling before being tested for the related defects [Mil04]. This way some other imperfections that are not detectable immediately after the manufacturing can be detected.

\subsubsection{Delay Faults}

Another category of defects that have similar features with some of the temperature related defects mentioned above, consists of delay-related faults. These happen when a signal propagates slower (faster in some cases in relative terms) than expected (e.g., clock signal affected by skew). This may happen due to temperature gradients and usually results in wrong data being latched in memory elements. This can be due to data and clock timings not being correct with respect to each other (e.g., due to different temperatures at different sites). It can, also, be that the IC under test cannot work at the intended frequency, however it can work correctly at a slower clock. At-speed and delay tests are usually used to detect these defects [Ahmed05, Higami13, Ko08].

\subsection{Core-Based SoC Testing}

A simple explanation for testing is that certain stimuli are applied to the site of the targeted defect to activate it and then the circuit outputs are compared against the correct outputs to detect the defect. In order to generate such a test, the circuit model and the possible defect models must be analyzed. This is a tedious task best done with the help of a computer algorithm. Therefore, an Automated Test Pattern Generation (ATPG) tool is used to generate the tests that cover a large number of defects while the tests are kept acceptably short [Abramovici94].

The decision about which defects to target and which tests to include in the test procedure of a certain product has a number of aspects. Incorporating tests for all of the defects, in a modern system-on-chip, will make the test application time very long. Testing costs are considerable, especially if costly test equipment are involved. But shipping defective devices will also cost, since they are usually covered by the manufacturer's guarantee. The 
failures that show up after the device has left the fabrication and test facility will cost much more than the defective device's own cost [Davis94]. The testing process is therefore designed to minimize the overall cost. The other aspect to be considered is reliability for safety-critical applications. The devices manufactured for safety-critical applications usually go through much more elaborate tests to comply with the high reliability requirements.

A modern system-on-chip includes a large number of memory elements (e.g., flip flops and registers) and therefore the number of states that such digital designs include is huge. Moreover, taking the circuit from one state to another state that is needed for some other tests can be very time consuming. This is one of the motivations for Design for Testability (DfT) techniques that include a Test Access Mechanism (TAM) on the corebased system-on-chips.

A test access mechanism is used to provide test access to all the cores. There might be some other testable modules in a system-on-chip that are not conventional cores. These modules are also accessible using the TAM. There is always a trade-off between the test acceleration gained by inclusion of a TAM and the cost of the TAM itself that includes its area on the die, the delays that it adds to the signal paths, and its static power consumption. The TAM design is usually kept small to avoid these overheads. Therefore, it is extremely unlikely to be able to provide simultaneous access to all modules. Consequently, during the test some of the modules must wait while other modules are being tested.

The tests are usually performed using Automated Test Equipment (ATE) which put the device in the test mode, feed it with stimuli, and check the circuit under tests' outputs for defects.

\subsection{D Stacked IC Testing}

Existing systems-on-chip like Apple A8X and Xbox One have 3 and 5 billion (i.e., $\times 10^{9}$ ) transistors, respectively. Larger number of transistors have already been integrated. For example Intel Xeon E5-2600 v3 has 5.6 billion transistors [Intel13], Nvidia Kepler GK110 has 7.1 billion transistors [Nvidia12] and Xilinx Virtex UltraScale XCVU440 has 20 billion [Santarini14]. These indicate the extremely large number of transistors that will be integrated into advanced system-on-chips in order to provide a wider range of functionalities as well as higher computational power. 
More functions as well as higher computational power are traditionally achieved by shrinking the feature size as well as some other minor improvements so that a large number of possibly faster transistors fit on a single die. For more than that, a number of dies must be connected. These inter-die interconnects are usually long and thick. Moreover, a relatively small number of interconnects can be made per die area (i.e., low interconnect density). These lead to high power consumption as well as low data transmission rate.

A promising technology for efficiently connecting different dies is based on Through Silicon Vias (TSV). A through silicon via is a via that runs throughout the bulk silicon and allows the dies to be stacked on top of each other while making electrical connections. The ICs fabricated this way are called 3D Stacked ICs (3D-SIC). This technology supports high density signal connections with a short wire length that translates into high bandwidth communication (both number of lines and the frequency that they support) with a small power consumption.

TSVs are manufactured in the individual dies. They are initially contained within the die, since their length is smaller than the die's thickness. Therefore, a thinning step follows in order to carefully remove extra thickness of the die. After the thinning process, the TSVs reach the surface of the die.

On the surface of the die the so called micro-bumps are placed. The microbumps are places where electrical connections, for example by soldering, are made. The dies must be carefully aligned and then correct bonding can take place.

The steps in the manufacturing process may involve multiple bonding stages. A testing procedure at each of these stages may help to reduce the overall costs. These tests are referred to as pre-bond, mid-bond, and postbond test stages. The pre-bond test is performed before bonding when the die is separate. If a defect goes undetected to the next steps, some other potentially perfect dies as well as the bonding efforts are wasted because of the defective die. Similarly, a mid-bond test may be helpful especially if an expensive die is going to be bonded to a low-cost partial stack. In this case, it might be a good idea to test the partial stack before bonding. At the end of the bonding process, a post-bond test can be performed. 
The bonding can be, also, done with wafers instead of the individual dies. In this case, the wafers are aligned and bonded and then diced. Since the dies are still not diced during the bonding, it is not possible to choose the non-defective dies to be bonded together. In such a scenario, the wafers can be matched, positioned, and aligned so that the low defect-rate areas of the two wafers meet each other. The probability of ending up with defective stacks are reduced this way, although it is not possible to fully prevent good dies being wasted.

So far we explained die to die bonding and then wafer to wafer bonding. Another alternative for bonding is die to wafer bonding. In this case, a particular layer in the 3D-SIC structure is diced into dies while the other wafer is not diced. This way bonding known bad dies can be avoided.

The TSV manufacturing process and bonding process are new sources of defect that do not exist for normal 2D ICs. Therefore, a more elaborate testing process may be required, especially for defects that are related to the TSV fabrication or the bonding process.

For 3D stacked IC testing, the TAM is designed so that the test access is possible at different test stages [Ieee14a]. 3D-SICs experience more thermal issues than the conventional 2D ICs. These include the issues that affect the conventional 2D ICs as well as thermo-mechanical issues related to TSV technology. Moreover, the dies cannot cool as efficiently as 2D ICs that usually have many low-resistance thermal paths for cooling. The situation is particularly difficult for dies located in the middle of the stack.

\subsection{Test Scheduling}

As mentioned before, the test access mechanism, in either 2D or 3D SoCs, is a resource bottleneck for testing. Therefore, tests must be scheduled in order to minimize the test application time. A test schedule determines at each time-point which modules must run their tests. Moreover, it determines which test must be performed for the module.

Test scheduling can be done with or without partitioning and interleaving. Schedules without partitioning [Chou97, Zorian93] are simpler but in general result in large test application times. In this case, when a module starts a certain test it runs to the test's completion and the schedule cannot make changes when a test is being applied. Nowadays, partitioning and interleaving of tests is common [Marinissen00]. In this case, a test can be 
halted for a while and other modules may use the released TAM resources. This thesis uses test partitioning and interleaving for all the proposed scheduling approaches.

The authors in [Iyengar02] have formulated the test scheduling problem as a rectangle packing problem. The problem is proven to be NP-complete and is solved using a Mixed-Integer Linear Programming (MILP) approach in [Chakrabarty00]. The test scheduling problem becomes even more complicated when, for instance, the thermal issues must be taken into account.

Here we briefly explain the main ideas related to test scheduling using an example. Assume that the SoC under test consists of three modules $m_{0}$, $m_{1}$, and $m_{2}$ as shown in Figure 2.4.1a. Assume that the test access mechanism can accommodate only two of these modules at a time $(W=$ 2 , where TAM width is denoted by $W$ ).

There are two Built-In Self-Test (BIST) modules $b_{0}$ and $b_{1}$ as shown in Figure 2.4.1a. Each of them performs only a part of the tests for the corresponding module. $b_{0}$ uses the TAM to test $m_{0}$ but $b_{1}$ is directly connected to $m_{1}$ and can test it without occupying the TAM. Assume that each module has four tests and that each one of them is a node in a directed path-graph (i.e., there is only one path in the test graph). The $k^{\text {th }}$ test for module $m$ is denoted by $n_{m, k-1}$ as shown in Figure 2.4.1b. The forth test for module $m_{0}$ (i.e., $n_{0,3}$ ) is performed by the $b_{0}$ BIST while $n_{1,3}$ is performed by $b_{1}$. The rest of the tests (marked as normal in Figure 2.4.1b) are performed using an ATE through TAM.

Since the TAM cannot support simultaneous testing of all modules, the tests must be scheduled. A shorter test application time is desirable and therefore the test schedule must be optimized for a minimal test applications time. In general, there could be other constraints, in addition to TAM, including power, temperature, and tester memory constraints. The

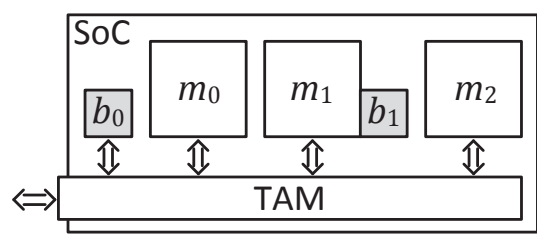

(a)

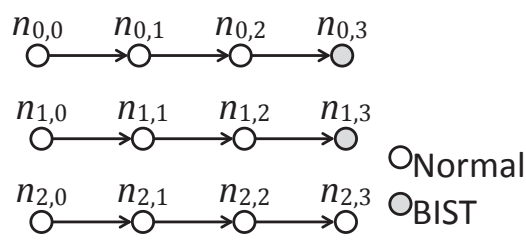

(b)

Figure 2.4.1 Examples for (a) a SoC, and (b) tests 
scheduling objective may include other factors, in addition to test application time, including test throughput and perhaps test coverage considering defect probabilities.

Let us focus only on test application time reduction under TAM limitation. A module can be only in one of these two states: active (i.e., testing) or inactive. A schedule indicates the test cycles (time) that a change in one or more of the modules' states must happen and what that change is. The schedule indicates that at cycle $i_{0}$ modules $m_{0}$ and $m_{1}$ start testing, as indicated in in Figure 2.4.2a. Since the tests' path-graphs are given, there is no need to include the test nodes in the schedule, however, the tests being applied are shown in Figure 2.4.2d. The active modules go through their $1^{\text {st }}, 2^{\text {nd }}$, and $3^{\text {rd }}$ test without any new entry in the schedule.

At test cycle $i_{1}$, the BIST tests $\left(n_{0,3}\right.$ and $\left.n_{1,3}\right)$ start as indicated in Figure 2.4.2c. Since $b_{1}$ has dedicated access to module $m_{1}$, it does not occupy the TAM and, therefore, module $m_{2}$ can gain access to the TAM, as shown in Figure 2.4.2b. Consequently, all three modules are active simultaneously. At test cycle $i_{2}$, testing of $m_{0}$ and $m_{1}$ is complete. Testing of $m_{2}$ continues to completion at cycle $i_{3}$.

In the above example, we assumed that the order of the tests is fixed, but in reality it might be possible to reorder tests to achieve better results. In that case, the nodes (e.g., Figure 2.4.2d) must be included in the schedule. This means that at least two additional entries in the schedule table (Figure 2.4.2a) between cycles $i_{0}$ and $i_{1}$ as well as two more additional entries between cycles $i_{2}$ and $i_{3}$ must be added to indicate transition to new test nodes. (In fact one entry is sufficient since the last node is trivial.)

(a)

\begin{tabular}{|c|c|c|c|c|c|c|}
\hline \multirow{4}{*}{ schedule } & \multicolumn{2}{|c|}{ cycle } & \multirow{2}{*}{$i_{0}$} & & $i_{2}$ & \\
\hline & & $m_{0}$ & & & - & \\
\hline & state & $m_{1}$ & $\sim$ & $\sim$ & - & - \\
\hline & & $m_{2}$ & - & $\sim$ & $\sim$ & - \\
\hline
\end{tabular}

(b)

TAM $\begin{array}{lllllll}m_{1} & m_{1} & m_{1} & m_{2} & m_{2} & m_{2} & m_{2}\end{array}$

(c)

BIST

(d) test node

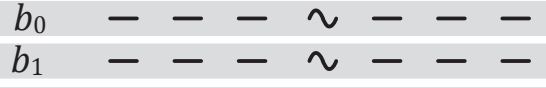

$$
\begin{aligned}
& \begin{array}{llll}
n_{0,0} & n_{0,1} & n_{0,2} & n_{0,3}
\end{array} \\
& \begin{array}{llll}
n_{1,0} & n_{1,1} & n_{1,2} & n_{1,3}
\end{array} \\
& \begin{array}{llll}
n_{2,0} & n_{2,1} & n_{2,2} & n_{2,3}
\end{array}
\end{aligned}
$$

$\sim$ Active

- Inactive

Figure 2.4.2 Example for a test schedule

(a) the test schedule; (b) TAM occupation; (c) BIST activity; (d) test nodes 
Moreover, we assumed that testing is always done for all of the specified tests, but in reality testing may be terminated as soon as a defect is found. In this case the optimization objective (e.g., test application time) is a stochastic quantity (e.g., expected test application time) that is evaluated based on the defect probabilities (or statistics).

A test schedule can be adaptive, depending on certain run-time parameters. An adaptive schedule acts based on the actual value of an otherwise stochastic quantity during the test. An example is sensing the actual temperature and changing the schedule accordingly. In this case, a number of schedule pieces are generated and during the test, the temperature is sensed when required and the schedule-piece that fits the situation is selected.

\subsection{Test Power and Temperature}

The circuit under test consumes power as a result of switching activity during the test process, similar to when the circuit is in operation. In general, power density for digital circuits is increasing by the advancement of technology and increased integration. One of the problems is that this dense power dissipation leads to very high temperatures and can affect the correct system behavior. The situation is worst during the testing. In particular scan-chain based DfT features result in even higher power densities. It is reported that the test power dissipation can be as large as twice the normal power [Bonhomme02, Zorian93].

In order to prevent incorrect device behavior or damage to the device because of high temperature (overheating) something must be done. A category of efficient approaches that do not make the testing unnecessarily long are based on changing the test schedules [Rosinger06]. In order to prevent overheating during the test, temperature simulations are performed before the actual test during the scheduling process. The simulated temperature shows the time intervals in the schedule where overheating may occur. One of the options is to halt the test to allow for cooling at such time intervals. This way, cooling which slows down the testing process is just added to the schedule exactly when it is needed.

Process variation results in large variations in the dissipated power in advanced SoC designs [Cheng00]. This results in considerable variations in the temperature of the device and poses difficulties for the offline temperature-aware test scheduling techniques that are deterministic (e.g., 
[Rosinger06]). To handle this situation, stochastic approaches are proposed in this thesis in chapter 4.

The dissipated power in a circuit depends on the current input values and the circuit's state. The state depends on the previous inputs. Therefore, the dissipated power during the test depends on the tests order [Girard97]. This phenomenon is used in chapter 6 to harvest different power values from the same set of tests.

The power dissipations are calculated based on the given switching activities and the IC power-related characteristics. The actual dissipated power also depends on the leakage current (i.e., static power). The leakage current, itself, depends on the temperature. As mentioned before, always in this thesis a temperature simulation is performed. The simulated temperatures are used to guide the schedule generation. Also, they are used to approximate the static power, the component that depends on the temperature.

Leakage current plays an essential role in thermal run away. Thermal run away is a situation in which the static power, per se, can keep increasing the temperature, even beyond the safe limit. This means that introducing a halt that takes away the dynamic power will not stop the temperature from increasing. Consequently the temperature further increases, increasing the static power and the increased static power increases the temperature, in return [Vassighi06].

This positive feedback loop goes on and on until the circuit is disconnected from the power source or until the circuit is damaged. Once started, this usually goes fast. However, it only starts at high temperatures. In the usual DfT architectures only the dynamic power can be controlled by the schedule. Therefore, in schedule-based solutions, such high temperatures must be avoided.

\subsection{Temperature Simulation}

As mentioned above, in order to estimate the actual temperatures during the test, temperature simulations are performed during the scheduling process. This paradigm has been used in all chapters of this thesis. A temperature simulator consists of a thermal model and an algorithm to analyze it. The thermal model describes the mathematical relation between the IC characteristics, the dissipated power, and the temperatures. 
There exists a range of thermal models. Some of them may focus on the steady state temperatures which means that the dynamic response cannot be obtained. Some other thermal models, only focus on each individual module and ignore the heat transfer among modules. In this thesis we use a thermal model that supports dynamic response analysis and takes the heat transfer among modules into account, similar to the widely used thermal simulator, HotSpot [Huang07, Huang06, Stan03].

This model is a lumped element model meaning that the chip is modeled as a combination of thermal resistances and thermal capacitances. An example for such a thermal model is given in Figure 2.6.1. A typical thermal model consists of a number of lumped elements connected to each other. A connection point of thermal elements is called a node.

An equivalent view is that an IC is divided into small elements each of which is characterized by a single temperature. Each of these small elements is represented as an individual node in the model. In Figure 2.6.1, two cores are modeled as two nodes (i.e., elements) which are connected to two exclusive power sources. Power sources represent the power dissipated by the cores.

Assume that the thermal model consists of $W$ nodes and $C$ is the number of cores. In a high quality thermal model, usually the number of nodes is larger than the number of cores, $C \leq W$, (e.g., six thermal nodes for two cores) as shown in Figure 2.6.1. Assume that $\boldsymbol{P}$ is the power vector and $\boldsymbol{\Theta}$ is the temperature vector. The mathematical representation of the thermal model is a system of ordinary differential equations:

$$
\boldsymbol{A} \times \frac{d}{d t} \boldsymbol{\Theta}+\boldsymbol{B} \times \boldsymbol{\Theta}=\boldsymbol{P} .
$$
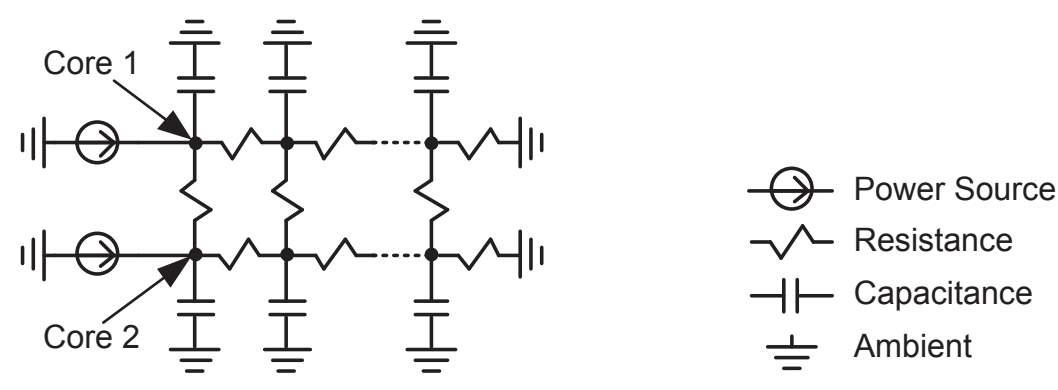

Figure 2.6.1 An example of a lumped element thermal model 
The properties of the thermal model are encapsulated into two $W \times W$ matrices $\boldsymbol{A}$ and $\boldsymbol{B} . \boldsymbol{\Theta}$ and $\boldsymbol{P}$ are $W \times 1$ temperature and power vectors. The mathematical representation of this commonly used model (equation 2.6.1) is a system of linear constant-coefficient differential equations. As an example, assume that a SoC has two cores $(C=2)$ and assume that the model has four nodes $(W=4)$. The expanded characteristic equation of the model is

$$
\left[\begin{array}{cccc}
a_{0} & 0 & 0 & 0 \\
0 & a_{1} & 0 & 0 \\
0 & 0 & a_{2} & 0 \\
0 & 0 & 0 & a_{3}
\end{array}\right] \times \frac{d}{d t}\left[\begin{array}{l}
\theta_{0} \\
\theta_{1} \\
\theta_{2} \\
\theta_{3}
\end{array}\right]+\left[\begin{array}{cccc}
b_{0,0} & b_{0,1} & b_{0,2} & b_{0,3} \\
b_{0,1} & b_{1,1} & b_{1,2} & b_{1,3} \\
b_{0,2} & b_{1,2} & b_{2,2} & b_{2,3} \\
b_{0,3} & b_{1,3} & b_{2,3} & b_{3,3}
\end{array}\right] \times\left[\begin{array}{c}
\theta_{0} \\
\theta_{1} \\
\theta_{2} \\
\theta_{3}
\end{array}\right]=\left[\begin{array}{c}
P_{0} \\
P_{1} \\
0 \\
0
\end{array}\right] .
$$

$\theta_{0}$ and $\theta_{1}$ are core temperatures which should be taken care of. $P_{0}$ and $P_{1}$ are the power values applied to the cores.

For architectural design purposes, usually the dissipated power is assumed to correspond to a fixed scenario. The inputs are the IC characteristics that are varied to find a good design. The outputs are the temperatures that somehow affect the cost function for the architectural design. This viewpoint is useful for example for designing the $\mathrm{TAM}^{1}$. For this view point numerical approximation is a good choice to solve equation 2.6.1.

In order to numerically analyze and solve the combination of the thermal model and the dissipated power values, a time interval which is called a simulation cycle is defined. The length of simulation cycle is determined based on a number of factors including the required accuracy. The computed temperatures are recorded and reported for each simulation cycle. It is common to assume that the power ( $\boldsymbol{P}$ in equation 2.6.1) is constant during a simulation cycle.

The numerical approximations are usually done with very small intermediate steps, and as a result, the complete temperature curve for the interval is meticulously constructed. HotSpot uses the Runge-Kutta method for the numerical approximation [Huang06]. Though only the temperature at the end of the simulation cycle is registered, many points of the temperature curve are calculated.

\footnotetext{
${ }^{1}$ Not the viewpoint of this thesis. In this thesis we assume that the TAM is already designed and given along with the other IC specifications.
} 
This thesis' viewpoint is that the IC characteristics are fixed. The inputs that are varied are the power values. They vary because they depend on the tests and the schedules. A range of different schedules are explored to find a near optimal schedule. The outputs are temperatures. The thermal models work equally well for both of the above viewpoints, whether the IC characteristics are fixed or not. However, the difference in these viewpoints means that different approaches may be appropriate for solving the thermal model.

Since the physical design of the devices is assumed to be fixed, a superposition-based approach as the one suggested in [Yao09] can be used. This superposition-based approach is particularly helpful if the tests are partitioned in advance (before the scheduling process) and if large errors in static power (due to temperature-dependent leakage) are acceptable. In this thesis a third approach different from the Runge-Kutta and the superposition-based approach is used. A fast temperature simulation scheme is proposed in section 4.6.

\subsection{Meta-Heuristic}

The test scheduling process is usually based on a number of decision variables. These decision variables go through an optimization process in order to generate a near optimal test schedule. A cost function is defined to evaluate the quality of alternative schedules which are themselves based on the combinations of the decision variable values. A motivational example explains these concepts. Then, particle swarm optimization, which is a meta-heuristic frequently used in this thesis, is introduced.

\subsubsection{Motivational Example}

A thermal-safe scheduling paradigm is discussed here to explain basic ideas of thermal-aware test scheduling and optimization. The objective is to generate a test schedule with the minimal Test Application Time (TAT). The constraint is that the temperature must not exceed the overheating level denoted by $\theta^{\text {overheating }}$ (this includes a safety margin).

We consider an IC made of only one module. Therefore, there are no constraints for access to modules using the test access mechanism. Assume that the tests dissipate a constant power (including both dynamic and static power) denoted by $P^{T}$. It is assumed that $P^{T}$ is so large that it results in overheating. Usually leakage and clock networks power result in a non- 
zero power dissipation during cooling. This cooling power which is denoted by $P^{C}\left(P^{C}<P^{T}\right)$ results in a rest temperature (denoted by $\theta^{\text {rest }}$ ) that is higher than ambient $\left(\theta^{\text {ambient }} \leq \theta^{\text {rest }}<\theta^{\text {overheating }}\right)$.

The module temperature is initially equal to the ambient temperature denoted by $\theta^{\text {ambient }}$. As discussed above, the test is paused as soon as the temperature reaches $\theta^{\text {overheating }}$. Testing is resumed after sufficient cooling. The question is how much cooling is sufficient. Certain temperature level can be considered as sufficient. Let us denote this sufficient temperature level by $\theta^{s}\left(\theta^{\text {rest }} \leq \theta^{s}<\theta^{\text {overheating }}\right)$. Thus, sufficient-cooling temperature, $\theta^{s}$, is the decision variable in this problem formulation. The temperature curve is plotted in Figure 2.7.1.

Since the power values (i.e., $P^{T}$ and $P^{C}$ ) are constants, the testing and cooling patterns are periodic, as can be seen in Figure 2.7.1. In each of these periods, the testing time is denoted by $t^{T}$ and the cooling time with $t^{C}$. There is, also, a delay associated with starting or resumption of the testing process, denoted by $t^{d}$. This delay is associated with testing equipment and architecture and cannot be changed. A part of this delay, denoted by $t^{d c}$, results in the temperatures to further reduce to a low temperature level, denoted by $\theta^{L}$.

The other part of the switching delay, denoted by $t^{d t}$, results in a shorter effective test time than the testing times, $t^{T}$. Therefore, the actual times when testing takes place is equal to $t^{T}-t^{d t}$. Assuming that one test unit (e.g., a thousand test bits) is applied per second, and assuming that the test length is $T L$ test units, the total number of testing/cooling periods, approximately, is:

$$
N \cong T L /\left(t^{T}-t^{d t}\right)
$$

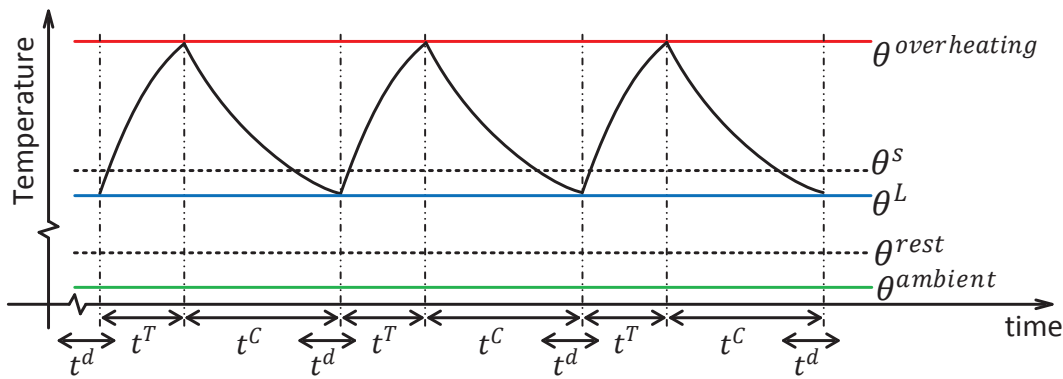

Figure 2.7.1 Temperature curve for a simple thermal-aware testing scenario 
Therefore,

$$
T A T \cong N \times\left(t^{T}+t^{C}\right)=\frac{T L \times\left(t^{T}+t^{C}\right)}{t^{T}-t^{d t}}
$$

Assume that the module under test is thermally modeled by a single thermal element using equation 2.6.1. The module's heat capacitance is denoted by $C$ (analogous to $\boldsymbol{A}$ ). The heat resistance between the module and the ambient is equal to $R$ (analogous to $\boldsymbol{B}^{-1}$ ). In this case, equation 2.6.1 can be described for the testing part of the period as:

$$
\theta^{\text {overheating }}=\theta^{L} \times \exp \left(-\frac{t^{T}}{R \times C}\right)+P^{T} \times R \times\left(1-\exp \left(-\frac{t^{T}}{R \times C}\right)\right)
$$

For the cooling part of the period, the thermal equation can be written as:

$$
\theta^{L}=\theta^{\text {overheating }} \times \exp \left(-\frac{t^{C}}{R \times C}\right)+P^{C} \times R \times\left(1-\exp \left(-\frac{t^{C}}{R \times C}\right)\right)
$$

These equations can be used to compute the values of $t^{T}$ and $t^{C}$ as:

$$
t^{T}=-R C \times \ln \left(\frac{\theta^{\text {overheating }}-P^{T} \times R}{\theta^{L}-P^{T} \times R}\right)
$$

and

$$
t^{C}=-R C \times \ln \left(\frac{\theta^{L}-P^{C} \times R}{\theta^{\text {overheating }}-P^{C} \times R}\right)
$$

Using equations 2.7.1-2, TAT values are plotted for a range of $\theta^{S}$ values in Figure 2.7.2. It is assumed that $\theta^{\text {overheating }}=120^{\circ} \mathrm{C}, P^{T} \times R=$ $150^{\circ} \mathrm{C}$, and $P^{C} \times R=40^{\circ} \mathrm{C}$ (this is the rest temperature, $\theta^{\text {rest }} \cong 40^{\circ} \mathrm{C}$ ). The TAT is minimal when $\theta^{s}=89^{\circ} \mathrm{C}$.

In the above example, there was only one decision variable, no TAM congestion, constant testing and cooling power values, and a simple thermal model. Therefore, the optimization problem was solvable by plotting TAT versus $\theta^{s}$. The problem is that none of the above assumptions are realistic.

In reality there are a number of decision variables (e.g., one $\theta^{s}$ for each module). Because of TAM congestion, a module cannot start/resume testing disregarding of other modules. Testing and cooling powers can be different for different test stimuli and they, also, depend on the temperature. A module's temperature may need to be modeled with several thermal elements. A thermal element's temperature depends on the test 


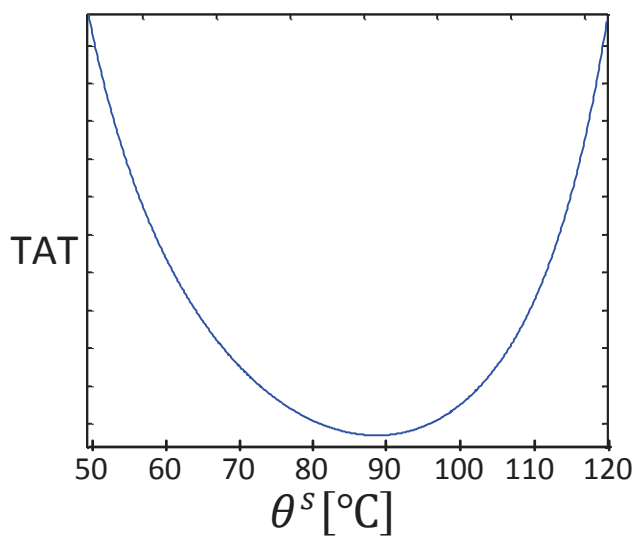

Figure 2.7.2 Test application time versus sufficient-cooling temperature

stimuli power and the temperature of the adjacent thermal elements. This situation is much more complex than the above example and it will be extremely time-consuming to find the exact optimal schedule. Therefore, a near-optimal solution that can be found in an affordably short time is preferred. For this purpose, particle swarm optimization which is a population-based meta-heuristic is used in this thesis.

\subsubsection{Particle Swarm Optimization}

Let us review a more realistic version of the thermal-safe scheduling discussed in the previous section. For this purpose the IC's temperature must be simulated offline during the schedule generation, as shown in Figure 2.7.3. As soon as the temperature reaches the overheating level denoted by $\theta^{\text {overheating }}$ the test is halted to allow for cooling. For example at test cycle $i_{1}$ testing is paused (module is inactive) to allow for cooling. This is registered in the schedule table as shown in Figure 2.7.3b-c.

Temperature simulation continues and when the temperature reduces to $\theta^{s}$ (sufficient-cooling temperature), the module activity (i.e., testing) may resume. The actual resumption may be delayed due to testing equipment and architecture characteristics. Moreover, the delay may be due to TAM congestion which forces the module to wait for test access. In this example, testing resumes at test cycle $i_{2}$, as registered in the schedule table in Figure 2.7.3b-c. Since the power values are not constant, the heating time between $i_{2}$ and $i_{3}$ is shorter than the heating time between $i_{4}$ and $i_{5}$. 


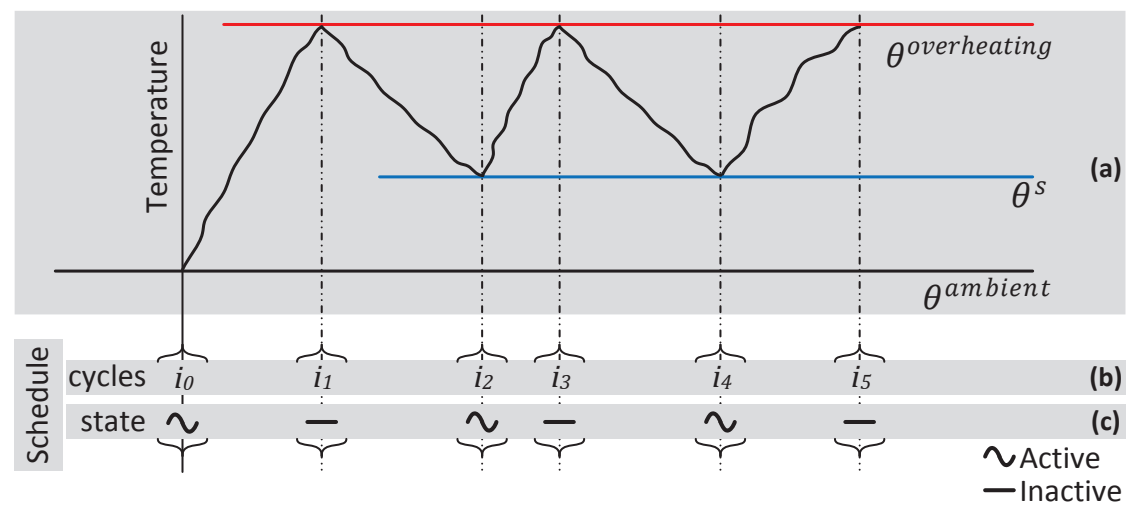

Figure 2.7.3 Test scheduling based on temperature simulation

(a) Temperature curve; (b) test cycles registered in the schedule table; (c) module states in the schedule table. (Curves are only illustrative.)

This constructive, on-the-fly, and temperature-simulation-based scheduling continues until all the tests are scheduled. This point marks the test application time that must be minimized using a meta-heuristic ${ }^{2}$.

There are a number of meta-heuristics that can be used for optimization. A population-based meta-heuristic is usually used in such situations. A wellknown example for such category of algorithms is the genetic algorithm [Falkenauer98, Maulik00]. In this thesis we often use a Particle Swarm Optimization (PSO) technique. Here we briefly explain the PSO which is used in this thesis.

Particle swarm optimization mimics the social behavior of a swarm searching for food [Poli07]. Each individual member of the swarm is called a particle. A particle is represented by two attributes, its location and its velocity. The location in fact is a solution which, usually, is represented by a coordinate in a Cartesian system. The velocity keeps the particles moving in the search space.

Each particle remembers its previous best location, and in addition to this individual memory, the swarm remembers the best location any of its particles have visited before, the global best. The previous bests and the global best are then used to give a hint to the random velocities. A

2 The technique used in this example is from [He08a]. The actual optimization problems in this thesis are more sophisticated than this example. 
canonical form of the particle swarm optimization is expressed by the following equations [Poli07]:

$$
\begin{aligned}
& \text { velocity }^{\text {new }}=0.7298 \times \text { \{velocity }^{\text {previous }}+ \\
& +\left[2.05 \times \text { random }_{0} \times\left(\text { previousBest }- \text { location }^{\text {previous }}\right)\right]+ \\
& +\left[2.05 \times \text { random }_{1} \times\left(\text { globalBest }^{\left.\left.\left.- \text {location }^{\text {previous }}\right)\right]\right\}}\right.\right.
\end{aligned}
$$

location $^{\text {new }}=$ location $^{\text {previous }}+$ velocity $^{\text {new }}$

This canonical form of the particle swarm optimization uses equation 2.7.3 to update the velocity. The coefficients in equation $2.7 .3(0.7298,2.05$, and 2.05) are given as a part of the chosen canonical form. The random 0 and random $_{1}$ are two distinct random numbers between 0 and 1 which are renewed iteratively. The location and velocity on the right hand side of equation 2.7.3 are the previous values and the left hand side velocity is the new value. The new location is the sum of the previous location and the new velocity as expressed in equation 2.7.4. Sometimes an action is needed to prevent the new location from going outside the valid search space. This can be done by limiting its value (e.g., by changing its value) to the valid extremes.

For example, in the above example the decision variable (i.e., sufficientcooling temperature) must be larger than the rest temperature and smaller than the overheating temperature $\left(\theta^{\text {rest }}<\theta^{s}<\theta^{\text {overheating }}\right)$. Smaller values will result in an infinite loop in the scheduling algorithm since the temperature will never become smaller than $\theta^{\text {rest }}$. Larger values have a similar effect, since when cooling the temperature only decreases and cannot increase beyond $\theta^{\text {overheating }}$. In these cases the scheduling algorithm will wait forever for a temperature that cannot be reached.

A simple form of the particle swarm optimization is presented below:

1. Generate the initial locations (in the valid search space)

2. Generate random initial velocities (in a reasonable range)

3. Evaluate the solutions

4. Find the best solutions as follows:

a. Loop for all particles.

i. If the current location is better than the previous best location replace it and check if it is better than the global best, if so, replace the global best. (For the first iteration, copy the current solution as previous best, and find the global best among the previous best solutions.)

5. If the termination condition is met, exit with the global best as final solution.

6. Update the Swarm as follows:

a. Loop for particles:

i. $\quad$ Update the velocities according to equation 2.7.3

ii. Update the particle's location according to equation 2.7.4 
iii. Limit the location to the valid extent of search space

7. GO TO point 3 .

In order to see how PSO works, assume that the lower location value corresponds to more cooling (e.g., $\theta^{s}$ as the decision variable in Figure 2.7.3a). Therefore, a negative velocity guides the particle towards more cooling and if more cooling in this iteration helps to reduce the cost (TAT for the above example), it is reasonable to keep a negative velocity for the next iteration as well.

If such a move makes the particle the best in the swarm, that will affect the velocities of other particles as well. If a particle is at great distance to the promising search region, its velocity will, generally, be larger due to large difference values in equation 2.7.3. This allows fast move towards a better area. The particle slows down when it approaches the promising area due to small difference values in equation 2.7.3. This enables a detailed search in the promising areas.

The evaluation of the cost function (e.g., schedule length) for different particles can be performed in parallel (e.g., using multiple threads). This might be very helpful, especially if temperature simulations are involved. In many cases (e.g., scheduling) as the evaluation proceeds, the cost (e.g., test application time) grows. Therefore, at any time-point for a particle (parallel thread) it becomes certain that it has no chance of affecting the local best (and, therefore, the global best) the thread can be stopped. There is no need to further evaluate the particle, since it is not going to be used in equation 2.7.3.

This is important since the CPU time is usually proportional to the schedule length and, hence, to the test application time. Bad schedules that do not contribute to equation 2.7.3 usually correspond to a long test application time and take a long CPU time to complete. Therefore, aborting their corresponding threads drastically speeds up the search. As soon as a good particle is found, the bad ones can be stopped. 


\section{Chapter 3 Related Work}

Recently thermal issues affecting testing procedures have been intensively studied [Tadayon00]. A promising class of solutions are scheduling-based [He06b, Rosinger06]. Some of these issues, like thermal-safe testing, have been previously addressed and some of them like issues related to the process variation, temperature gradients, and temperature cycling have not been sufficiently studied. This chapter provides an overview of the related work.

\subsection{SoC Test Scheduling}

An optimal solution for the test scheduling problem for core-based systems is presented in [Chakrabarty00, Chakrabarty02]. Test data and the test access mechanism are assumed to be given. The decision variables are the start times for the tests. The optimization objective is to minimize the total test application time. It is shown that this problem is NP-complete. A solution based on a mixed-integer linear programming (MILP) formulation is suggested. It is shown that MILP solution is too slow for large designs. Consequently, an efficient heuristic algorithm for dealing with such large designs is presented in [Chakrabarty00, Chakrabarty02].

A method to address both the scheduling and the design of DfT features, together, is proposed in [Huang01, Huang02]. The objective is to reduce the test application time and the constraints include the power budget. The problem is formulated as a two-dimensional bin-packing problem which is solved using a best-fit heuristic algorithm [Huang01, Huang02].

A test scheduling approach that supports test preemption is introduced in [Iyengar01]. The constraints include the power budget and precedence constraints. Precedence constraints impose that the generated schedules preserve desirable orderings among tests. Allowing test preemptions results into shorter schedules [Iyengar01]. 
A test design approach that optimizes some of the DfT features along with the schedule generation is proposed in [Zou03]. A simulated annealing based heuristic is used to solve the test scheduling problem that is formulated as a two-dimensional bin packing problem. The width of the core wrapper is one of the decision variables optimized by the simulated annealing algorithm [Zou03].

An abort on first fail approach with test power constraint is introduced in [He06a]. Abort on first fail means that the test is terminated as soon as a defect is detected. Defect probabilities for cores and power constraints are assumed as given. Test partitioning is performed by the scheduling algorithm. A heuristic generates the test schedules with partitioning, aiming at a minimal test application time [He06a].

A test scheduling approach for 3D-SIC is proposed in [SenGupta12]. For normal 2D ICs, the same test schedule is used both at wafer sort and at package test. In a 3D-SIC a number of dies are integrated into a single package. Therefore, the package will have a collection of the tests for individual dies in addition to tests for the TSV interconnects. A technique for co-optimization of the wafer sort and the package test is proposed for 3D-SIC. The proposed approach utilizes an on-chip JTAG infrastructure and efficiently re-uses JTAG lines to perform testing of different cores [SenGupta12].

\subsection{D Stacked IC Testing}

Miniaturization and performance requirements result in the usage of new technologies, such as 3D-SICs based on TSVs. Their advanced fabrication processes as well as physical access limitations result in major testing challenges. The manufacturing steps of TSV-based ICs and their testing challenges are introduced in [Marinissen09]. The necessary steps for wafer-level and package-level testing in addition to the required test data, wafer-level probe access, and DfT features are discussed [Marinissen09].

A technique for clock network synthesis that supports pre-bond testability for 3D-SICs is proposed in [Kim10]. This prevents bonding of a bad die to good dies by testing the dies before stacking. The pre-bond clock network testing requires a complete $2 \mathrm{D}$ clock tree on each die. The proposed tree topology generation algorithm uses a minimal number of TSV-related buffer resources. Moreover, self-controlled clock transmission gates are proposed in order to eliminate transmission gate control lines. 
Consequently, the number of TSVs and clock-network power consumption are reduced [Kim10].

A DfT architecture for 3D-SICs allowing pre-bond die testing as well as mid- and post-bond stack testing is proposed in [Marinissen10a]. This architecture facilitates modular testing, in which the various dies, their cores, the inter-die TSV-based interconnects, and the external I/Os can be tested as separate modules. This helps to achieve an optimal test flow for various 3D-SIC designs. The proposed architecture is based on the existing DfT features at the core, die, and product level. A die-level wrapper which can be based on either IEEE Std 1500 or IEEE Std 1149.1 is proposed [Marinissen10a].

A DfT architecture based on a modular test paradigm for 3D-SIC is proposed in [Marinissen10b]. Different dies, their cores, the TSVs, and the external I/Os can be tested individually. The proposed architecture is based on existing DfT hardware at the core, die, and product level. Die-level wrapper compatible with IEEE 1500 are supported. The proposed DfT includes dedicated probe pads on the non-bottom dies to facilitate pre-bond testing. Moreover, TSVs working as "test escalators" for routing test control and data signals up and down during mid- and post-bond testing are supported. Furthermore, a hierarchical "wrapper instruction register" chain can be included in the design [Marinissen10b]. Some of the test techniques and DfT features are further discussed in [Plas11].

Challenges in testing of 3D-SIC for manufacturing defects and their potential solutions are discussed in [Marinissen10c]. These are divided into the following categories: test flow, test data, and test access. Examples for interconnect defects, including voids in TSVs and misaligned microbumps are discussed [Marinissen10c].

The die-stacking steps that include thinning, alignment, and bonding may introduce defects. Therefore, the partial stacks (mid-bond) and the complete stacks (post-bond) may need testing. A test architecture optimization technique for 3D-SIC is proposed in [Noia10a] to minimize the test application time for both mid- and post-bond test stages. It is demonstrated that an optimal DfT architecture considering these test stages is different compared with the situation that only the final test stage is considered [Noia10a]. 


\section{Chapter 3}

A DfT architecture optimization technique for 3D-SIC is proposed in [Noia10b]. It is shown that 3D-SIC with large and complex dies placed at the lower layers requires less test time than stacks with complex dies at higher layers [Noia10b].

Test challenges for 3D-SICs are discussed in [Marinissen12a, Noia11]. The need for standards like IEEE P1838 is discussed. P1838 consists of a test wrapper hardware and a description language for a test standard. This includes a generic die wrapper, which will create a standardized interface for each die in a stack. The wrapper design must enable pre-, mid-, and post-bond test access. A standard interface for each die in the stack is suggested. All these enables partial and complete stack tests, including dieexternal tests [Noia11].

Another DfT architecture for 3D-SIC is proposed in [Marinissen12b]. It supports modular testing, meaning different dies, cores, TSV-based interconnects, and external I/Os can be tested during the relevant pre-, midor post-bond stages. The proposed architecture makes it possible to optimize the test flow under various conditions. It also provides yield monitoring and first-order fault diagnosis [Marinissen12b].

A DfT architectural optimization for 3D-SIC is proposed in [Noia12] to minimize the test application time for mid- or post-bond testing. Optimal architecture and the corresponding test schedule are obtained for a scenario where only the post-bond testing is performed. It is demonstrated that the optimal architecture and schedule are different for the scenario where a mid-bond testing is added to the existing post-bond tests [Noia12].

The optimal test flow for 3D-SIC is studied in [Taouil12]. A framework that embodies different test flows for die to wafer bonding paradigms is introduced. The cost associated with a range of test flows is assessed for several die yield and stack size alternatives. It is shown that the inclusion of pre- and mid-bond testing potentially reduces the overall cost [Taouil12].

\subsection{Temperature-Aware Test Scheduling}

Without simulating the temperatures, the thermally safe schedules for advanced SoCs will be unnecessarily long. This is due to the large safety margins which are necessary when the temperature values are unknown. Prior to the actual test (during the schedule generation) our knowledge of 
the actual temperatures during testing, without simulating the temperatures, will be severely limited. Therefore, temperature-aware test scheduling techniques that use a kind of temperature simulation are introduced.

A temperature-aware scheduling technique is proposed in [He06b]. The objective is to minimize the test application time and the constraints include keeping the temperature under a safe limit. Test partitioning is supported and the time between two consecutive partitions can be utilized for cooling. Interleaving allows tests for other cores to be performed while a hot core is interrupted for cooling. This allows for efficient TAM utilization and a short schedule is achieved. The problem is formulated as a combinatorial optimization and a Constraint Logic Programming (CLP) formulation is used to solve it [He06b]. A faster heuristic-based approach for this purpose is later on proposed in [He07].

The power impact of scan chain testing is studied in [Bild08]. It is shown that the scan-chain power consumption is considerably higher for at-speed testing compared to the operational mode. An exact test schedule optimization for minimization of test application time under temperature constraints is introduced. This exact approach could be slow for practical purposes and therefore a fast heuristic-based approach is proposed [Bild08].

A temperature-simulation driven test scheduling algorithm is proposed in [He08a]. Instantaneous simulated temperatures are used to guide the partitioning of the tests and lengths of the cooling intervals. Interleaving of tests for different cores is supported to achieve a high TAM utilization [He08a].

A partitioning and interleaving approach is introduced in [He08b]. The suggested method formulates the number of test partitions and the length of cooling intervals into an optimization problem and then uses constrained logic programming to solve it. The temperature is simulated using HotSpot [Huang06] and is constrained to avoid overheating. Since constrained logic programming is too slow to handle long tests, a heuristic is proposed for the test scheduling [He08b].

A partition-based temperature-aware test scheduling algorithm is proposed in [Yao09, Yao11a]. Tests are partitioned and the proper start time for each partition is defined as a decision variable. The optimization goal is to 
achieve a short test application time under the temperature constraints. A superposition-based temperature simulation scheme is proposed. The actual temperature simulation is performed for each partition only once at the very beginning using HotSpot [Huang06]. Later on these simulated temperatures are combined based on the superposition principle in order to obtain the temperature for different situations [Yao09, Yao11a].

A temperature-aware combined TAM design and test scheduling technique is proposed in [Yu09]. The proposed approach supports cycle-accurate temperature simulation as well as test partitioning and interleaving. Maximal TAM size and maximal safe temperature are given as constraints. A heuristic-based approach is used to minimize the test application time. In addition to the temperature simulations, the heuristic is guided by the power density and test application time of individual partitions [Yu09].

A temperature-aware test scheduling technique supporting the abort on first fail testing approach is proposed in [He09]. In such an approach testing is terminated as soon as a defect is detected. Therefore, the defect probabilities must be known before the scheduling. The proposed test scheduling technique supports partitioning and interleaving of tests. The proposed algorithm uses the simulated temperatures to guide the partitioning of tests and to determine the duration of the cooling intervals. The objective is to minimize the expected test application time while the temperatures of the cores are kept below the thermal safety limit [He09].

A temperature-driven test access routing and test scheduling for threedimensional $\mathrm{SoC}$ is introduced in [Chandran09]. Three dimensional design of DfT features combined with partition-based test scheduling is studied. The proposed temperature-aware technique minimizes the test application time while constraints on the available hardware resources are taken into account [Chandran09].

In [Vinay10], it is shown that 3D-SICs may rapidly become too hot since the thermal resistance between dies located at the middle of the stack and the heat sink is large. A temperature aware test scheduling technique is, then, proposed [Vinay10]. The proposed approach focuses on vertical temperature distribution in the 3D IC to avoid overheating. Moreover, a new test partitioning scheme is proposed based on the power variations. The proposed techniques consist of heuristics aiming at minimizing the test application time. The proposed thermal model is a linear RC-model that focuses on vertical temperature effects. It is demonstrated that the proposed 
technique can achieve a uniform vertical temperature distribution [Vinay10].

A test partitioning method for temperature-aware testing of 3D-SIC is proposed in [Millican14]. The objective is to generate a test schedule with a minimal test application time under the thermal-safety constraints. The partitions are determined based on a partitioning temperature so that the temperature within a partition does not vary too much [Millican14].

A test scheduling technique for 3D-SICs based on a session-less approach is proposed in [Flottes15]. Testing start times are formulated as the decision variables and test application time is minimized. A set of constraints including TAM availability, power budget, and thermal limits must be respected. A greedy heuristic is proposed and experimentally evaluated in [Flottes15]. The session-less approach generates shorter schedules compared with the session-based ones. Session-based approaches afford to find the optimal schedule while session-less approaches usually cannot find the exact optimum. The proposed heuristic can find a near optimal solution for large problem sizes resulted from session-less approaches [Flottes15].

\subsection{Process Variation Effects on Power and Temperature}

Process variation causes uncertainty in circuit parameters including the electric currents and therefore the dissipated power. Variations in the dissipated power values will result in temperature variations. This means that the temperature for two different fabricated instances of the same entity (e.g., an identical core design) will be different.

Consider a homogeneous multi-core SoC design. Assume that all the cores are executing exactly the same tasks with identical memory and resource access patterns (also identical state and input data). Assume that all the cores started from the ambient temperature (i.e., identical initial conditions) and the voltages are precisely equal. Assume that there is not heat transfer among the cores and the cores cooling capabilities are designed to be identical. The difference in their working temperatures is due to the so called intra-die variations ${ }^{1}$.

\footnotetext{
${ }^{1}$ Intra-die and inter-die variations are formally defined based on the concept of temperature error that will be introduced in chapter 4 .
} 


\section{Chapter 3}

Now consider two single-core SoCs with the same design. Assume that they are executing exactly the same tasks with identical memory and resource access patterns (also identical state and input data). Assume that both ICs started from the ambient temperature (i.e., identical initial conditions) and the voltages are precisely equal. The difference in their working temperatures is due to the so called inter-die variations.

The impact of process variation on leakage power for a $0.18 \mu \mathrm{m}$ Complementary Metal Oxide Semiconductor (CMOS) technology is studied in [Srivastava02]. It is shown that the process variation can drastically affect the leakage current. Based on Monte Carlo simulations an analytical model for estimating the average and the standard deviation of the leakage current is developed. It is then demonstrated that the average leakage obtained by taking the PV into account is significantly different from the leakage predicted by the deterministic models [Srivastava02].

Process variation is a major challenge for designing with technology nodes smaller than 90nm [Borkar03]. Large variations in voltage, current, power, temperature, and delay are expected. PV causes serious difficulties in designing advanced electronics and to address these difficulties a shift in the design paradigm, from existing deterministic approaches to adaptive or stochastic approaches (either probabilistic or statistical) is necessary [Borkar03].

A method for estimating the leakage current variations due to $\mathrm{PV}$ is proposed in [Rao03]. The problem is analyzed for both inter- and intra-die variations and a closed form Probability Density Function (PDF) for calculating the leakage current is developed. Distributions of individual gate's leakage currents are then combined to calculate the average and variance for a whole design. The closed form results are then validated against a set of Monte Carlo simulations [Rao03].

A stochastic approach for leakage power minimization based on dual $\mathrm{Vth}^{2}$ technologies considering PV is proposed in [Liu04]. A statistical model of $\mathrm{PV}$ is used in this stochastic optimization. Probabilistic analytical models are then developed to predict the impact of PV on the leakage power and

${ }^{2}$ Vth is the threshold voltage in CMOS-based technologies. One Vth value is sufficient to fabricate working ICs. However some manufacturers offer the possibility of using two different Vth values in a single die in order to achieve better performance/power trade-offs. 
delays. This model indicates that the existing non-probabilistic analysis significantly (around $3 \times$ ) underestimates the leakage power. Based on the proposed model the value of the second $\mathrm{Vth}$ is optimized considering the PV [Liu04].

A different stochastic Dual-Vth optimization technique considering PV is proposed in [Srivastava04]. It is shown that the deterministic methods are not appropriate in the presence of large process variations. The proposed stochastic approach can improve the leakage by $15-35 \%$ compared with the traditional deterministic approaches [Srivastava04].

A method for analyzing the leakage power under intra-die process variations is proposed in [Chang05]. A lognormal distribution is used to approximate the leakage current of individual gates and the overall leakage of a die is determined by combining these lognormal distributions. Both subthreshold leakage and gate tunneling leakage are considered [Chang05].

The leakage power is very sensitive to process variations and therefore PV results in large temperature variations [Choi07]. The temperature variations in FinFET circuits are affected by both the channel length variations as well as the body thickness variations. The temperature variation caused by PV are assessed using Monte Carlo simulations combined with temperature simulations. The simulations show that circuits with large switching activity suffer from larger temperature variations. This is due to larger static power as a result of the higher temperature caused by large switching activity. It is shown that under a moderate process variation (e.g., $3 \sigma=10 \%$ for channel length and body thickness) thermal runaway can occur in more than $15 \%$ of chips in a $28 \mathrm{~nm}$ FinFET technology [Choi07].

Process variation is caused by various reasons including [Nowka08]:

- imprecisions in alignment, rotation and magnification (lithography);

- interference effects from neighboring shapes (lithography);

- fluctuations in the photon absorption positions (lithography);

- fluctuations in the dosage of chemicals used for etching and treatment;

- random dopant fluctuation;

- gate oxide thickness fluctuation;

- Chemical Mechanical Polishing (CMP) unevenness; 


\section{Chapter 3}

Gate oxide thickness variation results in variation in the threshold voltage and consequently in variations in the static power dissipation [Nowka08].

Some of the stochastic approaches for dealing with the PV (e.g., statistical static timing analysis) cannot handle the dynamic changes during operation [Ganapathy10]. A new method based on multivariate regression is proposed to model the temporal delay variations under PV. Such variations are related to temperature variations [Ganapathy10].

On-chip temperature sensors are used to achieve a temperature-aware test scheduling and reduce the test application time compared to a static schedule [Yao11c]. Due to large PV the estimated test power values can be very different from the actual ones. Consequently, the estimated temperatures during the offline scheduling phase (prior to the actual test) can be inaccurate. A test architecture that supports dynamic test scheduling is assumed. A heuristic is suggested to generate the static schedule that the method is based on. Then a dynamic test scheduling method using on-chip temperature sensors is proposed [Yao11c].

Dynamic reliability management techniques dynamically tune a system's operation based on the tradeoff between performance and reliability. The proposed method in [Zhuo10] takes the spatial and temporal variations (including PV) into account. Moreover, the proposed technique is workload-aware meaning that it reacts to sudden workload variations [Zhuo10].

A flexible probabilistic framework for evaluation of the transient power and temperature variations under large PV is introduced in [Ukhov14a]. This models the probability functions of the fluctuating parameters. The proposed technique captures the power and temperature variations in a closed-form analytical model [Ukhov14a].

A system-level framework for the analysis of temperature-related failures affected by PV is proposed in [Ukhov14b]. This includes a probabilistic technique for dynamic steady-state temperature modeling and a closedform stochastic modeling of the system. Temperature cycling induced aging is analyzed in presence of large PV. The proposed technique minimizes the expected energy consumption under performance, temperature, and reliability constraints [Ukhov14b]. 


\subsection{Multi-Temperature Testing}

A detailed study of defects found in a commercial microprocessor is performed in [Needham98]. For this high production volume microprocessors, the manufacturing tests are designed so that very small testescape statistics are achieved. Some of the escaped defective devices are rigorously analyzed to find out the defect's type, its electrical effect, and the possible methods to detect such defects easily. Lessons learned from these defects in combination with the technology trends enables the authors to determine what should be done to achieve and maintain high-quality manufacturing and test. This includes defects that can be detected by multitemperature testing but are otherwise hard to detect [Needham98].

The conclusions from a failure analysis study in SEMATECH ${ }^{3}$ is reported in [Nigh98]. The testing procedures, IC stressing to achieve high reliability, characterization of the defects, fault diagnosis, and physical analysis are presented for a number of devices. Testing at different temperatures is discussed in [Nigh98].

Delay-defect test-escapes are examined in [Tseng00]. Among these defects, detecting the defects that are caused by high resistance interconnects are very challenging. A cold testing technique that performs the test at low temperature can help. Cold testing is in particular effective for detecting the silicide open defects [Tseng00].

The behavior of resistive open defects are studied in [Li01]. Temperaturedependent defects that motivate multi-temperature testing are discussed. The effects of temperature on testing are investigated and an effective testing method for resistive opens is presented [Li01]. It is suggested that by knowing the location of such defects and the materials involved in those defects, the proper testing temperatures can be found and the appropriate test patterns can be generated [Li01]. Such testing temperatures and test patterns are used to perform multi-temperature testing.

Parametric failures are more frequent in advanced electronics, where the feature size is very small [Segura02]. These hard to detect failures are experimentally studied and classified. Multi-parameter test strategies are

\footnotetext{
${ }^{3}$ SEMiconductor MAnufacturing TECHnology (SEMATECH) is a research consortium for IC manufacturing. http://public.sematech.org/ (May 2015)
} 


\section{Chapter 3}

suggested to address this complex test problem. These issues are also discussed in [Segura04].

Due to very small copper interconnect dimensions in advanced electronics, physical failure analysis is needed to address the potential defects. Failure localization and defect analysis are challenges for copper inlaid technologies [Zschech02]. Failure localization and analysis using $\mathrm{FIB} / \mathrm{SEM}^{4}$ and $\mathrm{TEM}^{5}$ are described. The voids in copper interconnects and buried residuals in vias are studied in [Zschech02]. These defects result in temperature sensitive defects that necessitate multi-temperature testing.

Multi-temperature testing is analyzed based on experimental data from $0.25 \mu \mathrm{m}$ and $0.18 \mu \mathrm{m}$ technologies, in [Long04]. Then, based on these data, a model is developed. This model is used to design new temperature-based tests to improve the test's quality. Temperature based test data are presented for a range of measurements including transistor characteristics needed to parameterize the model [Long04].

Some imperfections in the chip (e.g., some resistive opens or shorts) will not hinder the normal operation of the chip just after the fabrication, at the time that the manufacturing test is performed. But these imperfections are reliability threats because they are weak points in the circuit that wear out quickly and will lead to failures during the expected lifetime of the chip [Long04, Needham98]. Some of these imperfections can be identified by multi-temperature testing.

Performance outliers and defects are examined across the expected operating temperature range [Schuermyer04]. Minimum testing requirements to detect temperature dependent outliers at wafer sort and final test are investigated. This is based on data from a $0.18 \mu \mathrm{m}$ technology obtained at $30^{\circ} \mathrm{C}$ and $85^{\circ} \mathrm{C}$. It is argued that temperature-sensitive defects are expected to become more frequent in advanced technologies and, therefore, it is important to develop effective test methodologies for them [Schuermyer04].

${ }^{4}$ Focused Ion Beam (FIB) is a visualization technique used for site-specific analysis of materials. It is similar to a Scanning Electron Microscope (SEM).

${ }^{5}$ Transmission Electron Microscopy is a visualization technique based on electron beams transmitted through the object. 
Resistive defects are important in advanced electronics but they need special conditions in order to be detected. The detection approaches for resistive bridging (short) defects are studied in [Engelke08, Kundu05]. Testing at low temperatures may help. Resistive bridge defects are studied under multiple environmental conditions. Moreover, imperfections that are not defects at nominal conditions but could deteriorate and become earlylife failures are studied. It is suggested that there exist appropriate combinations of these tests that provide satisfactory test coverage for different types of defects [Engelke08, Kundu05].

The performance of advanced electronics that are made by deep submicron technologies can be affected by phenomena that were previously considered not to be important [Wu10]. One of such phenomena is Inversed Temperature Dependence (ITD). ITD means that the delay of electronics may decrease with temperature; against the traditional understanding that the electronics delay increases with the temperature. The reason for this phenomenon is the smaller threshold voltage (implying faster operation) at high temperatures which dominates the smaller carrier mobility (implying slower operation) at high temperatures. Traditionally, delays are checked at two temperature corners, one representing the best case (used to happen at low temperatures) and the other representing the worst case (used to happen at high temperatures). For advanced electronics which experience ITD the high temperature may not correspond to worstcase delays [Wu10].

Advanced electronics require new types of testing, like temperaturetesting, in order to maintain high product quality. The effect of test temperature on the quality of the tests is studied in [Jagan10]. A low-cost alternative to temperature testing is proposed. Moreover, the proposed technique determines the appropriate test conditions for the best test quality and lowest cost. The proposed test flow is experimentally evaluated on an industrial-standard die. A defect's behavior at low-temperature is studied using Shmoo plots $^{6}$ [Jagan10].

The need for testing advanced core-based SoCs at different temperatures is discussed in [He10]. Then a multi-temperature test scheduling for SoCs is introduced which assumes that tests should be applied inside predefined

\footnotetext{
${ }^{6}$ Shmoo plot is a graphical representation of a device's response to a range of conditions and inputs (e.g., temperature and voltage).
} 


\section{Chapter 3}

temperature ranges. The proposed scheduling approach minimizes the test application time and ensures that tests are only applied within the valid temperature ranges [He10]. For this purpose the temperatures of the cores are simulated. Based on the simulated temperatures, heating or cooling intervals are introduced into the schedule [He10]. The proposed method is based on partitioning and interleaving and therefore when a core is having its cooling interval, other cores may utilize the test access mechanism's capacity that has been just made available [He10].

Another multi-temperature test scheduling scheme for SoCs is introduced in [Yaol1b]. It assumes that tests should be applied at their specified temperature ranges (can be different from each other). Cooling intervals are inserted if the core temperature is too high and heating stimuli are applied when the temperature must be increased in order to meet the required temperature conditions for correct testing [Yao11b]. The proposed scheduling approach in [Yao11b] is based on list scheduling and assumes that tests run always to completion without any interrupts. The initial list order is determined based on the lowest valid temperatures for the tests. The list schedule determines the earliest start times for tests. The test application time is minimized and it is ensured that tests are applied within correct temperature ranges [Yao11b].

\subsection{Temperature Gradients and Burn-In}

The presence of voids in $\mathrm{Cu}$ structures results in important reliability issues for advanced electronics. The mechanical stress in the interface between the $\mathrm{Cu}$ and capping layers ${ }^{7}$ is experimentally investigated in [Murray12]. In technologies that deposit the cap at lower temperatures, the $\mathrm{Cu}$ does not show considerable depth-dependent stress. Even though an annealing technique can decrease the stress gradient, when the temperature goes back to the room temperature after being close to the deposition temperature, the gradient appears again [Murray12].

A mechanism that causes defect formation in metallization (e.g., interconnects) under fast temperature cycle stress is studied in [Smorodin08]. The lateral temperature distribution (i.e. temperature gradient) causes an accumulating plastic deformation of the metal layer.

\footnotetext{
${ }^{7}$ Capping layer is the electrical insulation used to insulate different interconnects and wire lines in a die.
} 
Large deformations occur in sites which experience large temperature gradients [Smorodin08].

Burn-in is used to accelerate various aging and failure mechanisms so that the imperfections that may cause infant mortality are detected before the product is shipped [Miller01]. Burn-in acceleration is achieved by imposing high temperatures, high voltages, high toggle rate, and/or high current density on the circuit under test. One of the traditional test flows is a burn-in following a test by ATE and then again an ATE test. It is suggested that this first ATE testing and the burn-in can be combined into a hybrid burn-in, improving the overall test process [Miller01].

Reliability predictions are based on a number of sources of information including in-service field return data and physics of failure [Bayle10]. Previously, predictions were mainly based on empirical data but recently physics of failure is being incorporated into the lifetime models. The existing reliability models usually are based on steady-state temperature. A new methodology that combines several recent works that address new mechanisms of failure (e.g., hot carrier and delamination) is proposed for aeronautic applications [Bayle10].

\subsection{Testing for Delay-Related Defects}

Gradients and early life failures are discussed above. Gradients have some other negative consequences. One of them is discussed in the following. Different temperatures on different sites mean that the signal delays (that depend on the sites that a signals route passes through) will have different delays. This can cause delay-related faults that must be detected using atspeed and delay tests, as discussed below.

Some floating-point data-paths are developed for graphics and simulation applications in [Hagihara97] using a 0.35-micron technology. They are designed to be embedded in a vector pipelined processor for use in supercomputers. An online test technique is introduced to improve the reliability under actual operating conditions that includes temperaturegradients. The technique makes it possible to detect delay faults as well as the static faults (i.e., normal defects) [Hagihara97].

For advanced SoCs containing millions of gates and working with frequency in gigahertz range, at-speed test is crucial [Ahmed05]. The launch-off-shift method has some advantages over the launch-off-capture 
technique but requires perfect transition fault testing with regard to atspeed scan enable signal. A scan-based at-speed test is introduced in [Ahmed05] that is based on multiple local fast scan enable signals. The scan enable control information is sent as test data through the scan. Moreover, an innovative scan cell is introduced to generate the fast local scan enable signal [Ahmed05].

Keeping the power consumption checked during at-speed testing is investigated in [Ko08]. A common practice is to divide the scan chain to control shift power by activating mutually exclusive flip-flops at different times during the scan cycle. However, the existing automatic test pattern generation techniques do not provide means to control the capture power. Therefore, a new scan chain division algorithm is introduced in [Ko08]. It takes into account the signal dependencies and partitions the circuit such that both shift and capture power can be reduced. Moreover, a technique for utilizing partial scan combined with the scan chain divisions is proposed [Ko08].

Test power constraints are usually due to the power delivery limitations. These limitation could be due to the limited capability of the power network in the device or the limited test equipment's capability [Zhao10]. Excessive switching activity during launch-to-capture cycle in delay test causes many problems. These include overkill of dies and damaging the ATEs' probes [Zhao10]. A fast technique for finding the high-power patterns and replacing them with power-safe ones is introduced. Being high power is defined in relation with ATEs' power limit. The proposed technique takes the spatial and electrical properties of the power distribution network into account [Zhao10].

At-speed scan-based testing may be affected by launch safety issues [Wen11]. This means that the test results are incorrect because of excessive launch switching activity which is related to the test stimulus launch in the at-speed test cycle. A power-aware test generation flow is proposed in [Wen11] to guarantee a safe launch. The proposed rescue and mask scheme targets the excessive switching activity around the long path that the test vector targets. The rescue phase reduces the power. If the new power value is still too large the test responses are masked. The proposed approach guarantees launch safety with a negligible impact on test quality and costs [Wen11]. 
Launch-off-capture and launch-off-shift are the two major at-speed scanbased delay testing techniques [Bosio11]. Usually, launch-off-shift offers higher fault coverage and faster test than launch-off-capture technique. However, it suffers from higher peak power consumption in the launch-tocapture cycle. A don't care filling technique is proposed to adjust peak power consumption in relation with the power consumption in functional mode. The objective is to generate a test set with peak power values similar to the functional power [Bosio11].

3D-SICs are manufactured based on micro-bumps that connect two of the stack dies together [Shibin15]. Moreover, TSVs provide electrical connections between the front- and back-side of a die. It is reported that imec $^{8}$ and Cadence ${ }^{9}$ have developed a 3D-DfT architecture based on DfT die wrappers [Shibin15]. The TSVs and micro-bumps can be tested for static defects (e.g., hard opens and shorts) by existing techniques. Such interconnects might also be affected by resistive opens and shorts, which usually manifest themselves as delay faults. The reported 3D-DfT is recently enhanced to support at-speed transition-based delay-fault testing. The reported framework works with mission-mode speed and employs the already existing clock distribution network [Shibin15].

A delay fault simulator for combinational circuits is developed in [Manikandan11]. It helps to develop the delay tests faster. The experiments consider K-longest path sets of ISCAS'85 benchmarks. A large number of single input test patterns are repeated for a number of times to achieve statistically valid data. The proposed technique is reported to provide good fault coverage and 20\% speed-up [Manikandan11].

A transient fault injection technique for simulation-based fault-injection in advanced SoCs is proposed in [Rohani13]. The proposed technique can inject a wide range of faults without modifying the top-level design. Moreover, the proposed technique is fast. Two experimental case studies show that the proposed technique reduces the CPU time by $10 \%$ compared with other similar techniques [Rohani13].

\footnotetext{
${ }^{8}$ Interuniversity MicroElectronics Centre (IMEC) is an electronics research center. http://www2.imec.be/be en/home.html (May 2015)

${ }^{9}$ Cadence Design Systems Inc is an electronic design automation company. http://www.cadence.com/cadence/Pages/default.aspx (May 2015)
} 


\section{Chapter 3}

\subsection{Temperature Cycling}

It has been known for long that varying mechanical stress in metals will result in metal fatigue and consequently lead to metal structure failure. The varying stress has various causes, including mechanical load variations and temperature fluctuations (i.e., cycling). Accurate estimates for the effect of fluctuations help to know the lifetime of a part. This enables a better (simulation-based) design of the parts. Besides it enables the timely replacement of the parts which translates into a safe and cost-efficient maintenance of the structure (e.g., a ship or a plane). A well-known approach for estimating this aging effect is the Rainflow counting algorithm proposed in [Matsuishi68].

Cycle counting methods (e.g., Rainflow algorithm) identify equivalent full and half cycles within the irregular load profile [Musallam12]. Then the cycle-based lifetime models can be used. The original Rainflow algorithm is applied offline meaning that the whole temperature or load profile over the desired operational time period must exist before it can start. Therefore, it cannot be used for applications that need it in real time. An online counting algorithm which uses a stack-based implementation is proposed in [Musallam12] and used in this thesis.

Time dependent average temperature effect is combined with the results from the Rainflow algorithm in a single lifetime model in [GopiReddy14]. A month long load profile is used as a test profile to estimate temperatures in a power system for reactive compensation of load [GopiReddy14].

Insulated Gate Bipolar Transistor (IGBT) is a power-electronic device with a relatively wide range of applications including automotive traction [Held97]. Such applications require high reliability in particular under power cycling. Power cycling causes temperature changes which lead to mechanical stress. This can lead to defects such as lifting of bond wires. A fast cycling test that activates the failure mechanism is suggested to enable reproduction of millions of cycles in a short time. The effectiveness of the proposed approach is verified by a mechanical analysis. A model is developed to relate the number of cycles-to-failure to the magnitude of temperature changes [Held97].

Another mechanism affecting the lifetime of electronic devices can be modeled by the Arrhenius equation. An important parameter is the 
activation energy ${ }^{10}$ that relates to the working temperature [Groebel01]. Accelerated-test data are experimentally obtained and then used to accurately estimate the activation energy. A software package dedicated to this experimental approach is used to speed up the process. Acceleratedlife test data are acquired for a thermally stressed hard-drive system and analyzed using the Arrhenius-Weibull model. The Arrhenius model parameters are estimated using a maximum likelihood algorithm. Then, the activation energy is estimated [Groebel01].

A few procedures for extracting the statistical parameters of temperature cycling experienced by power devices for different mission profiles (e.g., how an electric vehicle is driven) are investigated [Ciappa03a, Ciappa03b]. These statistical models help to design efficient accelerated tests and to fine-tune the lifetime models. A precise lifetime model that takes into account the creep ${ }^{11}$ experienced by compliant materials under thermal cycles is developed in [Ciappa03a, Ciappa03b].

Electronics reliability is affected by the average working temperature as well as the temperature cycling effect [Hirschmann06, Hirschmann07]. Temperature simulation is used to estimate the dynamic temperature values. Temperature cycling plays an important role in lifetime prediction models. A technique for detecting all relevant temperature cycles is developed in [Hirschmann06, Hirschmann07].

A lifetime model for solder joints under cyclic thermal-mechanical loading is developed in [Lu07]. The model combines a linear damage accumulation

10 Activation energy is a term primarily used in chemistry to approximately describe the minimum energy required to start (activate) a reaction. The reaction is modeled by Arrhenius equation that has the activation energy as a main parameter. In other situations that are not exactly a chemical reaction, but the Arrhenius equation is used for pure modelling purposes, the term "activation energy" is nevertheless used for the main parameter in the model disregarding its original namesake.

11 Creep or cold flow is when a solid material moves slowly or deforms (permanently) under mechanical stresses. Exposure to stress during a relatively long period of time can do this. Heat exacerbate creep. The amount of stress that can cause this is less than the value needed to literally bend the material instantaneously. 


\section{Chapter 3}

with the effect of accumulated plastic strain ${ }^{12}$. The model is then used to predict the lifetime for a power module that operates under mixed cyclic loading conditions (e.g., a train's traction system) [Lu07].

A solder fatigue model based on a modified Coffin-Manson approach is proposed in [Vasudevan08]. The proposed model is evaluated using temperature cycling experiments. The experimental data for various types of packages and sockets have been used. The proposed model's error is reported to be less than $6 \%$ [Vasudevan08].

Through silicon vias reliability issues are investigated in [Kamto09]. The experiments performed using a technology based on deep reactive ion etching show that TSVs with tapered sidewalls can be formed. The TSVs experience temperature cycling. Considerable increase in the electrical resistance of the paths going through TSVs is observed after temperature cycling. Perfect TSVs only show small increases in resistance for 200 cycles. Moreover, small changes in resistance are observed when TSVs experience high temperatures for extended periods of time [Kamto09].

The acceleration factor for solder depends on the magnitude of temperature changes, dwell times, ramp rates, actual values of temperature extremes, and the type of package [Syed10]. A lifetime model that relates the actual real-life lifetime with accelerated lifetime based on a number of factors including temperature cycling is proposed [Syed10].

The relation between the initial electrical resistance of TSVs and failures due to temperature cycling as well as electromigration is studied in [Frank10]. Physical analysis shows that a carbon impurity layer at one end of the problematic TSVs is developed. This impurity results in failure under temperature cycling while it has no correlation with defects caused by electromigration [Frank10].

The thermal stress distribution for a TSV array is studied in [Kuo11, Kuo12]. In TSV-based structures, there are large coefficient of thermal expansion (CTE) mismatches between silicon substrate, dielectric material, and filled metal. Therefore, the thermal stress at the interface of materials is large and results in material failure or delamination. The

${ }^{12}$ Strain within material is either elastic or plastic. While elastic strain only can cause a reversible distortion, the plastic strain can result in non-reversible deformation including cracking of the material. 
thermal-mechanical stress distribution of a TSV array model under the accelerated temperature cycling is investigated by a finite element approach. The surface area between TSVs is squeezed at high temperature and this results in compressive stress at the surface area. The analysis shows that large stress occurs around pads. This may result in failure or delamination of TSV pads. The simulations indicate that for larger pads that result in smaller space between TSVs the stress is larger. Smaller pads experience higher stress close to the pad corners but the stress is smaller at the middle of bottom pad. The proposed analysis technique helps to identify possible failure regions in the TSV structure [Kuo11, Kuo12].

Large shear stress ${ }^{13}$ develops at the interfaces between different materials during temperature cycling, especially if the difference between their CTE is large [Kumar12]. The shear stress may cause interfaces to slide by a diffusional process. This results in relative dimensional changes in the materials. This is a reliability risk for TSV based structures which not only suffer from temperature cycling but also convey large current densities. Experimental results demonstrate interfacial sliding caused by temperature cycling in presence of electric current. The presence of current moved the affected area in the direction of electron flow. This leads to exacerbated protrusion (or intrusion) of TSV relative to the temperature cycling only situation (when the electric current is negligible) [Kumar12].

The effect of temperature cycling as well as some other thermal phenomena on the performance of TSV based electronics is studied in [Cherman12]. The transistor performance is affected by the stress induced by the TSV. It is reported that high working temperature increases the TSV-induced stress while temperature cycling decreases this stress. These stress variations may be due to the TSV creep [Cherman12].

A study for understanding the effect of temperature cycling on the signal integrity for TSV based electronics is conducted in [Okoro12]. Radio frequency signals are used to detect discontinuities in the isolation liner around the TSV metal body. Signal degradation increases with temperature cycling. Atomic Force Microscopy (AFM) showed that void formation and growth in the isolation liner is the root cause [Okoro12].

\footnotetext{
${ }^{13}$ Shear stress is the stress force parallel to the surface of a material. It is different from the normal stress which acts vertical to the surface.
} 
System reliability is affected by a number of factors including the amount of temperature cycling [Chantem13]. Task assignment and scheduling may help to even out the core wears in an advanced multi-core system. A dynamically-activated task assignment and scheduling algorithm that prolongs system lifetime is proposed in [Chantem13].

Thermal-mechanical failures of TSVs including the TSV protrusions from the die surface are studied in [Zhang13]. The TSV protrusions are observed after wafer bonding, thinning, and TSV revealing. TSV protrusion on the backside is affected by temperature cycling. Protrusion magnitude can be fitted to an exponential model which suggests a grain boundary diffusion mechanism might be behind it [Zhang13].

Temperature-related mechanical stress in TSV structures is studied in [Jiang14]. An X-ray micro-beam diffraction visualization technique is used to observe the stress and deformation in TSV with submicron resolution. Local plasticity in TSV and the deformation induced by thermal stresses are investigated using this technique. Grain growth in TSV metal body affects the stress relaxation during temperature cycling and consequently the residual stress and plasticity in the TSV structure [Jiang14].

\subsection{Test Reordering}

During the test, the power consumption of the circuit under test may exceed its power rating, as discussed before. A test power reduction technique based on test vector ordering is proposed in [Chakravarty94, Dabholkar98]. The objective is to minimize the tests average switching activity. It is demonstrated that the test ordering problem is NP-hard. Consequently, a greedy approach for finding a low-power test order is proposed. An elaborate power model based on the transition count in the scan chain is used [Chakravarty94, Dabholkar98].

Another test planning technique is proposed in [Girard97] to reorder the test vectors to minimize the switching activity of the circuit under test. A close connection between the actual number of transitions and the Hamming distance between tests is confirmed. Consequently, a fast algorithm to calculate Hamming distances is used instead of the actual transition count which is excessively time-consuming to calculate. A greedy heuristic is then used to find a low power test order [Girard97]. 
In safety-critical applications, the electronics are frequently tested (the test might be even in-field and even online) by Built-in Self-Test (BIST) modules [Flores99]. Online testing, in particular, can consume a large part of the overall power budget. A test ordering technique for power reduction is proposed in [Flores99]. The circuit-under-tests switching activities are approximated by Hamming distances between the subsequent tests. The problem is equivalent to a travelling salesman problem. The problem is simplified so that an ILP technique can be used. Moreover, a Christofides algorithm is employed to find a low-power test order [Flores99].

A method for reducing the test application time while respecting a power budget is proposed in [Rosinger02]. The method focuses on the test power peaks. These peak values depend on the order of the tests. The tests are reordered so that the power peaks for different cores are not overlapping. This leads to a minimized TAT under power constraints [Rosinger02]. The technique works as follows: First, power dissipation is minimized. Then, the current results are further improved by test application time minimization. When minimizing the test application time, the power is considered as a constraint [Rosinger02].

Testing during the burn-in process is a common practice since it reduces test and burn-in costs [Bahukud08a, Bahukud08b, Bahukud09]. However, power variations caused by scan-based testing may lead to large temperature fluctuations. This affects the accuracy of the burn-in process since the actual temperatures are not exactly known. Reducing power variations in order to reduce the temperature variations during burn-in is investigated in [Bahukud08a, Bahukud08b, Bahukud09]. The variation is reduced through test reordering. An ILP approach as well as a greedy algorithm are used to properly reorder the tests. An efficient transition counting method is proposed to rapidly estimate the test power values. Then, a heuristic-based test-pattern ordering technique is proposed to minimize the fluctuations in the power dissipation during test [Bahukud08a, Bahukud08b, Bahukud09].

Scan-based testing usually causes much larger switching compared to normal circuit operation [Tudu09]. This results in large power consumption which in turn leads to supply droop and yield loss. An efficient technique for test vector reordering to achieve an acceptably low peak power is proposed in [Tudu09]. The peak power values are represented by a complete directed graph. Consequently, a number of 


\section{Chapter 3}

graph based techniques are employed to reduce the peak power. Removing the edges with peak power larger than a certain threshold is one of the preprocessing techniques. After that, the remaining graph is searched for a Hamiltonian path. Other techniques, such as repeating a test, adding an allzero test, and adding an all-one test are also studied. The average power is also minimized under the peak power constraint [Tudu09]. 


\section{Chapter 4 Process-Variation Aware SoC Test Scheduling Techniques}

This chapter presents techniques to address the negative effects of process variation on the thermal issues during test. In advanced SoCs manufactured by deep submicron technologies, the portion and the absolute value of the temperature error induced by process variation $(\mathrm{PV})$ is considerable. The PV will cause large errors mainly due to power variations [Choi07]. Large error magnitudes directly translate into the need for larger safety margins and consequently excessively long test application times.

The usual offline test scheduling techniques are vulnerable to temperature errors since the error values are not known a priory. Therefore, the temperatures that are simulated offline could be very different compared with the actual temperatures. Since the actual temperatures are accessible during test through temperature sensing, an online scheduling alternative seems promising. However, online test scheduling has its own drawbacks, such as additional delays due to temperature readout times and run time overhead. As a compromise, an adaptive approach is proposed in this chapter to take advantage of both offline and online scheduling paradigms.

\subsection{Introduction}

Two process variation aware methods are proposed in [Aghaee10] in order to maximize the test throughput. One of these techniques is offline and the other is hybrid (quasi-static). The optimization objective (i.e., testing throughput) is defined to take the cost of the overheated chips into account in addition to the test application time. However, these techniques handle neither intra-die process variation nor temperature error fluctuations. In this section an adaptive test scheduling method is introduced which navigates the tests according to the intra-die process variation thermal effects and temporal deviations in thermal behavior of the chip. It makes 


\section{Chapter 4}

use of multiple on-chip temperature sensors to provide intra-die temperature information.

Integration of such sensors is already practical. For example Power5 is reported to have 24 sensors in year 2004 [Clabes04]. A variety of mechanisms to access the sensors during test are proposed in [Ieee14b, Yao11c]. The proposed approach in this thesis assumes an overhead for sensor access and tries to reduce the number of sensor accesses.

As mentioned in section 3.4, there are related sensor-based works in this area that support neither partitioning nor temperature-dependent leakage. The method proposed in this chapter is based on partitioning and interleaving, which reduces and/or utilizes the cooling times in order to decrease the overall test application time. It also handles the long and power intensive tests which are not thermally-safe. Moreover, temperature-dependent leakage is taken into account.

The proposed method generates a near optimal schedule tree at design time (offline-phase). During testing (online-phase), each chip traverses the schedule tree, starting from the tree's root and ending at one of the tree's leaves, depending on the actual temperatures. The schedule indicates when a core is testing and when it is in the cooling state. The order of the test sequences is untouched and the schedule tree's size (i.e., storage footprint) is small.

Traversing the schedule tree requires a very small delay overhead for jumping from one point in the schedule tables to another point. This way, the complexity is moved into the offline-phase and the memory/delay overhead of the online-phase is minor. To our knowledge, this is the first work to present an approach which incorporates the on-chip temperature sensors data, repetitively during test, in order to adapt to the temperature deviations caused by process variation and to achieve a superior test performance.

\subsection{Motivational Example}

Assume that there are two instances, $C_{w}$ and $C_{n}$, from a set of chips manufactured for a given design. When the temperature error between the actual temperature and the expected one is negligible, the temperatures of $C_{w}$ and $C_{n}$ during a test process are equal and the same offline test schedule $S_{1}$ is used for both of them. As illustrated in Figure 4.2.1a, both $C_{w}$ and $C_{n}$ 
are tested without overheating, since the test schedule includes cooling periods whenever the thermal simulator indicates that the chip temperature will exceed the limit.

Due to process variation, however, the thermal responses of the different chips to the same test sequence will be different. Now, assume that chip $C_{w}$ is warmer than expected, while chip $C_{n}$ behaves normally. As illustrated in Figure 4.2.1b, $C_{w}$ will overheat. To prevent this, a more conservative offline schedule $S_{2}$ has to be designed based on the thermal profile of $C_{w}$, for both chips, as illustrated in Figure 4.2.1c.

This new schedule will avoid overheating, but will lead to longer test application time $T A T_{2}$ compared with $T A T_{1}$. For chip $C_{n}$, this test application time is unnecessarily long, since the original schedule, $S_{1}$, in Figure 4.2.1a is a safe schedule for this particular chip. For a set of manufactured chips with large temperature variations, in order to generate a globally conservative offline schedule, the hottest chip will be used to

(a)

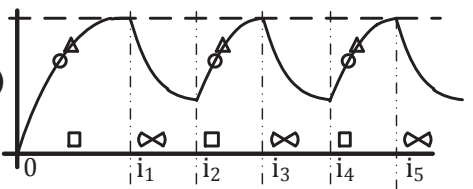

(b)

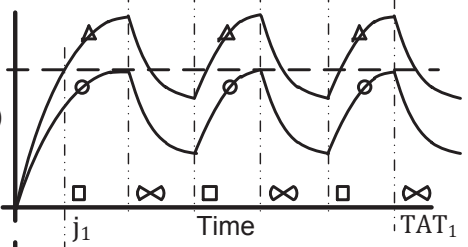

(c)

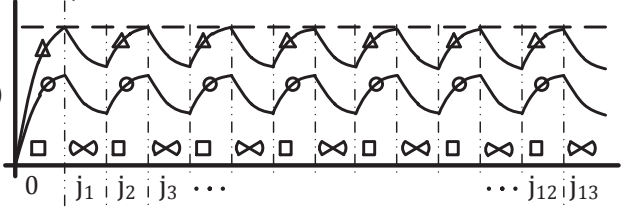

(d)

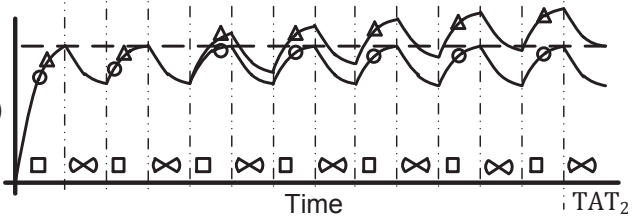

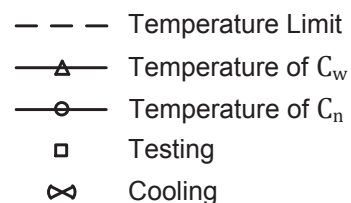

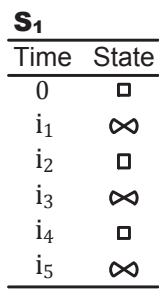

\begin{tabular}{cc}
$\mathbf{S}_{\mathbf{2}}$ & \\
\hline Time & State \\
\hline 0 & $\mathbf{0}$ \\
$\mathrm{j}_{1}$ & $\infty$ \\
$\mathrm{j}_{2}$ & $\square$ \\
$\vdots$ & $\vdots$ \\
$\mathrm{j}_{13}$ & $\infty$ \\
\hline
\end{tabular}

Figure 4.2.1 Test schedule examples

Temperature curves (a) when there is no temperature error; (b) when there is time-invariant temperature error and schedule $S_{1}$ is used; (c) when there is time-invariant temperature error and schedule $S_{2}$ is used; (d) when there is time-variant temperature error. (Curves are only illustrative.) 
determine the test schedule. This test schedule will introduce too long cooling periods for most of the chips, leading to an inefficient test process.

The hybrid technique presented in [Aghaee10] addresses the above problem with the help of a chip classification scheme. This scheme consists of several test schedules for different temperature error ranges. After applying a short test sequence, the actual temperature of the chip under test is measured using a sensor and depending on its value, the proper test schedule is selected. Therefore, the hotter chips will use a test schedule with more cooling, while the colder chips will have less cooling. The overheating issue is solved and the test application time will not be made unnecessarily long. This approach works fine under the assumption that the thermal behavior of the chips is time invariant (e.g., Figure 4.2.1a-c).

However, in the case of large process variation, the thermal behavior is time variant and the technique presented in [Aghaee10] will not be able to achieve high quality schedules. The variation of thermal response with time is illustrated in Figure 4.2.1d. In this case, the temperature of chip $C_{w}$ gradually lifts up, as compared to $\operatorname{chip} C_{n}$, and $C_{w}$ eventually overheats. A scheduling method capable of capturing temporal deviations is therefore required to deal with this new situation

The temperature behavior given in Figure 4.2.1d is captured in Figure 4.2.2a with more details. The lift up of the temperatures of chip $C_{w}$ starts at time $i_{3}$, as shown in Figure 4.2.2a. Since $C_{w}$ will only overheat after $i_{4}$, both chips can be safely tested with schedule $S_{1}$ up to $i_{4}$. At $i_{4}$, the actual temperature of the chip under test, $\theta^{4}$, can be obtained via sensors. The actual temperature can then be compared to a Threshold and the following two different situations can be identified:

$$
\begin{cases}C_{w} & \text { if } \theta^{4}>\text { Threshold } \\ C_{n} & \text { if } \quad \theta^{4} \leq \text { Threshold }\end{cases}
$$

For the rest of the test, after $i_{4}$, two dedicated schedules, $S_{2}$ and $S_{3}$, are generated in the offline phase for $C_{n}$ and $C_{w}$, respectively. Therefore, in the online phase the test of $C_{n}$ continues with schedule $S_{2}$, as in Figure 4.2.2a, and the test of $C_{w}$ continues with schedule $S_{3}$, as in Figure 4.2.2b.

In this illustrative example, at the end of $S_{1}$, the schedule does a branching to either $S_{2}$ or $S_{3}$ based on the actual temperature. This information and the branching condition can be captured in a branching table, $B_{1}$ in Figure 
4.2.2. As shown in Figure 4.2.2a, $C_{n}$ is tested initially with $S_{1}$ and then with $S_{2}$, while, as shown in Figure 4.2.2b, $C_{w}$ is initially tested with $S_{1}$ and then with a more conservative schedule, $S_{3}$.

The segments of the schedule which are executed sequentially without branching are called linear schedules. An adaptive test schedule consists therefore of a number of branching tables in addition to multiple linear schedule tables. Note that the original test sequences are saved elsewhere in an intact order without being duplicated.

Although the above illustrative example was about a single-core design, the focus of this thesis is on multi-core SoCs. It is assumed that, due to the intra-die process variation, each core has its own thermal behavior similar to what is described above for a chip. Moreover, multi-core designs usually
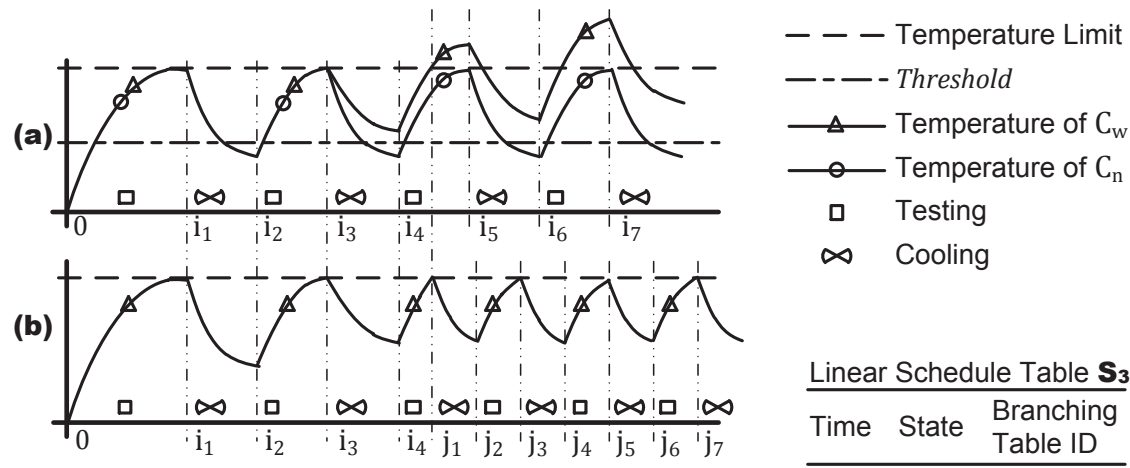

Linear Schedule Table $\mathbf{S}_{\mathbf{3}}$

\begin{tabular}{ccc}
\hline Time & State & $\begin{array}{l}\text { Branching } \\
\text { Table ID }\end{array}$ \\
\hline $\mathrm{j}_{1}$ & $\infty$ & - \\
$\mathrm{j}_{2}$ & $\square$ & - \\
$\mathrm{j}_{3}$ & $\infty$ & - \\
$\mathrm{j}_{4}$ & $\square$ & - \\
$\mathrm{j}_{5}$ & $\infty$ & - \\
$\mathrm{j}_{6}$ & $\square$ & - \\
$\mathrm{j}_{7}$ & $\infty$ & - \\
\hline
\end{tabular}

Linear Schedule Table $\mathbf{S}_{\mathbf{1}}$

\begin{tabular}{ccc}
\hline Time & State & $\begin{array}{c}\text { Branching } \\
\text { Table ID }\end{array}$ \\
\hline 0 & $\square$ & - \\
$\mathrm{i}_{1}$ & $\infty$ & - \\
$\mathrm{i}_{2}$ & $\square$ & - \\
$\mathrm{i}_{3}$ & $\infty$ & - \\
$\mathrm{i}_{4}$ & $\square$ & $\mathrm{B}_{1}$ \\
\hline
\end{tabular}

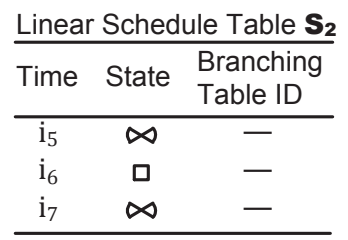

Branching Table $\mathbf{B}_{\mathbf{1}}$

\begin{tabular}{cc}
\hline Condition & $\begin{array}{c}\text { Linear Schedule } \\
\text { Table ID }\end{array}$ \\
\hline Temperature $\leq$ Threshold & $\mathrm{S}_{2}$ \\
Temperature $>$ Threshold & $\mathrm{S}_{3}$ \\
\hline
\end{tabular}

Figure 4.2.2 Schedule and branching tables

Temperature curves when there is time-variant temperature error (a) when both chips are tested with linear schedules $S_{1}$ and $S_{2}$; (b) when by referring to the branching table, $B_{1}$, test of chip $C_{w}$ continues with linear schedule $S_{3}$ after time $i_{4}$. (Curves are only illustrative.) 
are affected by lateral heat dissipation among the cores and also by the limited test bus width which is shared by different cores.

Temperature curves for a $\mathrm{SoC}$ with four cores, as an example, are given in Figure 4.2.3 [He08a]. It shows how the temperatures of the different cores change over time. For a given core, when it is tested, its temperature increases. When a core is not tested, there are no switching activities, and it starts to cool down, as shown by the temperature curve going down.

To guarantee thermal safety, testing is interrupted when a core reaches the high temperature threshold. As shown in Figure 4.2.3, more than one core may be tested at the same time (e.g., the temperature for both core 1 and core 3 is going up around 20us because of testing). Cores will utilize the available TAM which is freed during the cooling intervals of other cores (e.g., core 1 utilizes the cooling time of core 3 at $80 u s$ ) [He08a].

\subsection{Problem Formulation}

The goal is to generate an efficient adaptive test schedule, offline. This is formulated as an optimization problem. The input consists of a SoC design with its set of cores and their corresponding test sequences and their switching activities. The floor plan, the thermal parameters, the static power parameters, and the dynamic power parameters for the chip are given as inputs. The statistical data that models the temperature deviations are also given as input. The adaptive test schedule should be generated to minimize the test application time and the probability of overheating. These objectives are captured by a cost function which expresses the

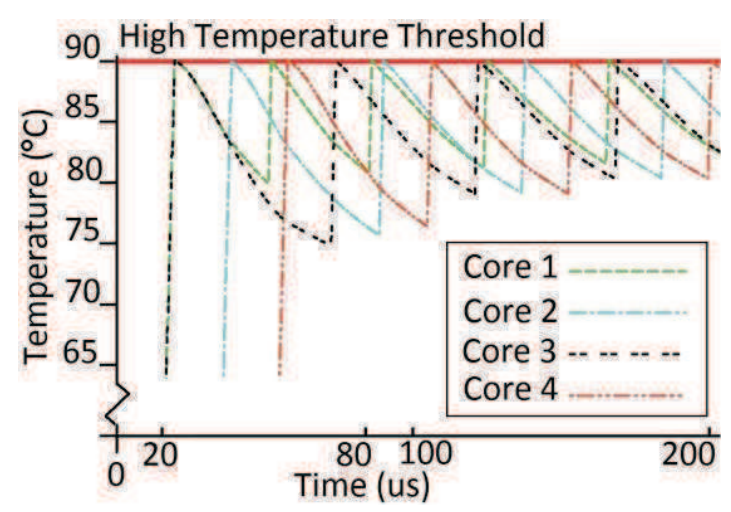

Figure 4.2.3 Temperature curves for a four core chip under test 
overall efficiency of the generated test schedule, as discussed in the following.

The test schedule should be generated under two constraints. The first constraint is the available test bus width. The test bus width limits the number of cores that can be tested in parallel. The second constraint is the available Automatic Test Equipment (ATE) memory which limits the size and the number of the linear schedule tables and branching tables. It is assumed that the available memory after loading the test patterns will be utilized for storing the schedule and, therefore, the amount of memory dedicated to the schedule will not introduce new costs.

In this thesis a comprehensive cost function is defined by combining the cost of the overheated chips and the cost of the test facility operation, as follows:

$$
\text { cost }=\frac{C T F}{T T}+P O C \times \frac{T O P}{(1-T O P)}
$$

The first term in the cost function is related to the test facility operation cost, which is defined as the operational Cost of the Test Facility per time unit $\left(C T F^{1}\right)$ divided by the Test Throughput $(T T)$. The cost of the test facility operation per time unit depends on the cost of the ATE machines, their maintenance costs, and other operational costs. The test throughput captures the applied test size per time unit and is explained later.

The second term of the cost function is related to the cost of the overheated chips, which is the product of the Price of One Chip (POC) and the expected number of overheated chips. The expected number of overheated chips is calculated based on the Test Overheating Probability (TOP) which represents the number of overheated chips per number of chips entering the test facility

In equation 4.3.1 the test overheating probability, TOP, is divided by (1 $T O P$ ) in order to give the expected number of overheated chips per number of non-overheated chips. The cost of the test facility per time unit, CTF, and the price of one chip, $P O C$, depend on the particular manufacturing and test facility and on the particular SoC. To have a simple model for the test throughput, $T T$, assume that the given test facility is characterized by

\footnotetext{
${ }^{1}$ A list of notations and abbreviations is provided in section 4.11.
} 
its overall Effective Test Time per Second (ETTpS) and Test Handling Time (THT).

The effective test time per second is the total test time that the test facility provides. For example if there are two ATE machines working in parallel, the ETTpS could be as high as two. Therefore, the ETTpS depends on the number and specification of the ATE machines and possibly other test facility specifications. The test handling time represents the wasted times that chips are not actually under test (e.g., placing, connecting, and detaching the chips) and therefore, it depends on the test facility specifications. The test throughput, TT, which depends on the Applied Test Size (ATS) and Test Application Time (TAT), is calculated as:

$$
T T=\frac{E T T p S \times A T S}{T A T+T H T} \times(1-T O P)
$$

In order to gain a better understanding of the test throughput, the Normalized Test Throughput (NTT) is defined by normalizing the test throughput, $T T$, to the effective test time per second, ETTpS, and assuming that the test handling time, $T H T$, is negligible, as follows:

$$
N T T=\frac{A T S}{T A T} \times(1-T O P)
$$

The normalized test throughput, NTT, is proportional to the applied test size divided by the test application time. It is also proportional to the percentage of the chips that have completed the test without overheating. Therefore large test application time and large test overheating probability will result in small test throughput and consequently the cost component related to the test facility operation will be higher.

In this thesis, CTF, POC, and ETTPS do not depend on the test schedule and therefore they are considered to be constants. The cost function is then normalized so that all constants are lumped into one new constant, the Balancing Coefficient $(B C)$. The result is the Normalized Cost Function $(N C F)$ which is expressed as:

$$
\begin{aligned}
N C F=\frac{1}{N T T}+B C & \times \frac{T O P}{(1-T O P)}= \\
& =\frac{T A T}{A T S \times(1-T O P)}+B C \times \frac{T O P}{(1-T O P)}
\end{aligned}
$$


The balancing coefficient, $B C$, is in direct proportion to the price of one chip, $P O C$, and in inverse proportion to the cost of the test facility per time unit, CTF. The first term in the above equation captures the test facility operation cost.

The second term captures the balanced cost of the overheated chips and is proportional to the test overheating probability, TOP, and the balancing coefficient. The balancing coefficient balances the cost of the overheated chips against the cost of the test facility operation. Expensive chips will results in a larger balancing coefficient and expensive test facility will result in a smaller balancing coefficient.

\subsection{Temperature Error Model}

As previously defined, temperature error is the difference between the expected temperature (can be estimated by simulation) and the actual temperature (can be measured by sensors). This error can be categorized into spatial temperature error and temporal temperature error. Spatial temperature error shows that different cores have different temperature errors while the temporal temperature error shows that the same core has different errors at different times.

A temperature error model gives the probabilities of the temperature errors for every core in every test cycle. The spatial error model gives the initial error distribution and then the temporal error model is used to recursively estimate the error distribution for the next cycle.

For example, a spatial temperature error model which consists of a discrete distribution shows that at the very beginning of the test the probability of an error equal to $-2.3^{\circ} \mathrm{C}$ in core 1 is 0.001 while the probability for the same error in core 2 is 0.02 . The spatial error model is specified using a look up table which is assumed to be given as one of the inputs. Assuming that the error for a SoC design may range from $-20^{\circ} \mathrm{C}$ to $+20^{\circ} \mathrm{C}$ by a resolution of $0.5^{\circ} \mathrm{C}$, the number of the look up table entries $(M)$ would be 80 for a core and $M \times C$ for a SoC with $C$ cores.

The temporal temperature error model is assumed to be a discrete-time model which means that the temperature error is fixed during a period and then it changes discretely from one period to the next. Therefore, the temporal temperature error model specification has two parts, the period which is called temporal error period and a table of error change 
probabilities. The temporal temperature error table gives the probability of a particular change in error.

For example, a temporal temperature error model shows that the probability that the error increases by $+0.6^{\circ} \mathrm{C}$ is 0.015 . Assume that the temporal error period is $1 \mathrm{~ms}$ and the error is measured to be $-5.3^{\circ} \mathrm{C}$ at time 0 , as shown in Figure 4.4.1. The error will remain $-5.3^{\circ} \mathrm{C}$ up to $1 \mathrm{~ms}(0+$ temporal error period). Then after $1 \mathrm{~ms}$ the exact error is not known any more. However the probability of a certain error can be estimated using the temporal error model. In this example, the probability of a temperature error equal to $\left(-5.3^{\circ} \mathrm{C}+0.6^{\circ} \mathrm{C}\right)=-4.7^{\circ} \mathrm{C}$, between $1 \mathrm{~ms}$ and $2 \mathrm{~ms}$ is 0.015 . Without a measurement at $2 \mathrm{~ms}$, the only available information is that the probability of a temperature error equal to $\left(-4.7^{\circ} \mathrm{C}+0.6^{\circ} \mathrm{C}\right)=$ $-3.1^{\circ} \mathrm{C}$ is $0.015 \times 0.015$, between $2 \mathrm{~ms}$ and $3 \mathrm{~ms}$. In Figure 4.4.1, a new measurement is done at time $3 \mathrm{~ms}$ and the actual error is $-4.7^{\circ} \mathrm{C}$.

The size of the temperature error data set, given as input, might be quite large. In such a case it is necessary to extract a smaller set of data which is representative of the original data in accordance with the accuracy and speed requirements. This is done by clustering the errors into error clusters. The error clusters are characterized by temperature Error-cluster Borders $(E B)$. The temperature error range, resolution, and error clusters are assumed to be identical for all cores, in this thesis.

The Temperature Error Values $\left(T E V_{m}(1 \leq m \leq M)\right)$ and the Spatial Temperature Error Probabilities $\left(\operatorname{STEP}_{c, m}(1 \leq c \leq C ; 1 \leq m \leq M)\right)$ are original inputs which are given for a SoC with $C$ cores for $M$ temperature

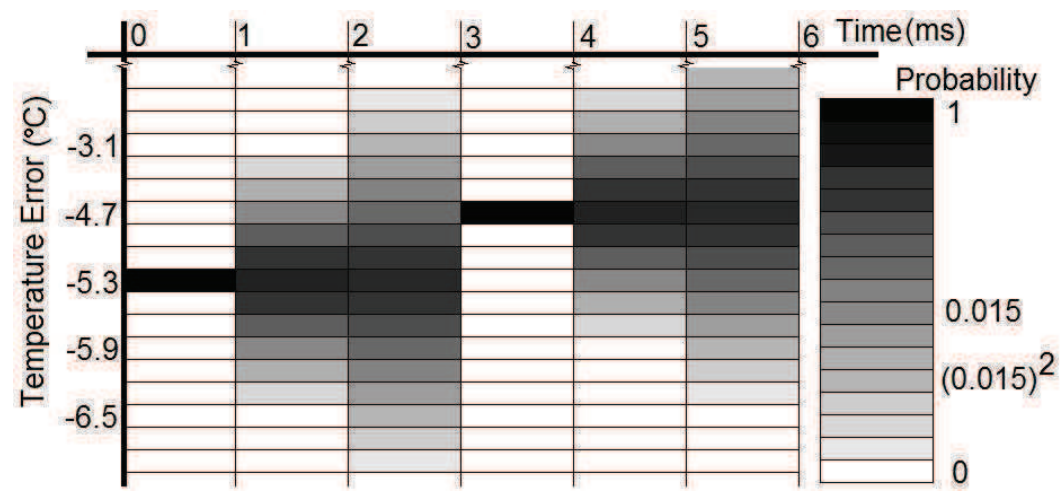

Figure 4.4.1 An example for temporal temperature error probabilities 
error samples. The Temporal Temperature Error Probability (TTEP) is the other input and it gives the probability for a certain change in the error value. The probability that the temperature error value changes from $T E V_{i}$ to $T E V_{j}$ is

$$
P\left(\text { error value change from } T E V_{i} \text { to } T E V_{j}\right)=T T E P\left(T E V_{j}-T E V_{i}\right)
$$

The error clustering is assumed to be uniform and the error-clusters borders, $E B_{l}(0 \leq l \leq L)$, are identical for all cores. Assuming $L$ error clusters, the size of the original data set reduces to $L \times C$. Error clustering will divide the $C$-dimensional error space into error cells indexed using Cartesian system (i.e., $\left.\left[l_{0}, l_{1}, \ldots, l_{C-1}\right]\right)$. For example, assume that for a $\mathrm{SoC}$ with two cores, each core has two error clusters. The 2-dimensional error space is divided into four error cells, indexed with $[0,0],[0,1],[1,0]$, and $[1,1]$. While the original size of the error space is $M^{C}$, the number of error cells is $L^{C}$. Assuming $M=80$ and $C=2$, the original size is $80^{2}=$ 6400 while the size of the clustered error space, with $L=3$, is $3^{2}=9$.

\subsection{Adaptive Test Scheduling}

The proposed adaptive method is based on the on-chip temperature sensors implemented on each core. During test, the actual temperatures of selected cores are read at certain selected moments. A group of chips with similar thermal behavior which are tested with the same schedule is called a chip cluster. During the test, chips are dynamically classified into one of the chip clusters and are tested using its corresponding schedule. The chip clusters vary during the test, and at every adaptation moment (time moment corresponding to a certain branching table) the chip clusters change into a new scheme which is suitable for the new situation.

The parameters that affect the efficiency of the adaptive method are the moments when branching/adaptation happens, the number of edges (i.e., linear schedule tables) and the branching conditions (i.e., chip clustering). For the example in Figure 4.2.2, the adaptation is happening at $i_{4}$, the number of edges is two (two linear schedule tables, $S_{2}$ and $S_{3}$ ), and the branching condition is a comparison with the Threshold temperature.

Since the possible branching moments are multiples of the temporal error period, the first design decision is whether to branch or not at a possible node in a schedule tree. This design decision will be merged with the second design decision which is the number of edges (i.e., the number of 


\section{Chapter 4}

chip clusters). The third design decision is the chip clustering for nodes. These problems are summarized into the following two sub-problems.

1. How many chip clusters, at each possible node in the schedule tree, is suitable? The special case of one edge implies no branching, no sensor reading, and no extra effort.

2. What is the proper chip clustering into the given number of chip clusters? The number of chip clusters is known from the previous question. Depending on the chip clustering some cores may not need sensor readout.

The second question is only relevant when the answer to the first question is larger than one. The above questions are then formulated in two different forms, the first question is described as a tree topology and the second question is the chip clustering for the nodes of that tree topology.

A candidate schedule tree is generated by combining a candidate tree topology with a candidate chip clustering. The number of candidate tree topologies and the number of alternative chip clusterings grow very fast with parameters like temporal error resolution and the number of cores. Since the number of candidate trees is the product of the tree topology alternatives and the chip clustering alternatives, the search space is so huge that ordinary search approaches would not work fast enough. Therefore a constructive method is suggested to deal with this high complexity.

The schedule tree is constructed by adding small partial trees to its leaves. These small partial trees which are the building blocks of the schedule tree are called sub-trees. A sub-tree consists of a small number of linear schedules and branching tables which makes it possible to be clustered and optimized (scheduled) at once. The tree that is under construction with unfinished tests is called an unfinished tree.

For example, assume that there is an unfinished tree, Tree 1, as shown in Figure 4.5.1a. The linear schedule tables of Figure 4.2.2 correspond to the edges of Tree 1 while the branching table corresponds to node 1 , as shown in Figure 4.5.1a. Two sub-trees with one and with two edges are shown in Figure 4.5.1b. Tree 1 has two leaves and combinations of the sub-trees are added to them in order to generate the offspring as shown in Figure 4.5.1c. Offspring 2, for example, is generated by attaching the Sub-tree 1 to node 2 of Tree 1 and attaching the Sub-tree 2 to node 3 of Tree 1 . 
(a) Unfinished Tree

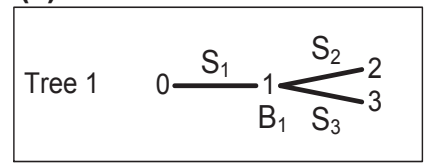

(b) Sub-trees

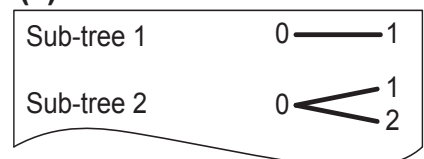

(c) Offspring Trees

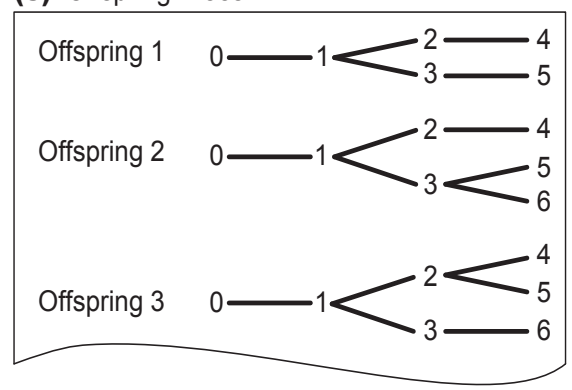

Figure 4.5.1 Constructive method

Main components are (a) Unfinished tree, (b) sub-tree topologies, and (c) offspring trees. For $S_{1}, S_{2}$, $S_{3}$, and $B_{1}$ in (a) refer to Figure 4.2.2.

The proposed constructive algorithm is shown in Figure 4.5.2. The inputs to the algorithm include the switching activities of the tests in order to compute the dynamic power, the thermal error model in order to estimate the temperature errors, and the thermal model of the chip in order to predict the temperatures.

Furthermore, the algorithm requires the electrical model of the chip in order to compute the static power and the dynamic power and in order to be informed about the test bus width limit. The test facility specifications are also inputs to the algorithm which provides the knowledge of the available ATE memory, delay overheads, and the balancing coefficient (i.e., $B C$ in equation 4.3.4).

The algorithm starts with an initialization phase, as shown in Figure 4.5.2. Here, the unfinished tree, sub-tree topologies, temperature error model, and thermal simulator are initialized. Then it proceeds with constructing the schedule tree out of the sub-trees as will be explained in section 4.5.1. The linear schedule tables are discussed in section 4.5.2. The sub-tree evaluation is explained in section 4.5.3. The sub-tree scheduling which is based on an optimization heuristic is explained in section 4.5.4.

\subsubsection{Tree Construction}

The schedule tree construction starts with a root node and in each iteration an unfinished tree extends and multiplies by adding alternative combinations of sub-trees to its active leaf nodes, as shown in Figure 4.5.1. Then, a small number of promising under-construction trees are selected as unfinished trees from the offspring list to be used in the next iteration. 


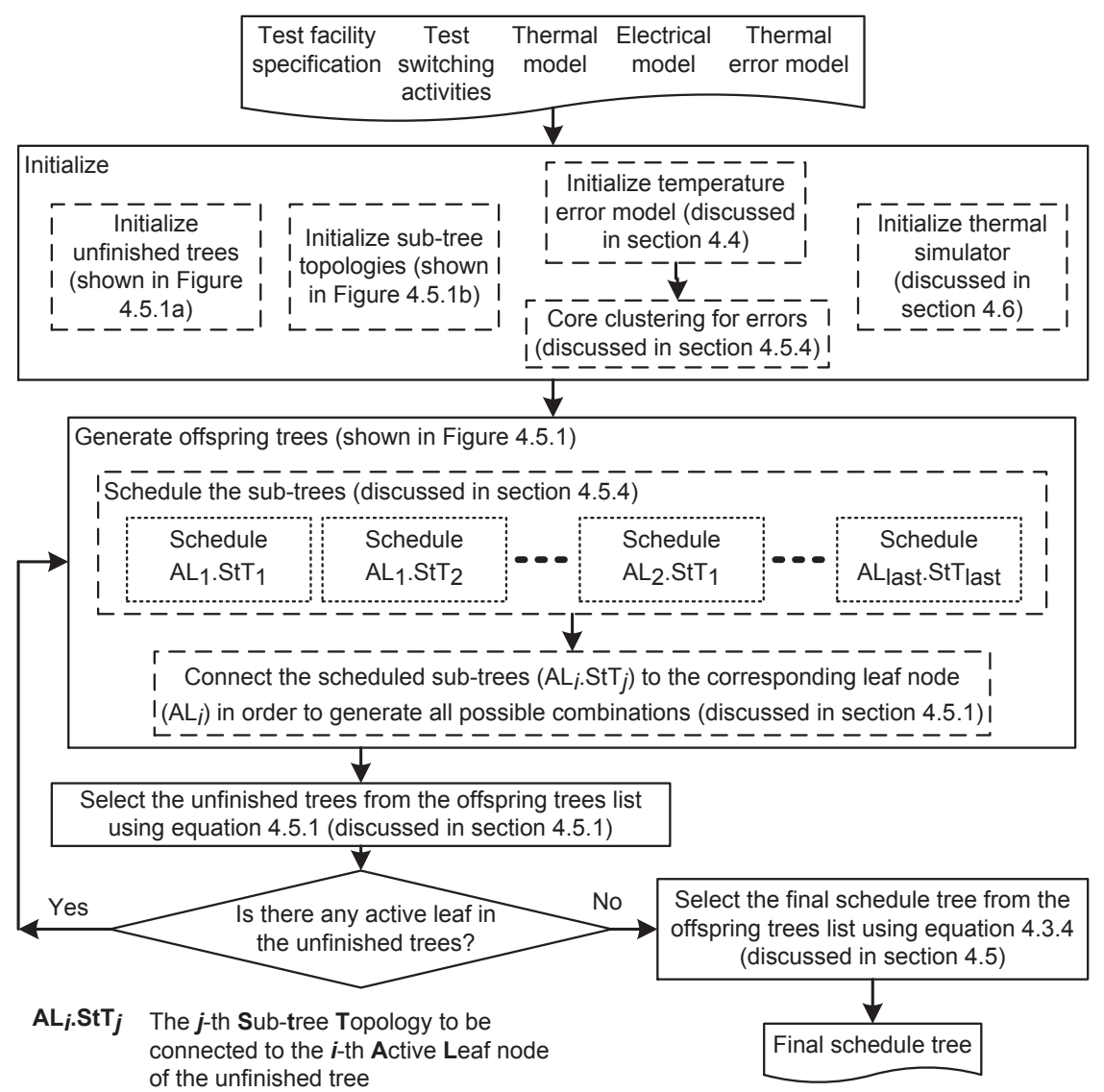

Figure 4.5.2 The proposed constructive method

For example, an unfinished tree list will be selected from the offspring list (partially shown in Figure 4.5.1c) to go on with. The algorithm, as shown in Figure 4.5.2, ends when all the unfinished trees have completed the test.

The selection process keeps the ATE memory constraint satisfied by not selecting the candidates that will exceed the memory limit. A naïve algorithm will have a tendency to create many edges in all iterations at the beginning since it reduces the cost. As a result of this naïve approach the algorithm will put many edges near the root of the tree and later on as the memory fills up there will not be any possibility to add a new edge. In order to provide the algorithm with the freedom to put more edges in the more beneficial regions. In our proposed algorithm, the selection is done based on the Scaled Cost Function (SCF) as defined in the following. 


$$
S C F=N C F \times(\text { adjusting_offset }+ \text { number_of_nodes })
$$

The normalized cost function, NCF (equation 4.3.4), is scaled by the tree's number of nodes plus an adjusting offset. Now, adding nodes to the tree is only beneficial if it gives a reasonable cost reduction, otherwise a smaller tree may get a lower scaled cost and manage to survive to the next iteration, while bigger trees are discarded. In general bigger trees will have smaller $N C F$ but not necessarily smaller $S C F$. The effect of the number of nodes is adjusted by the adjusting offset. A small adjusting offset promotes having fewer edges compared to a large adjusting offset which promotes having more edges. In other words, a larger adjusting offset reduces the sensitivity to the number of nodes. An extremely large adjusting offset means that the number of nodes has almost no effect on decision making while NCF dominates the decision making process.

To satisfy the memory constraint, when unfinished tree is selected based on its scaled cost function, it is scheduled for the rest of test by just using the linear schedule tables which mean no further branching. During this scheduling, the linear scheduling aborts as soon as the memory limit is violated. If the linear scheduling succeeds in respecting the memory limit, the candidate survives to the next iteration. Otherwise, the currently chosen unfinished tree is discarded and the next candidate with larger or equal scaled cost is tested for its compliance with the memory constraint. The scheduling will fail if no candidate could meet the memory constraint, meaning that the limit is too tight even for a linear schedule.

\subsubsection{Linear Schedule Tables}

A linear schedule table captures a schedule without branching. The linear schedule table entries (start/stop times for each and all cores) are optimized in the offline phase to reduce the probability of overheating. The temperatures are checked frequently in order to keep the overheating probability small.

The start/stop states in the linear schedule tables are generated using the heuristic proposed in [He08a]. According to this heuristic, the test of the cores with lower temperature and higher remaining test size will be started or resumed earlier. Activating the cores with lower temperatures is desirable because it provides longer testing intervals and therefore reduces the number of test partitions and their corresponding overheads. 


\section{Chapter 4}

Moreover, by choosing the colder cores while the effect of adjacent cores are taken into account by temperature simulation, in fact, the algorithm activates the cores which are far from the current active cores. This will save the newly activated cores from the accumulated heat in their possible neighbors and furthermore by not activating the adjacent cores, the newly deactivated cores will experience a faster cooling. The heuristic gives also advantage to the cores with longer remaining tests, thus maximizing the interleaving opportunities. Besides, the situation in which a long test sequence leads to a long total test application time is avoided.

As mentioned before, each chip cluster is tested with a dedicated linear schedule. Every chip cluster is represented by a single error value which will be used to estimate the actual temperature based on the simulated temperature; this error value is called representative temperature error. The estimated temperature is updated periodically by correcting the cores' simulated temperatures with the representative temperature error. The estimated temperature is then used to compute the static power and to determine the 'state' of the cores (i.e., testing or cooling).

For example, assume that there are two chips $\left\{D_{0}, D_{1}\right\}$ in a certain chip cluster and the chips consist of only one core. Therefore, at a certain moment in time, there are two error values $\left\{E_{0}, E_{1}\right\}$ corresponding to the two chips. But the linear scheduling heuristic works with one error value for one chip cluster. Therefore, the representative temperature error, $r$, which is a real number $(r \in \mathbb{R})$ is defined as a value which represents chips error values, $\left\{E_{0}, E_{1}\right\}$.

The representative temperature error is updated periodically with the temporal error period (see section 4.4) while the estimated temperature, static power, and state of the cores are updated more frequently. After updating the state of the cores, the dynamic power sequence is computed. The initial temperatures are available as the results of the previous temperature simulation. Having dynamic and static power sequences in addition to the initial temperatures, the next temperature simulation is performed.

The representative temperature error for a chip cluster is viewed as a safety margin in [Aghaee10] and its optimal value is experimentally computed for a number of examples. Those experiments suggest that the optimal value for a representative temperature error is equal to the border between the chip cluster and the adjacent chip cluster that has larger error (i.e., 
hottest possible chip in the chip cluster). This is true for all chip clusters except the last one that has the largest error. For example, for a chip cluster stretching from $E B_{i}$ to $E B_{j}\left(E B_{i}<E B_{j} ; j<L\right), E B_{j}$ would be a good choice to be the representative temperature error for this chip cluster. The representative temperature errors are assigned in a similar way in this thesis.

To have an example from a different point of view, assume that in total there are four chips $\left\{D_{0}, D_{1}, D_{2}, D_{3}\right\}$ and chips consist of only one core. Therefore, at a certain moment in time, there are four error values $\left\{E_{0}, E_{1}, E_{2}, E_{3}\right\}$ corresponding to the four chips. Assume that $E_{0}<E_{1}<$ $E_{2}<E_{3}$. Assume that the chip-clustering algorithm (will be explained in section 4.5.4) has generated two chip clusters $\left\{D_{0}, D_{1}\right\}$ and $\left\{D_{2}, D_{3}\right\}$. The representative temperature error for the chip cluster that has smaller errors (i.e., $\left.\left\{D_{0}, D_{1}\right\}\right)$ is $r^{0}=E_{1}$ and the representative temperature error for the last chip cluster, $r^{1}$, is formulated as an optimization variable along with the chip-clusters borders in the chip-clustering algorithm. This is in particular important when the number of chip clusters is small. This is usually the case ${ }^{2}$ and therefore the cluster on the high temperature extreme will contain a non-negligible number of chips.

The sub-tree optimization method encodes the problem based on chipclusters borders. The representative temperature errors are defined as chipclusters borders for all chip clusters but the last one. For the last error cluster (one with the largest errors), the representative temperature errors are encoded along with the chip-clusters borders as the sub-tree optimization variables. This will be explained in more details in section 4.5.4.

The optimization problem for a linear schedule table is to minimize the partial normalized cost function by finding the proper start/stop times. This is done based on the heuristic proposed in [He08a]. The utilized test bus width is the sum of the TAM widths of the active cores for tests which utilize the TAM. The schedule size is the product of the number of the linear schedule table entries and the record size. The schedule tree is equivalent to a number of linear schedule tables (edges) in addition to a number of branching tables (nodes), as shown in Figure 4.5.1a. The linear

${ }^{2}$ Refer to [Aghaee10]. 


\section{Chapter 4}

schedule table is explained above and the rest of the construction process will be explained in the following sections.

\subsubsection{Sub-Tree Evaluation}

The schedule tree is constructed by attaching sub-trees to the leaves of the unfinished trees (See Figure 4.5.1). For this purpose, the proper schedule for a sub-tree topology should be found. In a sense, a sub-tree is a tree and the cost function introduced in section 4.3 should be usable. However, there is a subtle difference between their objectives. For the schedule tree the objective is its very own cost. For a sub-tree the objective is, on the contrary, the cost of the schedule tree that is to be constructed. Therefore, the cost of the final schedule tree should be estimated assuming that this particular sub-tree is used in its construction. This makes the cost evaluation different for the sub-trees.

To find the near optimal schedule for a sub-tree topology, a partial cost function must be used for different sub-tree clustering alternatives. For the evaluation of the cost function (i.e., NCF in equation 4.3.4), the expected values of the test application time, $T A T$, the applied test size, $A T S$, and the test overheating probability, TOP, (denoted by ETAT, EATS, and ETOP, respectively) should be computed by utilizing the temperature error statistics.

The expected values are computed while each edge is being scheduled. In the formulation of the schedule tree, an edge is represented by its destination node. Assuming that the number of nodes is $N$, the Nodes' Probabilities $\left(N P_{n}(1 \leq n \leq N)\right)$, the Nodes' Applied Test Sizes $\left(\operatorname{NATS}_{n}(1 \leq n \leq N)\right)$, and the Nodes' Test Application Times $\left(\operatorname{NTAT}_{n}(1 \leq\right.$ $n \leq N)$ ) are used to compute the expected applied test size and the expected test application time as follows:

$$
\begin{aligned}
& E A T S=\sum_{n=1}^{N}\left(N A T S_{n} \times N P_{n}\right) \\
& E T A T=\sum_{n=1}^{N}\left(N T A T_{n} \times N P_{n}\right)
\end{aligned}
$$

In order to explain the expected test overheating probability, ETOP, and understand how node probabilities are computed, the notion of node clustering and error cells are introduced here. Temperature errors of cores constitute a $C$-dimensional errors space ( $C$ is the number of cores). For example in Figure 4.5.3, there are two cores and therefore the error space is two dimensional. The horizontal axis represents the error values of the 
first core and the vertical axis represents the error values of the second core. There are four error clusters for each core and therefore there are sixteen error-cells in Figure 4.5.3.

This is specifically important for the nodes at which branching takes place. Branching at a node is, in fact, a chip clustering to a number of groups, so that each chip cluster corresponds to an exclusive edge that branches out of that node. Chips are identified by their cores' errors and therefore a chip clustering is a partitioning of the $C$-dimensional error space into a number of chip clusters.

This means that a chip cluster is a combination of specific error intervals of the cores. A candidate 'sub-tree clustering' is a set of chip clustering alternatives for nodes. Furthermore, a candidate 'sub-tree clustering' could be viewed as a set of nodes' clustering alternatives for a sub-tree topology. An error cell is a cell in $C$-dimensional error space separated by cores' error-clusters borders and therefore its projection on a core error axis is an error cluster for that core. Therefore, a node clustering could be seen as assigning error cells to chip clusters or equivalently labeling error cells with chip clusters. An example for labeling of the error cells is shown in Figure 4.5.3. There are two cores $(C=2)$ in the figure, and the numbers (" 0 " or " 1 " in this case) inside the rectangular error cells are the labels.

A candidate sub-tree topology will have a number of candidate clustering alternatives which label the nodes' error cells with the relevant chip clusters. Each chip cluster for a node corresponds to an edge branching out of that node and corresponds to a linear schedule table. Each node has its own dedicated Error-Cell Labeling $\left(E C L_{n, l_{1}, l_{2}, \ldots, l_{C}}\left(1 \leq n \leq N ; 1 \leq l_{i} \leq\right.\right.$ $L)$ ). Looking from a branching node, a succeeding node corresponds to a

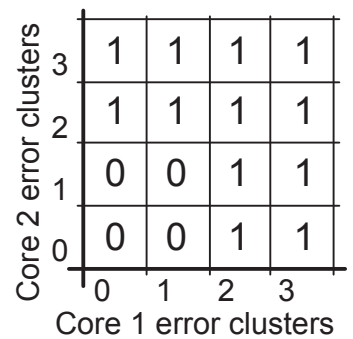

Figure 4.5.3 An example for error-cells labeling

Four error-cells are labeled with 0 that is the ID of the chip cluster number 0 and the remaining twelve error-cells are labeled with 1 that is the ID of the chip cluster number 1. 
chip cluster and therefore it receives a Node's Cluster Label $\left(N C L_{n}(1 \leq\right.$ $n \leq N$ )) to represent that chip cluster (or equivalently the preceding edge and corresponding linear schedule). This label indicates which of the branching node's chip clusters will lead to a certain succeeding node.

The probabilities of error cells for different nodes and consequently the probabilities of those nodes are computed based on the temperature error model and based on the chip clusterings of the preceding nodes. In order to speed up the computation of the Error-Cells Probabilities (ECP) the Error Cell Change Probabilities (ECCP) are pre-computed as shown below, in equation 4.5.5. The error cell change probabilities are, in fact, the concentrated effect of the temporal error model which is repeatedly used to compute the error-cells and nodes probabilities.

It is assumed that the variation in the probabilities inside an error cluster is negligible. Furthermore, it is assumed that the error change probabilities for different cores are independent. The error-cell probabilities change from node to node and therefore most of the time the equations are about two nodes, the origin and the destination. The error cells for the origin node are superscripted with $O$ and for the destination node with D. ECCP is computed as follows:

$$
\begin{aligned}
& E C C P_{l_{1}^{O}, l_{2}^{O}, \ldots, l_{C}^{O}, l_{1}^{D}, l_{2}^{D}, \ldots, l_{C}^{D}}^{\text {befor normalion }}= \\
& \quad \prod_{c=1}^{\mathrm{C}} \sum_{i=E B\left(l_{c}^{O}\right)}^{E B\left(l_{c}^{O}\right)} \sum_{j=E B\left(l_{c}^{D}-1\right)}^{E B\left(l_{c}^{D}\right)} \operatorname{TTEP}\left(T E V_{j}-T E V_{i}\right)
\end{aligned}
$$

TTEP is the temporal temperature error probability, TEV is temperature error value, and $E B$ is error cluster border. $E C C P$ is computed as follows:

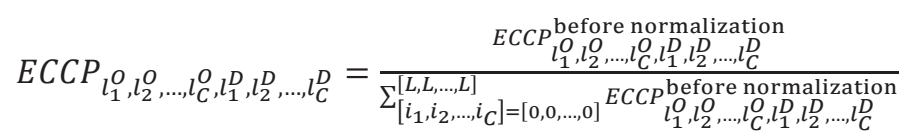

The error-cell probabilities for the root node (i.e., $n=0$ ) are computed based on the spatial temperature error probabilities (STEP) as follows:

$$
E C P_{n, l_{1}, l_{2}, \ldots, l_{C}}=\prod_{c=1}^{C} \sum_{i=E B\left(l_{c}-1\right)}^{E B\left(l_{c}\right)} S T E P_{c, i} ; \text { for } n=0
$$

The error-cell probabilities for non-root nodes (i.e., $n>0$ ) are computed based on the predecessor node which is denoted by $p n$. First, error-cell probabilities just after the branching are extracted from the predecessor node as follows: 
$E C P_{n, l_{1}, l_{2}, \ldots, l_{C}}^{\text {after branching }}=\left\{\begin{array}{cc}E C P_{p n, l_{1}, l_{2}, \ldots, l_{C}} & ; \text { if } E C L_{p n, l_{1}, l_{2}, \ldots, l_{C}}=N C L_{n} \\ 0 & ; \text { otherwise }\end{array}\right.$

While scheduling an edge, overheating may occur to some of the cells (ranges of chips) which have larger temperature errors. Consequently, the probability of these cells at the end of the edge (after the corresponding chunk of the test is applied) is considered to be zero. The error-cell probabilities, ECP, after overheating are computed based on Representative Temperature Error (RTE) (RTE is introduced in section 4.5.2) as represented below, in equation 4.5.8. Overheating of a core occurs when the core's actual temperature which is estimated by adding RTE to the Simulated Temperature (ST) exceeds the High Temperature Threshold (HTT). A chip is considered as being overheated if at least one of its cores overheat.

$E C P_{n, l_{1}, l_{2}, \ldots, l_{C}}^{\text {after overhing }}=\left\{\begin{array}{cc}E C P_{n, l_{1}, l_{2}, \ldots, l_{C}}^{\text {after branching }} & ; \text { if } \forall c\left(R T E_{n}^{c}+S T_{n}^{c}\right)<H T T \\ 0 & ; \text { otherwise }\end{array}\right.$

According to the temperature error models (introduced in section 4.4) the error-cell probabilities, ECP, after temporal changes are computed as:

$E C P_{n, l_{1}^{D}, l_{2}^{D}, \ldots, l_{C}^{D}}^{\text {after temporal changes }}=$

$$
=\sum_{\left[l_{1}, l_{2}, \ldots, l_{C}\right]=[0,0, \ldots, 0]}^{[L, L, L]} E_{n, l_{1}, l_{2}, \ldots, l_{C}}^{\text {after overheating }} \times \operatorname{ECCP}_{l_{1}, l_{2}, \ldots, l_{C}, l_{1}^{D}, l_{2}^{D}, \ldots, l_{C}^{D}}
$$

The node's probability, $N P$, is computed as follows:

$$
N P_{n}=\sum_{\left[l_{1}, l_{2}, \ldots, l_{C}\right]=[0,0, \ldots, 0]}^{[L, \ldots, L]} E_{n, l_{1}, l_{2}, \ldots, l_{C}}^{\text {after branching }}
$$

Node's not Overheating Probability $(\mathrm{NnOP})$ is the probability that a chip which corresponds to this edge according to the chip clustering scheme, is not overheated after traversing this edge. $N n O P$ for a node, $n$, is computed as follows:

$$
N n O P_{n}=\frac{\sum_{\left[l_{1}, l_{2}, \ldots, l_{C}\right]=[0,0, \ldots, 0]}^{\left[L, \ldots P_{n, l_{1}, l_{2}, \ldots, l_{C}}\right.}}{N P_{n}}
$$

Finally, error-cell probabilities, ECP, are computed as:

$$
E C P_{n, l_{1}, l_{2}, \ldots, l_{C}}=\frac{E C P_{n, l_{1}, l_{2}, \ldots, l_{C}}^{\text {after temporal changes }}}{N P_{n}} \text {; for } n>0
$$


Edges are scheduled by determining the linear schedule tables as explained in section 4.5.2. Then the candidate sub-tree clustering is evaluated using the partial cost function which is based on the expected applied test size, the expected test application time, and the predicted test overheating probability. The first two are already introduced in equation 4.5.2-3 and the last one is explained below.

Evaluation of a partial tree is in fact an attempt to predict the cost of the completed schedule tree, based on the current situation of that partial tree. For this purpose, it is assumed that the final schedule tree will be composed of a number of similar partial trees (building blocks for the final schedule tree). These partial trees are assumed to have similar expected applied test size, expected test application time, and expected test overheating probability. These expected values are assumed to be similar to those of the partial tree that we are evaluating.

Therefore, a good prediction approach for the test application time and the applied test size would be their current expected values multiplied by the predicted total number of partial trees. Since only the ratio of the predicted test application time to the predicted applied test size matters in the cost function (the first term in equation 4.3.4), a good choice for predicted values of these variables is their expected values. But the situation for Predicted Test Overheating Probability (PTOP) is different since its value does not change linearly when a number of similar partial trees (building blocks) are put one after the other (unlike EATS and ETAT).

Assuming that there are $Q$ leaves in the tree, the Leaf's Overheating Probability $\left(\operatorname{LOP}_{q}(1 \leq q \leq Q)\right)$ is the overheating probability for the path from the root node to the specified leaf node. Its computation includes multiplication over nodes that belong to the specified root-to-leaf path. The overheating probability for leaf $q$ is computed as:

$$
L O P_{q}=1-\prod_{n} \mathrm{NnOP}_{n}
$$

$q$ is a leaf node for all nodes, $n$, belonging to the root-to- $q$ path

The expected test overheating, assuming a total of $Q$ leaf nodes, is computed as:

$$
E T O P=\sum_{q=1}^{Q}\left(L O P_{q} \times N P_{q}\right)
$$


ETOP can be used in equation 4.3.4 to evaluate a fully constructed schedule tree, but for partial cost function when the tree is not yet fully constructed, the predicted test overheating probability, $P T O P$, is used in to evaluate the partial cost function (which will replace $N C F$ in equation 4.5.1). PTOP is computed as:

$$
P T O P=1-(1-E T O P)^{\lambda}
$$

Where $\lambda$ is the total number of partial trees (building blocks) that are assumed to be similar to the current partial tree and will construct the final schedule tree. ETOP is computed for the partial tree as expressed in equation 4.5.14 and then the predicted test overheating probability is computed by assuming that these $\lambda$ partial trees have overheating rates equal to the current partial tree's overheating rate. $\lambda$ is computed based on the expected Number of Partial Trees (NPT) which is defined as the total test size divided by the expected applied test size, EATS, for the current partial tree.

A naïve algorithm will use the NPT instead of $\lambda$ in equation 4.5.15. However, because of the localities in the schedule tree, partial trees (building blocks) with a lot of cooling may exist. For these partial trees, the expected applied test size is small and consequently the expected number of partial trees, NPT, will be estimated pessimistically. This unrealistic estimation may result in exceedingly large predicted test overheating probabilities, PTOP, and consequently a long schedule tree with too much of cooling may receive a low cost and be selected.

Therefore, limiting the expected number of partial trees, NPT, would be helpful for good schedules to receive a more realistic cost. A reciprocal limiter is used here which amplifies small inputs and attenuates large inputs. In the proposed reciprocal limiter, the output is always one when the input is one and the output is equal to the input in a point that is called Knee. The output will be always smaller than the limit which is (Knee + 1). The limited output, $\lambda$, is calculated based on the input, NPT, as follows:

$$
\lambda=(\text { Knee }+1)-\frac{\text { Knee }}{N P T}
$$

A larger Knee promotes lower overheating since the maximal value of $\lambda$ increases and also because of the increased limiter's amplification for the NPT values which are less than Knee. On the other hand, a smaller Knee will result in schedules with shorter test application time. 


\section{Chapter 4}

At this point, the introduction to computation of the expected test application time, expected applied test size, expected test overheating probability, and predicted test overheating probability is completed and the expected cost for a sub-tree could be computed using them. Therefore, the clustering alternatives for a sub-tree topology could be evaluated using the scaled cost (equation 4.5.1). The clustering alternatives are explored by PSO and the best scheduled sub-tree is selected at the end. This optimization is further discussed in the next section.

\subsubsection{Sub-Tree Scheduling}

As mentioned before, the schedule tree is constructed by attaching subtrees to unfinished trees' leaves (See Figure 4.5.1). For this purpose, the proper schedule for a sub-tree topology should be found. In order to schedule a sub-tree topology which is going to be connected to the specified leaf node of the unfinished tree $\left(\mathrm{AL}_{i}\right.$. StT $\mathrm{T}_{j}$ in Figure 4.5.2) a heuristic, as shown in Figure 4.5.4, iteratively generates alternative chip clustering schemes and evaluates them. The evaluation is explained in section 4.5.3 and requires the sub-trees' edges (i.e., linear schedule tables) to be scheduled as explained in section 4.5.2.

A chip clustering scheme for a sub-tree specifies which chips will take which edges. The chips are specified by their cores' errors and therefore the problem could be seen as assigning chip clusters to the error cells located in the $C$-dimensional error space. The search space could be seen as the collection of different alternatives for $E C L_{n, l_{1}, l_{2}, \ldots, l_{C}}$. For example for a chip with two cores, the general form is $E C L_{n, l_{1}, l_{2}}$ and therefore, for a sub-tree with two nodes, the solutions will be similar to the two alternatives given in Figure 4.5.5.

A solution encoding scheme is suggested in [Aghaee11b] which labels the error cells with chip clusters. The number of the decision variables grows exponentially with the number of cores and therefore the computational complexity is very high. In this thesis, we suggest a solution encoding scheme which encodes the chip-cluster borders instead of the error cells.

For a node with $S$ succeeding chip clusters the number of decision variables is $S \times C$. For $S$ chip clusters, there are $(S-1)$ chip-cluster borders and the $S$-th value is the representative temperature error for the last cluster. Here, the number of the decision variables grows in proportion to the number of 


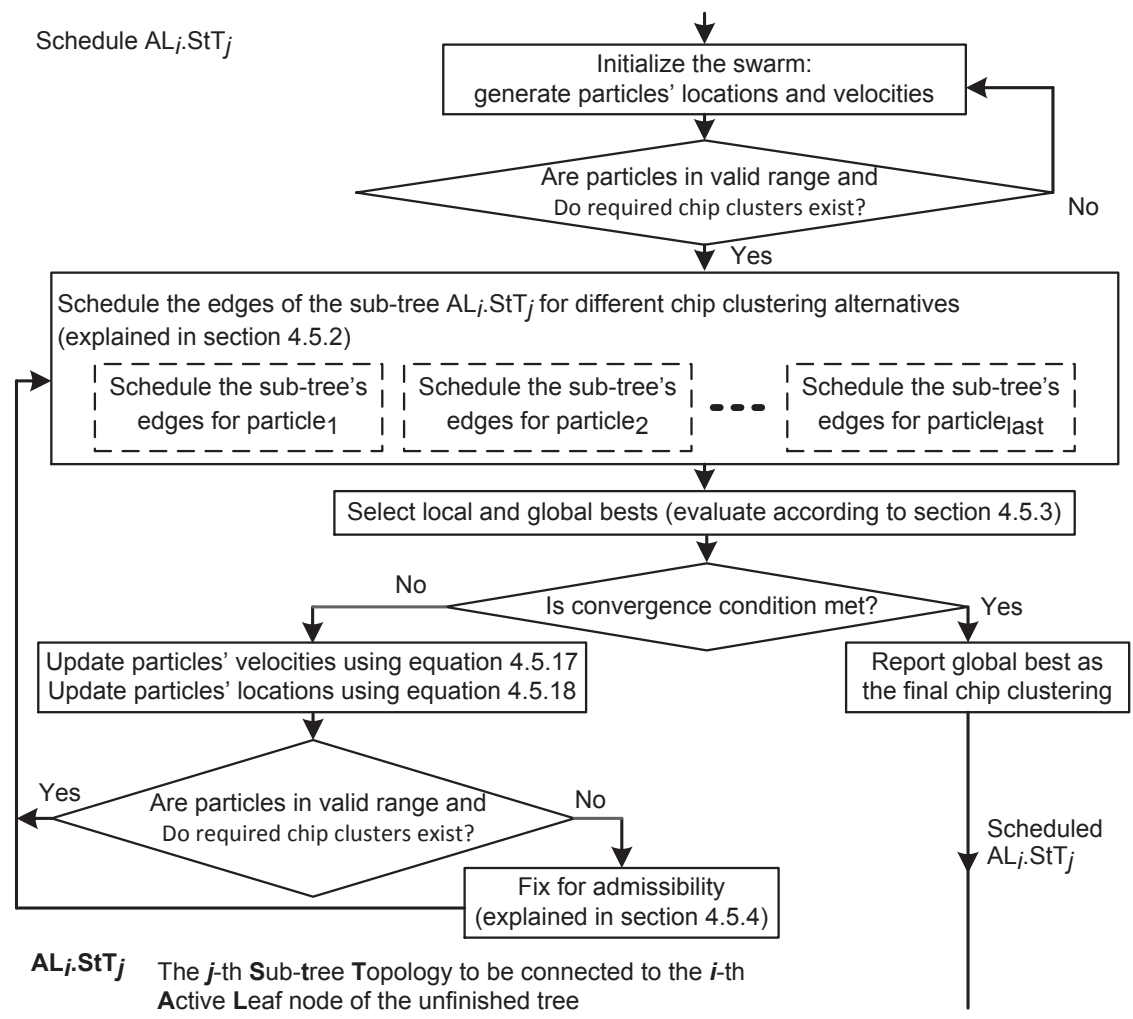

Figure 4.5.4 Sub-tree optimization algorithm

cores and therefore the computational complexity is much smaller compared with the scheme suggested in [Aghaee11b].

Two examples for the suggested solution encoding for a sub-tree with only one node, similar to sub-tree 2 in Figure 4.5.1b, are shown in Figure 4.5.6. The solutions correspond to a SoC which has only two cores. There are three temperature error clusters per core and the number of edges (i.e., number of chip clusters) in the corresponding sub-tree is two. $r_{1}$ and $r_{2}$ are representative temperature errors for the last chip cluster. The 0 -th chip cluster in Figure 4.5.6a is larger than the 0-th chip cluster in Figure 4.5.6b. An equivalent view-point is to compare the number of error cells which are indexed by 0 . Another equivalent view-point is to compare the chipclusters borders on the vertical axes (i.e., third element in the solutions encodings). 
The Labeling Plan

\begin{tabular}{|l|l|l|l|}
\hline$E C L_{0,0,0}$ & $E C L_{0,0,1}$ & $E C L_{0,1,0}$ & $E C L_{0,1,1}$ \\
\hline$E C L_{1,0,0}$ & $E C L_{1,0,1}$ & $E C L_{1,1,0}$ & $E C L_{1,1,1}$ \\
\hline
\end{tabular}

Alternative 1

\begin{tabular}{|l|l|l|l|}
\hline 0 & 0 & 1 & 1 \\
\hline 1 & 1 & 1 & 0 \\
\hline
\end{tabular}

Alternative 2

\begin{tabular}{|l|l|l|l|}
\hline 0 & 0 & 0 & 1 \\
\hline 0 & 1 & 1 & 1 \\
\hline
\end{tabular}

Figure 4.5.5 Error-cell labeling alternatives

Two alternatives are for a chip with two cores and a sub-tree with two nodes. The general form is $\boldsymbol{E} \boldsymbol{C} \boldsymbol{L}_{n, \boldsymbol{l}_{1}, \boldsymbol{l}_{2}}, \boldsymbol{n}$ being the node index (row). Cells (columns) are indexed by $\boldsymbol{l}_{\mathbf{1}}$ and $\boldsymbol{l}_{\mathbf{2}}$.

The possible solutions are then explored using particle swarm optimization. Although PSO is introduced in section 2.7, let us briefly review it here. A candidate solution is called a particle and is represented by its location and its velocity. The locations are the encoded solutions and the velocities are used to determine the next candidate solutions. Each particle remembers its previous best location, and the swarm remembers the global best solution that is the best location any of its particles have visited ever. The previous bests and the global best are then used to give a hint to the random velocities.

(a)

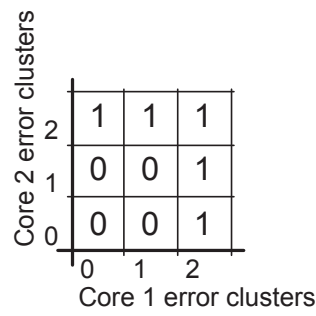

Solution encoding is $\left[1, r_{1}, 1, r_{2}\right]$ (b)

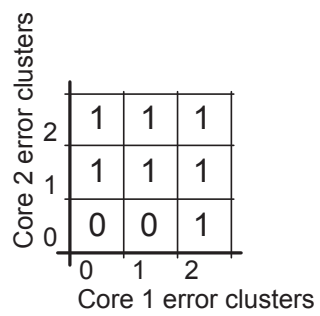

Solution encoding is $\left[1, r_{1}, 0, r_{2}\right]$

Figure 4.5.6 Two examples for error-cells labeling

Error cells are labeled with chip clusters' IDs (numbers inside the small rectangles). The solution encodings are given below the error spaces. 
A canonical form of PSO uses equation 4.5.17, below, to update the velocities. The coefficients in equation 4.5.17 are given as a part of the chosen canonical form [Poli07]. random ${ }_{0}$ and random $_{1}$ are two distinct randomly generated numbers between 0 and 1 . The location and the velocity on the right hand side of equation 4.5.17 are the current values, and the left hand side velocity is the next value.

$$
\begin{aligned}
& \text { velocity }=0.7298 \times \text { vvelocity }++\left[2.05 \times \text { random }_{0} \times(\text { previousBest }- \text { location })\right]+ \\
& \left.+\left[2.05 \times \text { random }_{1} \times(\text { globalBest }- \text { location })\right]\right\}
\end{aligned}
$$

Since the location, in this sub-tree scheduling problem, is a natural number, the next location is the rounded sum of the current location and the next velocity, as expressed in equation 4.5.18, below:

location $=$ Round $($ location + velocity $)$

There are two admissibility conditions to ensure that the particles are valid solutions. The first condition is the valid range and the other is the presence of required chip clusters. For example assume that the errors range from -10 to +10 and therefore smaller or larger errors will never happen in practice. If it happens that one element in the next particle's location is +11 , then this particle is out of range.

An example for a required chip cluster not being present is as follows. Assume that there are three edges in a certain node and, therefore, three chip clusters are necessary. It may happen that in the next particle's location, the first and the second chip-cluster borders are assigned with identical values and therefore the second chip cluster is missing.

The proposed solution encoding which is based on chip-clusters borders works well with particle swarm optimization, since the location and velocity in PSO's terminology correspond to the location and velocity for chip-clusters borders. A typical particle in the beginning is far from being good and experiences a high velocity towards the better location since typically the difference between the best location and the current location is large at the beginning. Therefore a rapid convergence towards the preferred value for the chip cluster border will take place.

Later on, a typical particle will be close to the optimal location and according to equation 4.5.17 it will move slower, thus pinpointing the 
preferred value for the chip cluster border. Some experiments, for chip clustering optimization for sub-trees using PSO, are reported in [Aghaee11b]. The experiments showed that the PSO performs well for this purpose. Therefore, it is used here as a part of the proposed SoC test scheduling technique.

\subsubsection{Remarks}

The proposed optimization technique is structured so that it enables parallel implementations with different granularities. The alternative subtree topologies $\left(\mathrm{AL}_{i} . \mathrm{StT}_{j}\right.$ in Figure 4.5.2) could be optimized in parallel. For example, assuming one unfinished tree with two leaf nodes and three sub-tree topologies, there will be $3^{2}=9$ combinations to optimize in parallel.

Furthermore, at the lower level of sub-tree scheduling, each alternative chip clustering in PSO (particle ${ }_{i}$ in Figure 4.5.4) could be generated (corresponding edges being scheduled) in parallel with other alternative schedules. The scheduling of the edges (i.e., optimizing the linear schedule tables) is the part that requires temperature simulation (dashed-line blocks in Figure 4.5.4). Therefore, these computationally expensive parts could be implemented in parallel in two different nested levels.

The proposed adaptive approach in this thesis combines the benefits of an online scheduling technique with the benefits of an offline scheduling approach and avoids their shortcomings. An online schedule will introduce very large overheads that are associated with sensor readouts, decision making process, and pausing/resuming the tests. An offline schedule, on the other hand, is not capable of reacting to variations but has no run-time overheads. In a fully online approach, reading the temperature sensors for all cores as often as it is necessary and making the corresponding decisions based on the acquired data will cause a very large load on the test access mechanism and will introduce large delays to the schedule. Our proposed approach uses temperature simulations as much as possible offline and accesses carefully-selected cores' sensors at carefully-selected times during the test.

There is one schedule tree for a chip that addresses all cores individually. For example, in a linear schedule table that corresponds to an edge, it is stated that at time $t_{1}$ cores $C_{0}$ and $C_{2}$ are being tested, while cores $C_{1}$ and $C_{3}$ are cooling. It might be that at another time, $t_{5}$, cores $C_{1}$ and $C_{2}$ are 
being tested, while cores $C_{0}$ and $C_{3}$ are cooling. The linear schedule table is similar to $S_{1}$ in Figure 4.2.2, but instead of the second column that shows only one column for state (in Figure 4.2.2), there are as many state columns as there are cores. There is only one branching table for one node in the schedule tree (similar to $B_{1}$ in Figure 4.2.2) but it contains, in every row, conditions that include at least one core and at most all the cores.

\subsection{A Fast Temperature Simulation Approach}

In order to evaluate the candidates, the test application time and the test overheating probability should be computed as previously explained. In order to calculate the test application time and the test overheating probability, the temperatures of the cores are required. Therefore, for every candidate schedule which is examined by the meta-heuristic (particle ${ }_{i}$ in Figure 4.5.4), temperature simulation should be performed. Temperature simulation is in the main loop in Figure 4.5.4 which itself is in the main loop in Figure 4.5.2. This means that the temperature simulation which is performed inside the optimization loop is repeated numerously.

On the other hand, the temperature simulation is the slowest step in the iterative part of the algorithm. Therefore, the temperature simulation is the bottleneck. It limits the number of the cores which can be handled by the proposed method. Moreover, it is, also, a limiting factor for the quality of the schedules.

Since the optimization heuristic will have a time consuming process inside its main loop, the time required to achieve a high quality schedule will be excessively large and impractical, thus the quality might be sacrificed by ending the optimization process prematurely. It is, therefore, important to use a fast temperature simulation approach.

As previously discussed, the temperature simulation is based on a thermal model and a technique to solve the model response to the given input power profile. The input power consists of the static power and the dynamic power. The static power depends on the chip and on the temperature, while the dynamic power depends on the chip and on the input test sequence.

Both the static power and the dynamic power are time-variant, but for practicality reasons, it is commonly assumed that the power is constant 
during a simulation cycle ${ }^{3}$ (a discrete-time model is assumed). Therefore, in the following we focus on a single simulation cycle in which the input power is constant. The input power is updated with new static and dynamic power values, based on the results of the previous simulation cycle and then the simulation for the next cycle is performed.

The thermal model was previously discussed in section 2.6. Equation 2.6.1 is repeated below for convenience.

$$
\boldsymbol{A} \times \frac{d}{d t} \boldsymbol{\Theta}+\boldsymbol{B} \times \boldsymbol{\Theta}=\boldsymbol{P}
$$

Assume that the thermal model consists of $W$ nodes and $C$ is the number of cores $(C \leq W)$. The properties of the thermal model are encapsulated into two $W \times W$ matrices $\boldsymbol{A}$ and $\boldsymbol{B} . \boldsymbol{\Theta}$ and $\boldsymbol{P}$ are $W \times 1$ temperature and power vectors. The mathematical representation of this model (equation 4.6.1) is a system of linear constant-coefficient differential equations and therefore it is a linear time-invariant (LTI) system [Oppenheim97]. In fact the thermal model is a linear time-invariant lumped element model and both the heat capacities (captured in matrix $\boldsymbol{A}$ ) and thermal conductivities (captured in matrix $\boldsymbol{B}$ ) are linear and time invariant.

The other part of the temperature simulation is to solve the model in order to find its response to the input power. Usually, the simulation time is divided into smaller intervals in which the power could be assumed to be fixed. Then equation 4.6.1 is solved iteratively for each interval.

In order to solve equation 4.6.1 there are two distinct approaches, the numerical approximation and the closed form solution. The numerical approximations are usually done with very small intermediate steps, and as a result, the complete temperature curve for the interval is constructed.

HotSpot uses the Runge-Kutta method for numerical approximation [Huang06]. Though only the temperature at the end of the interval is registered, many points of the temperature curve are calculated. Since we do not need such a detailed temperature curve and we only need the temperature at the end of the intervals, the equation is solved analytically in order to give the temperature at the end of the intervals in a closed form.

\footnotetext{
${ }^{3}$ Simulation cycle is explained in section 2.6.
} 
In addition to the granularity of the temperature curve, another important factor, which affects the simulation speed, is how frequently equation 4.6.1 is required to be solved. The scheduling technique presented in this thesis, requires large number of simulations. Note that the system is LTI. Moreover, the only changes in the inputs (within the simulation intervals) is scaling of the previous inputs. Therefore, the differential equation needs to be solved only once at the very beginning [Oppenheim97].

The responses to the scaled versions of the previous inputs are obtained by scaling (a matrix multiplication) the previous outputs. Since the computational cost of the scaling is less than the computational cost of solving the equation from scratch, a method which utilizes the LTI properties (i.e., scaling and superposition [Oppenheim97]) is faster than the Runge-Kutta method when numerous simulations are required.

In situations that the thermal simulator is invoked quite frequently, the input power is just being scaled from cycle to cycle, and the thermal model is kept unchanged, the closed form solution is faster that the numerical techniques. Therefore, we continue with the simulation approach which is based on the closed form solution. By using Laplace transform [Oppenheim97] and assuming that $\theta^{0}$ is the initial temperature vector and $\theta^{t}$ is the temperature at the end of an interval, the closed form solution is

$$
\begin{aligned}
\theta^{t}=\exp \left(-\boldsymbol{A}^{-1} \times \boldsymbol{B} \times t\right) \times \theta^{0}+ \\
+\left(\boldsymbol{I}-\exp \left(-\boldsymbol{A}^{-1} \times \boldsymbol{B} \times t\right)\right) \times \boldsymbol{B}^{-1} \times \boldsymbol{P}
\end{aligned}
$$

Where $\boldsymbol{I}$ is the identity matrix of size $W$ and $t$ is the length of the interval. Now, $\boldsymbol{\alpha}$ and $\boldsymbol{\beta}$ matrices are defined as follows.

$$
\begin{aligned}
& \boldsymbol{\alpha}=\exp \left(-\boldsymbol{A}^{-1} \times \boldsymbol{B} \times t\right) \\
& \boldsymbol{\beta}=(\boldsymbol{I}-\boldsymbol{\alpha}) \times \boldsymbol{B}^{-1}
\end{aligned}
$$

With the help of $\boldsymbol{\alpha}$ and $\boldsymbol{\beta}$, equation 4.6.2 could be written as

$$
\theta^{t}=\boldsymbol{\alpha} \times \theta^{0}+\boldsymbol{\beta} \times \boldsymbol{P} .
$$

Equation 4.6 .5 could be understood intuitively by thinking about the system being LTI. According to the superposition principle, the effects of the initial value and the input power will add up, thus the plus sign between the two terms. The scaling property of the system could also be verified 


\section{Chapter 4}

rapidly, as the scaling of an input, $\theta^{0}$ or $\boldsymbol{P}$ with a certain factor, will scale its own effect by the same factor.

The temperature simulation is done in two phases, an initialization phase and then the operational phase. In the initialization phase the model is invoked and based on it $\boldsymbol{\alpha}$ and $\boldsymbol{\beta}$ are computed (this is shown in Figure 4.5.2 in regard to the overall scheduling method). The operational phase is the iterative computation of the temperatures for different times using equation 4.6.5. Since the thermal model is time invariant, the initialization is done only once at the very beginning of the design process. Throughout the offline scheduling phase, only the iterative computations are performed.

In the closed form solution, the most computationally expensive part is the matrix exponential for $\boldsymbol{\alpha}$ which is a part of equation 4.6.3. The matrix exponential could be computed using numerical methods such as Padé approximation [Higham05]. In fact the initialization phase for the closed form solution includes calculating equation 4.6 .3 and therefore it is very time consuming. However, the operational phase only includes computing equation 4.6 .5 and therefore it is fast.

On the other hand, for the Runge-Kutta approach [Press07], the initialization is fast since there is no need for computations which are as heavy as equation 4.6.3. However, the operational phase is slow since the equation is required to be solved in many fine steps through large number of intermediate time instances. The conclusion is that the Runge-Kutta method is faster for limited number of simulations and the closed form method is faster for large number of simulations. The experiments in section 4.7.1 will support this statement.

\subsection{Experimental Results}

Two distinct contributions in chapter 4 are the temperature simulation approach and the adaptive scheduling technique. These are experimentally evaluated in this section. All experiments are performed on a desktop computer with Intel ${ }^{\circledR}$ Xeon ${ }^{\circledR}$ W 3520 processor and 8 GB of memory. The experiments for temperature simulation are presented first.

\subsubsection{Fast Temperature Simulation Approach}

A temperature simulation approach based on the closed form solution is suggested in section 4.6 in order to increase the simulation speed. The 
problem with numerical approximation approaches for temperature simulation is that they are very slow for large number of simulation cycles especially when there are a large number of cores. Temperature simulations for a SoC with 100 cores and for different numbers of simulation cycles are performed using the proposed approach and using HotSpot [Huang07], and the CPU times are plotted in Figure 4.7.1a.

The numerical approximation approaches, such as the one used by HotSpot, perform faster than the suggested approach for a small number of simulation cycles. But for simulations longer than 1700 cycles, the proposed approach is faster than HotSpot, as shown in Figure 4.7.1a. In general, this difference increases with a rate close to 0.011 second per simulation cycle and it reached a CPU time difference of 100 seconds for 10000 simulation cycles. This is important since for every edge in every candidate schedule tree temperature simulation is performed for the number of test cycles plus cooling cycles.

Temperature simulations are performed using the proposed approach and using HotSpot [Huang07] for 10000 simulation cycles for different numbers of cores, and the CPU times are plotted in Figure 4.7.1b. In general, the CPU time difference increases rapidly with the number of cores and the difference reaches 100 seconds for 100 cores. This is also important, since achieving a good schedule in reasonable time becomes infeasible with a small increase in the number of cores, when the slower approach is in use.
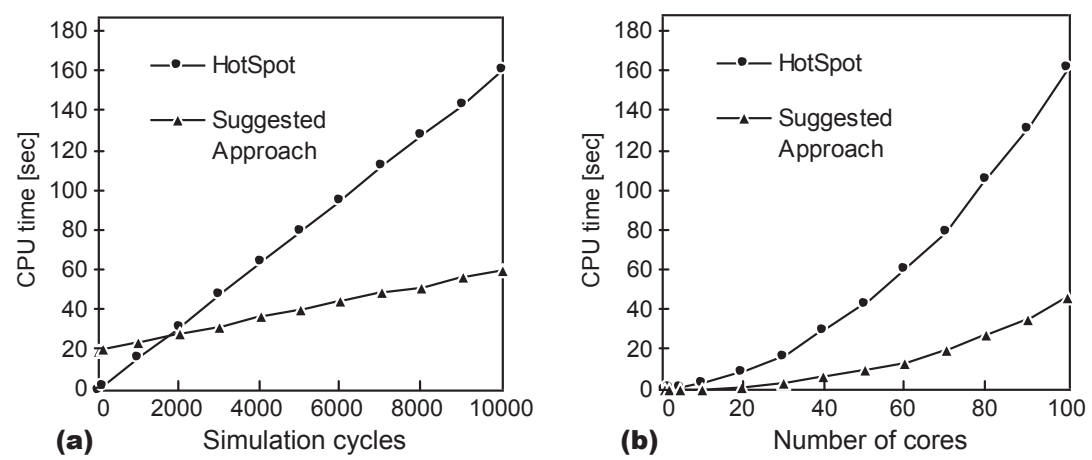

Figure 4.7.1 CPU times for temperature simulation

HotSpot and the suggested approach. The simulations are performed (a) for 100 cores for different numbers of simulation cycles and (b) for 10000 simulation cycles for different numbers of cores. 


\subsubsection{Adaptive Test Scheduling Technique}

The proposed adaptive SoC test scheduling technique is experimentally evaluated in this section. The first set of experiments is performed on SoCs with different number of cores and the CPU times are reported. Then, experiments are done for ITC'02 [Marinissen02] benchmark chips with random test switching activities generated using a Markov chain similar to [Yao11c]. Finally, an experiment is performed for the d695 benchmark chip from ITC' 02 with real switching activities based on real test data from [Samii06]. The costs of the test schedules and the test schedule sizes are reported for the last two sets of experiments. The experimental setup is briefly introduced at the beginning and then the results are presented.

The static power is computed using the temperature dependent model given in [Liao05]. The temperature simulations are performed using the approach proposed in section 4.6. The spatial temperature error is assumed to have normal distribution $\mathcal{N}(0,10)$ ranging from $-20^{\circ} \mathrm{C}$ to $+20^{\circ} \mathrm{C}$ with a resolution of $0.5^{\circ} \mathrm{C}(M=80)$. The temporal temperature error is also assumed to have a normal distribution $\mathcal{N}(0,10)$ ranging from $-40^{\circ} \mathrm{C}$ to $+40^{\circ} \mathrm{C}$ with a resolution of $0.5^{\circ} \mathrm{C}$. It is assumed that there are twenty temperature error clusters $(L=20)$.

The balancing coefficient is assumed to be equal to ten $(B C=10)$. It is assumed that each entry in a linear schedule table occupies 64 bits and each entry in a branching table per core per edge occupies 32 bits. For example a node with two succeeding edges for a SoC with two cores, occupies $(2 \times 2 \times 32)=128$ bits.

The first set of experiments is performed on a number of SoCs with different number of cores ranging from five to 50 cores. Markov chains are used to generate random test switching activity sequences having random averages and random lengths. The experiments are performed for at least five randomly generated sets of tests for each chip and the average CPU times are reported in Table 4.7.1. Note that even for a 50-core SoC, the CPU time remains in an affordable range.

Table 4.7.1 CPU times for SoCs with different number of cores

\begin{tabular}{crrrrrrrrrr}
\hline Number of Cores & 5 & 10 & 15 & 20 & 25 & 30 & 35 & 40 & 45 & 50 \\
CPU time [Sec] & 9 & 46 & 52 & 132 & 208 & 308 & 590 & 762 & 1141 & 1367 \\
\hline
\end{tabular}


The second set of experiments is performed on ITC'02 SoCs with randomly generated test switching activities similar to the first set of experiments but this time tests for a chip have constant power averages and length. The proposed technique is compared with the two methods proposed in [Aghaee10]. The first one is an Offline method which uses only one linear schedule and the other is a Hybrid method which selects a linear schedule (out of a set of pre-generated schedules) only once during the test process. The test costs offered by the Offline and Hybrid methods proposed in [Aghaee10] and by the proposed technique in this chapter are computed using the metric given in equation 4.3.4 and are reported in Table 4.7.2.

Column 1 is the name of the ITC' 02 circuits. Columns 2 and 3 are the costs (based on equation 4.3.4) for schedules generated by Offline and Hybrid approaches proposed in [Aghaee10], respectively. The costs of the schedules generated by the proposed adaptive approach are reported in column 4 in Table 4.7.2. The percentage reduction in cost achieved by the Hybrid and adaptive approaches compared with the Offline approach are reported in columns 5 and 6 , respectively. Column 7 is the percentage reduction in cost achieved by the proposed adaptive approach compared with the Hybrid approach. The adaptive method proposed here reduces the cost by $76 \%$ over the Offline method and $43 \%$ over the Hybrid method. This demonstrates the advantage of the proposed adaptive method.

The ATE memory occupied to store the schedules (i.e., the schedule size) is reported in Table 4.7.3. The cost reduction comes with increase in the

Table 4.7.2 Test cost for test scheduling techniques

\begin{tabular}{|c|c|c|c|c|c|c|}
\hline \multirow{2}{*}{$\begin{array}{l}\text { ITC'02 } \\
\text { chips }\end{array}$} & \multicolumn{3}{|l|}{ Costs } & \multicolumn{2}{|c|}{$\begin{array}{l}\text { Percentage } \\
\text { reduction relative to } \\
\text { the Offline }\end{array}$} & \multirow{2}{*}{$\begin{array}{l}\text { Percentage } \\
\text { reduction relative } \\
\text { to the Hybrid } \\
\text { Proposed } \\
\end{array}$} \\
\hline & Offline & Hybrid & Proposed & Hybrid & Proposed & \\
\hline$a 586710$ & 1.44 & 0.56 & 0.54 & 61 & 62 & $\overline{4}$ \\
\hline$d 281$ & 0.69 & 0.45 & 0.03 & 35 & 96 & 93 \\
\hline$d 695$ & 0.50 & 0.12 & 0.06 & 76 & 88 & 50 \\
\hline$f 2126$ & 2.71 & 1.39 & 0.51 & 49 & 81 & 63 \\
\hline$g 1023$ & 5.09 & 4.27 & 1.99 & 16 & 61 & 53 \\
\hline h953 & 0.46 & 0.14 & 0.11 & 70 & 76 & 21 \\
\hline p22810 & 1.22 & 0.70 & 0.69 & 43 & 43 & 1 \\
\hline p34392 & 0.75 & 0.72 & 0.06 & 4 & 92 & 92 \\
\hline p93791 & 1.02 & 0.13 & 0.08 & 87 & 92 & 38 \\
\hline$q 12710$ & 1.32 & 0.40 & 0.23 & 70 & 83 & 42 \\
\hline$t 512505$ & 0.48 & 0.23 & 0.13 & 52 & 73 & 43 \\
\hline$u 226$ & 1.05 & 0.43 & 0.37 & 59 & 65 & 14 \\
\hline Average & & & & 52 & 76 & 43 \\
\hline
\end{tabular}


schedule size because of increased number of linear schedules and branching tables, which consume ATE memory space. The average increase in schedule size compared to Offline is $87 \%$ for Hybrid and 308\% for the proposed adaptive method. When compared to Hybrid, the average schedule size increase for the proposed method is $117 \%$. The increase in the usage of ATE memory (as given in Table 4.7.3) refers only to the memory space used to store the schedule. This is usually small, compared with the memory space used to store the test patterns. Therefore a large increase in the schedule size is very likely to be translated into a small increase in the usage of the ATE memory as a whole.

The proposed scheduling method will utilize the available ATE memory even if a very small reduction in cost (e.g., from 0.70 to 0.69 for p22810 in Table 4.7.2) is achieved. Since the number of nodes contributes to the scaled cost function (equation 4.5.1), a larger schedule will not be generated (e.g., 195\% larger for p22810 in Table 4.7 .3 compared with hybrid solution) if it does not reduce the cost compared with a smaller schedule.

The ATE memory constraint will affect the quality of the adaptive test schedules. The proposed algorithm will not generate even an offline schedule when the available memory is too small to accommodate it. By increasing the available ATE memory, first an offline schedule and then a hybrid schedule will be generated. With the further increase of the available memory, better schedules with lower costs will be generated. This trend continues until the cost reaches a minimum beyond which

Table 4.7.3 ATE memory utilized only for schedule

\begin{tabular}{|c|c|c|c|c|c|c|}
\hline \multirow{2}{*}{$\begin{array}{l}\text { ITC'02 } \\
\text { chips }\end{array}$} & \multicolumn{3}{|c|}{$\begin{array}{l}\text { Utilized memory for } \\
\text { schedule [bit] }\end{array}$} & \multicolumn{2}{|c|}{$\begin{array}{l}\text { Percentage increase } \\
\text { relative to the Offline }\end{array}$} & \multirow{2}{*}{$\begin{array}{l}\text { Percentage increase } \\
\text { relative to the Hybrid } \\
\text { Proposed } \\
\end{array}$} \\
\hline & Offline & Hybrid & Proposed & Hybrid & Proposed & \\
\hline$a 586710$ & 1216 & 1888 & 4768 & 55 & 292 & 152 \\
\hline$d 281$ & 1088 & 1280 & 2624 & 18 & 141 & 105 \\
\hline$d 695$ & 1280 & 2176 & 3392 & 70 & 165 & 54 \\
\hline$f 2126$ & 704 & 960 & 2368 & 36 & 236 & 147 \\
\hline g1023 & 576 & 1088 & 4480 & 89 & 678 & 312 \\
\hline h953 & 576 & 1088 & 1472 & 89 & 155 & 35 \\
\hline p22810 & 704 & 1888 & 5568 & 168 & 691 & 195 \\
\hline p34392 & 832 & 1472 & 2688 & 77 & 223 & 83 \\
\hline p93791 & 704 & 1920 & 3136 & 173 & 345 & 63 \\
\hline$q 12710$ & 640 & 1024 & 1664 & 60 & 160 & 62 \\
\hline t512505 & 1152 & 2336 & 3712 & 103 & 222 & 59 \\
\hline$u 226$ & 320 & 672 & 1568 & 110 & 390 & 133 \\
\hline Average & & & & 87 & 308 & 117 \\
\hline
\end{tabular}


further cost reduction is impossible. The minimum cost is usually dictated by the branching overheads (time to read sensors and react accordingly).

The reduction of the cost with the increase of the memory limit is shown in Table 4.7.4. The memory limit is increased in eight steps. It is expected that the increase in the memory limit improves the cost before it reaches the saturation limit. The saturation limit for this set of experiments is equal to 1320 . Memory sizes and limits for the schedule are given in bytes. The CPU time increases in general with the increase of memory limit. This trend continues even if the cost is not improved (after the saturation) since the algorithm has more space to search and thus it takes more time. The costs and sizes are normalized to the first working schedule (row 4) and reported in columns 4 and 5 of Table 4.7.4.

The last experiment is performed on d695 (one of the ITC'02 chips) using the real test switching activities. The costs and schedule sizes are reported in Table 4.7.5. The Hybrid method improves the cost compared to Offline method by $59 \%$ while the proposed adaptive technique achieves a reduction of $71 \%$. The proposed technique improves the cost by $30 \%$ over the Hybrid method. The schedule size for the proposed method is $169 \%$ and $49 \%$ larger than Offline and Hybrid, respectively. As we expected, the improvement in cost and the increase in the schedule size are in the ranges suggested before by the second set of experiments.

As previously mentioned, the effect of increased schedule size on the total consumed ATE memory is small. For example consider the experiments with the d695 chip with real switching activities. The size of the schedule

Table 4.7.4 Costs and utilized memory volumes for different memory limits

\begin{tabular}{|c|c|c|c|c|c|}
\hline \multirow{2}{*}{$\begin{array}{l}\text { Memory } \\
\text { limit }\end{array}$} & \multicolumn{5}{|l|}{ Results } \\
\hline & Cost & Size & Cost (\%) & Size (\%) & CPU time (H:M:S) \\
\hline 300 & \multicolumn{4}{|c|}{ Aborted, memory limit is too tight } & $1: 03: 42$ \\
\hline 500 & 3.3875 & 460 & 100.00 & 100.00 & $3: 15: 21$ \\
\hline 750 & 3.3875 & 460 & 100.00 & 100.00 & $3: 34: 20$ \\
\hline 1000 & 2.9389 & 920 & 86.76 & 200.00 & $3: 41: 03$ \\
\hline 1250 & 2.9389 & 920 & 86.76 & 200.00 & $3: 48: 47$ \\
\hline 1500 & 2.7170 & 1320 & 80.21 & 286.96 & $3: 53: 52$ \\
\hline 1750 & 2.7170 & 1320 & 80.21 & 286.96 & $3: 59: 12$ \\
\hline 2000 & 2.7170 & 1320 & 80.21 & 286.96 & $4: 04: 16$ \\
\hline
\end{tabular}


Table 4.7.5 Cost and ATE memory utilized for schedule for d695

\begin{tabular}{|c|c|c|c|c|c|c|}
\hline & \multirow[b]{2}{*}{ Offline } & \multirow[b]{2}{*}{ Hybrid } & \multirow[b]{2}{*}{ Proposed } & \multicolumn{2}{|c|}{$\begin{array}{l}\text { Percentage change } \\
\text { relative to the Offline }\end{array}$} & \multirow{2}{*}{$\begin{array}{l}\text { Percentage } \\
\text { change relative } \\
\text { to the Hybrid } \\
\text { Proposed }\end{array}$} \\
\hline & & & & Hybrid & Proposed & \\
\hline Cost & 20.84 & 8.53 & 5.93 & -59 & -71 & -30 \\
\hline $\begin{array}{l}\text { Utilized } \\
\text { memory for } \\
\text { schedule [bit] }\end{array}$ & 2688 & 4992 & 7232 & +86 & +169 & +49 \\
\hline
\end{tabular}

for the adaptive solution is approximately $7 \mathrm{Kbit}$ while the test size is approximately $1324 \mathrm{Kbit}$. Therefore the percentage increase in total utilized ATE memory from the offline solution to the adaptive solution is $0.34 \%$. This means that the adaptive method achieves $71 \%$ reduction in cost relative to the offline method, with a small expense of $0.34 \%$ increase in the occupied ATE memory.

\subsection{Adaptive Multi-Temperature Testing}

As previously discussed in section 3.5, temperature-dependent defects are a challenge for achieving high test quality for advanced SoC. The existing multi-temperature test scheduling methods optimize the test schedule for the shortest test application time while making sure that the tests are applied inside the specified temperature ranges [He10, Yao11b]. These methods neglect the temperature deviations that are mainly caused by process variation. Therefore, a large process variation implies a decreased number of chips that are tested within the specified temperature ranges, which will reduce the effectiveness of the tests and, in the worst case, may lead to damage of the chips due to overheating.

In order to maximize the chances that the tests are applied within the intended temperature ranges, static schedules should be designed pessimistically. In this case, a large process variation implies a very long test application time due to the intensive use of the heating and cooling intervals. This means that the chips under test are heating up/cooling down more than actually needed in order to make sure that it is warm/cold enough for the majority of the chips. This is similar to the discussions about the safety margins in section 3.3. A detailed discussion and analysis of safety margins can be found in [Aghaee10].

The test application time for multi-temperature testing is much longer than the normal testing and therefore the test cost is higher [He10, Yao11b]. 
This becomes a serious cost issue, in particular in situations that the normal test application time is already very long, as it is for advanced SoCs. The proposed methods in [He10, Yao11b] provide satisfactory results when the temperature at a certain test cycle could be assumed to be identical for all chips of the same design.

However, advanced SoCs manufactured with deep submicron technologies are likely to have different temperatures at the same test cycle because of process variation. The negative effect of temperature variations on the thermal safety of the SoCs during test is addressed by the scheduling methods proposed in the previous sections. These methods try to limit the cores' maximum temperatures so that the test damages caused by overheating during the test process are minimized. Similar techniques can be applied in the context of multi-temperature testing. We have proposed a technique to generate test schedules so that the tests have a large likelihood of being applied at the correct temperatures [Aghaee14b]. Here, we briefly explain the methodology.

As mentioned before, the adaptive multi-temperature testing is similar to the thermal-safe approach introduced in this chapter. The key difference is that heating stimuli and cooling intervals are used to bring the temperature inside the required range. Only then the tests can be applied. Due to testing, the temperature may exceed the high limit. In this case, the testing is paused at an appropriate moment and then cooling intervals are introduced. On the other hand, if the temperature falls below the low limit, testing is paused and a heating sequence is introduced, instead.

The thermal-aware techniques only support one temperature limit which is the overheating limit and exceeding it adds to the overall cost of testing by increasing the number of overheated chips. On the other hand, multitemperature techniques have to consider the upper limit and the lower limit of the temperature interval characteristic to each test. Exceeding these limits results in test escapes which means that some defective chips may not be detected. This new contributor to the testing costs is defined in [Aghaee14b] and is added to the costs already defined in section 4.3.

Having to handle a low temperature limit adds to the complexity of the techniques presented in section 4.5. Representative temperature error is introduced in section 4.5.2. Every chip cluster is represented by a dedicated representative temperature error. In fact this representative value is defined and optimized with regard to the high temperature limit. Therefore, having 
an additional low limit means that another representative is required with regard to this low limit.

The proposed adaptive multi-temperature technique is explained in details in [Aghaee14b] and is supported by experiments. The overall cost that captures costs related to the test application time, overheating, and out of required-range testing is minimized. Required ATE memory, CPU times, and cost dependency on the amount of process variation are also reported in [Aghaee14b].

\subsection{Remarks}

Although the proposed adaptive techniques are developed to handle PVrelated temperature errors, they can be adopted to handle other non-ideal situations. Such situations may happen during in-field testing, where the initial and ambient temperatures (among other parameters like voltage) may vary. Acquired temperature data using on-chip (or on-board) sensors help to select the most appropriate linear-schedule and minimize the costs.

The temperature error that is explained in section 4.4 is discussed in more details here. In order to distinguish between the effects of the process variation and other undesirable thermal effects, four different temperatures can be defined. The first one is expected temperature that is the temperature of a normal chip which is not affected by undesirable thermal effects (including process variation). The expected temperature is an abstract concept and its exact value could not be acquired. The second one is simulated temperature that is the temperature computed by simulation. The aim of simulation is to compute the expected temperature and therefore, ideally, the simulated temperature is equal to the expected temperature. The third one is actual temperature that is the actual realworld temperature. Its exact value is usually impossible to acquire due to measurement errors. The fourth and last one is measured temperature that is the measured temperature using temperature sensors.

Based on the above definitions, three different temperature errors can be identified. The first one is simulator error that is the difference between the expected temperature and the simulated temperature. The inaccuracies in the thermal model and algorithms which the simulator is based on, contribute to this error. The second one is measurement error that is the difference between the actual temperature and the measured temperature. The inaccuracies in the sensor technologies contribute to it. The third and 
last one is variation error that is defined as the difference between the actual temperature and the expected temperature. This error has various sources including process variation, ambient temperature fluctuations, and voltage variations.

Even though the temperature simulator errors and sensor measurement errors are not addressed explicitly in this thesis, in practice when the temperature error model is being tuned empirically, a considerable amount of these errors will also be covered. There still might be small residual errors which are not captured by the temperature error model. These small residual errors are addressed by introducing a small safety margin (e.g., a slightly lower overheating limit than the actual overheating limit is used in practice). The effect of this small safety margin on cost is negligible as discussed in [Aghaee10].

The focus of this chapter is process variation which mainly contributes to the variation error and therefore in this thesis we focus on this category of errors. To avoid these complications, we usually consider the temperature error as the difference between the expected temperature which is estimated by simulation and the actual temperature which is measured by sensors. Meaning that we assume that the actual temperature and measured temperature are equivalent. Moreover, we assume that the simulated and expected temperatures are equivalent. Nevertheless, our proposed approaches can be used to address other errors like the simulator errors and the measurement errors, although they are not explicitly designed for addressing these types of errors.

\subsection{Conclusions}

This chapter mainly presents an adaptive SoC test scheduling technique to deal with spatial and temporal temperature deviations, caused by process variations in deep submicron technologies. Mitigating the negative variation effects on the multi-temperature testing, reported in [Aghaee14b], is similar to the thermal-safe testing and therefore is just briefly discussed above.

The key contribution of this chapter is an algorithm to generate a set of efficient test schedules, each corresponding to a different thermal behavior of different cores during test. The on-chip temperature sensors are used to monitor the actual temperatures of the different cores and to guide the 


\section{Chapter 4}

selection of the corresponding test schedules accordingly, during the test. This way, the overall test efficiency will be improved considerably.

The proposed technique consists of two distinct algorithms, the test scheduler and the thermal simulator. The temperature-aware test scheduler is a constructive algorithm which generates tree-based test schedules by putting the optimized sub-trees together. Sub-tree optimization is basically a chip-clustering algorithm which involves a linear test scheduling algorithm. A new sub-tree scheduling algorithm is proposed here. The linear scheduling algorithm requires a thermal simulator in its main loop. A fast temperature simulation approach is proposed in order to speed up the temperature-aware test scheduling algorithm.

The proposed adaptive test scheduling technique generates processvariation and temperature aware test schedules for SoCs with a large number of cores. The algorithm has a relatively short run-time and generates high quality test schedules. The proposed technique has been experimentally evaluated using a number of experiments including ITC'02 benchmark SoCs. 


\subsection{Notations and Abbreviations}

\begin{tabular}{|c|c|}
\hline Notation & Description \\
\hline$A$ & Capacitances vector in the thermal model \\
\hline ATS & Applied Test Size \\
\hline$B$ & Resistances vector in the thermal model \\
\hline$B C$ & Balancing Coefficient \\
\hline Branching table & $\begin{array}{l}\text { The table that determines with which linear schedule table a } \\
\text { specific chip should be tested. (See the example in section } 4.2 \text { ) }\end{array}$ \\
\hline$C$ & Number of cores \\
\hline Chip cluster & $\begin{array}{l}\text { A group of chips with similar thermal behavior that are tested } \\
\text { with the same Linear schedule table. A chip cluster corresponds } \\
\text { to an edge in the schedule tree. }\end{array}$ \\
\hline Chip-cluster border & $\begin{array}{l}\text { The border line between two Chip clusters. For two adjacent } \\
\text { Chip clusters the border is a set of natural numbers, each } \\
\text { corresponding to an individual core. A border represents a } \\
\text { particular error value. (See section 4.5.4) }\end{array}$ \\
\hline Chip clustering & $\begin{array}{l}\text { Finding the optimal partitioning of the } C \text {-dimensional error } \\
\text { space into an already known number of Chip clusters for the } \\
\text { nodes of a tree. (See full explanation in section } 4.5 .4 \text { ) }\end{array}$ \\
\hline$C T F$ & Cost of the Test Facility per time unit \\
\hline EATS & Expected Applied Test Size \\
\hline$E B$ & (temperature) Error-clusters Borders \\
\hline ECCP & Error Cell Change Probabilities \\
\hline$E C C P^{\text {before }}$ normalization & ECCP before being normalized \\
\hline$E C L$ & Error-Cell Labeling \\
\hline$E C P$ & Error-Cells Probabilities \\
\hline$E C P^{\text {after branching }}$ & ECP just after branching \\
\hline$E C P^{\text {after overheating }}$ & $E C P$ just after overheating \\
\hline
\end{tabular}




\section{Chapter 4}

\begin{tabular}{|c|c|}
\hline Notation & Description \\
\hline$E C P^{\text {after temporal changes }}$ & $\begin{array}{l}\text { ECP after temporal changes (according to temperature error } \\
\text { model) }\end{array}$ \\
\hline Error cluster & $\begin{array}{l}\text { A range of error values which are to be treated as one single error } \\
\text { value. Error clusters are separated by Error-clusters Borders, } E B \text {. }\end{array}$ \\
\hline ETAT & Expected Test Application Time \\
\hline ETOP & Expected Test Overheating Probability \\
\hline ETTpS & Effective Test Time per Second \\
\hline$H T T$ & High Temperature Threshold \\
\hline$I$ & Identity matrix \\
\hline Knee & $\begin{array}{l}\text { The point that the output is equal to the input and not equal to one, } \\
\text { in the proposed reciprocal limiter. }\end{array}$ \\
\hline$L$ & Number of temperature error clusters \\
\hline Linear schedule table & $\begin{array}{l}\text { A schedule that specifies stop/start times for the test of each and } \\
\text { every core, individually. This will correspond to an edge or to a } \\
\text { single Chip cluster. (See the example in section 4.2) }\end{array}$ \\
\hline$L O P$ & Leaves' Overheating Probabilities \\
\hline$M$ & Number of temperature error values \\
\hline$N$ & Number of nodes in a tree \\
\hline NATS & Nodes' Applied Test Sizes \\
\hline$N C F$ & Normalized Cost Function \\
\hline$N C L$ & Node's Cluster Label \\
\hline $\mathrm{NnOP}$ & Node's not Overheating Probability \\
\hline Node & $\begin{array}{l}\text { A node in the schedule tree that corresponds to the ending of a } \\
\text { Linear schedule table (i.e., a place that branching is possible). }\end{array}$ \\
\hline$N P$ & Nodes' Probabilities \\
\hline$N P T$ & $\begin{array}{l}\text { expected Number of Partial Trees, similar to the current partial } \\
\text { tree, that are required to construct the complete schedule tree }\end{array}$ \\
\hline
\end{tabular}




\begin{tabular}{|c|c|}
\hline Notation & Description \\
\hline NTAT & Nodes' Test Application Times \\
\hline$N T T$ & Normalized Test Throughput \\
\hline$P$ & Power vector \\
\hline Partial cost function & NCF evaluated for a part of the schedule tree (e.g., a sub-tree). \\
\hline POC & Price of One Chip \\
\hline PSO & Particle Swarm Optimization \\
\hline PTOP & Predicted Test Overheating Probability \\
\hline$Q$ & Number of leaf nodes \\
\hline$S$ & Number of succeeding edges for a node \\
\hline$S C F$ & $\begin{array}{l}\text { Scaled Cost Function that is used to select the unfinished trees } \\
\text { out of a group of offspring trees. }\end{array}$ \\
\hline$S T$ & Simulated Temperature \\
\hline STEP & Spatial Temperature Error Probabilities \\
\hline$T A M$ & Test Access Mechanism \\
\hline$T A T$ & Test Application Time \\
\hline $\begin{array}{l}\text { Temporal error } \\
\text { period }\end{array}$ & $\begin{array}{l}\text { The period for the discrete-time temperature error model. The } \\
\text { error values are updated regularly with a frequency equal } \\
\text { to } 1 / \text { Temporal_error_period. (See section } 4.4 \text { ) }\end{array}$ \\
\hline$T E V$ & Temperature Error Values \\
\hline THT & Test Handling Time \\
\hline TOP & Test Overheating Probability \\
\hline$T T$ & Test Throughput \\
\hline TTEP & Temporal Temperature Error Probability \\
\hline$W$ & Number of nodes in the thermal model \\
\hline
\end{tabular}




\section{Chapter 4}

\begin{tabular}{ll}
\hline Notation & Description \\
\hline $\boldsymbol{\alpha}$ & Transfer matrix for initial temperatures \\
\hline $\boldsymbol{\beta}$ & Transfer matrix for power values \\
\hline$\theta^{0}$ & Temperatures vector in thermal model \\
\hline$\theta^{t}$ & Initial temperatures \\
\hline$\theta_{w}$ & Temperatures at the end of the interval of size $t$ \\
\hline$\lambda$ & Temperatures at $t$-th time sample (in section 4.6) \\
\hline
\end{tabular}




\section{Chapter 5 Temperature-Gradient Based Burn-In and Test Scheduling}

Large temperature gradients (e.g., temperature difference between two adjacent cores) exacerbate various types of defects including early-life failures and delay faults. The capability to detect these temperaturegradient induced defects is crucial for advanced SoCs. In particular, 3DSICs exhibit considerably larger temperature gradients compared with normal ICs (for example, three times is reported in [Plas10]) and therefore temperature-gradient based test is crucial for them.

The gradients are captured and represented by temperature maps. This chapter presents schedule based techniques to enforce temperature maps on the IC. A temperature map specifies the temperatures for different sites (e.g., cores) in the IC at a given time-point. It usually specifies the high and the low temperature limits for each site. Alternatively, the intermediate temperatures (half-way from low limit to high limit) can be used to represent a temperature map, in particular if the difference between high and low limits are similar for all sites.

\subsection{Introduction}

\subsubsection{Test for Early-Life Failures}

Burn-in is a common way of accelerating and detecting early-life failures and it should be done with low cost in a reasonably short time. For this purpose, usually, the dies are operated at elevated temperature and voltage. The elevated temperature and voltage speed up the aging and wear mechanisms so that the dies experience their early life before testing. The wear mechanisms that are speeded up include metal stress voiding and electromigration, metal slivers bridging shorts, as well as gate-oxide wearout and breakdown [Semenov03]. 
Recently, several studies have, however, shown that some wear mechanisms are speeded up more efficiently by large temperature gradients rather than the high temperature itself. A temperature-gradient induced wear mechanism is identified in [Smorodin08] which shows that a metal layer elevation develops rapidly on the sites that experience large temperature gradients. Moreover, in the atomic flux equation that models the electromigration, temperature gradient is present directly and also indirectly through its effect on the mechanical-stress gradient [Pak11].

Therefore, a burn-in process that has not created the appropriate thermal scenarios will not sufficiently speed up the formation of the defects and, consequently, such early-life defects will go undetected. In order to prevent these test escapes, it is necessary to introduce a burn-in process that enforces appropriate temperature scenarios on the IC. This necessity is more urgent for the ICs that suffer from large temperature gradients, such as $3 \mathrm{D}-\mathrm{SIC}$.

3D-SIC technology, similar to other deep submicron technologies, suffers from high power densities. Additionally, power densities are considerably higher in the test mode compared to the functional mode, in particular for core-based designs. Consequently overheating may damage the ICs under test. This means that the application of test stimuli to ICs can raise their temperatures beyond their tolerable limits. This often undesirable effect is, however, utilized in this thesis to heat up the IC for burn-in.

In our case the stimuli are not necessarily actual test patterns. Instead, they could be specially generated sequences which cause large switching activities. Such stimuli are called heating sequences. The use of the heating sequences to heat up the IC from inside means that special equipment for heating the IC from outside are not necessary. This will lead to large reduction of cost, and also allow for the generation of necessary temperature gradients.

Some temperature gradients might be enforced on an IC by applying appropriate inputs to the IC's input ports in the functional mode. This might work, to some extent, for 2D ICs, since from the functional point of view all the required circuitry, including the input ports, are fabricated and available when the IC enters the test process. For 2D ICs, there are usually two possible stages for burn-in: Wafer-Level Burn-In (WLBI) which is performed before packaging and Die-Level Burn-In (DLBI) performed after packaging [Semenov03]. For 3D-SIC, however, there are more 
stages, including pre-bond, mid-bond, post-bond, and final stages [Taouil12].

Existence of the test stages before the IC is fully assembled is a key difference between the 2D and 3D-SIC burn-in process. In the case of 3DSIC, using input ports in the functional mode may benefit burn-in for the post-bond and the final stages similar to 2D ICs. But for the pre-bond or mid-bond stages, the inputs to the die or partially stacked dies are not necessarily the inputs to the IC. The input ports to the unit under test for 3D-SICs, before the final bonding, are likely to include a number of TSVs.

The TSVs and test equipment are not designed to support simultaneous application of functional signals, particularly to large number of TSVs (even though they might be designed to allow simple electrical tests for the TSV itself). Therefore, the use of the IC's ports for enforcing the temperature gradients is not possible for the pre-bond and mid-bond stages. Albeit this lack of access in the functional mode, TAM provides access to the cores, in the test mode [Ieee14a]. Therefore, the heating sequences could be applies using the TAM in order to enforce the desired gradients.

The necessity to utilize the TAM has yet another reason that is not specific to 3D-SICs. The thermal gradients in some maps might be placed in locations that cannot be properly stimulated through functional input ports. Such thermal maps can often be enforced if the TAM is used. The reason is that the TAM, in the test mode, provides direct access to cores; while in the normal operational mode, a core might be limited to receive inputs only from an adjacent core. Therefore, heating could be targeted toward a specific core using the TAM.

\subsubsection{Test for Delay Faults}

Advanced SoCs manufactured by 3D-SIC technology suffer from a considerably larger number of delay faults as compared with previous technologies [Deutsch12]. The causes for these delay faults include resistive bridges and vias, power droops, and cross-talk noise effects. Therefore, delay-fault testing is necessary to provide sufficient fault coverage [Patil07, Raina07]. A large number of pre-bond TSV defects are resistive in nature and, moreover, the mechanical stress caused by TSVs contributes also to delay faults [Chakrabarty12, Deutsch12]. Therefore, the expected number of delay faults for 3D-SIC is much larger than that of $2 \mathrm{D}$ ICs. 
Since temperature has a significant effect on delay, its impact should be taken into account for delay-fault test. A very important effect of temperature on signal integrity is its effect on the clock network [Bota04]. Delay faults usually occur because of increased clock skew and a major contributor to skew in 3D-SICs is temperature gradient [Monda107]. Since propagation delays depend on temperature, different temperatures on different sites (i.e., temperature gradients) result in different clock skews. Temperature gradients may reach up to $50^{\circ} \mathrm{C}$ in adjacent cores for normal operation and even higher during test [Borkar03, Bota04, Monda107]. Such large temperature gradients may lead to considerable clock skew and thus many delay faults.

Moreover, the difference between the temperature maps during the normal functional operation and temperature maps during test will result in nonrealistic delay faults [Bota04]. These delay faults usually happen because of increased clock skew. Therefore, in order to detect the realistic delay faults during the test, the test should be performed when the die has a temperature map which corresponds to a normal functional situation.

In order to test a die under the thermal conditions that correspond to reality, a simple technique is to operate the chip with realistic inputs so that the temperature map is created disregarding the test. Then start the test and go on with it as long as the thermal map maintains an acceptable difference with the specified thermal map. When the difference grows larger than accepted, the test is halted and the specified thermal map is re-created disregarding the test. Apart from being slow, this scenario has another problem in case of 3D-SIC.

As discussed in the previous section, usually a die in a 3D stack has a large number of TSVs as its input ports. The TSVs and test equipment are not expected to be designed to support simultaneous application of realistic signals, particularly to large number of TSVs. Therefore, it is not possible to use the IC's real functional inputs to create the specified thermal map for pre-bond and mid-bond tests. However, the test access mechanism can be utilized for this purpose. This will be further discussed in section 5.2.1. 
Besides creating realistic gradient scenarios to avoid test overkills, certain unreal $^{1}$ scenarios may help to detect certain early-life defects before causing further costs. As previously discussed, in the normal operational mode such unreal scenarios may not be achievable since not all involved cores are accessible. However, in the test mode, TAM may provide access to these involved cores.

As mentioned before, the temperature gradients in 3D-SICs are much larger than in 2D ICs [Plas10]. This will exacerbate temperature-gradient related issues including delay faults, in particular, for 3D-SIC. Therefore, the associated tests should be performed when the proper temperature maps are enforced. A temperature map specifies the appropriate temperatures for different sites (e.g., cores) in the IC. These temperatures are to be realized simultaneously in order to enforce the proper temperature gradients. The temperature maps are given along with their corresponding tests. Beside the gradient-based burn-in the other objective of this chapter is to introduce a technique to apply the tests while the corresponding maps are enforced on the IC.

\subsection{Temperature-Gradient Based Burn-In}

\subsubsection{Motivation and Problem Description}

As discussed earlier, a temperature map specifies the desired temperature values for different sites (e.g., cores). The temperature maps are to be given by the user, who studies the typical temperature-gradient induced failure mechanisms in an IC analytically or experimentally [Pak11, Smorodin08]. Each map corresponds to a particular temperature condition of an IC, such as large temperature differences between adjacent cores (i.e., large temperature gradients), that can accelerate aging for early-life failures or enlarge the delay fault effect so that they can easily be tested for. There might also be some locations in the ICs such that their temperatures are not important regarding the targeted defects. Such locations are indicated as don't-cares. Even though they are marked as don't-cares, their temperature should, however, be kept below the overheating limit (denoted by $\theta^{\text {overheating }}$ ) in order to prevent damage.

${ }^{1}$ Unreal gradients are scenarios that are not expected to happen during field operations. The opposite is "realistic gradients" that happen during normal operations. 
When the expected locations in the IC simultaneously have the temperature values that are specified by a map, it is said that that temperature map is enforced. The specified temperature maps should be enforced quickly. In case of burn-in, the temperatures should then be maintained for a given period of time to achieve the intended effect and for test it should be maintained as long as the corresponding tests are being performed.

Usually, there are many temperature maps that one would like to achieve and maintain. Therefore, it is important to achieve them rapidly whether the ICs start from the ambient temperature or from another map. The order of the maps has a considerable impact on the overall burn-in/test time and will be discussed in-depth later on in this chapter. For the time being, we assume that the maps order is given and focus on other aspects of the problem. In our work, a temperature map will be achieved by using heating sequences sent through the TAM. Moreover, it is assumed that no test is applied when an IC is kept under a temperature map for burn-in. This assumption will be relaxed in section 5.3 so that the tests can be applied when an IC is kept under a temperature map.

Assume that there are $M$ modules in an IC (on one or multiple dies) and their tests can be started and stopped independently (e.g., the modules are cores with core wrappers in a core-based design). In order to enforce the specified temperature maps, heating sequences are used to heat up some of the modules. The average power of the heating sequence is given by a real number, denoted by ${ }^{2} p_{m}^{H S}$ for module $m(0 \leq m<M)$. It is assumed that the TAM only affords $W$ (a positive integer number) modules to be tested simultaneously.

Assume that the desired temperature map is specified by a low temperature limit and a high temperature limit for each module and the don't-care modules are declared separately. For example, a temperature map specifies that module $m$ has a low temperature limit equal to $\theta_{m}^{L}$ and a high temperature limit equal to $\theta_{m}^{H}$.

The inputs to the proposed method include temperature maps, the IC's temperature model, the IC's electrical model (e.g., specification of the TAM and power-related specifications), switching activities of the heating sequences, ambient temperature $\left(\theta^{\text {ambient }}\right)$, and overheating limit

${ }^{2}$ A list of notations and abbreviations is provided in section 5.6. 
( $\left.\theta^{\text {overheating }}\right)$. The output is a schedule that guides the application of the heating sequences to the modules so that their temperatures move into the specified ranges and stay there.

As an example, consider an IC with 3 modules, $m_{0}, m_{1}$, and $m_{2}$. Assume that a temperature map is specified as $\theta_{0}^{H}=125^{\circ} \mathrm{C}, \theta_{0}^{L}=115^{\circ} \mathrm{C}, \theta_{1}^{H}=$ $95^{\circ} \mathrm{C}, \theta_{1}^{L}=85^{\circ} \mathrm{C}, \theta_{2}^{H}=65^{\circ} \mathrm{C}$, and $\theta_{2}^{L}=55^{\circ} \mathrm{C}$, and no module is specified as don't-care. These temperature limits are shown in Figure 5.2.1a with dashed/dotted lines.

A temperature simulation is performed based on a proper periodic schedule and the simulated temperatures are shown in Figure 5.2.1a. Starting from the ambient temperature $\left(\theta^{\text {ambient }}=30^{\circ} \mathrm{C}\right)$, the modules' temperatures steadily raise until they are inside the specified ranges. As shown in this example, applying heating sequences can drive the modules of an IC into a high temperature situation. For example, the temperature of module $m_{0}$ has reached $120^{\circ} \mathrm{C}$ at around $4 \times 10^{4}$ Time Units (TU). A TU consists of $4 \times 10^{7}$ test cycles in this example.

The temperatures around the $6 \times 10^{4} \mathrm{TU}$ point, are amplified and shown in Figure 5.2.1b. The time interval shown in Figure 5.2.1b corresponds to three periods of the schedule. Since the schedule is periodic, one period

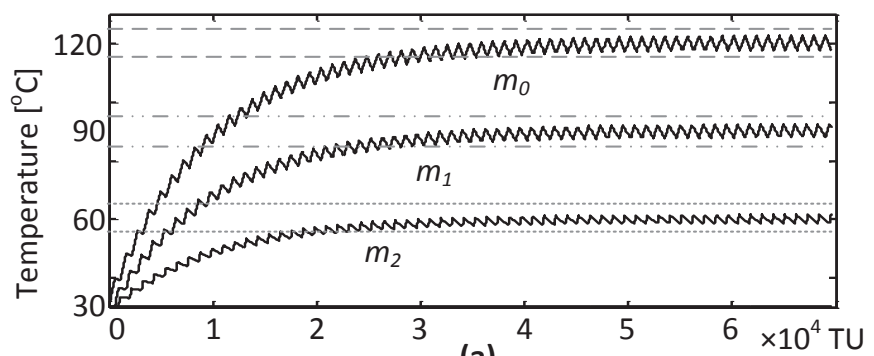

(a)

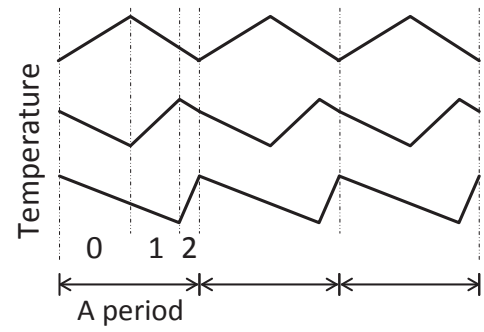

(b)

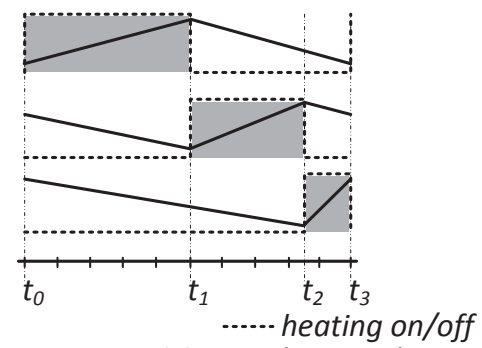

(c) - temperature

Figure 5.2.1 Temperature curves for an example 
captures the entire schedule which is repeated in a cyclic manner. Figure 5.2.1 $\mathrm{c}$ further amplifies and shows one period of the schedule that starts at $t_{0}$ and ends at $t_{3}$. The length of the period for this schedule is denoted by $\tau\left(\tau=t_{3}-t_{0}\right)$. One period is divided into three intervals, specified by numbers 0,1 , and 2 in Figure 5.2.1b.

They correspond to the time intervals ${ }^{3}\left[t_{0} t_{1}\right],\left[t_{1} t_{2}\right]$, and $\left[t_{2} t_{3}\right]$ in Figure 5.2.1c, respectively. The schedule specifies that the heating sequence for module $m_{0}$ is applied only in the $\left[\begin{array}{ll}t_{0} & t_{1}\end{array}\right]$ interval, the $\left[\begin{array}{l}\tau+t_{0} \\ \tau+t_{1}\end{array}\right]$

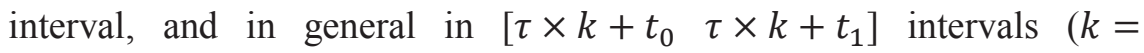
$0,1,2 \ldots)$, assuming that the process starts at time $t_{0}$. The application of the heating sequences for module $m_{1}$ and module $m_{2}$ are specified in a similar manner by the schedule.

For the $\left[\begin{array}{ll}t_{0} & t_{3}\end{array}\right]$ period, the time intervals that the heating sequences are applied are depicted by gray areas in Figure 5.2.1c. In this example, the TAM provides access to one module at a time $(W=1)$. Therefore, in interval $\left[t_{0} t_{1}\right]$ only module $m_{0}$ receives a heating sequence. Similarly, in $\left[t_{1} t_{2}\right]$ only $m_{1}$ is heated and the same goes for interval $\left[t_{2} t_{3}\right]$ for $m_{2}$. We need an efficient algorithm to generate such schedules.

\subsubsection{Steady State Solution}

Let us first analyze a simplified situation, where we assume that a steady state power could be provided for the modules. In this case, there exists a steady state solution that could generate and maintain the specified temperature map.

Providing continuous steady state powers simultaneously for all modules is, however, very likely to be impossible mainly due to TAM limitations. One solution is to use the maximal practical power for each core in combination with a Pulse Width Modulation (PWM) technique. Therefore, the best that can be achieved is a discrete stimulus sequence that has constant long-term average power with small ripples. This way, the modules have a time-divided multiple access to the TAM.

In order to reduce the risk of out of range temperatures due to ripples in the input power, the desired steady state temperatures are defined at the middle of the specified ranges $\theta_{m}^{S S}=\frac{1}{2} \times\left(\theta_{m}^{L}+\theta_{m}^{H}\right)$. Such ripples could be seen

\footnotetext{
${ }^{3}$ The notation $[\mathrm{a} b]$ is used to represent an interval that ranges from a to $b$.
} 
in the temperature curves given in Figure 5.2.1. In order to find the power values that result in the specified temperatures, the IC's temperature model should be analyzed.

As previously discussed, the temperature model works by dividing an IC into elements represented by nodes. Each node has a heat capacitance modelling its thermal capacity. Adjacent nodes are connected through a heat resistance that models the thermal conductivity between them. They are connected together in a network configuration, similar to an electric circuit. The temperatures correspond to voltages and the heat dissipation corresponds to a current source. A node is called active if it directly receives electrical power caused by switching activities.

A 3D-SIC is usually laid out so that the main blocks (e.g., logic and memory) are placed in a certain distance relative to TSVs to avoid undesirable effects induced by TSVs such as high mechanical stress. Such forbidden areas are called Keep-Out-Zones (KOZ) [Chakrabarty12, Deutsch12]. A collection of the TSVs placed next to each other (perhaps to overlap the KOZ of different TSVs and save area on the die) is called a TSV block. A TSV block may consist of only one TSV if the TSVs are placed far apart.

In this section (section 5.2.2) it is assumed that a module is a single active thermal node. Furthermore, it is assumed that TSV blocks are always thermally don't-care and do not dissipate heat (are passive thermal nodes) since their drivers are placed together with the corresponding modules. These assumptions will be relaxed in section 5.2.4. The temperature equation (equation 2.6.1) is repeated below for convenience:

$$
\boldsymbol{A} \times \frac{d}{d t} \boldsymbol{\Theta}+\boldsymbol{B} \times \boldsymbol{\Theta}=\boldsymbol{P}
$$

Like before, $\boldsymbol{\Theta}$ is the temperature vector and $\boldsymbol{P}$ is the power vector. Heat transfer among nodes is included in the temperature model and it means that a node can be heated up by its neighboring nodes even if it has no switching activities.

The specified temperature map consists, in fact, of the steady state temperatures that must be enforced on the IC for a while. A temperature map could be thought as the targeted steady state temperatures, $\boldsymbol{\Theta}^{S S}$, which are composed of the desired steady state temperatures for each module (e.g., $\theta_{m}^{S S}$ for module $m$ ). Since $\boldsymbol{\Theta}^{S S}$ is, in this case, equivalent to the steady 
state temperatures, which are considered constant (for a certain amount of time), its derivatives are zero (no variation in time). Therefore, equation 5.2.1 (similar to equation 3 in [Aghaee14b]) may be written as

$$
\boldsymbol{P}^{S S}=\boldsymbol{B} \times \boldsymbol{\Theta}^{S S}
$$

This means that it is possible to calculate the required powers that lead to the specified temperature map. In order for the specified temperature map to be achievable, the computed steady state power values must satisfy a feasibility and a schedulability condition. The first part of the feasibility condition is that the computed steady state power for module $m\left(p_{m}^{S S}\right)$ should be larger than or equal to the stray power dissipated by the module.

The stray power is an unintended part of the power that could not be independently controlled. Its value for module $m$ is denoted by $\overline{p_{m}}$. It consists of the leakage power in addition to the clock networks' power. As previously discussed, the clock networks' power can be large [Oberg03]. Therefore, it is important to take it into account.

The second part of the feasibility condition is that $p_{m}^{S S}$ should be less than or equal to the average power of the corresponding heating sequence, $p_{m}^{H S}$, plus $\overline{p_{m}}$. Therefore, the feasibility condition is:

$$
\forall m, \overline{p_{m}} \leq p_{m}^{S S} \leq\left(p_{m}^{H S}+\overline{p_{m}}\right)
$$

Usually the feasibility condition is easily met if the specified temperature map is realistic (e.g., the specified temperature is neither lower than the ambient nor larger than the achievable temperature). Assuming that equation 5.2.3 is satisfied, the schedulability condition which is related to the limited TAM bandwidth should be verified. The challenging problem here is to create the required average power values, $\boldsymbol{P}^{S S}$, using the available TAM bandwidth. This is done by selectively applying the heating sequences to the modules.

The continuous application of the heating sequence generates an average dynamic power equal to $p_{m}^{H S}$. The desired power values, $p_{m}^{S S}$, which are smaller than $p_{m}^{H S}+\overline{p_{m}}$, are created by applying the heating sequence, $p_{m}^{H S}$, for a fraction of a time period. The average power in a period should be made equal to the required steady state power. As mentioned before, this is done using a technique similar to PWM. The ratio of the duration of heating sequence application to the overall time period is therefore called Duty-cycle $\left(D_{m}\right)$ and its value is calculated using the following equation. 


$$
D_{m}=\frac{\left(P_{m}^{S S}-\overline{p_{m}}\right)}{p_{m}^{H S}}
$$

The duty-cycles might not be achievable if their values are relatively large and if the TAM does not provide sufficient bandwidth. For example, assume a design with two modules, with the duty-cycles $D_{0}=0.6$ and $D_{1}=0.8$. This means that in a period of time equal to 1 , we need access to module 0 for $60 \%$ of the time and access to module 1 for $80 \%$ of the time. Therefore, simultaneous access to more than one module $(0.6+0.8=1.4$ modules) is needed. This means that the TAM must provide simultaneous access to these two modules otherwise these duty-cycles are not schedulable and the specified temperature map cannot be enforced.

Note that $D_{m}$ can be divided into pieces; for example $D_{1}=0.8$ could be implemented by first applying the heating sequence for a duration equal to $D_{1,0}=0.3$ at the beginning of the period and then for a duration of $D_{1,1}=$ 0.5 at the end of the same period. The feasibility and schedulability conditions could be written together using the duty cycle concept as follows:

$$
\begin{gathered}
\forall m, 0 \leq D_{m} \leq 1, \text { and } \\
\sum_{m=0}^{M-1} D_{m} \leq W
\end{gathered}
$$

In fact, the first line in equation 5.2.5 is identical to the feasibility condition in equation 5.2.3, which is written here in terms of the duty cycles. The second line in equation 5.2.5 is the schedulability condition, where $W$ is the number of modules that can access the TAM simultaneously. Given a temperature map that satisfies both feasibility and schedulability conditions, it is relatively simple to develop a schedule to deliver the required duty cycles.

Figure 5.2.2a gives an illustrative example, where the available parallelism, $W$, provided by the TAM is represented by the number of rows that could be filled with duty-cycles, $D_{m} \mathrm{~s}(W=3)$. The scheduling algorithm starts by sorting the duty-cycles and then allocating them from the largest one to the smallest ones by filling the rows from the lowest one upwards. Note that if a duty-cycle starts in the lower row and continues to an upper row, it will not reach the end of the upper row, since $D_{m} \leq 1$.

Therefore, a duty cycle will, at most, be assigned to two TAM rows and a module needs to switch at most twice during a period. The overheads 
(a)

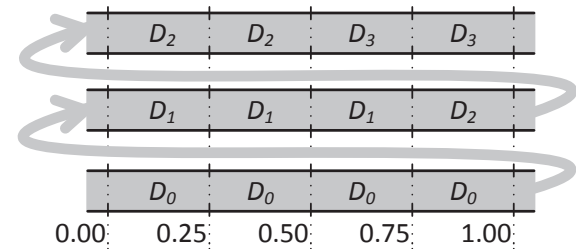

$W=3$ (three rows)

$M=4$ (four modules)

$m$ : module

$\mathrm{T}$ : the period

$D_{3}=0.50 \uparrow$
$D_{2}=0.75$ Sorted

$D_{1}=0.75$ Order

(b)

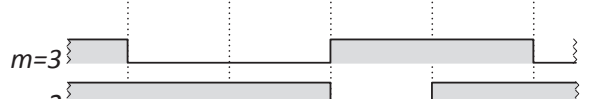

$D_{0}=1.00$

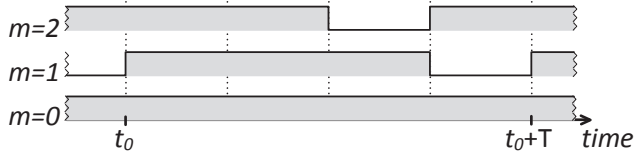

Figure 5.2.2 An example for scheduled duty-cycles

associated with switching are thus negligible. The fractions of the time period that the modules receive heating sequences are illustrated in Figure 5.2.2b. At every moment in time only three modules are receiving their heating sequences (the TAM limitation is not exceeded), and the average of applied heating sequence for a module in a period is equal to the specified steady state power.

As mentioned before, a thermal map may leave the temperatures for some nodes unspecified (don't-care nodes). Besides, the temperatures for inactive thermal nodes (e.g., TSV blocks) are also left unspecified. On the other hand, in order to compute the steady state powers, these temperatures should also be known. The proper choice of temperatures for the don'tcare nodes may help a thermal map that is otherwise not schedulable become schedulable. The problem of finding proper temperature values for the don't-care nodes could be formulated as a Linear Programming (LP) problem. Since we are more interested in knowing the duty cycles than the temperatures, the problem formulation is, then, written with the duty cycles as decision variables, as shown in Figure 5.2.3. The main objective is to find a feasible solution.

In Figure 5.2.3, the temperatures, $\theta_{m}$, should have the values specified by the thermal map, $\theta_{m}^{S S}$ (line 4 ). If not specified by the temperature map (e.g., don't care modules or inactive nodes) the temperatures should be between the ambient and the overheating temperature (line 5). For an inactive module, the power value should be equal to the stray power, $\overline{p_{m}}$, and therefore the duty cycles should be zero (line 6). For an active node, the duty cycles are between zero and one (line 7) according to equation 5.2.5. 
1. Decision variables: $\quad D_{m} ; m=0,1, \ldots, M-1$

2. Objective: find a feasible solution

3. Constraints:

4.

5.

$$
\left\{\begin{array}{l}
\theta_{m}=\theta_{m}^{S S}, \text { if } m \text { is specified } \\
\theta^{\text {ambient }} \leq \theta_{m}<\theta^{\text {over heating }}
\end{array}\right.
$$

6. $\quad\left\{D_{m}=0\right.$, if $m$ is inactive

7. $\quad\left\{0 \leq D_{m} \leq 1\right.$, if $m$ is active

8. $\quad \sum_{m} D_{m} \leq W ; m=0,1, \ldots, M-1$

9. Equations 5.2.2 and 5.2.4 relate variables $D_{m}$ and $\theta_{m}$.

\section{Figure 5.2.3 Linear programming formulation}

Besides, the duty cycles should satisfy the schedulability condition according to equation 5.2.5 (line 8 in Figure 5.2.3). The relation between the power values, $p_{m}^{S S}$, and the duty cycles is defined by equation 5.2.4. The temperatures, $\theta_{m}$, are computed based on power values, $p_{m}^{S S}$, using equation 5.2.2 (by replacing $\boldsymbol{P}^{S S}$ and $\boldsymbol{\Theta}^{S S}$ with vectors composed of $p_{m}^{S S}$ and $\theta_{m}$, respectively). If the LP solver finds a feasible solution, then the thermal map is achievable and the duty cycles are returned by the LP solver. We also have the temperature values of the don't-care modules. Knowing the duty cycles, a proper period for the PWM-like method has to be found.

\section{Finding an Appropriate Period for PWM-Based Schedule}

The duty cycles and the scheduling approach, discussed so far, are independent of the schedule's period, $\tau$. They generate the modules' temperatures such that their average equals the specified steady state temperatures. The period, $\tau$, should be short enough so that the fluctuations in the temperatures do not violate the specified limits $\left(\theta_{m}^{L}\right.$ and $\left.\theta_{m}^{H}\right)$. On the other hand, a longer period is desirable in order to minimize the switching actions in the schedule. An example for the results obtained by the proposed algorithm could be seen in Figure 5.2.1a. After the temperatures have completed their transitions to their new values (after $4 \times 10^{4} \mathrm{TU}$ ), the proper choice of the period keeps them inside the specified ranges, with a relatively low number of switching actions in the schedule.

In order to find a relatively long period, $\tau$, that albeit being long, keeps the temperature fluctuations inside the specified ranges, two different situations should be considered: ${ }^{(\mathrm{H})}$ heating sequence is applied (e.g., the second half of the period for module $m_{3}$ in Figure 5.2.2b); and ${ }^{(\mathrm{L})}$ no stimuli 


\section{Chapter 5}

are applied (e.g., the first half of the period for module $m_{3}$ in Figure 5.2.2b). In order to estimate the proper period for situation ${ }^{(\mathrm{H})}$, equation 5.2.1 is re-written around the steady state temperature for the heating sequence power, as shown in equation 5.2.6a. For situation ${ }^{(\mathrm{L})}$, equation 5.2.6b is used, instead.

$$
\begin{aligned}
\left(\frac{d}{d t} \boldsymbol{\Theta}\right)^{H} & =\boldsymbol{A}^{-1} \times\left(\boldsymbol{P}^{H S}+\overline{\boldsymbol{P}}-\boldsymbol{B} \times \boldsymbol{\Theta}^{S S}\right) \\
\left(\frac{d}{d t} \boldsymbol{\Theta}\right)^{L} & =\boldsymbol{A}^{-1} \times\left(\overline{\boldsymbol{P}}-\boldsymbol{B} \times \boldsymbol{\Theta}^{S S}\right)
\end{aligned}
$$

An illustrative example for the above equations is given in Figure 5.2.4. Equation 5.2.6a describes the tangent line that touches the temperature curve at point $\mathrm{A}$, around the steady state temperature. A similar example for equation 5.2.6b is the tangent line, $\mathrm{CD}$, in Figure 5.2.4. Equation 5.2.6a is then used to estimate the desired value for the period focusing only on the high temperature limit. Assume that the proper $\tau$, only focusing on situation ${ }^{(\mathrm{H})}$ and ignoring situation ${ }^{(\mathrm{L})}$, is denoted by $T_{m}^{H}$. Similarly, the proper $\tau$, only focusing on situation ${ }^{(\mathrm{L})}$, is denoted by $T_{m}^{L}$.

It is safe to assume that $D_{m} \times T_{m}^{H}$ ( $D_{m}$ is the duty cycle) is the amount of time that will result in a near violation situation for module $m$ in situation (H). In order to estimate $T_{m}^{H}$, first the derivative on the left side of equation 5.2.6a is linearly approximated as follows:

$$
\frac{\theta_{m}^{H}-\theta_{m}^{S S}}{D_{m} \times T_{m}^{H}} \cong\left(\frac{d}{d t} \boldsymbol{\Theta}\right)_{m}^{H} .
$$

Now, $T_{m}^{H}$ is computed for module $m$ as

$$
T_{m}^{H} \cong\left(\theta_{m}^{H}-\theta_{m}^{S S}\right) /\left(D_{m} \times\left(\frac{d}{d t} \boldsymbol{\Theta}\right)_{m}^{H}\right) .
$$

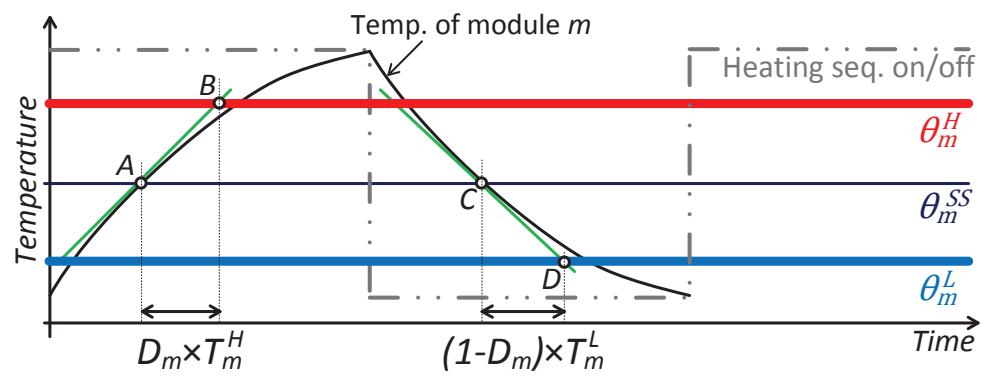

Figure 5.2.4 An example for the computation of a safe period

A long but safe period is computed so that the temperature limits will not be violated any more 
The values for $\left(\frac{d}{d t} \boldsymbol{\Theta}\right)_{m}^{H}$ are obtained from the right side of equation 5.2.6a and, consequently, the values for $T_{m}^{H}$ are computed using equation 5.2.8. For example, in Figure 5.2.4, when the module is receiving active power, the derivative that is represented by a straight line is tangential to the temperature curve at its intersection point with the steady state temperature at point $\mathrm{A}$ and later on intersects with the high temperature limit at point B. The period, $T_{m}^{H}$, is then calculated based on the time difference between $\mathrm{A}$ and $\mathrm{B}$. The other part of the line that stand between A and the low temperature limit is deliberately left out in order to achieve a shorter period that is safe in most of the situations (e.g., variation in the input power).

In a similar manner values for situation ${ }^{(\mathrm{L})}, T_{m}^{L}$, are calculated based on equation 5.2.6b focusing only on the low temperature limit. Since the temperatures should not violate any of the specified limits, the shortest $T_{m}$ $\left(T_{m}=\min \left\{T_{m}^{H}, T_{m}^{L}\right\}\right)$ is selected as the acceptable period for module $m$. The actual period, $\tau$, should be the smallest among the acceptable periods for all modules $\left(\tau=\min _{m}\left\{T_{m}\right\}\right)$ so that none of the temperature limits for the modules is violated. For example, after the temperatures have completed their transitions to their new values in Figure 5.2.1 (after $4 \times$ $10^{4}$ time units), the proper choice of the period keeps them insides the specified ranges, albeit relatively large fluctuations caused by relatively low number of switching actions in the schedule.

Moreover, the average of the applied heating sequences for each module is equal to the specified steady state power for it. For example in Figure 5.2.1c, modules $m_{0}, m_{1}$, and $m_{2}$ receive 50,35, and 15 percent of $p_{0}^{H S}$, $p_{1}^{H S}$, and $p_{2}^{H S}$ plus $\overline{p_{0}}, \overline{p_{1}}$, and $\overline{p_{2}}$, respectively. This is indicated by the width of the gray areas as compared with the schedule's period, $\tau(\tau=$ $\left.t_{3}-t_{0}\right)$.

\subsubsection{Transient Solution}

Up till now, it was assumed that the power values applied to an IC during transition to a new map are the same steady state powers that are used to maintain the new map afterwards. This implies that the transition to a new map is very slow and the transition time may be excessively long. For example, as shown in Figure 5.2.1, it takes about $4 \times 10^{4}$ time units for the IC to reach the specified thermal map from the ambient temperature. 
Here, in this section, burn-in time is the time required for bringing the IC into a thermal situation that complies with the first thermal map and then to the next map, until all maps are applied. It is likely that a large number of thermal maps are specified and therefore the transition to a new map should happen very fast. After transition, the map is maintained using the steady state powers, $\boldsymbol{P}^{S S}$, as calculated in the previous section. In order to reduce the burn-in time, a new solution that takes the transient response into account and uses larger or smaller power values (compared to the steady state solution) is presented here.

In this section, the transient response is taken into account while minimizing the overall transition time. We start by looking into the analytic solution for equation 5.2.1. This was previously discussed in section 4.6. The closed-form solution for a duration of time equal to $t$, is copied below from equation 4.6.5:

$$
\boldsymbol{\Theta}^{t}=\boldsymbol{\alpha}(t) \times \boldsymbol{\Theta}^{0}+\boldsymbol{\beta}(t) \times \boldsymbol{P}^{B}
$$

In the above equation, $\boldsymbol{\alpha}(t)$ and $\boldsymbol{\beta}(t)$ are matrices that are computed based on $\boldsymbol{A}$ and $\boldsymbol{B}$, and for a duration of time equal to $t$, as follows (similar to equation 4.6.3-4):

$$
\begin{gathered}
\boldsymbol{\alpha}(t)=\exp \left(-\boldsymbol{A}^{-1} \times \boldsymbol{B} \times t\right) \\
\boldsymbol{\beta}(t)=(\boldsymbol{I}-\boldsymbol{\alpha}(t)) \times \boldsymbol{B}^{-1}
\end{gathered}
$$

In the rest of this chapter $\boldsymbol{\alpha}(t)$ and $\boldsymbol{\beta}(t)$ are represented as $\boldsymbol{\alpha}$ and $\boldsymbol{\beta}$, respectively. The initial temperatures are expressed by $\boldsymbol{\Theta}^{0}$ and the temperatures at time $t$ is denoted by $\boldsymbol{\Theta}^{t}$. $\boldsymbol{P}^{B}$ is the power vector that is assumed to be constant for the time interval $t$. An intuitive explanation of equation 5.2.9 is that $\boldsymbol{\alpha}$ determines how fast the initial temperatures fade away and $\boldsymbol{\beta}$ determines how fast the input power affects the temperatures. As mentioned before, achieving a new temperature map in a short time is crucial and, therefore, this transition should happen as fast as possible. Once the IC's temperatures have converged to the specified temperature map, they can be maintained using the steady state powers, $\boldsymbol{P}^{S S}$, found by the steady state solution as presented in the previous section.

We would like to extend the steady state solution approach to equation 5.2.9, which includes the transient response, in order to find the 
schedulable power values that result in the shortest transition time. The new problem can be formulated as:

Find the shortest transition time, $t$, and the corresponding power values, $\boldsymbol{P}^{B}$, such that the specified map is achievable.

The transition time from map $\mu_{i}$ to map $\mu_{j}$ is defined as the time required to construct the temperatures specified by map $\mu_{j}$ starting from temperatures specified by map $\mu_{i}$.

This problem can be solved using an iterative approach that tries different alternatives for $t$. The main part of the proposed algorithm is illustrated in Figure 5.2.5. The algorithm uses the latest information regarding the interval that contains the optimal transition time. This interval is denoted by $[\lambda \sigma]$. At any step, it is known from the previous steps that the specified map is not achievable for transition times shorter than $\lambda$.

It is also known that since the temperature map is achievable for a transition time equal to $\sigma$, longer transition times are not optimal. Initially $\lambda$ is set to zero and $\sigma$ to the transition time for the steady state approach $\left(1^{\text {st }}\right.$ step in Figure 5.2.5). This steady state transition time is obtained by simulating the temperatures when the steady state schedule is used. A number of candidate transition times with uniform distances are selected between $\lambda$ and $\sigma$ ( $2^{\text {nd }}$ step in Figure 5.2.5) according to:

$$
t_{r}=\lambda+(\mathrm{r}+1) \times \frac{\sigma-\lambda}{R+1}, r=0,1, \ldots, R-1
$$

The $r$-th candidate transition time is denoted by $t_{r} . R$ is the number of parallel LP solvers and its value is selected based on the degree of parallelism offered by the platform that runs the algorithm. For example, for a machine that supports eight threads, eight is a reasonable choice for $R$. For each candidate $t_{r}$, solving the LP formulation determines whether the temperature map is achievable or not ( $3^{\text {rd }}$ step in Figure 5.2.5). This is represented by the Boolean variable, $\gamma_{r}$, for the $r$-th candidate transition time.

The value of $\sigma$ is updated to be equal to the smallest $t_{r}$ that leads to schedulable power values. The value of $\lambda$ is updated to be equal to the largest $t_{r}$ that leads to power values that are not schedulable $\left(4^{\text {th }}\right.$ step in Figure 5.2.5). Note that if for all the candidate transition times, denoted by $t_{r}$ in Figure 5.2.5 $(r=0,1, \ldots, R-1)$ the map is achievable, then $\lambda$ 


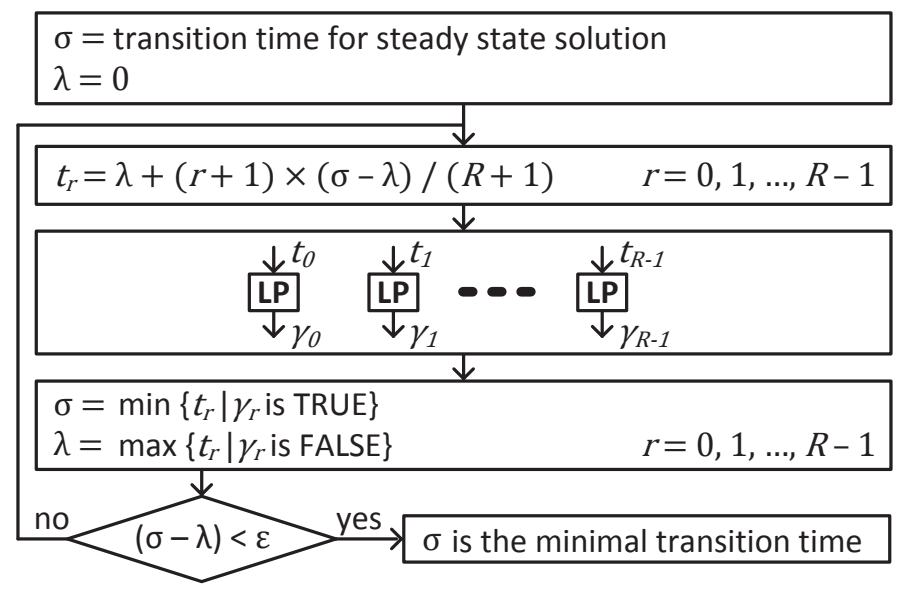

Figure 5.2.5 Main algorithm for the transient solution

remains unchanged. On the other extreme, if none of the $t_{r} \mathrm{~s}$ are schedulable then $\sigma$ remains unchanged. The algorithm stops when the smallest transition time is found with acceptably low error (i.e., $\varepsilon$ as shown in the conditional step in Figure 5.2.5). The error is bounded to $(\sigma-\lambda)$ and therefore if this difference is smaller than the specified limit, $\varepsilon$, then the actual error, too, will be smaller than $\varepsilon$.

The problem formulation for the LP solver that is used in the $3^{\text {rd }}$ step in Figure 5.2.5, is similar to the LP formulation in the previous section (Figure 5.2.3) with the following differences: (1) Instead of $\theta_{m} \mathrm{~s}$, the temperatures at the end of the transition time, $\theta_{m}^{t} \mathrm{~s}$, are used. (2) Instead of equation 5.2.2, equation 5.2.9 is used to calculate the temperatures based on the power values. The relation between the power values and the duty cycles defined by equation 5.2.4 is modified by replacing $\boldsymbol{P}^{S S}$ with $\boldsymbol{P}^{B}$ and used as indicated in line 9 in Figure 5.2.3. If the LP solver finds a feasible solution, the temperature map is achievable. This information is then used to update the $\lambda$ and $\sigma$ values.

Since during the transition the temperatures will not be in the specified ranges, the period for the PWM-like method is not crucial, unlike in the steady state solution. Therefore, it is sufficient that the period is much smaller than the transition time, $t$, so that the average power is a meaningful quantity for this span of time. For the experiments, the steadystate-solutions' periods are also used for the transient solution (they are much smaller than $t$ ). 
The matrix exponent computation for $\boldsymbol{\alpha}$, in equation 5.2.10, is performed using techniques proposed in [Ukhov12]. These techniques are used in order to speed up the repeated recalculations of $\boldsymbol{\alpha}$ and $\boldsymbol{\beta}$ for alternative transition times. They are based on eigenvalue decomposition, utilizing the inherent properties of matrices $\boldsymbol{A}$ and $\boldsymbol{B}$ and replace the excessively time consuming matrix exponent calculations in equation 5.2.10 with simpler operations. Although these techniques speed up the calculations, the required time is still very large, as experimentally shown in section 5.2.6.

Even though, the transient solution is an intuitive extension of the steady state solution and greatly outperforms it, it is slow in generating the schedules. Therefore, a new approach that avoids the time-consuming successive calculations of $\boldsymbol{\alpha}$ and $\boldsymbol{\beta}$ is necessary. Such an approach is proposed in the next section, based on a fast heuristic. Moreover, this new approach is capable of handling a more realistic problem formulation compared with the steady state and transient solutions.

\subsubsection{Transient-Based Heuristic}

So far, it has been assumed that it is possible to apply heating sequence to an arbitrarily selected active thermal node and, simultaneously, avoid application of heating sequences to all other nodes. This implies that the smallest element in the temperature model should not be smaller than the corresponding module on the TAM, in order to be able to control the heating sequence application to it independently from all other elements.

On the other hand, a temperature model with finer granularities might be preferable in order to achieve a better spatial precision in the simulated temperatures and perhaps the gradients. This way, the temperature maps can be planned with a higher resolution. Therefore, a technique that allows the modules to be further divided into a number of sub-modules is advantageous. These sub-modules correspond to a higher number of nodes in the temperature model.

Let us assume that the overall number of thermal nodes, denoted by $N$, is larger than or equal to the number of modules $(M \leq N)$. In the rest of this chapter, the desired temperature maps are specified for the thermal nodes instead of the modules. Consequently, the temperature map specifies that node $n$ has low temperature limit equal to $\theta_{n}^{L}$ and high temperature limit equal to $\theta_{n}^{H}(0 \leq n<N)$. 
In this new context, the switching activities for heating sequences are more specific and provide information concerning the power breakdown among active thermal nodes. For example, assuming that module $m$ is divided into two active thermal nodes $n$ and $o$, instead of only one heating sequence for module $m$, there will be two heating sequences corresponding to these two nodes.

The average power of a heating sequence for active node $n$ is represented by $p_{n}^{H S}$. The other active node of that module (i.e., node $o$ ) may also receive power, denoted by $p_{n, o}^{H S}$. Therefore, when trying to heat up node $n$ with $p_{n}^{H S}$, node $o$ is also heated by $p_{n, o}^{H S}$. Similarly, when trying to heat up node $o$ with $p_{o}^{H S}$, node $n$ is also heated by $p_{o, n}^{H S}$. Such a situation cannot be handled by the techniques previously proposed.

Furthermore, power dissipation for TSV blocks is now supported, and the TSV drivers/buffers may be placed in TSV blocks and their desired temperatures might also be specified in the temperature maps (not always don't-care, as assumed in the previous sections).

The proposed technique allows longer heating intervals during transition time as opposed to relatively shorter heating intervals during steady state (assuming that the new map's temperature is higher). This relatively long application of the heating sequence is called boosting. Boosting of an active node stops when the node reaches the Stop Boosting temperature, $\theta_{n}^{S B}$. The stop boosting temperature may be higher than the high temperature limit, $\theta_{n}^{H}$, but it is always lower than $\theta^{\text {overheating }}$.

Boosting is helpful in different ways. One way is to achieve the following desirable scenario. Assume that the node is initially heated beyond $\theta_{n}^{H}$ $\left(\theta_{n}^{S B}>\theta_{n}^{H}\right)$. Then the node does not need to receive heating sequence for a while and this leaves the TAM available for other nodes. Meanwhile, the temperature keeps decreasing naturally and just before the end of the transition time (the moment that all other nodes are in their specified temperature ranges), the temperature drops below the high temperature limit.

This simplifies and shortens the schedule for the transition period and, therefore, is desirable. An example for the temperature curves when the transient-based heuristic is used is given in Figure 5.2.6 for thermal node $n$. The overall transition time is indicated by the gray area. The temperature 
of node $n$ passes through the valid temperature range already in the interval (a) in Figure 5.2.6. But the termination of the transition interval is deferred since at least one of the other nodes, when $n$ is in the valid temperature range, is outside its valid range.

A node's temperature will naturally decrease if no power or little power is applied to it, but it should not fall below the low temperature limit. Therefore, a heating sequence should be applied at some point, before the temperature falls out of range. This point is marked with a temperature level named Heating Trigger and denoted by $\theta_{n}^{H T}$ for active thermal node $n\left(\theta_{n}^{H T}>\theta_{n}^{L}\right)$. The heating sequence should be applied when the temperature of node $n$ falls below $\theta_{n}^{H T}$.

The difference between $\theta_{n}^{H T}$ and $\theta_{n}^{L}$ provides sufficient time for the node to wait for gaining access to the TAM without its temperature falling below $\theta_{n}^{L}$. In Figure 5.2.6, the heating is required at the beginning of the interval (c), but since the TAM is not available, the node waits. At the beginning of the interval (d) the node has finally gained access to the TAM and the heating begins.

Heating should stop when the temperature reaches the high temperature limit. The time it takes to get back to the low temperature limit could be utilized to heat up other nodes that need heating. In a situation that a module consists of multiple active thermal nodes, the heating sequence could only be applied if all of these thermal nodes have temperatures lower than their high temperature limit.

The nodes that simultaneously require heating should be accommodated within the available bandwidth of the TAM. This bandwidth might not be sufficient for all of them and, therefore, the nodes that need heating more than others should be prioritized. The priorities for using the TAM are

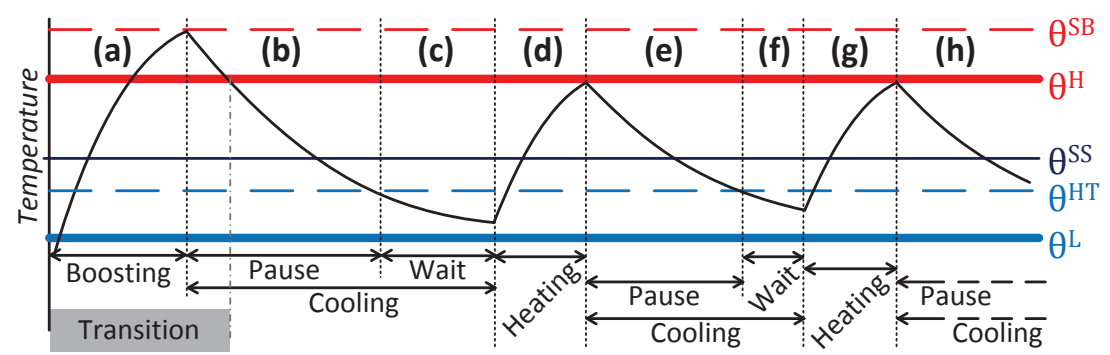

Figure 5.2.6 An example for transient-based heuristic 


\section{Chapter 5}

determined based on the regional need for heating (denoted by $d_{n}$ around a node $n$ ).

The value of $d_{n}$ is recomputed whenever node $n$ needs heating. A node requires heating in the following two situations: (1) When $\theta_{n}<\theta_{n}^{H T}$, after the transition, for example the interval (d) in Figure 5.2.6. (2) When $\theta_{n}<$ $\theta_{n}^{S B}$, during the transition, for example the interval (a) in Figure 5.2.6. In the following, we explain how to calculate $d_{n}$ for situation (1). Regional need for heating for situation (2) is obtained in a similar manner by replacing $\theta_{n}^{H T}$ with $\theta_{n}^{S B}$.

Equation 5.2.1 is re-written here with the approximate derivatives:

$$
\frac{\boldsymbol{A} \times\left(\boldsymbol{\Theta}^{H T}-\boldsymbol{\theta}\right)}{T}+\boldsymbol{B} \times \boldsymbol{\Theta}=\overline{\boldsymbol{P}}+\boldsymbol{D} \times \boldsymbol{P}^{H S}
$$

The input power, $\boldsymbol{P}$, in equation 5.2.1 is substituted with the stray power, $\overline{\boldsymbol{P}}$, plus the PWM power of heating sequences, $\boldsymbol{D} \times \boldsymbol{P}^{H S}$. Vector $\boldsymbol{D}$ is the vector form of the regional need for heating and consists of $d_{n} \mathrm{~s}$. Equation 5.2.12 is written for one test cycle with period $T$ which is a very short time. The equation is then solved for the nodes that need heating as follows.

$$
d_{n}=\frac{\frac{\sum_{k=0}^{N-1} a_{n, k} \times\left(\theta_{k}^{H T}-\theta_{k}\right)}{T}+\sum_{k=0}^{N-1} b_{n, k} \times \theta_{k}-\overline{p_{n}}}{p_{n}^{H S}}
$$

The regional need for heating, $d_{n}$, depends on the required heating for node $n$ (consider the summations when $k$ is equal to $n$ ), on the required heating that is related to the adjacent nodes (consider the summations when $k$ denotes an adjacent node to $n$ ), and on the average power of the corresponding heating sequence, $p_{n}^{H S}$.

The regional need for heating for a node has the highest dependency on the node itself, and then a relatively high dependency on the adjacent nodes (this characteristic is captured by the temperature model). The influence of other nodes located far away from the targeted node is small. The heat transfer between nodes is taken into account automatically, since equation 5.2.13 is derived from the temperature equation (equation 5.2.1) and includes the thermal conductances from matrix $\boldsymbol{B}$. This is reflected by $b_{n, k}$ in equation 5.2.13.

Equation 5.2.13 ensures that the priority for using the TAM is given to the regions that need longer heating times, for example because of large 
$\left(\theta_{n}^{H T}-\theta_{n}\right)$ and small $p_{n}^{H S}$. Furthermore, the locality of this heuristic is helpful because adjacent nodes are likely to be in the same module and therefore these nodes will receive some desirable active heating power $\left(p_{n, k}^{H S}\right)$ or heat transferred from module $n$.

The problem with heat transfer exists also in the previous sections, but it was taken care of automatically by the LP solver. An effect of the interplay between priorities could be seen in Figure 5.2.6. The waiting period in the interval (f) is much shorter than the waiting period in the interval (c). The length of a waiting period depends on the other nodes' priorities in addition to the node $n$ 's priority. The priorities in thermal boost mode are computed in a similar manner by replacing $\theta_{n}^{H T}$ with $\theta_{n}^{S B}$ (e.g., in equation 5.2.1213).

As discussed before, the performance of the transient-based heuristic strongly depends on the stop boosting, $\theta_{n}^{S B}$, and heating trigger, $\theta_{n}^{H T}$, temperatures. One example is the priorities calculated using equation 5.2.13, since they depend on $\theta_{n}^{H T}$ after the transition and on $\theta_{n}^{S B}$ during the transition. Efficient values for these temperature levels for each temperature map and each thermal node are found using a PSO technique, as introduced in section 2.7.

\subsubsection{Remarks}

The output for the steady state and transient solutions is a periodic offline schedule and therefore producing a small periodic schedule is one of their advantages. The transient solution, on the other hand, returns also the transition time as an output. The periodic schedule generated by the transient solution is applied just during the transition time and then the steady state schedule must be used. A periodic schedule means that there is a constant average power for each module during the transition, despite the fact that a higher or lower average power might be suitable for different periods. The transient-based heuristic addresses this issue by generating a non-periodic offline schedule that facilitates the heating for the nodes that need it the most. Furthermore, the introduction of the boost mode helps to reduce the switching overheads in the schedule. For these reasons, the transient-based heuristic offers a reduced transition time.

The proposed approaches support also heating sequences generated by a Built-In Self-Test (BIST) engine. An example for the use of BIST engines during burn-in in order to achieve high toggle coverage is reported in 
[Carbine97]. Such BIST engines that stimulate high switching activities in a certain area of the IC under burn-in can be used to produce heating sequences online. The only difference, in our context, is that if the BIST engine does not occupy TAM, then it can be scheduled at any time as needed.

For instance assuming that module $m_{k}$ can receive its heating sequence from an adjacent BIST engine that is not occupying TAM, the $8^{\text {th }}$ line for the LP formulation in Figure 5.2.3 should be changed to: $\left(\sum_{m=0}^{k-1} D_{m}+\right.$ $\left.\sum_{m=k+1}^{M-1} D_{m}\right) \leq W$. The situation for the transient-based heuristic is even simpler, since the algorithm only needs to know that module $m_{k}$ can receive its heating sequence at any time. Then, $m_{k}$ does not need to compete with other modules for access to TAM. Consequently, there is no need to evaluate the regional need for heating for $m_{k}$.

The techniques proposed above make it possible to perform burn-in based on heating sequences without requiring a heat chamber. One of the situations when a heat chamber might be required is for the ICs that are designed to work in an extremely high temperature environment. For example, a microcontroller for a car engine is designed with low power in order not to raise too much its temperature from the very high ambient temperature in the engine area. When such a chip is tested or operated with regular low ambient temperature, it is impossible to have enough power density to boost its temperature to its usual high level in normal working condition.

Another such situation is when some parts of an IC (e.g., package pins, die to pin connections, and the interposer) cannot be heated up sufficiently by input stimuli. In such cases, an extremely hot burn-in condition might be required that is not achievable by exclusive use of heating sequences. Even in such cases the use of the methods proposed in this thesis for enforcing the temperature gradients will still be useful. The proposed algorithms do not need any modifications to work under such situations, except for setting a large ambient temperature corresponding to the heat chamber temperature. Note that as discussed previously in section 4.6 the thermal behavior is modeled as a Linear Time Invariant (LTI) system. Therefore, a larger ambient temperature will directly add up to the temperatures created by the application of the heating sequences. 
The focus of this chapter is not on the issues related to process variations. Small temperature variations can be tolerated by introducing a safety margin for the specified temperature limits, in particular the overheating temperature. Large temperature variations need a variation-aware technique, for example, by combining the method proposed in this chapter with the techniques proposed in the previous chapter. This is, however, outside the scope of this thesis.

We use the term "temperature gradients" to precisely refer to the spatial temperature differences. But we also use it in a relaxed manner to refer to different sites' temperature values. For example the temperature difference between two adjacent modules $m_{0}$ and $m_{1}$ that is $\theta_{1}-\theta_{0}$, is exactly a temperature gradient and speeds up the early life-time of the affected area. However, the fact that module $m_{0}$ 's temperature is equal to $\theta_{0}$ and $m_{1}$ 's is $\theta_{1}$ is not directly a temperature gradient. These facts are captured by a temperature map and affect the signal delays (for signals that are routed through or close to these modules).

\subsubsection{Experimental Results}

The proposed techniques are evaluated for twelve experimental ICs with one to three layers as detailed in Table 5.2.1, columns 2, 3, and 4. The onelayer experimental ICs (row 1 to 4) are bare dies and could represent the pre-bond test stage. The ICs that have two layers (row 5 to 8 ) could represent mid-bond test stage. The ICs with three layers (row 9 to 12) could represent post-bond test stage.

There are two, four, eight, and 16 physical modules per layer for different dies, resulting in the total number of modules ranging from two to 48 , as given in column 3 . There are one, two, and three TSV blocks per layer on the dies, resulting in the total number of TSV blocks given in column 4, ranging from one to nine. Each TSV block hosts a relatively large number of TSVs. The dies are assumed to be stacked in a face to back configuration.

The temperature models are extracted using an approach similar to the method proposed in [Coskun09] for 3D-SIC. This is an extended form of the technique used by HotSpot [Huang07] for normal 2D ICs. The heating patterns' switching activities are generated using Markov chains, similarly as in [Yao11c]. The temperature maps specify the valid temperature ranges for nodes in the temperature model. The valid ranges are randomly selected 
Table 5.2.1 Percentage changes achieved by proposed techniques

\begin{tabular}{|c|c|c|c|c|c|}
\hline $\begin{array}{l}\text { IC } \\
\text { Number }\end{array}$ & IC Specifica & tions & & $\begin{array}{l}\text { Percent } \\
\text { overall t }\end{array}$ & $\begin{array}{l}\text { ge change in } \\
\text { ransition time }\end{array}$ \\
\hline & $\begin{array}{l}\text { Number of } \\
\text { layers }\end{array}$ & $\begin{array}{l}\text { Number of } \\
\text { modules }\end{array}$ & $\begin{array}{l}\text { Number of } \\
\text { TSV blocks }\end{array}$ & $\begin{array}{l}\text { Transient } \\
\text { solution }\end{array}$ & $\begin{array}{l}\text { Transient- } \\
\text { based heuristic }\end{array}$ \\
\hline 1 & 1 & 2 & 1 & -83.88 & -97.82 \\
\hline 2 & 1 & 4 & 1 & -68.35 & -73.05 \\
\hline 3 & 1 & 8 & 2 & -64.97 & -69.95 \\
\hline 4 & 1 & 16 & 3 & -56.93 & -62.63 \\
\hline 5 & 2 & 4 & 2 & -64.37 & -68.37 \\
\hline 6 & 2 & 8 & 2 & -58.32 & -65.94 \\
\hline 7 & 2 & 16 & 4 & -57.19 & -63.82 \\
\hline 8 & 2 & 32 & 6 & -43.99 & -55.14 \\
\hline 9 & 3 & 6 & 3 & -70.44 & -97.18 \\
\hline 10 & 3 & 12 & 3 & -57.15 & -93.17 \\
\hline 11 & 3 & 24 & 6 & -84.56 & -95.87 \\
\hline 12 & 3 & 48 & 9 & -92.06 & -94.52 \\
\hline Average & & & & -66.85 & -78.12 \\
\hline
\end{tabular}

between $35-95^{\circ} \mathrm{C}$, and some modules/nodes are randomly selected to be don't-care.

Only temperature maps that can be achieved in practice are considered. An example for a temperature map that cannot be achieved is one that requires a central node with very low temperature and its adjacent nodes with very high temperature. In this case the temperature gradient is huge and it probably will require negative power (active cooling) for the central node.

The transient solution (section 5.2.3) and the transient-based heuristic (section 5.2.4) are evaluated and compared with the steady state solution (section 5.2.2). The transient-based method is capable of handling temperature models having multiple nodes per module, while the steady state and transient solutions only support one thermal node per module. In order to have comparable experiments, the temperature model that is supported by the steady state method is used for the other techniques.

The CPU time to generate the schedules for the transient-based method for all of the twelve experimental ICs together is about 12 minutes while the transient solution takes 17 minutes and steady state method completes in 2 seconds. As discussed earlier, the time required to bring the IC into a thermal situation that complies with the first temperature map and then to the next map until all maps are applied is defined as the overall transition time in this work. 
The percentage change in overall transition time offered by the transient solution and the transient-based heuristic, compared with the steady state solution, are given in columns 5 and 6 of Table 5.2.1, respectively. Considerable speed up ( $78 \%$ in average) is achieved by the transient-based heuristic and moreover, it also outperforms the transient solution.

The CPU times for the transient-based heuristic for different number of modules are given in Figure 5.2.7. Even though they grow rapidly with the increase in the number of modules, for an IC with 48 modules it is still relatively short $(480 \mathrm{sec})$.

\subsection{Temperature-Gradient Based Test}

For the temperature-gradient based test, the goal is to make sure that the tests are performed when the temperature gradients are correctly captured on the IC. This means that the specified temperature maps should be reached and maintained during the corresponding test periods. In the followings a straightforward algorithm and then a fast heuristic are proposed.

\subsubsection{Straightforward Algorithm}

This algorithm works by changing between two modes, the temperature construction mode and the test mode. Initially the temperature construction mode is activated and it creates the specified temperature map using a method similar to the transient-based heuristic proposed in section 5.2.4. Then the test mode is activated and the tests that are scheduled with a third party algorithm (e.g., scheduling method proposed in [SenGupta12]) are applied. The test temperatures are simulated at design time and as soon as at least one of the thermal nodes is out of its specified range, the test mode is paused and the temperature construction mode takes over again. When

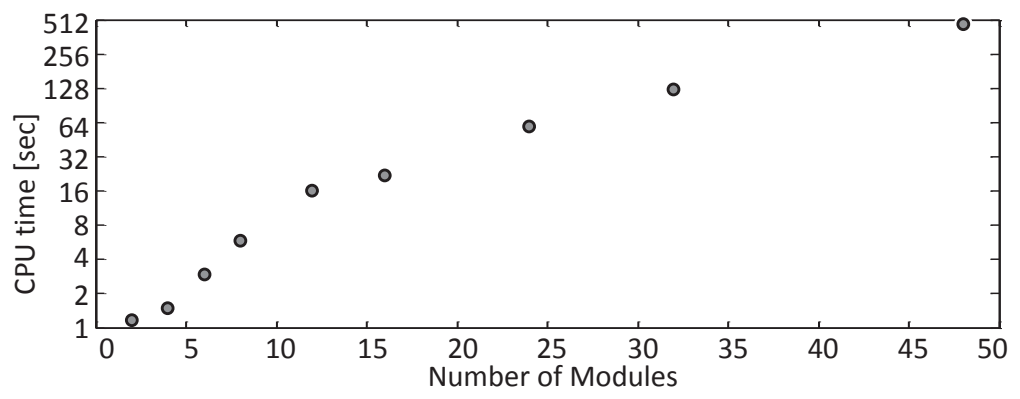

Figure 5.2.7 CPU time versus number of modules 
all thermal nodes are brought back into the specified temperature ranges, the temperature construction mode is paused and testing resumes.

Similar to the transient-based heuristic, if the temperature of a node is lower than the heating trigger temperature, it should be heated by applying the heating sequence to it. If there are many nodes that need heating (more than what the TAM can support), priority is given to those with higher regional need for heating as defined in section 5.2.4. The construction mode, unlike the transient-based heuristic, should not heat the nodes up to their high temperature limit since the power of the tests that are applied immediately after the construction mode may rapidly heat up the node beyond high temperature limit. Therefore, Testing Trigger temperatures which are denoted by $\theta_{n}^{T T}$ for node $n\left(\theta_{n}^{H T}<\theta_{n}^{T T}<\theta_{n}^{H}\right)$ are introduced here. During the temperature construction mode, the heating for node $n$ stops as soon as the temperature reaches $\theta_{n}^{T T}$.

In the test mode, as soon as the temperature of a node reaches the high temperature limit, the test mode is immediately paused, the temperature construction mode is activated and, consequently, a cooling interval is applied. The cooling continues until the node is cooled down to the testing trigger temperature, $\theta_{n}^{T T}$, and then the node is ready for testing again. The actual activation of the test mode will also depend on the temperatures of the other nodes. Efficient values for testing trigger temperatures, $\theta_{n}^{T T}$, for each map are found using a particle swarm optimization technique along with $\theta_{n}^{S B}$ and $\theta_{n}^{H T}$.

The inputs to the methods proposed here in section 5.3 include the inputs to the methods proposed in section 5.2 in addition to the test specifications (e.g., test switching activities). The output is a set of offline schedules. Moreover, the proper values for the heating trigger, $\theta_{n}^{H T}$, stop boosting temperatures, $\theta_{n}^{S B}$, and testing trigger temperatures, $\theta_{n}^{T T}$, which result in a rapid test could also be considered as the outputs that provide a basis for an online scheduling scenario.

The straightforward algorithm is simple, and allows the choice of a desired arbitrary test schedule that is used in the test mode. But the overall test application time offered by this method is very long. Note that the total test application time also includes time intervals spent for temperature construction 


\subsubsection{Fast Heuristic}

The fast heuristic schedules the tests together with the heating sequences such that the specified temperature map is maintained. This way, a shorter test application time can be achieved. An illustrative example for the proposed method is given in Figure 5.3.1 for a single thermal node. The proposed technique has similarities to the temperature construction algorithm in section 5.2.4. For example, stop boosting temperature, $\theta_{n}^{S B}$, indicates that the boosting should stop, as illustrated at the end of interval (a) in Figure 5.3.1. After being too warm, the node should cool until its temperature gets below the testing trigger temperature, $\theta_{n}^{T T}$, as shown in interval (b).

When the temperatures for all of the other thermal nodes covered by module $m$ are between their high temperature limit, $\theta_{n}^{H}$, and their heating trigger, $\theta_{n}^{H T}\left(\theta_{n}^{H}>\theta_{n}^{H T}\right)$, testing may start, as in interval (c) in Figure 5.3.1. All other nodes should be within their temperature limits $\left[\theta_{n}^{L} \theta_{n}^{H}\right]$. Testing continues until the temperature of at least one of the nodes goes beyond the high temperature limit $\theta_{n}^{H}$ or falls below the heating trigger $\theta_{n}^{H T}$. For example at the end of interval (c), the node is too cold for testing and a heating interval should be introduced. Note that the TAM may no longer be available and, therefore, the node is waiting for access to the TAM in interval (d).

Finally, when access to the TAM is obtained, the heating sequence is applied in interval (e). In order to start heating, all nodes covered by a module should be colder than the high temperature limit since the heating sequence for one node is very likely to inject power to other nodes in the same module (as explained in section 5.2.4). Heating continues until the temperature goes beyond the testing trigger temperature and, then, the test resumes as in interval (f) in Figure 5.3.1. When the temperature reaches the high temperature limit, a cooling interval is introduced as in interval (g).

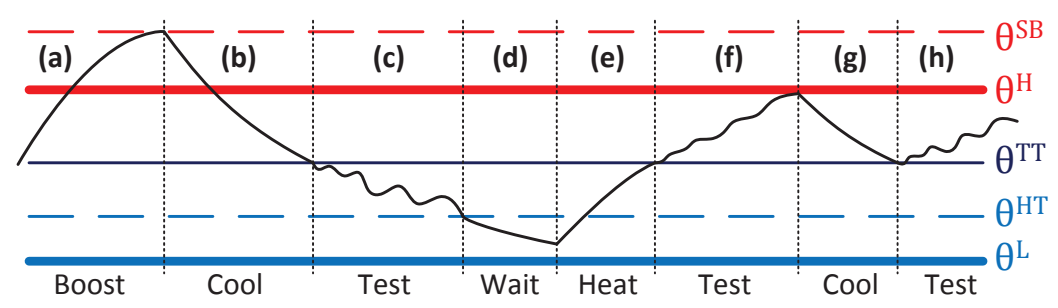

Figure 5.3.1 An example for the fast heuristic 


\section{Chapter 5}

This procedure continues until all tests corresponding to the current temperature map are completed.

As mentioned before, nodes will compete for access to the TAM and, therefore, some of them should be prioritized. First the nodes that require heating (not the tests) are granted access to TAM. This helps to keep the temperatures most of the time within the specified limits and, thus, keep the flow of the tests uninterrupted. Note that if only one node falls out of its specified range, all tests must be interrupted until the map is achieved again. This will waste a lot of time, since the tests for the modules that are in their specified range should also be interrupted. The priorities for the nodes that require heating are determined based on the regional need for heating as proposed in section 5.2.4 (equation 5.2.13).

If the TAM is left with some available bandwidth after the heating sequences are scheduled, the modules that are thermally qualified may resume their tests. A module is thermally qualified if none of the nodes that correspond to that module are demanded by the previously discussed rules to receive heating, wait for heating, or receive cooling. The priority is given to the modules that are expected to offer long test endurance. The test endurance is denoted by $e_{m}$ for module $m$, and is defined as:

$$
e_{m}=t t_{m} \times r_{m}
$$

The test endurance is directly proportional with the remaining test size denoted by $r_{m}$ for module $m$. The larger the remaining test size, the longer the test endurance. The thermal tolerance, denoted by $t t_{m}$ for module $m$, is the other contributor to the test endurance. High thermal tolerance, $t t_{m}$, indicates that the module is capable of receiving tests for a relatively long time without exceeding the specified thermal limits. Therefore, a module with large thermal tolerance may remain under test for a relatively long time. The thermal tolerance is defined as:

$$
t t_{m}=\min _{k}\left\{\Delta_{k}\right\}
$$

In equation 5.3.2, it is assumed that module $m$ covers $K$ active thermal nodes. $\Delta_{k}(k=0,1, \ldots, K-1)$ denotes the expected thermal distance to a temperature limit for node $k$ and is defined as:

$$
\Delta_{k}=\left\{\begin{array}{c}
\theta_{k}^{H}-\theta_{k}, \text { if upcoming_tests_power }>p_{k}^{S S} \\
\theta_{k}-\theta_{k}^{H T}, \text { if upcoming_tests_power } \leq p_{k}^{S S}
\end{array}\right.
$$


As mentioned in section 5.2.2, the desired steady state power $p_{k}^{S S}$ is the power that results in a temperature equal to $\theta_{n}^{S S}=\frac{1}{2} \times\left(\theta_{n}^{L}+\theta_{n}^{H}\right)$. Equation 5.3.3 indicates that if the upcoming tests have relatively high average power, then it is likely that the thermal node exceeds the high temperature limit and, therefore, the difference between the current temperature, $\theta_{k}$, and the high temperature limit, $\theta_{k}^{H}$, is a good measure for thermal tolerance.

Similarly, for a relatively low power test, it is more likely that the temperature falls below the heating trigger in the future. Therefore, the difference between the current temperature, $\theta_{k}$, and the heating trigger temperature, $\theta_{k}^{H T}$, is a good measure for thermal tolerance. Thermal tolerance, $t t_{m}$, is defined as the smallest $\Delta_{k}(k=0,1, \ldots, K-1)$ since as soon as a single node is out of the specified range $\left[\theta_{n}^{L} \theta_{n}^{H}\right]$, disregarding of the temperatures of the other nodes, test should be interrupted. Note that if the temperature falls below $\theta_{n}^{H T}$, only for a node in module $m$, then the test is interrupted only for module $m$.

A proper value for the testing trigger temperature, $\theta_{n}^{T T}$ is selected so that the temperature variation during test (caused by the variations in the test power) rarely results in the temperatures below $\theta_{n}^{H T}$ or above $\theta_{n}^{H}$. Every time that $\theta_{n}^{H T}$ or $\theta_{n}^{H}$ are violated, the test must be interrupted and a heating or cooling interval must be introduced, respectively. Since these are time consuming, a proper $\theta_{n}^{T T}$ value helps to obtain a short test application time by reducing the number of interruptions. Besides the testing trigger temperature, stop boosting and heating trigger temperatures $\left(\theta_{n}^{S B}\right.$ and $\theta_{n}^{H T}$ respectively) have a considerable effect on the test application time and therefor proper values for them should be found. A particle swarm optimization technique, as discussed in section 2.7, is used to find the proper values for $\theta_{n}^{S B}, \theta_{n}^{H T}$, and $\theta_{n}^{T T}$ for each map.

\subsubsection{Experimental Results}

The fast heuristic (section 5.3.2) is evaluated and compared with the straightforward method (section 5.3.1). An experimental setup similar to section 5.2.6 is used here. This includes experimental ICs described in Table 5.2.1. For convenience, columns 1-4 from this table are repeated in Table 5.3.1 that reports the experimental results. The temperature model used for these experiments has multiple nodes per module, as opposed to experiments presented in section 5.2.6. 
The total time required to enforce a temperature map and maintain it while the tests are being applied, in addition to the time spent applying the corresponding tests, is defined as the test time in this section. The percentage change in test time offered by the fast heuristic compared with the straightforward method is given in column 5 of Table 5.3.1, which shows that considerable speed up ( $67 \%$ in average) is achieved.

The percentage change in CPU time required by the fast heuristic compared with the straightforward method is $-36 \%$. The overall CPU time depends on the interaction between the computational complexity of a single decision point ${ }^{4}$ in the schedule and the schedule length. The experimental results indicate that since the fast heuristic method makes better decisions, compared with the straightforward method, the overall length of the schedule is reduced considerably and therefore the overall CPU time is also reduced. This happens despite of the fast heuristic's higher computational complexity for individual decision points. In fact, the schedule length is an important contributor to the CPU time, since longer schedules require longer temperature simulations and temperature simulation is, per se, very time consuming.

Table 5.3.1 Percentage changes achieved by fast heuristic

\begin{tabular}{|c|c|c|c|c|}
\hline \multirow{2}{*}{$\begin{array}{l}\text { IC } \\
\text { Number }\end{array}$} & \multicolumn{3}{|c|}{ IC Specifications } & \multirow{2}{*}{$\begin{array}{l}\text { Percentage change in } \\
\text { test time achieved by } \\
\text { fast heuristic }\end{array}$} \\
\hline & $\begin{array}{l}\text { Number of } \\
\text { layers }\end{array}$ & $\begin{array}{l}\text { Number of } \\
\text { modules }\end{array}$ & $\begin{array}{l}\text { Number of } \\
\text { TSV blocks }\end{array}$ & \\
\hline 1 & 1 & 2 & 1 & -16.97 \\
\hline 2 & 1 & 4 & 1 & -39.69 \\
\hline 3 & 1 & 8 & 2 & -63.35 \\
\hline 4 & 1 & 16 & 3 & -94.77 \\
\hline 5 & 2 & 4 & 2 & -8.70 \\
\hline 6 & 2 & 8 & 2 & -60.80 \\
\hline 7 & 2 & 16 & 4 & -78.17 \\
\hline 8 & 2 & 32 & 6 & -95.04 \\
\hline 9 & 3 & 6 & 3 & -75.90 \\
\hline 10 & 3 & 12 & 3 & -84.81 \\
\hline 11 & 3 & 24 & 6 & -87.08 \\
\hline 12 & 3 & 48 & 9 & -94.72 \\
\hline Average & & & & -66.67 \\
\hline
\end{tabular}

\footnotetext{
${ }^{4} \mathrm{~A}$ decision point is a point in the schedule where the scheduling algorithm must decide about the upcoming states (e.g., whether to cool, wait, heat, or test).
} 
The CPU times for the fast heuristic for different number of modules are given in Figure 5.3.2. Even though they grow rapidly with the increase in the number of modules, for an IC with 48 modules it is still acceptably short. The CPU times for the burn-in (section 5.2) will be relatively shorter since here the tests are also scheduled along with the heating sequences. The increase rate in the CPU times, as shown in Figure 5.3.2, is tolerable similar to the transient-based heuristic (section 5.2.4). This was expected since these algorithms are very similar.

\subsection{Temperature-Map Ordering}

The order in which the maps are enforced has a considerable impact on the overall burn-in and test time. Since there are usually a number of temperature maps to be applied, their ordering is important. In this section we present methods to rapidly obtain a proper order for temperature maps that results in a short burn-in and test time.

\subsubsection{Map Ordering Technique}

To simplify the discussions, let us assume that the temperature map for a thermal node is represented by the middle value of the specified temperature range $\theta_{n}^{S S}=\frac{1}{2} \times\left(\theta_{n}^{L}+\theta_{n}^{H}\right)$. As an example, assume that an IC has two thermal nodes and the initial temperature is $30^{\circ} \mathrm{C}$. The specified temperatures, by temperature map $\mu_{0}$, are denoted by $\left\{\theta_{0}^{S S}, \theta_{1}^{S S}\right\}^{5}$. This means that temperatures $\theta_{0}^{S S}$ and $\theta_{1}^{S S}$ are specified by map $\mu_{0}$ for nodes $n_{0}$ and $n_{1}$, respectively. Assume that there are three temperature maps

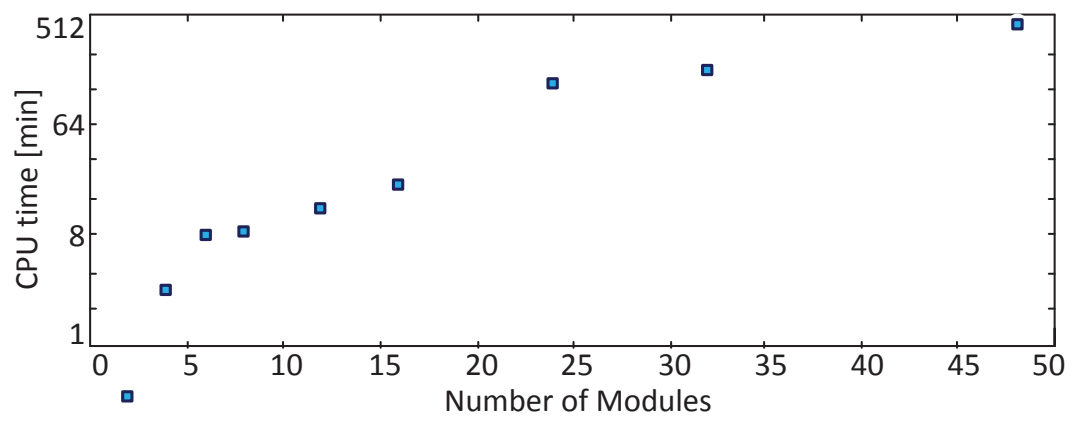

Figure 5.3.2 CPU time versus number of modules

${ }^{5}$ The notation $\left\{a_{0}, a_{1}, \ldots, a_{K}\right\}$ is used to represent an ordered sequence of elements $a_{k}(k=0,1, \ldots, K)$. 
denoted by $\mu_{0}, \mu_{1}$, and $\mu_{2}$. These maps specify the following temperatures: $\mu_{0}=\left\{110^{\circ} \mathrm{C}, 90^{\circ} \mathrm{C}\right\}, \mu_{1}=\left\{40^{\circ} \mathrm{C}, 50^{\circ} \mathrm{C}\right\}$, and $\mu_{2}=\left\{110^{\circ} \mathrm{C}, 80^{\circ} \mathrm{C}\right\}$, respectively.

These temperature maps are represented in Figure 5.4.1a-b by three points in a Cartesian space. The temperature for node $n_{0}$ is represented by the horizontal axes, $\theta_{0}$, and for node $n_{1}$ by the vertical axes, $\theta_{1}$. The initial order of temperature maps $\left\{\mu_{0}, \mu_{1}, \mu_{2}\right\}$ requires a long time to increase the temperature for node $n_{0}$ from 30 to 110 ( $a_{0}$ in Figure 5.4.1a), then decrease it to 40 ( $a_{1}$ in Figure 5.4.1a), and then again increase it from 40 to $110\left(a_{2}\right.$ in Figure 5.4.1a). This process will take a long time due to the required large changes in the temperature. In contrast, it is much faster to work with the maps ordered as $\left\{\mu_{1}, \mu_{2}, \mu_{0}\right\}$, since in this case, the required temperature changes consist of smaller temperature variations, as shown in Figure 5.4.1b.

As discussed earlier, in order to minimize the overall transition time for burn in, a particle swarm optimization technique finds the proper values for stop boosting and heating trigger temperatures $\left(\theta_{n}^{S B} \mathrm{~S}\right.$ and $\theta_{n}^{H T} \mathrm{~s}$, respectively). The map orders should be optimized along with these temperatures, since all of these factors have a crucial effect on the overall transition time for a given set of temperature maps. The naïve approach to find proper map orders is to introduce them as decision variables into the PSO along with $\theta_{n}^{S B} \mathrm{~S}$ and $\theta_{n}^{H T} \mathrm{~s}$. Experiments showed that this naïve approach takes very long CPU time to complete. Since the optimized values for $\theta_{n}^{S B}$ and $\theta_{n}^{H T}$ depend on the map order, different map orders result in different optimized values for $\theta_{n}^{S B}$ and $\theta_{n}^{H T}$.

The initial PSO population in the naïve approach consists only of random solutions (random $\theta_{n}^{S B} \mathrm{~s}, \theta_{n}^{H T} \mathrm{~s}$, and random map orders). Introducing a relatively good map order into the initial population of PSO (among other initial solutions that are random) will help to speed up the search. This approach is denoted by A1. The idea for approach A1 is to rapidly find a potentially good map order using some initialization heuristic and introduce it into the initial PSO population. By doing this, the search should speed up while the quality of the final values for $\theta_{n}^{S B} \mathrm{~S}$ and $\theta_{n}^{H T} \mathrm{~s}$ are kept reasonably high. Experiments suggest that in the majority of cases, PSO finds a better map order than the one produced by the initialization heuristic. 


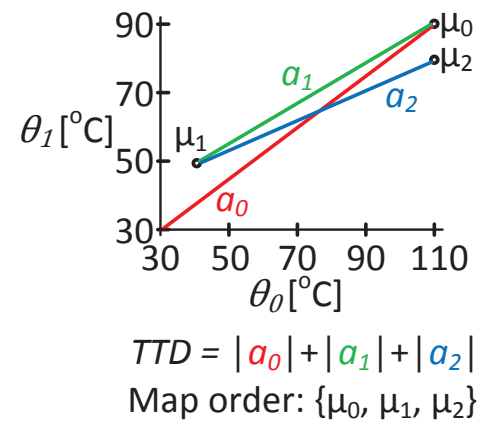

(a)

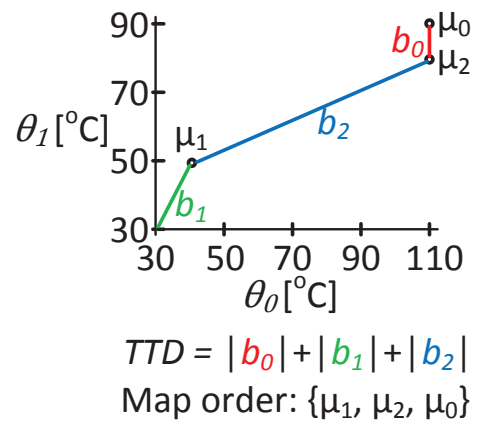

(b)

Figure 5.4.1 The total thermal distance (TTD)

(a) a bad map order. (b) a good map order.

It is, in fact, possible to find a potentially good map order without having to go through the time-consuming optimization of $\theta_{n}^{S B} \mathrm{~s}$ and $\theta_{n}^{H T} \mathrm{~s}$. Furthermore, it is possible to do it without the relatively time consuming scheduling procedures for the heating sequences. A temperature map could be considered as a point in an $N$-dimensional Euclidean space ( $N$ is the number of thermal nodes). The thermal distance between two maps is defined as the Euclidean distance between them (e.g., $b_{2}$ between maps $\mu_{1}$ and $\mu_{2}$ in Figure 5.4.1b). For a sequence of the maps, the total thermal distance (TTD) is defined as the sum of the thermal distances between successive maps. For example, TTD for Figure 5.4.1a is approximately 257, while for Figure 5.4.1b it is 108, which is much smaller. In general, a sequence of maps with smaller TTD is expected to have a shorter transition time compared with a sequence with larger TTD.

Note also that the time required to change the temperature differs from node to node depending on the node's location on the IC, the adjacent nodes' temperatures, the heating sequence powers, and so on. Moreover, depending on these factors, the rise time and the fall time for the temperature of a certain node are also different (e.g., in many cases heating up is faster than cooling down, with the same temperature gap). The TTD does not take these differences into account in favor of a simple but meaningful metric that is fast to evaluate. However, when the map order is optimized using PSO, all these once ignored factors are automatically taken into account. 
This problem is similar to finding the shortest Hamiltonian path in a complete graph whose vertices are temperature maps and the distance between two vertices is their Euclidean distance. Therefore, the initial heuristic based map order that is added to the PSO's initial population in approach A1 is called shortest Hamiltonian path. Due to the reasons discussed previously, this shortest path does not necessarily correspond to the optimal map order.

If A1 is allowed to run for a long time, it will produce very high quality solutions. However, for larger designs, this is unaffordable. We have therefore proposed the A2 approach, which consists of a short run of A1 followed by a post-PSO optimization of map orders. The motivation for this is that PSO optimization in A1 can rapidly identify possible solutions in the near optimal area of the search space but it then becomes very slow. Knowing the near optimal area, other optimization techniques can be deployed to rapidly improve the results. In the followings, the post-PSO optimization for the map orders is discussed.

In the general case, the post-PSO optimization could be excessively time consuming. A greedy heuristic is therefore used to rapidly find a near optimal solution. The greedy approach is characterized by its size, $S$. This size is the number of alternative partial solutions that are kept at each step (i.e., among the vertices with equal depth in the search tree). A greedy heuristic with size $S$ works as follows. Starting from the root vertex (initial temperature) in the search tree, $S$ vertices (i.e., $S$ temperature maps) that have the shortest partial transition times are selected. This corresponds to the first map in the final map order. Here the scheduling is performed to calculate the actual transition times.

Then again $S$ new vertices that have the shortest partial transition times are selected out of the set of vertices that succeed the previous best $S$ vertices. Two maps (in the final map order) are scheduled so far. This procedure repeats until all maps are scheduled. For $S$ equal to one, at each step the map that is the fastest to achieve is selected. A large $S$ slows down the search but it may provide better results. Our experiments showed that 10 is a good choice for $S$.

Albeit this general case which addresses large and time consuming ICs, for smaller ICs it is possible to find the optimal map order (i.e., exact solution) using an exact algorithm (e.g., branch and bound). Since a relatively good solution is already found by PSO in approach A1, we can skip many paths 
in the search tree that result in a larger transition time, without wasting time to fully schedule them. For example assuming that the map order in Figure $5.4 .1 \mathrm{~b}$ is already found by $\mathrm{A} 1$, there is no need to schedule $a_{2}$ (in Figure 5.4.1a) at all. Scheduling $a_{1}$ may also be aborted before completion since the overall transition time of this path in the search tree exceeds the overall transition time of the path corresponding to Figure $5.4 .1 \mathrm{~b}$ before it even gets to vertex $\mu_{1}$. Note that in this algorithm, the edges are actual transition times and not the Euclidean distances. Albeit significant acceleration achieved by utilizing the near optimal result from A1 approach, larger examples are excessively time consuming and therefore finding their optimal solution is not practical.

Although this section has focused on map ordering for the temperaturegradient based burn-in, the map ordering for the delay test is very similar and the same technique can be used. Moreover, there might be a map dependency graph (e.g., because of corresponding tests' dependencies) which dictates that certain maps must be applied in certain order. Although not discussed in this section, the proposed approach can accommodate such scenarios.

\subsubsection{Experimental Results}

Experimental setup is similar to section 5.2.6. All experiments are performed on a desktop computer with Intel ${ }^{\circledR}$ Xeon ${ }^{\circledR}$ W3520 processor and $8 \mathrm{~GB}$ of memory. Percentage change in CPU time for the A1 approach compared with the naïve approach is $-266 \%$ in average. Furthermore, the overall transition time achieved by A1 is $18 \%$ smaller than the overall transition time achieved by the naïve approach.

Optimal map orders are found for some of the small experimental ICs to be used for comparison purposes. It is not practical to find optimal map orders for all the experimental ICs because of the excessive search time that relatively large ICs require. The overall transition times achieved by A1 are around 23\% larger than the overall transition times offered by the optimal map orders. As mentioned before, this shows that the map orders found by A1 are close to optimal, but A2 can do better. In the following $\mathrm{A} 2$, that includes post-PSO optimization, is compared with A1 that terminates after the PSO optimization.

The greedy approach with a population size of one $(S=1)$ is used to find map orders for all of the experimental ICs. The results show $16 \%$ 


\section{Chapter 5}

improvement over the A1 results, but it is $13 \%$ worse than the optimal. Increasing the population size to ten $(S=10)$, further improves the results so that there is $21 \%$ improvement over the $\mathrm{A} 1$ and it is only $7 \%$ worse than the optimal. However, it almost doubles the search time. In short, A1 finds map orders that result in overall transition time around $23 \%$ worse than optimal. The post-PSO optimization in A2 improves the map orders by $21 \%$, which means that it is very close to the optimum.

\subsection{Conclusions}

Early-life failures and delay faults that are dependent on temperaturegradients introduce additional challenges to achieve efficient burn-in and delay-fault test. The negative effects of temperature gradients are more pronounced for 3D-SIC technology, since their magnitude is much larger. The challenge for burn-in is that some defects develop and cause early-life failures very rapidly when the IC is working with certain temperature maps that include large temperature gradients. These are difficult to enforce by traditional burn-in methods. The challenge for delay-fault test is that some defects can be detected only when a certain temperature map is enforced on the IC.

In order to effectively detect these defects, it is necessary to construct and maintain the specified temperature maps during burn-in and delay-fault test. The methods proposed in this thesis utilize the available test access mechanisms in order to do so. The specified temperature maps are constructed and maintained by selectively applying high-power stimuli to the IC. Therefore, there is no need for expensive equipment to heat up the chip externally. To our knowledge, this is the first technique to achieve temperature maps for burn-in and test without any external heating mechanism.

For burn-in, a steady state solution is introduced that is fast to generate the schedules, but the schedules are slow to achieve the specified temperatures. A schedule in this case consists of a single periodic schedule for each map. The steady state solution has been extended to the transient solution which is slow in generating the schedules, but constructs the maps faster. Finally, the transient-based heuristic is proposed to support a more precise temperature model, and offer a shorter overall transition time by generating schedules that rapidly bring the IC to the specified temperature conditions. The experiments indicate that this method outperforms the transient 
solution. Moreover, this method is $78 \%$ faster than the steady state solution in realizing the specified temperature maps.

For delay-fault test, a straightforward method is proposed that is based on two working modes, the temperature construction mode and the test mode. The temperature construction mode works similar to the transient-based method for burn-in and brings the IC to the specified temperature conditions. Then, the test mode applies the tests according to a given test schedule until the IC's temperatures exits the specified range, when the temperature construction mode is activated again. This continues until all tests are performed. Furthermore, another method (fast heuristic) has been developed to schedule the heating and cooling intervals mixed with the tests. Therefore, the test time offered by this method is reduced. The experiments indicate that the fast heuristic is $67 \%$ faster in performing the tests compared with the straightforward method.

The order of the temperature maps has a considerable effect on the overall burn-in and test time. Therefore, map orders need to be optimized, since they affect the optimal values for other decision variables. Experiments for map ordering show that the introduction of an initialization heuristic that adds an initial map order to the PSO's initial population speeds up the search time by $266 \%$ in average. Furthermore, the overall transition time improves by $18 \%$ in average for burn-in. The overall transition times are further improved by $21 \%$ through introduction of a post-PSO optimization stage that consists of a greedy approach. 


\subsection{Notations and Abbreviations}

\begin{tabular}{|c|c|}
\hline Notation & Description \\
\hline $\begin{array}{l}\boldsymbol{A} \\
a_{i, j}\end{array}$ & $\begin{array}{l}\text { Represents heat capacitances in the thermal model. } a_{i, j} \text { is the } \\
\text { matrix element at } i \text {-th row and } j \text {-th column. }\end{array}$ \\
\hline $\begin{array}{l}\boldsymbol{B} \\
b_{i, j}\end{array}$ & $\begin{array}{l}\text { Represents thermal conductance (related to heat transfer) in the } \\
\text { thermal model. } b_{i, j} \text { is the matrix element at } i \text {-th row and } j \text {-th } \\
\text { column. }\end{array}$ \\
\hline $\begin{array}{l}\boldsymbol{D} \\
d_{n}\end{array}$ & $\begin{array}{l}\text { Need for heating in a general case. } d_{n} \text { is } n \text {-th thermal-element's } \\
\text { need for heating. }\end{array}$ \\
\hline$D_{m}$ & Duty cycle for module $m$ in PWM method \\
\hline$e_{m}$ & Testing endurance for module $m$. \\
\hline$I$ & Identity matrix \\
\hline $\begin{array}{l}M \\
m_{i}\end{array}$ & $\begin{array}{l}\text { Number of modules }(m=0,1, \ldots, M-1) \text {. } \\
m_{i} \text { is the } i \text {-th module. }\end{array}$ \\
\hline$N$ & $\begin{array}{l}\text { Total number of thermal elements in the thermal model }(n= \\
0,1, \ldots, N-1)\end{array}$ \\
\hline $\begin{array}{l}\boldsymbol{P} \\
p_{m}\end{array}$ & Power value(s) in a general case. $p_{m}$ is power for module $m$. \\
\hline $\boldsymbol{P}^{B}$ & Power values in transient solution \\
\hline $\boldsymbol{P}^{H S}$ & Heating sequences' powers \\
\hline$p_{n, o}^{H S}$ & $\begin{array}{l}\text { Heating sequence power received by node } o \text { when heating is } \\
\text { intended for node } n \text {. }\end{array}$ \\
\hline $\boldsymbol{P}^{S S}$ & Steady state power values in transient solution \\
\hline$\overline{\boldsymbol{P}}$ & Stray power \\
\hline PSO & Particle Swarm Optimization [Poli07] \\
\hline$R$ & Number of parallel LP solvers in transient solution \\
\hline$r_{m}$ & Remaining tests' size \\
\hline$T_{m}^{H}$ & $\begin{array}{l}\text { Proper schedule period in PWM method, calculated solely for } \\
\text { heating interval of module } m\end{array}$ \\
\hline
\end{tabular}




\begin{tabular}{|c|c|}
\hline Notation & Description \\
\hline$T_{m}^{L}$ & $\begin{array}{l}\text { Proper schedule period in PWM method, calculated solely for } \\
\text { cooling interval of module } m\end{array}$ \\
\hline TAM & Test Access Mechanism \\
\hline TAT & Test Application Time \\
\hline$t t_{m}$ & Thermal tolerance for module $m$. \\
\hline TTD & Total Thermal Distance \\
\hline$W$ & $\begin{array}{l}\text { TAM width: number of modules that can be accessed at the same } \\
\text { time }\end{array}$ \\
\hline $\begin{array}{l}\alpha \\
\alpha(t)\end{array}$ & $\begin{array}{l}\text { Transfer matrix for initial temperatures considering a time interval } \\
\text { equalt to } t\end{array}$ \\
\hline $\begin{array}{l}\boldsymbol{\beta} \\
\boldsymbol{\beta}(t)\end{array}$ & $\begin{array}{l}\text { Transfer matrix for power values considering a time interval equalt } \\
\text { to } t\end{array}$ \\
\hline$\gamma_{r}$ & $\begin{array}{l}\text { Boolean variable indicating that the } r \text {-th LP solver has found a valid } \\
\text { solution }\end{array}$ \\
\hline$\Delta_{k}$ & Thermal distance for $k$-th active thermal element. \\
\hline$\varepsilon$ & Accpeptable error in the minimal transition time in trasient solution \\
\hline $\begin{array}{l}\boldsymbol{\Theta} \\
\theta_{m} \\
\theta_{n}\end{array}$ & $\begin{array}{l}\text { Temperatures vector in a general case. } \theta_{m} \text { is the temperature for } \\
\text { module } m . \theta_{n} \text { is the temperature for } n \text {-th thermal element. }\end{array}$ \\
\hline$\theta^{\text {ambient }}$ & Ambient temperature \\
\hline$\theta^{\text {over heating }}$ & Overheating temperature limit \\
\hline$\Theta^{0}$ & Initial temperatures \\
\hline $\boldsymbol{\Theta}^{t}$ & Final temperatures after $t$ seconds \\
\hline $\begin{array}{l}\boldsymbol{\theta}^{S B} \\
\theta_{n}^{S B}\end{array}$ & $\begin{array}{l}\text { Stop-boosting temperature limit in a general case. } \theta_{n}^{S B} \text { is stop } \\
\text { boosting limit for } n \text {-th thermal element. }\end{array}$ \\
\hline $\boldsymbol{\theta}^{S S}$ & Steady state temperatures \\
\hline $\begin{array}{l}\theta_{m}^{H} \\
\theta_{n}^{H}\end{array}$ & $\begin{array}{l}\theta_{m}^{H} \text { is high temperature limit for module } m . \theta_{n}^{H} \text { is high temperature } \\
\text { limit for } n \text {-th thermal node. }\end{array}$ \\
\hline
\end{tabular}




\section{Chapter 5}

\begin{tabular}{ll}
\hline Notation & Description \\
\hline$\theta_{m}^{L}$ & $\theta_{m}^{L}$ is low temperature limit for module $m . \theta_{n}^{L}$ is low temperature \\
$\theta_{n}^{L}$ & limit for $n$-th thermal node. \\
\hline $\boldsymbol{\theta}^{T T}$ & Testing-trigger temperature threshld in a general case. $\theta_{n}^{T T}$ is \\
$\theta_{n}^{T T}$ & testing trigger threshold for $n$-th thermal element. \\
\hline$\lambda$ & Lower bound for optimal transition time in transient solution. The \\
& upcoming temperature map cannot be achieved if transition time \\
& is smaller than $\lambda$. See $\sigma$. \\
\hline$\mu_{i}$ & $i$-th temperature map. \\
\hline
\end{tabular}




\section{Chapter 6 Integrated Temperature- Cycling Acceleration and Test}

Large and frequent temperature changes (i.e., temperature cycling) create fatigue and wearout in Integrated Circuits (IC), as pointed out earlier in section 3.8. Temperature-cycling affects ICs by causing various damages, including solder joint fatigue, fracture in bond wires, and die deformation [Jedec10]. In addition to these undesirable effects, 3D stacked ICs suffer from defects related to through silicon vias. TSV protrusion and void formation in TSV are two of such defects. These effects are worsened by temperature cycling. Furthermore, some other defects, including resistive opens and stress induced carrier mobility reduction, can also be worsened by temperature cycling [Kumar12, Okoro14, Zhang13].

This chapter presents a schedule-based technique that integrates temperature cycling acceleration with testing procedure. The cycling acceleration is achieved by mixing heating sequences and cooling intervals with test sequences in an efficient order. Furthermore, tests and heating sequences are reordered so that a rapid testing and acceleration process is achieved. The proposed technique is in contrast with the existing approaches that are based on temperature chambers and can be impractical for 3D-SICs due to their unaffordable costs and limitations.

\subsection{Preliminaries}

Temperature-cycling exacerbates a number of defect mechanisms, as pointed out before. Therefore, operating the dies under intensive temperature cycling can effectively accelerate such failures so that they can be detected by the subsequent test, before the 3D-SIC is shipped out. This procedure is called temperature-cycling acceleration [Jedec09, Mil04]. 
Note that even though both conventional burn-in test and temperaturecycling test are designed to detect early-life failures, temperature-cycling is different from the conventional burn-in. These two aim at accelerating different aging mechanisms. Cycling acceleration will not accelerate aging mechanisms identical to those that burn-in does and vice versa. To briefly explain this difference, let us focus only on two distinct aging mechanisms. During burn-in, the device is operated in a very hot environment with increased voltage to accelerate electromigration. This must continue for a relatively long time to allow for sufficient migration (detectable atomic built-up or depletion). On the contrary, simply operating the device at a single temperature does not create cycling-related material fatigue. It is the variation of the mechanical stress (as a result of varying temperature) that does it. The required amounts of burn-in and cycling are decided based on analytical, experimental, and empirical studies that are outside the scope of this thesis. In this thesis we solely focus on temperature-cycling and assume that the required amount of cycling is given by the user.

Let us have a closer look at protrusion of TSVs out of the die surface caused by temperature cycling. Right after TSV fabrication, there is normally no protrusion and the TSVs have about the same length as the die's thickness. However, after a few temperature-cycles an increase in the TSV length may be observed. The TSV length will continue to increase with the number of cycles [Kumar12, Zhang13]. After a certain amount of temperature cycling, the TSV length approaches a maximum level. Further temperature cycling will have almost no effect on the TSV length, afterwards. The TSV protrusion can be further exacerbated by the electrical current it carries [Kumar12, Zhang13]. Therefore, operating the IC during this procedure (letting the current to flow) speeds up the cycling acceleration.

The existing procedure for temperature-cycling acceleration is based on one or multiple temperature chambers [Jedec09]. Although this procedure is usually affordable for 2D ICs, it is likely to be too expensive for 3DSICs. Due to TSV-related defects, a larger number of dies manufactured to be a part of a 3D-SIC may require cycling acceleration compared with 2D ICs. The shortcomings of the traditional approach include costs for running the temperature chambers as well as the time and equipment required for handling the dies/stacks between test equipment and chambers. Besides, chambers are slow, meaning that only very low frequency cycling is possible. 
Moreover, the 3D-SIC manufacturing process includes multiple bonding stages. Corresponding to these bonding stages, pre-, mid-, or post-bond tests are introduced in order to avoid: (1) wasting a good die bonded to a bad die or stack, (2) wasting bonding effort for bonding bad dies or stacks, and (3) wasting packaging effort spent on a bad stack. Based on the cost breakdown, temperature-cycling acceleration could be beneficial at one or multiple test stages. In order to avoid costs associated with the traditional techniques, in current practice, some or even all of the temperature-cycling acceleration operations are avoided. Therefore, the temperature-cycling related early-life failure rates in the final products will be unnecessarily high. Integrating the temperature-cycling acceleration with the tests that are performed at different stages and eliminating the need for temperature chambers will reduce the overall manufacturing costs.

As previously mentioned, advanced SoCs, especially those manufactured as a 3D-SIC experience excessively large test power densities during test. High power densities lead to excessively high temperatures, in particular for the middle dies in a 3D stack. This otherwise undesirable thermal effect is, however, utilized here to generate large amounts of temperaturecycling. Temperature-cycling acceleration is achieved by frequent switching between high power tests that heat up the IC and pauses that allow for cooling.

A deliberate pause for cooling is called a cooling interval. A cooling interval is the time interval that no stimuli are applied to a core and, therefore, the core's temperature decreases, as already discussed in earlier chapters. Some cooling intervals are usually present in the original test schedule for thermal-safety reasons, as discussed in chapter 4 . More intensive temperature-cycling acceleration can be achieved by introducing additional cooling intervals and stronger heating sequences into the process. A stronger heating sequence consists of stimuli that generate larger switching activities in a core and, therefore, increases the core's temperature faster than usual (as discussed in chapter 4 and chapter 5). The mixture of cooling intervals and heating sequences can generate the required temperature-cycling acceleration effect.

A test sequence's bit streams define the circuit-under-test's power dissipation in combination with the previously applied test sequence (circuit's state) as well as the core's power-related properties. Consequently, the power dissipation generated by a series of tests depends 
on the order in which they are applied [Chakravarty94]. This phenomenon is employed in this thesis in order to produce extreme power values for tests as well as heating sequences and, consequently, achieve a high speed temperature-cycling process.

The existing methods for managing ICs' temperatures (in relation with the testing processes) focus on two issues:

1. Keeping the temperatures under a global upper temperature limit to prevent overheating (e.g., section 4.1-7) or

2. To respect upper and lower bounds for cores in order to target temperature-dependent defects (e.g., section 4.8) or gradientdependent defects (chapter 5).

In all the above cases, the cores' temperatures are considered independent of their cycling effects. Integrating temperature cycling acceleration with the test procedure was previously studied in [Aghaee15a]. This chapter develops an integrated temperature cycling technique based on this study. Moreover, an efficient technique to order the tests and heating sequences to achieve a high-speed temperature-cycling process is proposed.

\subsubsection{Circuit under Test and Test Access Mechanism}

It is assumed that there are $M$ modules (cores) in the 3D-SIC under test. These modules are located on different levels of stacked dies. The modules that are on different layers are connected using TSVs. Tests for each module can be started and stopped independent of other modules. The modules could be cores with core wrappers in a core-based design. The extension of this scenario to 3D-SIC is proposed as the IEEE P1838 standard [Ieee14a]. Test stimuli are, therefore, transferred through a test access mechanism to the relevant module. It is assumed that the TAM only affords $W$ (a positive integer number) modules to be tested at the same time. Other modules, therefore, have to queue up and wait for TAM access.

\subsubsection{Thermal Model}

In order to obtain the temperature values from power values, a thermal model that describes the thermal behavior of the IC must be used. The temperature equation (introduced in section 2.6, equation 2.6.1) is repeated here for convenience:

$$
\boldsymbol{A} \times \frac{d}{d t} \boldsymbol{\Theta}+\boldsymbol{B} \times \boldsymbol{\Theta}=\boldsymbol{P}
$$


All the thermal characteristics of the IC are captured in two matrices ${ }^{1} \boldsymbol{A}$ and $\boldsymbol{B}$, obtained in a manner similar to [Coskun09, Huang06]. $\boldsymbol{\Theta}$ is the temperature vector and $\boldsymbol{P}$ is the power. $\boldsymbol{\Theta}$ and $\boldsymbol{P}$ consist of $\theta_{m} \mathrm{~s}$ and $P_{m} \mathrm{~s}$, respectively, put together in a vector format. Index $m$ indicates the relevant module. There are a total of $M$ modules $(m=0,1, \ldots, M-1)$. As discussed in section 4.6, equation 6.1.1 can be solved for the time-domain assuming that the power values are constant during a period of time equal to $\tau$. The result from equation 4.6.5 is repeated here for convenience:

$$
\boldsymbol{\theta}^{\tau}=\boldsymbol{\alpha} \times \boldsymbol{\theta}^{0}+\boldsymbol{\beta} \times \boldsymbol{P}
$$

The initial temperature is expressed by $\boldsymbol{\theta}^{0}$ and the temperature after a period of $\tau$ seconds (note that a fraction of a second is used in practice) is represented by $\boldsymbol{\theta}^{\tau}$. Matrices $\boldsymbol{\alpha}$ and $\boldsymbol{\beta}$ are copied below from equations 4.6.3-4:

$$
\begin{aligned}
& \boldsymbol{\alpha}=\exp \left(-\boldsymbol{A}^{-1} \times \boldsymbol{B} \times \tau\right) \\
& \boldsymbol{\beta}=(\boldsymbol{I}-\boldsymbol{\alpha}) \times \boldsymbol{B}^{-1}
\end{aligned}
$$

The identity matrix is denoted by $\boldsymbol{I}$. The above equations are explained in the following case study, assuming that there is only one module $(M=1)$ with its heat capacitance denoted by $C$ (analogous to $\boldsymbol{A}$ ). The heat resistance between the module and the ambient is equal to $R$ (analogous to $\left.\boldsymbol{B}^{-1}\right)$. In this case, equation 6.1 .2 can be re-written as:

$$
\theta^{\tau}=\theta^{0} \cdot \exp \left(-\frac{\tau}{R \cdot C}\right)+P \cdot R \cdot\left(1-\exp \left(-\frac{\tau}{R \cdot C}\right)\right)
$$

Since there is only one module, the vectors and matrices are reduced to scalar values. A larger initial temperature $\left(\theta^{0}\right)$, power $(P)$, or resistance $(R)$ results in higher final temperature $\left(\theta^{\tau}\right)$, if other factors are kept unchanged. A larger period $(\tau)$ means that the contribution of the initial temperature is smaller while the effect of power on the final temperature is larger. In the vector form, increasing the period translates into a decreased $\boldsymbol{\alpha}$ and an increased $\boldsymbol{\beta}$. A large time-constant $(R \cdot C)$ means that the initial temperature takes longer to lose its effect while power takes longer to noticeably affect the final temperature. In the vector form, increasing the time-constant translates into an increased $\boldsymbol{\alpha}$ and a decreased $\boldsymbol{\beta}$.

\footnotetext{
${ }^{1} \mathrm{~A}$ list of notations and abbreviations is provided in section 6.8 .
} 


\subsubsection{Temperature Cycling Model}

The effect of temperature cycling can be described based on the Amount of Temperature Cycling induced fatigue (denoted by $\mathrm{ATC}_{m}$ for module $m$ ). Based on the Arrhenius-Coffin-Manson model [Held97, Jedec10], ATC is estimated as:

$$
A T C_{m} \cong \frac{N_{m}}{k_{0}} \times\left(\frac{\Delta \theta_{m}-\theta^{\epsilon}}{k_{1}}\right)^{\gamma} \times \exp \left(\frac{\overline{\theta_{m}}}{k_{2}}\right)
$$

Considering module $m, \mathcal{N}_{m}$ is the number of temperature cycles and $\Delta \theta_{m}$ is the amplitude of temperature changes during cycling. In the above equation, a regular cycling pattern is assumed. It means that the temperature monotonically increases from an arbitrary temperature, $\theta_{m}^{a}$, to $\theta_{m}^{a}+\Delta \theta_{m}$ and then monotonically decreases back to $\theta_{m}^{a}$.

Usually, when the actual temperature curve is only slightly different from a regular pattern, the average amplitude is used for $\Delta \theta_{m} . \Delta \theta_{m}$ must be larger than $\theta^{\epsilon}$ (a very small threshold value) in order to be considered in the temperature cycling calculations. However, it is not unusual to completely ignore $\theta^{\epsilon}$ since the typical temperature changes are much larger than $\theta^{\epsilon}$.

The effect of the average temperature is captured in the exponential term. The average temperature is expressed by $\overline{\theta_{m}} \cdot \theta^{\epsilon}, k_{0}, k_{1}, k_{2}$, and $\gamma$ are constants that are obtained analytically or empirically by reliability analysts. A comprehensive explanation and details of equation $6.1 .5 \mathrm{can}$ be found in [Jedec10, Held97]. As equation 6.1.5 suggests, a large number of cycles, $\mathcal{N}_{m}$, or a large temperature swing, $\Delta \theta_{m}$, will result in a large cycling effect.

\subsection{Motivational Examples}

\subsubsection{ATC Rate for a Simple Scenario}

As an example, consider an IC with two modules $(M=2)$. Assume that the TAM can only support one module to be tested at a time $(W=1)$. Assume that $\theta^{\text {overheating }}=150^{\circ} \mathrm{C}$ and $\theta^{\text {ambient }}=30^{\circ} \mathrm{C}$. The required amounts of temperature cycling are $A T C_{0}^{R}$ and $A T C_{1}^{R}$ for modules $m_{0}$ and $m_{1}$, respectively. In this chapter, tests that target cycling-dependent defects are called cycling tests and the other tests are called normal tests. Cycling 
tests can only be applied after the required amount of temperature cycling, $A T C_{m}^{R}$, is achieved.

A three-phase approach is introduced here: In phase 1, normal tests are scheduled. A thermal aware scheduling of tests based on the proposed approach in $[\mathrm{He} 08 \mathrm{a}]$ is used. The corresponding temperature curves are shown in Figure 6.2.1 (green ${ }^{2}$ for $m_{0}$ and blue for $m_{1}$ ). The normal tests for module $m$ end at $t_{m}^{0}$. Phase 1 starts at time 0 and end at $t^{0}$ that is defined as $\max _{m}\left\{t_{m}^{0}\right\}$.

Phase 2 starts by evaluating the ATC generated in phase 1 . This value is less than the required $A T C_{m}^{R}$ in this example. Therefore, phase 2 will generate additional temperature cycling. This is done by applying the heating sequences and cooling intervals. Corresponding temperature cycles can be seen in Figure 6.2.1 from $t^{0}$ to $\hat{t}$. Time-point $\hat{t}_{m}$ marks the point when the required $A T C_{m}^{R}$ is achieved for module $m$. Phase 2 ends when all required ATCs for all modules are met. This point is marked with $\hat{t}$ that is defined as $\max _{m}\left\{\hat{t}_{m}\right\}$. After this, phase 3 starts by applying the cycling tests. Phase 3 ends when all the cycling tests are complete. This point is marked with $T A T$.

Always, a small TAT is desirable. Test application time from 0 to $t^{0}$ and from $\hat{t}$ to $T A T$ is already minimized by the given third-party test scheduling algorithm. The only time reduction opportunity is to speed up phase 2 . This means that a large ATC should be achieved in a short time. Therefore, $A T C_{m}(t) / t$ should be maximized. Here we assume a uniform periodic temperature profile that means all cycles have the same amplitude.

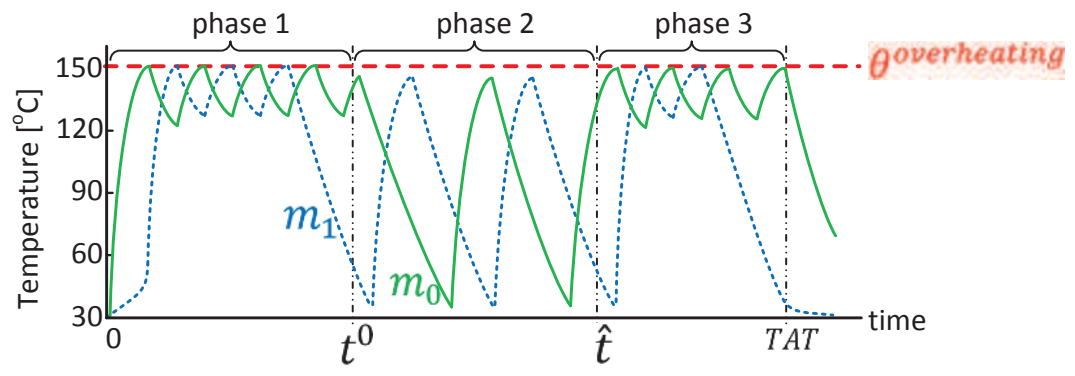

Figure 6.2.1 Temperature curves for the three-phase approach (Curves are illustrative.)

${ }^{2}$ Figure 6.2 .1 is printed in grayscale in copies printed by LiU-Tryck. 
Moreover, for this motivational example we assume that in equation 6.1.5: $k_{0}=1, k_{1}=1, k_{2} \gg \overline{\theta_{m}}$, and $\theta^{\epsilon} \ll \Delta \theta_{m}$.

Since it is assumed that $k_{2} \gg \overline{\theta_{m}}$, the exponential term can be ignored for the moment. Furthermore, since it is assumed that $\theta^{\epsilon} \ll \Delta \theta_{m}, \theta^{\epsilon}$ could also be ignored. The ATC rate (denoted by $\rho_{m}$ for module $m$ ) can, therefore, be defined as:

$$
\rho_{m}=\frac{A T C_{m}(t)}{t}=\frac{\mathcal{N}_{m}(t)}{t} \times\left(\Delta \theta_{m}\right)^{\gamma}
$$

The frequency of temperature changes (i.e., the number of cycles per time unit) depends on the physical properties of the system and the amplitude of temperature changes, $\Delta \theta_{m}$. It is possible to achieve a high frequency (i.e., a large $\frac{\mathcal{N}_{m}(t)}{t}$ ) if $\Delta \theta_{m}$ is small. A large amplitude on the other hand, may increase the ATC, only if it dominates the resulted reduction in the frequency.

\subsubsection{Optimal Cycling in a Simplified Scenario}

In order to clarify the tradeoff between the frequency and the amplitude of the temperature cycling, the physical properties of the system should be captured in the ATC rate equation (equation 6.2.1). In the following this is done for a simple IC with only one module. The thermal model for such a case was discussed in section 6.1.2, equation 6.1.4. Remember that $C$ is the heat capacitance and $R$ is the thermal resistance between the module and the ambient. Assume that the heating sequence generates a power equal to $P$ and the power during a cooling interval is zero. Assume that the temperature varies between $\mu-\sigma$ and $\mu+\sigma$. Both $\mu$ and $\sigma$ are positive real numbers.

The period of a temperature cycle is denoted by $T$. This period consists of a rise time denoted by $T_{r}$ plus a fall time denoted by $T_{f}$. $T_{r}$ is the time the temperature takes to increase from $\mu-\sigma$ to $\mu+\sigma . T_{f}$ is the time taken to decrease from $\mu+\sigma$ to $\mu-\sigma$. These values are calculated as follows. First, the system's differential equation is solved in the time domain similar to equation 6.1.4 for a period of $t$ (i.e., $\tau=t$ ):

$$
\theta^{t}=\theta^{0} \cdot \exp \left(-\frac{t}{R \cdot C}\right)+P \cdot R \cdot\left(1-\exp \left(-\frac{t}{R \cdot C}\right)\right)
$$

Let us denote $R \cdot C$ by $R C$ and $P \cdot R$ by $P R$. For the heating situation: 


$$
(\mu+\sigma)=(\mu-\sigma) \exp \left(-\frac{T_{r}}{R C}\right)+P R\left(1-\exp \left(-\frac{T_{r}}{R C}\right)\right)
$$

Then

$$
T_{r}=R C \times \ln \left(\frac{\mu-\sigma-P R}{\mu+\sigma-P R}\right)
$$

Similarly for cooling $(P=0), T_{f}$ can be calculated:

$$
T_{f}=R C \times \ln \left(\frac{\mu+\sigma}{\mu-\sigma}\right)
$$

The period, $T$, is calculated as follows:

$$
T=T_{r}+T_{f}=R C \times \ln \left(\frac{(\mu-\sigma-P R)(\mu+\sigma)}{(\mu+\sigma-P R)(\mu-\sigma)}\right)
$$

Now, the ATC rate (equation 6.2.1) could be re-written incorporating the physical properties of the system:

$$
\rho_{m}=\frac{(2 \sigma)^{\gamma}}{R C \times \ln \left(\frac{(\mu-P R)(\mu+\sigma)}{(\mu+\sigma-P R)(\mu-\sigma)}\right)}=\frac{(2 \sigma)^{\gamma}}{R C \times \ln (\xi)}
$$

Let us first focus on the optimal value for $\mu$, assuming that $\sigma$ is constant. In this case optimality happens when the denominator in equation 6.2.6 is minimized. Considering a realistic situation, this is equivalent to finding the minimum for

$$
\xi=\frac{(\mu-\sigma-P R)(\mu+\sigma)}{(\mu+\sigma-P R)(\mu-\sigma)}
$$

Following a closed-form approach:

$$
\frac{d}{d \mu} \xi=0 \rightarrow \frac{(2 \mu-P R)((\mu+\sigma-P R)(\mu-\sigma)-(\mu-\sigma-P R)(\mu+\sigma))}{((\mu+\sigma-P R)(\mu-\sigma))^{2}}=0
$$

The valid solution is $\mu=P R / 2$. Here for the sake of simplicity, the ambient temperature was not included in the equations. Since the temperature model is a linear time-invariant (LTI) system as discussed in section 4.6 the ambient temperature can be added later on. Assume that power and resistance values are so that $P R=120^{\circ} \mathrm{C}$. This means that considering the ambient temperature $\left(30^{\circ} \mathrm{C}\right)$, the IC's temperature will increase to $150^{\circ} \mathrm{C}$ if no control is applied. Thus, the optimal value for $\mu$ is $\mu_{\text {Optimal }}=\frac{120^{\circ} \mathrm{C}}{2}+30^{\circ} \mathrm{C}=90^{\circ} \mathrm{C}$. 
The resulted equations for finding the optimal value for $\sigma$ do not have a simple closed form. Therefore, a numerical method is employed. The ATC rate $\rho$ versus $\sigma$ for $\mu=90^{\circ} \mathrm{C}$ is plotted in Figure 6.2.2. If $\gamma=4$ and $R C=$ $50 \mu \mathrm{s}$, then the ATC rate is maximal at $\sigma_{\text {Optimal }}=55.6^{\circ} \mathrm{C}$. For values of $\sigma$ less than $\sigma_{\text {Optimal }}$ the ATC rate increases by increase in $\sigma$. This is due to the increase in amplitude, $\left(\Delta \theta_{m}\right)^{\gamma}$, dominating the decrease in frequency, $\mathcal{N}_{m}(t) / t$, in equation 6.2.1. For larger $\sigma$ values the ATC rate decreases by increase in $\sigma$. This is due to the increase in amplitude, $\left(\Delta \theta_{m}\right)^{\gamma}$, being dominated by the decrease in frequency, $\mathcal{N}_{m}(t) / t$. In other words, a very large temperature cycle takes too much time to complete.

If the assumption that $k_{2} \gg \overline{\theta_{m}}$ does not hold, the temperature cycling rate equation, equation 6.2.6, will be as follows:

$$
\rho_{m}=\frac{(2 \sigma)^{\gamma}}{R C \times \ln \left(\frac{(\mu-\sigma-P R)(\mu+\sigma)}{(\mu+\sigma-P R)(\mu-\sigma)}\right)} \times e^{\mu / K_{2}}=\frac{(2 \sigma)^{\gamma}}{R C \times \ln (\xi)} \times e^{\mu / K_{2}}
$$

The inclusion of the exponential (Arrhenius) term results in a larger (or equal) optimal $\mu_{\text {Optimal }}$ value. Since both the exponential term and equation 6.2.6 are increasing when $\mu$ is smaller than $P R / 2$, the optimal value cannot happen for a $\mu$ smaller than $P R / 2$. After this point, the value of equation 6.2.6 decreases while the exponential term is increasing. The optimal $\mu$ can be in this region $(\mu \geq P R / 2)$. Besides, the introduction of the exponential term leads to dependency of the optimal $\mu$ on the value of $\sigma$.

In the general case (without assumptions made solely for the motivational examples), the optimal value for $\mu$ could be very different compared with the $\mu_{\text {Optimal }}$ obtained here. Moreover, the assumptions made for obtaining equation 6.2.1 will not be valid and therefore the situation will be more complicated than discussed in the above paragraph. In such situations a numerical approach is best suited to find the optimal values for $\mu$ and $\sigma$.

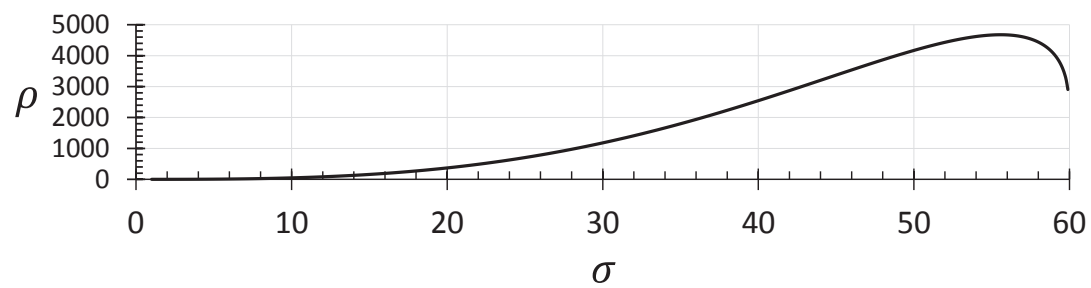

Figure 6.2.2 ATC rate, $\rho$, versus $\sigma$ for three-phase approach 
Moreover, in the general case, there are multiple modules competing for access to TAM and their interference makes the problem even more complicated, so complex that a heuristic is the only practical technique to deal with the problem.

\subsubsection{Effect of the Test Application Order}

In general, the circuit under test's consumed power depends on the order in which the tests are performed. Let us consider the scan chain itself. Different orders of the tests will result in different transition counts and thus different power values.

Consider a 4-bit scan chain as shown in Figure 6.2.3. Assume that 0101, 1111, and 1010 are the test stimuli. The order 1010-1111-0101, as shown in Figure 6.2.3a, results in 12 transitions in the scan chain during shift-in. Another test order, 1111-1010-0101, as shown in Figure 6.2.3b, results in 22 transitions and thus higher power dissipation. Assuming that the temperature of the core should be reduced, arranging the tests in their low power order may avoid an additional cooling interval. Alternatively, if the core is in its heating interval of the cycling process, the high power arrangement may replace an unnecessary heating sequence application. This will ensure that TAM is not unnecessarily occupied by dummy heating sequences. Both situations help to shorten the test application time.

\subsection{Problem Formulation}

As discussed before, along with pre-, mid-, or, post-bond tests, temperature-cycling acceleration might be beneficial. In this case, there will be tests that target cycling-dependent defects (i.e. cycling tests) in addition to other tests (i.e., normal tests). Normal tests are scheduled along with heating and cooling intervals in order to generate the required amount of temperature cycling. The cycling tests can be performed afterward.
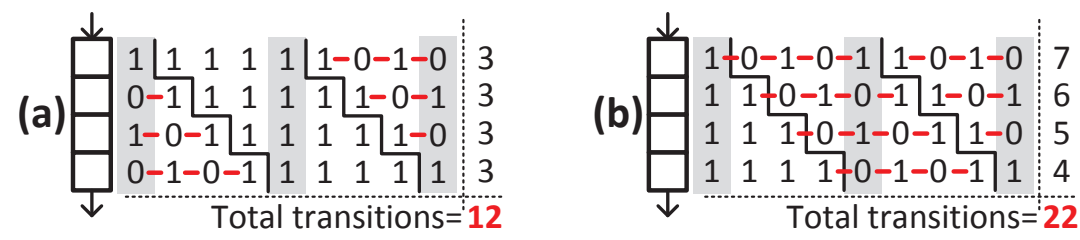

Figure 6.2.3 Test orders

(a) A low power order. (b) A high power order. 
The amount of temperature cycling can be easily calculated using equation 6.1.5 if the temperature swings in a uniform periodic manner similar to Figure 6.3.1a. In Figure 6.3.1a five cycles with amplitudes equal to $\Delta \theta$ can be identified. In the general case, for example when the IC is under test, the temperature fluctuations are irregular, as shown in Figure 6.3.1b. In this case, identifying cycles and their amplitudes is not straightforward. For such irregular patterns, the number and amplitudes of the cycles are calculated using the widely used Rainflow-counting algorithm [Matsuishi68].

As mentioned previously, the required amount of temperature cycling is denoted by $A T C_{m}^{R}$. The current amount of temperature cycling generated by normal tests or heating sequences (e.g., phase 1 and phase 2 in Figure 6.2.1), up to a given time, $t$, is denoted by $A T C_{m}(t)$. For a certain test schedule, the temperature curves are obtained using temperature simulations. Then a fast version of the Rainflow-counting algorithm, introduced in [Musallam12], calculates $A T C_{m}(t)$.

Assuming that for $t<\hat{t}_{m}, A T C_{m}(t)<A T C_{m}^{R}$, only normal tests can be performed before time $\hat{t}_{m}$. The cycling tests can only be performed after the required amount of cycling $\left(A T C_{m}^{R}\right)$ has been applied. Therefore, after time $\hat{t}_{m}$, cycling tests can be performed too. The test application time, $T A T_{m}$, marks the point that testing module $m$ is complete. $T A T_{m}$ consists of the time spent before and after time $\hat{t}_{m}$. The goal is to generate a schedule with a minimal overall TAT. The overall test application time is defined as $\max _{m}\left\{T A T_{m}\right\}$.

As previously discussed, the power dissipation during a test depends on the previous test, among other factors. Assuming that test $s_{m, s}$ for module $m$ immediately follows test $s_{m, u}$, the dynamic power is expressed by $p_{m, u-s}^{d}$. The overall power dissipation (in the circuit under test), denoted by $p_{m, u-s}$, consists of the dynamic power, $p_{m, u-s}^{d}$, plus the stray power,

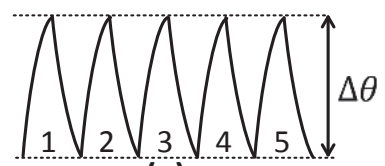

(a)

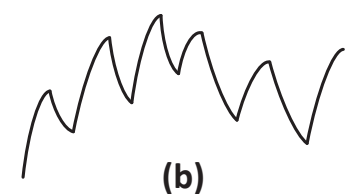

(b)

Figure 6.3.1 Temperature patterns

(a) Uniform periodic. (b) Irregular. 
denoted by $\widehat{p_{m}}\left(p_{m, u-s}=p_{m, u-s}^{d}+\widehat{p_{m}}\right)$. The dynamic power is caused by the circuit under tests' switching activities. As introduced in section 5.2.2, the stray power is defined, in this thesis, as the sum of all those power values whose dissipations cannot be independently controlled with existing test controls. This includes the leakage power as well as the clock networks' power. Stray power's exact value depends on the module's current temperature since the leakage power depends on the temperature. In this chapter, the stray power (including temperature dependent leakage) is taken into account.

It is assumed that module $m$ has $N_{m}$ tests including both normal and cycling tests. Relevant test properties can be captured in a test graph. Consider an IC that consists of two modules $(M=2)$. Assume that module $m_{0}$ has two tests $\left(N_{0}=2\right)$ as shown in Figure 6.3.2a. Module $m_{1}$ has three tests $\left(N_{1}=3\right)$ as shown in Figure 6.3.2b. Assume that one of the tests for module $m_{0}$ is a normal test (the node is marked with $\mathrm{N}$ ) and the other is a cycling test (marked with $\mathrm{C}$ ). A node that corresponds to a heating sequence (marked with $\mathrm{H}$ ) is also included in the test graph. Tests and the heating sequence for module $m_{1}$ are marked in a similar manner. Total test powers are shown on the edges in Figure 6.3.2. Usually, in the general case, there are a number of normal and cycling tests in addition to a number of heating sequences.

At each time point, during the test, there could be some tests that cannot be performed. This is due to a number of reasons, including the limited

(a)

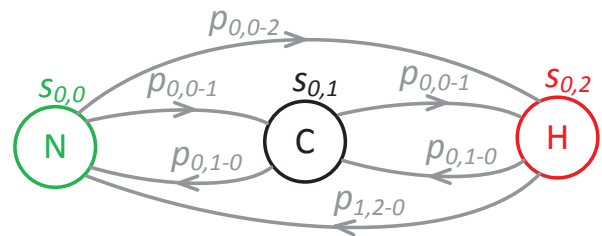

(b)

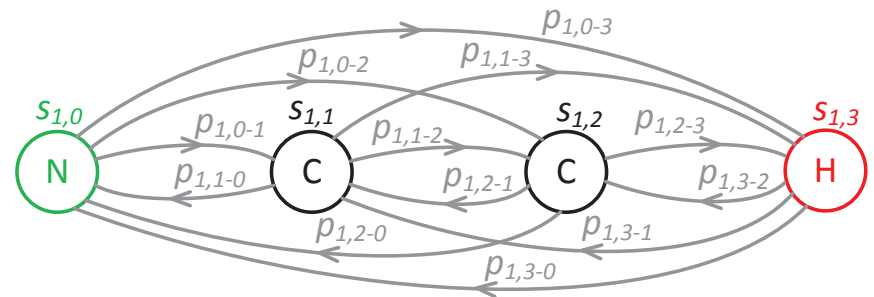

Figure 6.3.2 Test graphs: (a) module $\boldsymbol{m}_{\mathbf{0}}$ (b) module $\boldsymbol{m}_{\mathbf{1}}$.

Test graphs consist of normal $(\mathrm{N})$, cycling $(\mathrm{C})$, and heating $(\mathrm{H})$ nodes. 
capacity of the TAM as well as the cycling tests that cannot be performed before the required ATC is applied. A validity checker is used to make sure that the scheduling algorithm takes these limitations into account. The validity checker updates the set of Valid Tests (VaT) if a new test can be performed in parallel with the tests that are already selected for the current time point. It also makes sure that any test that cannot be applied in parallel with the currently selected tests does not remain in VaT. This is based on the knowledge of previously applied tests as well as the partial set of tests selected to be applied next.

Moreover, the current amount of the ATC is also taken into account. For example, assume that in Figure 6.3.2 normal tests $\left(s_{0,0}\right.$ and $\left.s_{1,0}\right)$ have been performed previously. Assume that $s_{0,1}$ is already selected to be applied next and the required ATC for $m_{1}$ is already achieved. In this case VaT is $\left\{s_{1,1} s_{1,2} s_{1,3}\right\}$. Meaning that $s_{1,1}, s_{1,2}$, or $s_{1,3}$ can be applied in parallel with $s_{0,1}$ without violating TAM limit or ATC requirement. Although using $s_{1,3}$ (i.e., the heating sequence) does not make sense since the required ATC is already achieved, it would be a valid choice from the VaT's point of view. Note that the heating sequences can be applied repeatedly, as needed, while repeating the tests is usually unnecessary.

The goal is to schedule the tests so that all the cycling tests are performed after the required amount of ATC is achieved and the overall test application time (including the cycling process) is minimized. This is achieved by scheduling and reordering the tests and the heating sequences. High power test stimuli and heating sequences can increase the modules' temperatures. A module may become so hot that unrealistic failures show up and even the device gets damaged. In order to avoid these undesirable overheating situations, the modules' temperatures must be kept below the overheating temperature ( $\left.\theta^{\text {overheating }}\right)$ at any time. The overheating temperature is equal to the temperature limit minus a safety margin to ensure thermal safety. The power dissipation during a pause is equal to the stray power, $\widehat{p_{m}}$ (which includes leakage).

The problem can be formally stated as follows. The inputs to the suggested technique include the IC's thermal model, the IC's electrical model (e.g., specification of the TAM and power-related specifications), the test graph (i.e., the cycling tests, normal tests, and the switching activities of the tests and heating sequences), the ambient temperature ( $\left.\theta^{\text {ambient }}\right)$, and the required amount of temperature cycling, $A T C_{m}^{R}$. The objective is to 
minimize the test application time. The output is the corresponding schedule that guides the application of the tests and heating sequences in proper order so that all the tests are performed rapidly and correctly.

The generated schedule will imply, for each of the modules, a certain ordering of the test graph's node. The ordering can be represented by a directed path in each of the original test graphs (e.g., graphs in Figure 6.3.2). This directed path must visit each test node at least once and may visit heating nodes as many times as needed. Applying a test or a heating sequence is equivalent to visiting the corresponding test or the heating node.

The test ordering and scheduling can also be viewed as converting the original test graph into a final path-graph. A path-graph is defined as a graph with only one directed path that connects all the nodes. There is no other edge in a path-graph except those on this unique path. The final pathgraph must include all of the test nodes, while the heating nodes are included as needed. The complete test scheduler that includes the ordering algorithm decides at which point to insert a node taken from the original test graph into the final path-graph.

\subsection{Three-Phase Approach}

The basics of the three-phase approach are briefly explained in section 6.2.1. Section 6.2.2 presented a technique to find the best temperature interval $(\mu-\sigma$ to $\mu+\sigma)$ for a simplified scenario. As discussed before, if the coefficient $k_{2}$ (in equation 6.1.5) is much larger than the average temperature $\left(k_{2} \gg \overline{\theta_{m}}\right)$ and the high temperature level $(\mu+\sigma)$ is smaller than the overheating temperature, $\theta^{\text {overheating }}$, everything in section 6.2 would be fine.

However, often these assumptions are not valid, for example the overheating temperature may be relatively low compared with $\mu+\sigma$. For the example in section $6.2 .2, \mu+\sigma$ is equal to $145.6^{\circ} \mathrm{C}$ while the overheating temperature might be $120^{\circ} \mathrm{C}$. There are some other complications, as well. In practice there are a number of modules, instead of one, and their temperatures depend on each other due to heat transfer.

Moreover, the power values fluctuate with time. Besides, power values include the stray powers that depend on the temperature due to the temperature dependent leakage currents. Additionally, the modules may 
not be able to receive their heating sequences at desired times, due to the TAM limitation. New approaches capable of taking all these situations into account are, therefore, proposed in this section.

As discussed in section 6.2.1, in phase 1 and 3 the tests are scheduled using a thermally safe third-party algorithm. It is assumed that these algorithms perform optimization to reduce the test application time. Our focus will therefore be on phase 2 where new algorithms can be designed to minimize the test application time. This was demonstrated using a small example in section 6.2.2. Assume that in phase 2 the temperature of module $m$ is intended to swing between a low temperature level $\theta_{m}^{L}$ and a high temperature level $\theta_{m}^{H}\left(\theta_{m}^{L}<\theta_{m}^{H}\right)$. In comparison with the example in section 6.2.2, $\theta_{m}^{L}$ and $\theta_{m}^{H}$ have roles similar to that of $\mu-\sigma$ and $\mu+\sigma$, respectively.

The heating sequences are assumed to be powerful enough to raise the module's temperature to $\theta_{m}^{H}$. The high temperature level should always be lower than the overheating temperature $\left(\theta_{m}^{H}<\theta^{\text {overheating }}\right)$ to avoid any kind of damage. Since all the normal tests and all the cycling tests are to be separately scheduled using third party algorithms and then performed in two isolated phases (phase 1 and 3), there is no need to represent them in the test graph. Consequently the test graph reduces to only include the heating nodes (nodes marked with $\mathrm{H}$ in Figure 6.3.2). This simplifies the problem of finding proper paths in these reduced graphs. For each module, a greedy approach is used here and the heating node that offers the highest heating power is selected to follow the current node.

An on-the-fly approach is used to schedule the heating sequences for phase 2 based on the simulated temperatures. The temperatures that are obtained by simulation are then compared with $\theta_{m}^{L}$ and $\theta_{m}^{H}$ in order to generate the schedule. High power heating nodes are used to rapidly increase the temperature. Immediately after the temperature reaches its peak at $\theta_{m}^{H}$, a cooling interval is introduced to reduce the temperature back to $\theta_{m}^{L}$. Then, for the sake of a fast cycling, the heating sequence must be immediately applied again. However, the TAM might not be available at this moment. Consequently, the temperature may fall below $\theta_{m}^{L}$ from time to time. Heating sequences for different modules will compete for access to TAM. The priority is decided based on the following equation: 


$$
\pi_{m}=\left(\theta_{m}^{L}-\theta_{m}\right) \times \frac{A T C_{m}^{R}}{\epsilon+A T C_{m}}
$$

Both $\theta_{m}$ and $A T C_{m}$ depend on time and are shortened forms of $\theta_{m}(t)$ and $A T C_{m}(t)$, respectively, at time $t$. The priority is higher if the module's current temperature is much below $\theta_{m}^{L}$. Note that the priorities are calculated only for modules that need heating, therefore $\theta_{m}<\theta_{m}^{L}$. The reason for the inclusion of this difference term (i.e., $\theta_{m}^{L}-\theta_{m}$ ) in the priority assessment is that if a module gets really cold, it takes too much time to warm it up again. Therefore, it is a good idea to give a higher priority to the colder modules.

A module that has a large amount of temperature cycling left to fill has also a higher priority. This is indicated by $\frac{A T C_{m}^{R}}{\epsilon+A T C_{m}}$. Such a module is likely to need a relatively long time to achieve its required ATC. Consequently, it is likely that at the later stages of phase 2 this module remains alone. This implies that the interleaving opportunities for TAM access will be reduced. Consequently TAM utilization may decrease and test application time may increase. A small value, $\epsilon$, is added to the denominator in order to prevent numerical problems when ATC is zero (e.g., at the beginning of phase 2, if there has not been any normal test).

The test application time for the schedules generated by this on-the-fly approach depends on $\theta_{m}^{L}$ and $\theta_{m}^{H}$. These temperature levels could assume a range of values provided that $\theta_{m}^{\text {stray }} \leq \theta_{m}^{L}<\theta_{m}^{H}<\theta^{\text {overheating. The }}$ temperature that corresponds to the stray power is called stray temperature and is denoted by $\theta_{m}^{\text {stray }}$ (always $\theta^{\text {ambient }} \leq \theta_{m}^{\text {stray }}<\theta^{\text {overheating }}$ ). Temperature of a module cannot be lower than this because of the stray power dissipation.

The combination of these temperature levels $\left(\theta_{m}^{L}\right.$ and $\left.\theta_{m}^{H}\right)$ among different modules affects the test application time. The proper values for these decision variables will be found in an external optimization loop, as shown in Figure 6.4.1. In the inner scheduling loop, the temperature levels (i.e., decision variables) defined by the outer optimization loop are used to generate the schedule. In Figure 6.4.1, the scheduler boxes inside the dashed box represent multiple copies of the inner scheduling algorithm. However only one of such schedulers is sufficient to perform the optimization, multiple of them are used in parallel to speed up the procedure. 
The outer optimization loop in Figure 6.4.1 makes use of a particle swarm optimization algorithm. PSO is an iterative population-based optimization metaheuristic, as discussed in section 2.7. For each alternative solution in the PSO's population, an on-the-fly scheduling is performed (inside the dashed box in Figure 6.4.1) to compute the cost function (i.e., TAT).

The working of the PSO algorithm is repeated here for convenience. The algorithm starts from a random initial population, similar to other population based metaheuristics (e.g., evolutionary methods). The population is referred to as a swarm in PSO terms. An individual in the population is referred to as a particle. Each particle goes through a number of alternative solutions, one at a time, as the algorithm iterates.

Each particle has a location in the search space (i.e., the current alternative solution). A particle records the best solution it has ever encountered, the local best. The swarm records the best solution its particles have ever encountered, the global best. Based on these best solutions and the previous alternative solution a velocity is determined which also incorporates some randomization. Velocity is the vector that determines the next location for a particle. The particles move throughout the search space in a guided random manner until they gather around a near optimal solution.

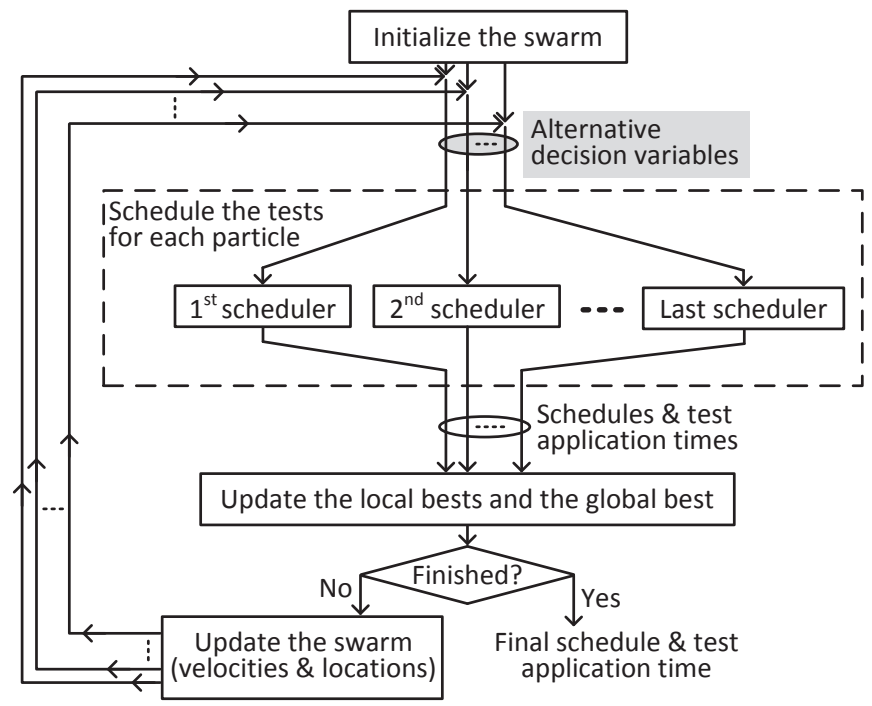

Figure 6.4.1 External optimization loop based on particle swarm optimization

The algorithm is used to minimize the test application time. Inside the dashed box, copies of the scheduling heuristics are performed in parallel for a number of particles 


\subsection{Integrated Approach}

Let us assume, now, that the orders in which normal test nodes (e.g., nodes marked with $\mathrm{N}$ in Figure 6.3.2) must be visited are given. Furthermore, assume that the order for heating sequence nodes (e.g., nodes marked with $\mathrm{H}$ in Figure 6.3.2) are also given. This means that the original test graph is broken down into a number of sub-graphs. This includes two separate directed path-graphs, one for normal tests and the other for the heating sequences among other sub-graphs.

This simplified scenario which involves two separate path-graphs will be discussed first and a path-graph scheduling algorithm will be introduced in sections 6.5.1-3. Afterwards, section 6.5.4 explains how to employ this path-graph scheduling algorithm to solve the original problem that involves the original test graph (i.e., the problem described in section 6.3).

An example in the following paragraphs, using Figure 6.5.1, explains how the proposed schedule generation works. Figure 6.5.2 shows how different blocks of the algorithm are put together. The example in Figure 6.5.1 explains how all these blocks work together to generate a schedule. Let us assume that path-graph scheduling (i.e., Path-graph scheduling block in Figure 6.5.2) determines that the module $m_{0}$ must receive heating at $i_{0}$ test cycle. Test cycles are shown in Figure 6.5.1f. It asks test graph node ordering (i.e., the node ordering block in Figure 6.5.2) for options.

Test graph ordering replies by two options (as shown in Figure 6.5.1d): The first option is $\left[s_{0,0}, s_{0,2}\right]$ that is a path-graph consisting of high power normal test nodes. The second option is $\left[s_{0,4}, s_{0,6}\right]$ that consists of heating nodes. This interaction is depicted in Figure 6.5.2 as the loop between the path-graph scheduling block and the node ordering block. The output of the node ordering block is monitored to determine if all tests are completed.

The path-graph scheduling decides to go on with $\left[s_{0,0}, s_{0,2}\right]$. Now, the power values are known and temperature simulation is performed to obtain the temperatures. This interaction is depicted in Figure 6.5.2 as the loop between the path-graph scheduling block and the temperature simulator block.

The simulated temperatures are plotted in Figure 6.5.1 a-b. As module $m_{0}$ heats up, module $m_{1}$ is slightly warmed up by the transferred heat from $m_{0}$. It is assumed that the die in this example consists of only two modules. 
Moreover, it is assumed that the test access mechanism provides access to only one of the modules at a time. The module that occupies the TAM is depicted in Figure 6.5.1c.

Every decision (i.e., change in the schedule) is recorded in the schedule as a new entry. Each entry consists of the corresponding cycle in addition to the node and state for each and every module. For example, a decision was made at cycle $i_{0}$ to start $s_{0,0}$. This is registered in the schedule as shown in Figure 6.5.1f-j. Applying $s_{0,0}$ continues smoothly to the end and then $s_{0,2}$ starts (at $i_{1}$ ), as previously suggested by the node ordering block.

At cycle $i_{2}$ the temperature of $m_{0}$ reaches the high level and cooling is required. The node ordering block is consulted and it returns $\left[s_{0,8}, s_{0,7}\right]$ that consists of low power normal tests. The other alternative is a pause

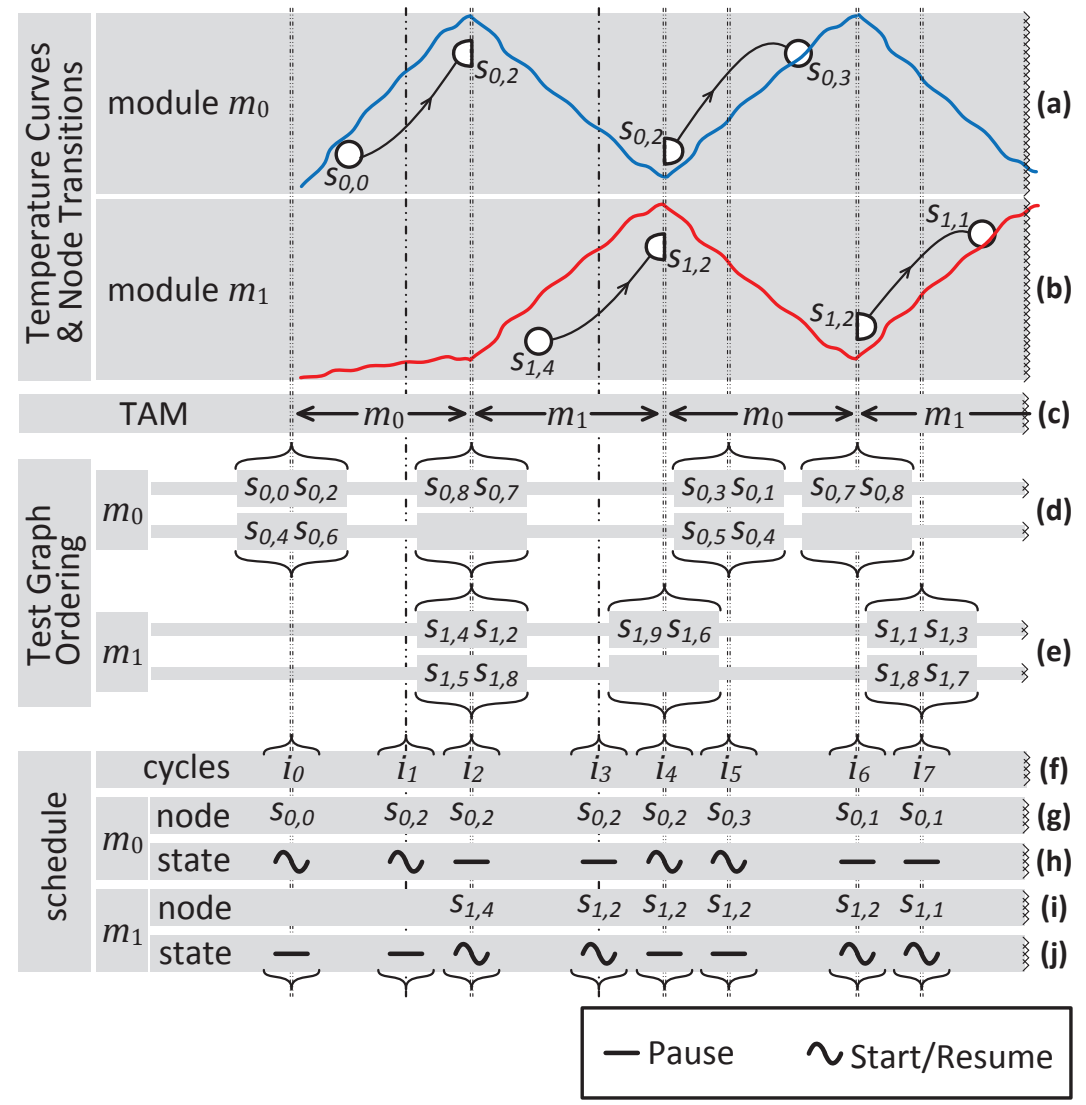

Figure 6.5.1 An example for schedule generation

(Curves are illustrative). 
(cooling interval). Since the application of $s_{0,2}$ is not complete, the application of low power normal tests is not possible. Therefore, a cooling interval is introduced. This frees the TAM that the other module can utilize. The node ordering block suggests either $\left[s_{1,4}, s_{1,2}\right]$ or $\left[s_{1,5}, s_{1,8}\right]$. The scheduler decides to go with $s_{1,4}$, a new entry for the $i_{2}$ cycle is added to the schedule and then the simulations and scheduling continue. Note that if the temperature reaches the overheating limit (that is higher than the high level discussed here and, therefore, is not shown in Figure 6.5.1) only a pause can be selected (definitely not a low power test).

At cycle $i_{4}$ the temperature of $m_{1}$ reaches the high level and cooling is required. The node ordering block is consulted and it returns $\left[s_{1,9}, s_{1,6}\right]$ that consists of low power normal tests. The other alternative, as always for cooling, is a pause. Since the application of $s_{1,2}$ is not complete, the application of low power normal tests is not possible. Therefore, a cooling interval is introduced. This frees the TAM that the other module can utilize.

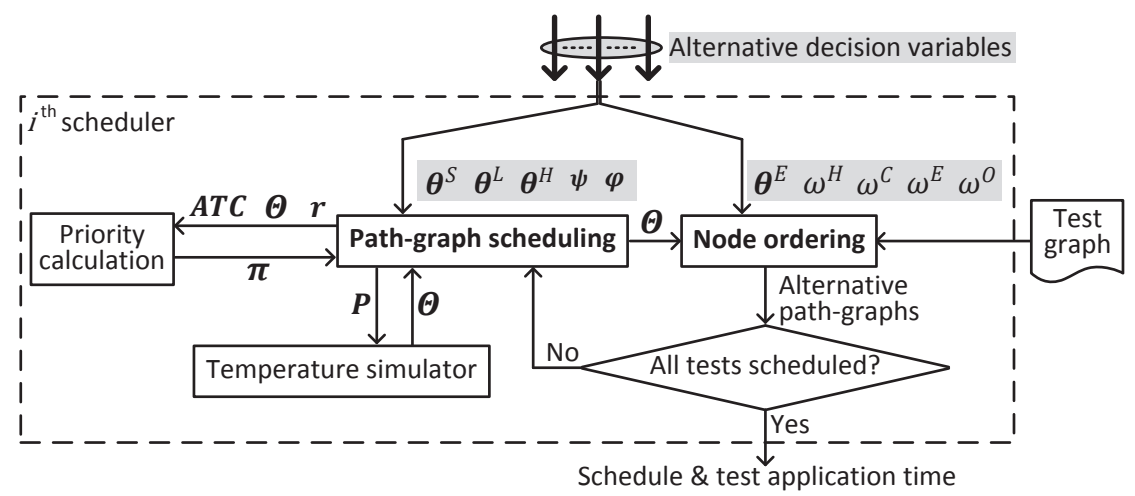

\begin{tabular}{|c|c|}
\hline $\begin{array}{c}\boldsymbol{A T C} \begin{array}{l}\text { Current amounts of } \\
\text { temperature cycling }\end{array} \\
\boldsymbol{\Theta} \text { Temperatures }\end{array}$ & $\begin{array}{ll}\boldsymbol{r} & \text { Remaining test sizes } \\
\boldsymbol{\pi} & \text { Priorities } \\
\boldsymbol{P} & \text { Power values }\end{array}$ \\
\hline \multicolumn{2}{|l|}{ Decision variables: } \\
\hline $\begin{array}{ll}\boldsymbol{\theta}^{S} & \text { Stop cooling temperature limits } \\
\boldsymbol{\theta}^{L} & \text { Low cycling temperature limits } \\
\boldsymbol{\theta}^{H} & \text { High cycling temperature limits } \\
\boldsymbol{\theta}^{E} & \text { Emergency temperature limits } \\
\boldsymbol{\psi} & \begin{array}{l}\text { Threshold on power difference } \\
\text { to decide between heating }\end{array} \\
& \text { sequence or high-power test } \\
\text { application }\end{array}$ & $\begin{array}{l}\boldsymbol{\varphi} \text { Threshold on power difference to decide } \\
\text { between cooling interval or low-power test } \\
\text { application } \\
\text { Length of the power assessment window for node } \\
\text { ordering in: } \\
\begin{array}{l}\omega^{C} \text { Cooling situation } \\
\omega^{H} \text { Heating situation } \\
\omega^{E} \text { Thermal emergency situation } \\
\omega^{O} \text { Ordinary situation }\end{array}\end{array}$ \\
\hline
\end{tabular}

Figure 6.5.2 Integrated scheduling approach

The decision variables are highlighted with gray. 
Since $s_{0,2}$ was pending, it is resumed and there is no need to consult the node ordering block at the moment. At cycle $i_{5}$, the node ordering block is consulted and $s_{0,3}$ is selected for application.

At cycle $i_{6}$ the temperature of $m_{0}$ reaches the high level and cooling is required. The node ordering block is consulted and it returns $\left[s_{0,7}, s_{0,8}\right]$ that consists of low power normal tests. Obviously, the other alternative is a pause. This time the application of $s_{0,3}$ is complete and, therefore, $s_{0,7}$ can actually be selected. However, the path-graph scheduler decides that, in any case, a pause is better. Note that before a node is started or resumed, its validity (VaT as discussed in section 6.3) is checked. If not in the VaT list, either another alternative must be selected or the module must wait until incompatible tests are complete. The above process, as explained in Figure 6.5.1, continues until all tests are performed.

\subsubsection{Path-Graph Scheduling Algorithm}

The test application time could be reduced if normal tests (phase 1) are integrated into the temperature-cycling acceleration process (phase 2). For example, a test can be employed to heat a module and avoid an unnecessary inclusion of a heating node. It may happen that a test is not powerful enough to increase the modules' temperature to $\theta_{m}^{H}$ and yet it is beneficial to include it to partially heat the module. A heating node is introduced afterwards to rapidly increase the temperature up to $\theta_{m}^{H}$.

Similar to this heating scenario, a mixed cooling scenario is also possible. The benefit of these mixing scenarios is that although the temperature will change slowly (increasing the test application time), a part of the tests is being applied (decreasing the TAT). In a mixed cooling scenario, a low power test is introduced when the temperature must decrease to create a cycle. Albeit the decrease in the module's temperature, the temperature may not decreases to $\theta_{m}^{L}$. A cooling interval is then introduced to complete the cycle.

Assume that a high power test is being applied in a heating scenario as shown in Figure 6.5.3a. Assume that the high-power test's power for the current time interval is denoted by $P_{m}^{H T}$. This power rapidly increases the temperature at the beginning. Assume that this level of power is applied for a long time. In this case a steady state temperature equal to $\theta_{m}^{S S}$ will eventually be reached. As the current temperature approaches $\theta_{m}^{S S}$, the heating rate decreases. The derivative of the temperature (i.e., heating rate) 
is shown in the lower part of Figure 6.5.3a. When the difference between the heating-sequence's heating rate and the test's heating rate increases beyond a certain threshold (threshold ${ }^{H}$ in Figure 6.5.3a), it is time to switch to the heating sequence.

This will rapidly increase the temperature to $\theta_{m}^{H}$. The temperature caused by heating sequence (shown as the red curve in Figure 6.5.3a) introduces a heating rate much larger than that of the test. Therefore, it is better to save the rest of the tests for a time that the initial temperature is lower and the tests can offer a large heating rate. The rate of temperature change (heating rate in this case) is $\frac{d}{d t} \boldsymbol{\Theta}$. Therefore the condition on heating rate is:

$$
\frac{d}{d t} \boldsymbol{\Theta}^{H S}-\frac{d}{d t} \boldsymbol{\Theta}^{H T}>\text { threshold }{ }^{H}
$$

The temperature when the heating sequence is applied is denoted by $\boldsymbol{\Theta}^{H S}$. When the high-power test is applied, the temperature is denoted by $\boldsymbol{\Theta}^{H T}$. The heating rate can be calculated based on the current temperature and upcoming power values using equation 6.1.1:

$$
\frac{d}{d t} \boldsymbol{\Theta}^{H T}=\boldsymbol{A}^{-1} \times\left(\boldsymbol{P}^{H T}-\boldsymbol{B} \times \boldsymbol{\Theta}^{H T}\right)
$$

Combining equation 6.5.1, equation 6.5.2, and the equivalent of equation 6.5.2 written for the heating sequences (instead of high power tests in equation 6.5.2) results in:

$$
\boldsymbol{A}^{-1} \times\left[\left(\boldsymbol{P}^{H S}-\boldsymbol{B} \times \boldsymbol{\Theta}^{H S}\right)-\left(\boldsymbol{P}^{H T}-\boldsymbol{B} \times \boldsymbol{\Theta}^{H T}\right)\right]>\text { threshold }^{H}
$$

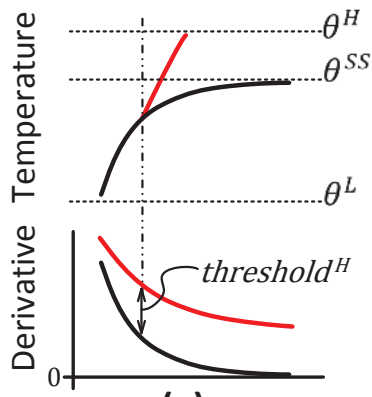

(a)

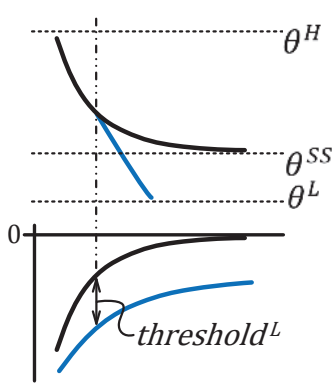

(b)

Figure 6.5.3 Thresholds in the integrated approach (a) Heating and (b) Cooling. 
Considering the fact that at the moment of decision making, there is only one actual temperature, $\boldsymbol{\Theta}\left(\boldsymbol{\Theta}=\boldsymbol{\Theta}^{H S}=\boldsymbol{\Theta}^{H T}\right)$, the condition can be further simplified to:

$$
\boldsymbol{A}^{-1} \times\left(\boldsymbol{P}^{H S}-\boldsymbol{P}^{H T}\right)>\text { threshold }^{H}
$$

This could be re-written to have the condition expressed for the power values:

$$
\left(\boldsymbol{P}^{H S}-\boldsymbol{P}^{H T}\right)>\boldsymbol{A} \times \text { threshold }^{H}
$$

Renaming $\left(\boldsymbol{A} \times\right.$ threshold $\left.^{H}\right)$ to $\boldsymbol{\psi}$ results in:

$$
\boldsymbol{P}^{H S}-\boldsymbol{P}^{H T}>\boldsymbol{\psi}
$$

Similarly, for the situation that the temperature must decrease (as shown in Figure 6.5.3b), the proper condition for switching from a test to a cooling interval is:

$$
\boldsymbol{P}^{L T}-\widehat{\boldsymbol{P}}>\boldsymbol{\varphi}
$$

The power of the low-power test is denoted by $\boldsymbol{P}^{L T}$ and the power of the cooling interval (i.e., the stray power) is denoted by $\widehat{\boldsymbol{P}}$. Switching to the cooling interval when indicated by the above equation speeds up the cooling. This way, the normal tests are employed in an efficient way during temperature-cycling process so that the overall test application time is further reduced.

According to equations $6.5 .6 \mathrm{a}-\mathrm{b}$, the scheduling heuristic does not need to compute the derivatives of the upcoming tests' temperatures. Instead, it is sufficient to compare the upcoming power values. Whenever the inequality in equation 6.5.6a is satisfied, test nodes are followed by heating nodes and whenever the inequality in equation $6.5 .6 \mathrm{~b}$ is satisfied, the testing is paused for cooling purpose. The variables $\psi_{m}$ and $\varphi_{m}$ (elements that construct $\boldsymbol{\psi}$ and $\boldsymbol{\varphi}$ vectors), are to be optimized along with $\theta_{m}^{H}$ and $\theta_{m}^{L}$, in the outer optimization loop (e.g., Figure 6.4.1), to achieve a short test application time.

These variables are optimized using PSO similar to the one explained in section 6.4. As mentioned before, Figure 6.5.2 shows how the components of the integrated approach (excluding the outer PSO loop that is already explained in Figure 6.4.1) are put together. The path-graph scheduling is shown in Figure 6.5.2 as a part of the scheduling algorithm. Other 
components of Figure 6.5.2 will be explained in the upcoming sections. Since the optimization process is similar to the PSO discussed in section 6.4, Figure 6.5.2, as a whole, can be viewed as one of the scheduler boxes shown inside the dashed box in Figure 6.4.1. The alternative decision variables shown in gray above Figure 6.5.2 come from Figure 6.4.1.

\subsubsection{Length of the Power Averaging Window}

The average upcoming powers (i.e., $\boldsymbol{P}^{L T}, \boldsymbol{P}^{H S}$, and $\boldsymbol{P}^{H T}$ ) can be calculated for a short segment of the tests or heating sequences that immediately follows. The shortest length of this segment is denoted by $\lambda_{m}$ for module $m$. Having a much shorter segment than $\lambda_{m}$ results in higher computational effort without a significant improvement in the accuracy. Taking multiple $\lambda_{m} \mathrm{~s}$ into account helps to obtain a long-term estimate of the power values. A much longer minimal segment length than $\lambda_{m}$ is not desirable since a more accurate estimate becomes unlikely to achieve.

The proper value of $\lambda_{m}$ depends on the dynamics of the system. Consider a $\lambda_{m}$ that corresponds to $100 x$ percent $(0<x<1)$ of the final response to a step input. Here, the final response is the steady state temperature and the step input is when zero input power is followed by a constant power. Assuming a constant power, the temperature equation in the time-domain can be written according to equations 6.1.2-3. We assume that the step response starts from the initial temperature equal to zero $\left(\boldsymbol{\theta}^{0}=\mathbf{0}\right)$. Replacing $\boldsymbol{\theta}^{\tau}$ with the $100 x$ percent of the final temperature results in:

$$
x \times \boldsymbol{\theta}^{S S}=\boldsymbol{\beta}(t) \times \boldsymbol{P}
$$

Since the steady state situation means negligible variations in the temperature, the temperature derivative can be assumed zero $\left(\frac{d}{d t} \boldsymbol{\theta}^{S S}=\mathbf{0}\right)$. By combining this observation with equation 6.1.1, the steady state temperature can be described as:

$$
\boldsymbol{\theta}^{S S}=\boldsymbol{B}^{-1} \times \boldsymbol{P}
$$

Replacing $\boldsymbol{\theta}^{S S}$ from the above equation and $\boldsymbol{\beta}$ from equation $6.1 .3 \mathrm{~b}$ in equation 6.5.7 results in:

$$
x \times \boldsymbol{I} \times \boldsymbol{B}^{-1} \times \boldsymbol{P}=(\boldsymbol{I}-\boldsymbol{\alpha}(\tau)) \times \boldsymbol{B}^{-1} \times \boldsymbol{P}
$$

Here we are going to replace a scalar time, $\tau$, with a matrix of time, $\boldsymbol{\Lambda}$. Besides, we assume that the equivalence of the sides in the above equation 
is achieved by satisfying the following equation (equation 6.5.10). These assumptions work for estimating the values of $\lambda_{m}$ 's [Lin84].

$$
x \times I=I-\alpha(\Lambda)
$$

Replacing $\boldsymbol{\alpha}$ from equation 6.1.3a results in

$$
\exp \left(-\boldsymbol{A}^{-1} \times \boldsymbol{B} \times \boldsymbol{\Lambda}\right)=(1-x) \times \boldsymbol{I}
$$

And finally

$$
\boldsymbol{\Lambda}=\boldsymbol{B}^{-1} \times \boldsymbol{A} \times \ln (1 /(1-x))
$$

$\left(\boldsymbol{B}^{-1} \times \boldsymbol{A}\right)$ is the time constants matrix [Lin84] (analogous to $R C$ in equations $6.1 .4,6.2 .2-6$, and 6.2 .9 for a single-element case) and $\boldsymbol{\Lambda}$ is the matrix that contains the values of $\lambda_{m}$ s. A diagonal element in $\boldsymbol{\Lambda}$ (i.e., $\lambda_{m, m}$ that is denoted by $\lambda_{m}$ ) represents the proper minimal length for averaging the upcoming test powers for module $m$.

A $\lambda_{m}$ 's value obtained this way is not too short and will contain the required information. On the other hand, the use of such $\lambda_{m}$ values prevents the temperature changes that are larger than $x \times \theta_{m}^{S S}$ from going unnoticed. This percentage, $x$, is only used for estimating the upcoming tests' average powers. The temperature simulations are always performed based on the original power sequence. Therefore, the value of $x$ will not affect them.

A set of experiments reported in [Aghaee15a] evaluate the accuracy of $\lambda_{m}$ values estimated using equation 6.5.12. The accurate value for $\lambda_{m}$ is obtained based on high quality temperature simulations. The average error is found to be around $5 \%$. This confirms that the above estimates have sufficient accuracy, in practice.

\subsubsection{Priorities for TAM Access}

Normal tests, heating sequences, and cycling tests may compete for access to TAM. The priority for letting module $m$ to access TAM is assigned based on the following criterion.

$$
\pi_{m}=\left(\theta_{m}^{L}-\theta_{m}\right) \times \frac{A T C_{m}^{R}}{\epsilon+A T C_{m}} \times r_{m}
$$

Similar to equation 6.4.1, the priority is higher for the colder modules and for the modules with larger remaining ATC. Moreover, a module's priority 
is higher if its current amount of remaining tests (denoted by $r_{m}$ ) is larger. Both normal and cycling tests are taken into account for $r_{m}$ calculation. The motivation for inclusion of $r_{m}$, similar to that of $A T C_{m}$, is to avoid a small number of modules running long after all other modules have completed their tests. Such a scenario implies inefficient use of TAM due to lack of interleaving opportunities.

In the above equation, $\theta_{m}^{L}$ is used to calculate the priority for a module running a heating sequence. For normal or cycling tests, instead of $\theta_{m}^{L}, \theta_{m}^{S}$ that indicates sufficient cooling, as introduced in section 2.7, is used in equation 6.5.13. In case of the cycling tests, $\frac{A T C_{m}^{R}}{\epsilon+A T C_{m}}$ is replaced with one (removed from equation 6.5.13) since the value of ATC is not relevant anymore (after the required ATC is achieved). The priorities are calculated based on frequently updated values for amount of temperature cycling, temperatures, and the size of the remaining tests. These values are sent out from the path-graph scheduling box in Figure 6.5.2 and the resulted priorities are sent back to it.

\subsubsection{Node Ordering in the Test Graph}

The path-graph scheduling algorithm cannot be directly employed to solve the problem that involves the original test graph (e.g., Figure 6.3.2). The path-graph scheduling needs to know, at certain time points, the order of the test nodes that will follow and sometimes also the order of the heating nodes. A path-graph format is usually used to represent the node order for different sub-graphs. These orders may change during the scheduling, as different nodes are being included in the schedule's final path-graph.

A node ordering technique is introduced in this section to determine the proper node orders, over and over again, during the scheduling process. This node ordering technique, put together with the path-graph scheduling algorithm (section 6.5.1), solves the problem that involves the original test graph, as shown in Figure 6.5.2.

For example, consider a test sub-graph with three normal and three heating nodes, as shown in Figure 6.5.4a. The graph is simplified for the situation in which the required ATC is not achieved yet. Therefore, all cycling tests can be safely removed from the original test graph, for the moment. Assume that the node $s_{0,0}$ (a normal test) is already included in the 
schedule. Assume that after $s_{0,0}$ completion, the temperature must increase in order to create a cycle.

The path-graph scheduling only needs to know what the sequence of the normal test nodes (e.g., $\left[s_{0,2}, s_{0,1}\right]$ in Figure 6.5.4b) would be if it decides to continue the schedule with the high power tests. Furthermore, it needs to know what the sequence of the heating nodes (e.g., $\left[s_{0,4}, s_{0,3}, s_{0,5}\right]$ in Figure 6.5.4b) would be if it decides to continue with the heating sequences. Based on the average power of these upcoming tests and heating sequences, the path-graph scheduling algorithm decides which node to include in the schedule, next.

For the above heating case, the high power orders for tests and heating nodes are desirable. Similar to the above heating scenario, a node ordering is performed also for the cooling scenario. In this case, there are no heating nodes, and a low power order for the test nodes is the only thing to be determined.

Let us continue with the test ordering for a heating scenario. Assume that just one node can be considered at a time to determine the high power order. Continuing with the previous example where $s_{0,0}$ is already selected, if $\overline{p_{0,0-1}}$ is larger than $\overline{p_{0,0-2}}$, then $s_{0,1}$ is selected to immediately follow $s_{0,0}$. Since only one node is left, the node ordering for the test nodes must be $\left[s_{0,1}, s_{0,2}\right]$.

Instead of only one node at a time, two nodes at a time, also, can be considered to determine the high power order. In this case, the decision is made based on a normalized power value for two nodes. For example if

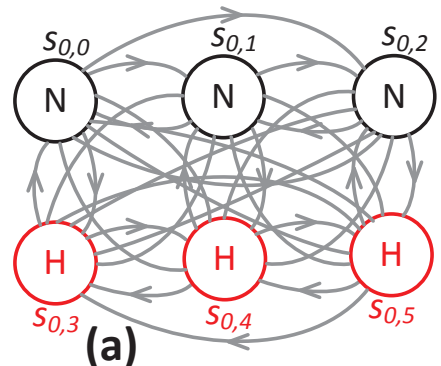

(a)

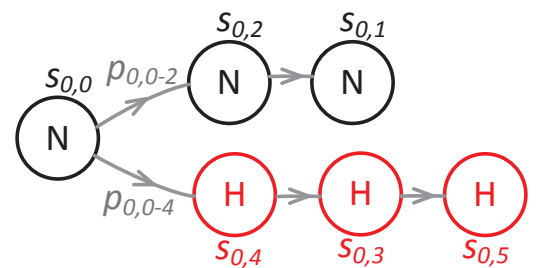

(b)

Figure 6.5.4 Node ordering

(a) Original graph (b) Ordered during the scheduling process for the time point that comes just after $S_{0,0}$ has been scheduled. 


$$
\frac{\left(\overline{p_{0,0-2}}+\overline{p_{0,2-1}}\right)}{2}>\frac{\left(\overline{p_{0,0-1}}+\overline{p_{0,1-2}}\right)}{2}
$$

Then, the node ordering for the test nodes must be $\left[s_{0,2}, s_{0,1}\right]$. The normalized power value, if node $s_{0,2}$ follows node $s_{0,1}$, is denoted by $P_{1,2}^{0}$. Therefore, in the above example, $P_{2,1}^{0}>P_{1,2}^{0}$. The number of nodes taken into account at a time could be larger. Moreover, it might be helpful to consider only a part of the test sequence at the beginning of a node.

Therefore, the ordering criterion can be generalized to consider the power values inside a power assessment window. The length of the power assessment window is defined as multiples of $\lambda_{m}$. The multiplier is denoted by $\omega^{H}$ and it is sufficient to capture the window length. Thus, the length of the power assessment window is $\omega^{H} \times \lambda_{m}$ cycles (of high-power test or heating sequence). Assume that a node consists of $\eta$ samples and $\omega^{H} \times \lambda_{m}$ cycles is equal to $v$ nodes plus $\kappa$ cycles $\left(\omega^{H} \times \lambda_{m}=v \times \eta+\kappa\right)$. This means that $\chi$ nodes $(\chi=v+1)$ will be involved. Assuming $\left[s_{0,1}, s_{0,2}, \ldots\right.$, $\left.s_{0, v}, s_{0, \chi}\right]$ as the supposed node order, its normalized power is:

$$
P_{1,2, \ldots, \chi}^{m}=\frac{\eta \times \sum_{i=1}^{v} \overline{p_{m, J-l}}}{\omega^{H} \times \lambda_{m}}+\frac{\sum_{k=0}^{\kappa-1} p_{m, v-\chi}(k)}{\omega^{H} \times \lambda_{m}}
$$

It is assumed that node $i$ is visited immediately after node $j(j=i-1$ for $\left.P_{1,2, \ldots, \chi}^{m}\right)$. Note that unlike the test nodes that are visited only once, the heating nodes may be repeated as needed. For example the heating nodes could have $\left[s_{0,3}, s_{0,3}, s_{0,3}\right]$ as the order, although this has not happened in Figure 6.5.4b. Similar to $\omega^{H}$, which is for heating situations, $\omega^{C}$ is used for the cooling situations.

A small $\omega^{H}\left(\omega^{C}\right)$ results in fast schedule generation, but the generated schedules might not be as short as they would have been with a large $\omega^{H}\left(\omega^{C}\right)$. A large $\omega^{H}\left(\omega^{C}\right)$, on the other hand, results in a slow schedule generation. Moreover, a too large $\omega^{H}$ may delay the use of some of the best heating sequences so much so that they are left unused at the end. The proper $\omega^{H}$ and $\omega^{C}$ values are obtained in the external optimization loop. In the inner ordering/scheduling loop, the $\omega^{H}\left(\omega^{C}\right)$ values defined by the outer optimization loop are used to generate the orders and the schedule as shown in Figure 6.5.2. The outer optimization loop consists of a particle swarm optimization algorithm as previously described in Figure 6.4.1. 
After the required amount of cycling is achieved, the remaining normal tests and cycling tests must be performed. In this case, heating nodes as well as the already applied normal tests can be safely removed from the original test graph. The newly created test sub-graph must be converted to a path-graph whenever the path-graph scheduling algorithm (detailed in section 6.5.1) demands a new node.

The module temperature may be high due to its previous activities or because of a high temperature in adjacent modules (heat transfer among modules). When the module's temperature is too high and close to the overheating limit ( $\left.\theta^{\text {overheating }}\right)$, it might be helpful to find a node ordering that swiftly reduces the power. This is important in a short-time window and moreover usually a rather low-power test sequence may be found if the power-assessment window is rather short.

It might not matter if this node order results in a higher test power some time later, since then the module might be cold. In such an emergency situation a short power-assessment window, denoted by $\omega^{E}$, is used. There is an emergency situation if the current temperature is larger than the emergency temperature limit, denoted by $\theta^{E}$. If the temperature is less than $\theta^{E}$ then the situation is ordinary. In any case, the nodes must be ordered in a low power configuration as detailed above.

The length of power-assessment window in this ordinary situation is denoted by $\omega^{O}$. It might be helpful to have a long ordinary powerassessment window $\left(\omega^{O}>\omega^{E}\right)$ to avoid large switching activities in a long-term sense (as opposed to short-term low-power in emergency situations). The value of $\theta_{m}^{E}$ is optimized in the outer optimization loop along with $\omega^{H}, \omega^{C}, \omega^{E}, \omega^{O}, \psi_{m}, \varphi_{m}, \theta_{m}^{S}, \theta_{m}^{L}$, and $\theta_{m}^{H}$. This outer optimization loop is similar to Figure 6.4.1 and sends the alternative decision variables to the integrated scheduling algorithm, as shown in Figure 6.5.2. The alternative decision variables shown coming to Figure 6.5.2 are from Figure 6.4.1. The generated schedule and its corresponding TAT shown going out of Figure 6.5.2 are used in Figure 6.4.1.

The search to find the best order (e.g., a path in a graph similar to Figure 6.5.4) is performed using a branch and bound approach which searches the graph down to a depth equal to $\omega^{H} \times \lambda$ cycles (also $\omega^{C}, \omega^{E}$, or $\omega^{O}$, correspondingly). The cost function (normalized power similar to equation 6.5.14) can be replaced with the accumulated power since all the 
alternatives have the same $\omega^{H}\left(\omega^{C}, \omega^{E}\right.$, or $\omega^{O}$, correspondingly). When a low power order is required (corresponding to the situation in which $\omega^{C}$, $\omega^{E}$, or $\omega^{O}$ are used), the search can be very fast, since after a relatively good path is found, the bad candidates' accumulated power values rapidly exceed the already found relatively small power value. Consequently, the inferior candidate paths are rapidly discarded. The search for heating situations may take longer but, nevertheless, the overall schedule generation procedure is adequately fast.

\subsubsection{Remarks}

As mentioned before, the proposed techniques are designed so that a test dependency graph can be accommodated. In many other cases, the test dependencies rule out some of the combinations in the schedule and therefore reduce the search space, which helps to achieve a faster schedule generation (shorter CPU time). In the case of test ordering, especially for the test graph, test dependencies will remove some of the edges in the test graph. Therefore techniques that utilize the fact that the test graph is a complete graph are not helpful in this case.

The proposed technique uses temperature simulations in order to generate a test schedule that has certain temperature characteristics. We are using a good simulator and, therefore, there is no large temperature error. Since the error is minor, a safety margin is sufficient to prevent overheating, as discussed in chapter 4 .

To ensure that a sufficient amount of cycling has been applied before the related tests, a slightly larger amount of required cycling can be assumed.

It is assumed that a node in the test graph can be paused and resumed. This is required for on-demand cooling as well as partitioning and interleaving. In other words a session-less testing scheme is used. A certain module can pause and resume its test but it cannot change to a different node before it completes the node that it has already started.

A node in a test graph may consist of a single test vector or a number of vectors that are applied one after the other. In general, a node in the test graph consists of a single test vector and, therefore, the test graph is large. Albeit the test-graphs' large sizes, the scheduling heuristic is capable of handling them, since it is very fast. However, if the number of test vectors is excessively large, then the schedule generation may become slow. In such a situation, multiple test vectors can be grouped into a single node in 
the test graph. Ideally, nodes such that their different orders do not cause large power dissipation differences should be grouped together. This reduces both the computational effort and the loss of ordering effectiveness. The test-vector clustering problem (i.e., how to group the test vectors into the nodes of a test graph) is, however, outside the scope of this thesis.

It should also be mentioned that there can be scenarios such that using a chamber-based technique is required. For example, after the packaging, to perform cycling tests targeting the IC features that are external to dies, a chamber-based technique is required.

A chamber-based approach enforces, however, the maximal cycling acceleration on all modules. This may lead to longer overall test time and unnecessary aging of modules that require less cycling acceleration. The integrated approach, on the other hand, can be faster and cheaper than the chamber-based approach. Moreover, it supports different amounts of temperature cycling for different modules. For example, one module can receive very little cycling acceleration, while another module receives a very large cycling acceleration, as needed.

\subsection{Experimental Results}

Experiments have been performed to demonstrate that the proposed technique can efficiently achieve desired temperature-cycling accelerations. Moreover, it is demonstrated that the proposed integrated approach offers a smaller test application time and, therefore, outperforms the three-phase approach. However, if the normal or cycling test schedules provided by a third-party have to be used, the three-phase approach must be chosen. In the following, first the cycling acceleration effect is demonstrated in section 6.6.1 and then, in section 6.6.2, the performance of the proposed approach is discussed.

\subsubsection{Cycling Acceleration}

The proposed integrated approach is used to perform tests and cycling acceleration for an IC with two modules, as a demonstrator example. It is assumed that the TAM in this example can only support one module to be tested at a time $(W=1)$. The corresponding temperature curves are plotted in Figure 6.6.1a. At the beginning (before 20ms) there are many normal 
tests that are properly mixed with heating sequences and cooling intervals in order to create a high cycling rate. As time goes on, the number of normal tests that can be effectively used reduces and, therefore, the majority of cycling is generated by a mix of heating sequences and cooling intervals (which is, in general, faster and more effective). Around $100 \mathrm{~ms}$, the required amounts of temperature cycling for the two modules are met and the cycling tests as well as the remaining normal tests can be applied until all tests are performed (around $110 \mathrm{~ms}$ ).

As more and more temperature cycles are performed (as in Figure 6.6.1a), the amount of temperature cycling accumulates as suggested by the increasing accelerated time in Figure 6.6.1b. The vertical axis in Figure 6.6.1b is the accelerated cycling time and the horizontal axis is the actual time. Moreover, the temperature curves in Figure 6.6.1a are used to

(a)

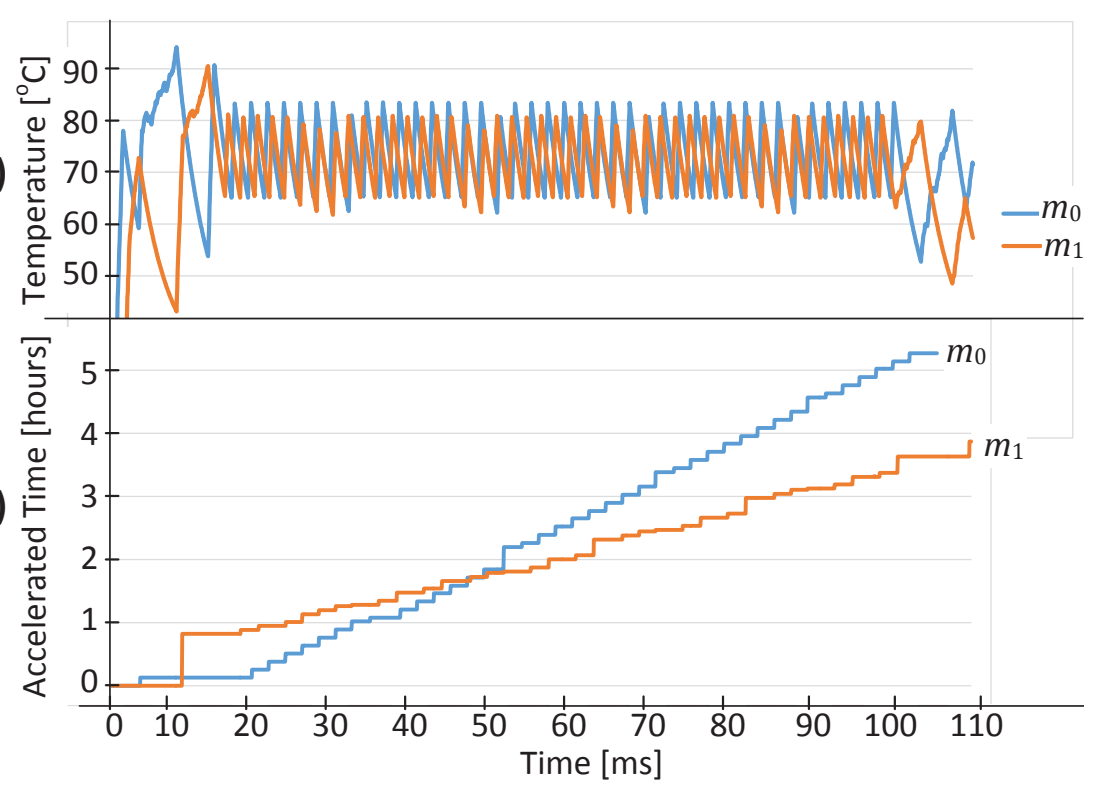

(b)

(c)

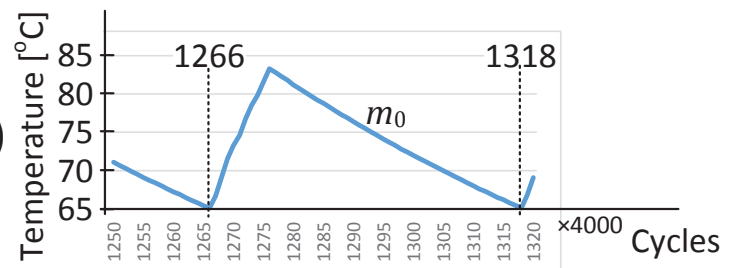

Figure 6.6.1 Cycling acceleration 
compare the proposed integrated approach (Alternative 1, below) with a chamber-based technique (Alternative 2):

\section{Alternative 1}

Let us evaluate our proposed integrated approach here. A middle section of the temperature curve from Figure 6.6.1a is magnified in Figure 6.6.1c for module $m_{0}$. Temperature swings between $65^{\circ} \mathrm{C}$ and $83^{\circ} \mathrm{C}$, resulting in a temperature-cycle amplitude equal to $18^{\circ} \mathrm{C}\left(\Delta \theta_{m}=18^{\circ} \mathrm{C}\right)$. The average temperature is approximately $74^{\circ} \mathrm{C}\left(\overline{\theta_{m}} \cong 74^{\circ} \mathrm{C}\right)$. A temperature cycle happens in $(1318-1266) \times 4000$ test cycles. The test is performed at 100MHz. Therefore:

$$
\text { cycle rate } \cong \frac{1}{(1318-1266) \times 4000 /\left(100 \times 10^{6}\right)}=\frac{1}{2.08 \times 10^{-3}} .
$$

Equation 6.1.5 should be used to calculate the ATC value. Here we assume that $\frac{1}{k_{0}} \cong 10^{-6}, k_{1} \cong 1, \theta^{\epsilon} \cong 0, \gamma \cong 4$, and $\frac{1}{k_{2}} \cong 1.07 \times 10^{-4}$. Therefore, the ATC value achieved in a second is:

$$
A T C^{A 1} \cong \frac{1}{2.08 \times 10^{-3}} \times 10^{-6} \times(18)^{4} \times \exp \left(74 \times 1.07 \times 10^{-4}\right) \cong 50.87
$$

\section{Alternative 2}

Assume a chamber-based approach that uses Thermotron ${ }^{\circledR}$ test chamber number SE-400-15-15. According to its specifications, this chamber can create a temperature-cycle, similar to that of Alternative 1, in approximately 380 seconds. Therefore:

$$
\Delta \theta_{m}=18^{\circ} \mathrm{C} \text { and } \overline{\theta_{m}} \cong 74^{\circ} \mathrm{C} \text {, cycle rate } \cong \frac{1}{380} .
$$

The ATC value achieved in a second is:

$$
A T C^{A 2} \cong \frac{1}{380} \times 10^{-6} \times(18)^{4} \times \exp \left(74 \times 1.07 \times 10^{-4}\right) \cong 278 \times 10^{-6} .
$$

The amount of temperature cycling per second achieved by the chamberbased technique is around $278 \times 10^{-6}$ while the integrated approach achieves around 50.87. This means that our approach outperforms the chamber-based technique by a huge margin (almost 180000 times $^{3}$ ).

\footnotetext{
${ }^{3}$ Although other chamber setups may perform better, their corresponding margins will be still very large.
} 


\subsubsection{Performance of the Integrated Approach}

The proposed techniques are evaluated on a set of 24 experimental ICs as detailed in Table 6.6.1. Column 1 indicates the IC's serial numbers. These ICs have one to four stacked dies (column 2). The ICs with one layer (number 1 to 6) correspond to dies at the pre-bond test stage. The ICs with more than one layer represent a mid-bond or a post-bond test stage. Each die accommodates 2, 12, 20, 30, 42, and 49 modules resulting in 2 to 196

Table 6.6.1 Percentage changes achieved by integrated approach

\begin{tabular}{|c|c|c|c|c|c|c|}
\hline \multirow{2}{*}{\multicolumn{3}{|c|}{ IC specifications }} & \multicolumn{2}{|c|}{ Percentage change in TATs } & \multicolumn{2}{|c|}{ Percentage change in CPU times } \\
\hline & & & \multicolumn{2}{|c|}{$\begin{array}{l}\text { Integrated approach compared } \\
\text { with: }\end{array}$} & \multicolumn{2}{|c|}{$\begin{array}{l}\text { Integrated approach compared } \\
\text { with: }\end{array}$} \\
\hline & $\begin{array}{l}\text { Number } \\
\text { of layers }\end{array}$ & $\begin{array}{l}\text { Number of } \\
\text { modules }\end{array}$ & $\begin{array}{l}\text { Three-phase } \\
\text { w/o ordering }\end{array}$ & $\begin{array}{l}\text { Three-phase } \\
\text { with ordering }\end{array}$ & $\begin{array}{l}\text { Three-phase } \\
\text { w/o ordering }\end{array}$ & $\begin{array}{l}\text { Three-phase } \\
\text { with ordering }\end{array}$ \\
\hline 1 & 1 & 2 & -22.99 & -21.75 & 0.00 & 0.00 \\
\hline 2 & 1 & 12 & -17.02 & -14.81 & 0.00 & 0.00 \\
\hline 3 & 1 & 20 & -29.73 & -2.12 & 0.00 & 0.00 \\
\hline 4 & 1 & 30 & -22.24 & -5.51 & 300.00 & 300.00 \\
\hline 5 & 1 & 42 & -12.65 & -10.08 & 200.00 & 200.00 \\
\hline 6 & 1 & 49 & -33.33 & -32.19 & 500.00 & 500.00 \\
\hline 7 & 2 & 4 & -19.08 & -19.08 & 0.00 & 0.00 \\
\hline 8 & 2 & 24 & -2.13 & -2.13 & 200.00 & 200.00 \\
\hline 9 & 2 & 40 & -4.58 & -3.74 & 500.00 & 500.00 \\
\hline 10 & 2 & 60 & -8.09 & -7.34 & 350.00 & 350.00 \\
\hline 11 & 2 & 84 & -9.81 & -9.52 & 325.00 & 325.00 \\
\hline 12 & 2 & 98 & -7.11 & -2.94 & 300.00 & 300.00 \\
\hline 13 & 3 & 6 & -38.16 & -35.60 & 0.00 & 0.00 \\
\hline 14 & 3 & 36 & -32.47 & -20.71 & 400.00 & 400.00 \\
\hline 15 & 3 & 60 & -15.05 & -13.85 & 333.33 & 333.33 \\
\hline 16 & 3 & 90 & -9.034 & -8.92 & 283.33 & 283.33 \\
\hline 17 & 3 & 126 & -10.88 & -7.45 & 169.23 & 191.67 \\
\hline 18 & 3 & 147 & -22.32 & -12.98 & 83.33 & 83.33 \\
\hline 19 & 4 & 8 & -52.83 & -44.92 & 0.00 & 0.00 \\
\hline 20 & 4 & 48 & -22.12 & -21.95 & 25.00 & 25.00 \\
\hline 21 & 4 & 80 & -25.04 & -24.86 & 13.04 & 13.04 \\
\hline 22 & 4 & 120 & -16.22 & -16.03 & 18.48 & 15.96 \\
\hline 23 & 4 & 168 & -16.97 & -16.96 & 14.43 & 18.08 \\
\hline \multirow[t]{2}{*}{24} & 4 & 196 & -22.99 & -13.99 & 20.50 & 26.87 \\
\hline & Average & & -19.70 & -15.39 & 168.15 & 169.40 \\
\hline \multicolumn{6}{|c|}{$\begin{array}{l}1 \text { Percentage change in the integrated approach's test application time }\left(T A T^{I}\right) \\
\text { compared with the 3-phase approach without test ordering }\left(T A T^{3 w 00}\right)\end{array}$} & $\frac{T A T^{I}-T A T^{3 w o O}}{T A T^{3 w o O}}$ \\
\hline \multirow{2}{*}{$\begin{array}{ll}2 \mathrm{P} \\
\mathrm{c}\end{array}$} & \multirow{2}{*}{\multicolumn{5}{|c|}{$\begin{array}{l}\text { Percentage change in the integrated approach's test application time }\left(T A T^{I}\right) \\
\text { compared with the 3-phase approach with test ordering }\left(T A T^{30}\right)\end{array}$}} & $T A T^{I}-T A T^{30}$ \\
\hline & & & & & & $T A T^{30}$ \\
\hline \multirow{2}{*}{$\begin{array}{ll}3 \mathrm{P} \\
\mathrm{n}\end{array}$} & \multirow{2}{*}{\multicolumn{5}{|c|}{$\begin{array}{l}\text { Percentage change in the integrated approach's } C P U \text { time }\left(C P U^{I}\right) \text { compared } \\
\text { with the 3-phase approach without test ordering }\left(C P U^{3 w o O}\right)\end{array}$}} & $C P U^{I}-C P U^{3 w o o}$ \\
\hline & & & & & & $C P U^{3 w o o}$ \\
\hline 4 & \multicolumn{5}{|c|}{$\begin{array}{l}\text { Percentage change in the integrated approach's } C P U \text { time }\left(C P U^{I}\right) \text { compared } \\
\text { with the 3-phase approach with test ordering }\left(C P U^{30}\right)\end{array}$} & $\frac{C P U^{I}-C P U^{30}}{C P U^{30}}$ \\
\hline
\end{tabular}


modules per IC, as shown in column 3 . The number of the corresponding nodes in the test graphs is between 60 and 5880 and test sizes are between $234 \mathrm{kB}$ and $22 \mathrm{MB}$. The thermal models are extracted using an approach similar to [Coskun09]. The switching activities for tests and heating sequences are generated using Markov chains, similar to [Yao11c]. All experiments are performed on a desktop computer with Intel ${ }^{\circledR}$ Xeon ${ }^{\circledR}$ W3520 processor and 8 GB of memory.

The required amount of cycling is separately defined for each module in the experimental ICs. It does not depend on the scheduling method and, therefore, both the three-phase and integrated approaches must enforce identical amounts of temperature cycling. Since before enforcing $A T C_{m}^{R}$, cycling tests cannot be performed, the cycling operations continue until $A T C_{m}^{R}$ is enforced. This implies that it is sufficient to compare the test application times.

The integrated approach achieves shorter TAT compared with the threephase approach for all of the experimental ICs. Two types of the threephase approach are considered. Type 1 does not use any ordering technique. Type 2 uses a test ordering technique. The percentage changes compared with the three-phase approach without test ordering (type 1) and with test ordering (type 2) are reported in columns 4 and 5, respectively. In average, the proposed technique outperforms the three-phase technique with ordering and the three-phase technique without ordering by about 15 percent and 20 percent, respectively. Since the test ordering offers a reduced TAT, the improvements achieved by the integrated approach are larger when compared with the three-phase without test-ordering approach.

Compared with the three-phase approach, the integrated approach is more complicated and, therefore, it takes more time to run. The CPU times are rounded to seconds and then percentage changes are calculated. The percentage changes in the CPU times are reported in columns 6 and 7 for the integrated approach compared with the three-phase approach without and with test ordering, respectively. In average, the proposed technique is slower than the three-phase technique with ordering and the three-phase technique without ordering by about 169 and 168 in percentage change, respectively.

The CPU times for the three-phase approaches with and without test ordering are comparable. Compared with the three-phase without ordering approach, the three-phase with ordering approach is more complicated for 
the decision-points ${ }^{4}$ in the schedule. This is due to the time taken to search for a good node order. On the other hand, the three-phase with node ordering approach offers slightly shorter schedules which mean less decision-points. Consequently, sometimes shorter schedules compensate for the time-consuming node ordering operations, but not always. Therefore, sometimes the number in columns 6 is smaller than the number in column 7, for example for IC number 17 and sometimes larger, for example for IC number 22.

Note that in these experiments for CPU times we included the scheduling times for the normal and cycling tests. If the schedules would have been provided by a third-party, then the actual CPU times for the three-phase approaches will be smaller. Consequently, the numbers reported in columns 6 and 7 could be larger than the current values. Shorter CPU time can be considered as an advantage for the three-phase approach.

CPU times, in general, grow with the tests size as shown in Figure 6.6.2. Moreover, CPU times grow also with the number of modules and layers as shown in Figure 6.6.2b. The data points in Figure 6.6.2a represent multiples of test sizes used in Figure 6.6.2b. The growth rates are however

(a)

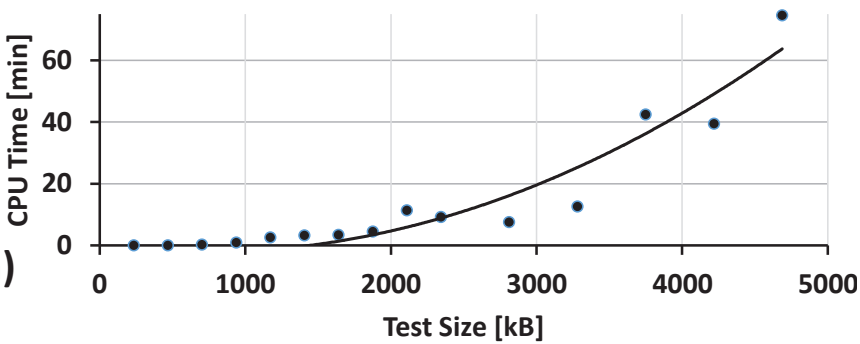

(b)

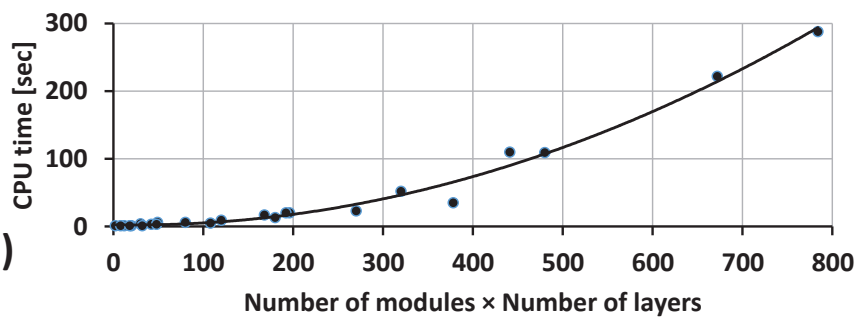

Figure 6.6.2 CPU time growth (a) with test size, (b) with IC complexity

\footnotetext{
${ }^{4} \mathrm{~A}$ decision-point is a point that a module's state (testing/heating/cooling) or test/heating node may change.
} 


\section{Chapter 6}

acceptably low and the scheduling process for the largest IC (number 24) takes less than 5 minutes to complete.

\subsection{Conclusions}

Temperature-cycling acceleration is a useful technique to help the detection of cycling-dependent early-life failures. These failures are usually not considered as a major issue for conventional 2D ICs. Therefore, cycling acceleration is usually recommended when a high degree of reliability is crucial. Recent studies have shown that the cycling-dependent early-life failures can be a major issue for 3D stacked ICs. The existing cycling acceleration procedures are very costly since they are usually performed using temperature chambers. In this thesis we propose an inexpensive technique to order the tests and heating sequences so that required temperature cycling effects can be achieved in a short time, without the use of temperature chambers.

For this purpose tests are ordered differently based on the required power for the related situation. When a module's temperature must increase to generate a temperature cycle, a high-power ordering of the tests and heating sequences is considered. For the situation that the temperature must decrease, a low-power ordering of the tests is used, instead. During the tests, after the required cycling is achieved, depending on the current module's temperature, a long term or a short term low-power ordering of the tests is selected. All these help to achieve a short test application time, as demonstrated by the experiments. Consequently, this integrated approach is well-suited to be integrated into pre-, mid-, and post-bond test stages for 3D stacked ICs. 


\subsection{Notations and Abbreviations}

\begin{tabular}{|c|c|}
\hline Notation & Description \\
\hline $\boldsymbol{A}$ & Represents heat capacitances in the thermal model \\
\hline$A T C$ & Amount of temperature cycling \\
\hline$A T C_{m}^{R}$ & Required amount of temperature cycling for module $m$ \\
\hline $\boldsymbol{B}$ & $\begin{array}{l}\text { Represents thermal conductance (related to heat transfer) in the } \\
\text { thermal model }\end{array}$ \\
\hline$C$ & Heat capacitance in a simple single module case (analogous to $\boldsymbol{A}$ ) \\
\hline$I$ & Identity matrix \\
\hline $\begin{array}{l}k_{0} \\
k_{1} \\
k_{2}\end{array}$ & Constants in ATC equation \\
\hline $\mathcal{N}_{m}$ & Number of temperature cycles for module $m$ \\
\hline $\begin{array}{l}\boldsymbol{P} \\
P\end{array}$ & Power value(s) in a general case \\
\hline$P^{H S}$ & Heating sequences' powers \\
\hline $\boldsymbol{P}^{H T}$ & High-power tests' powers \\
\hline$P^{L T}$ & Low-power tests' powers \\
\hline$\widehat{\boldsymbol{P}}$ & Stray power \\
\hline PSO & Particle Swarm Optimization [Poli07] \\
\hline$R$ & $\begin{array}{l}\text { Thermal resistance in a simple single module case (analogous to } \\
\boldsymbol{B}^{-1} \text { ) }\end{array}$ \\
\hline$r_{m}$ & Remaining tests' size \\
\hline$s_{m, n}$ & $\begin{array}{l}\text { Node } n \text { in module } m \text { 's test graph (same as } n \text {-th test for module } \\
m \text { ) }\end{array}$ \\
\hline TAM & Test Access Mechanism \\
\hline TAT & Test Application Time \\
\hline$\alpha$ & Transfer matrix for initial temperatures \\
\hline$\beta$ & Transfer matrix for power values \\
\hline$\gamma$ & Constant in ATC equation \\
\hline$\eta$ & $\begin{array}{l}\text { Number of samples/cycles in one test/heating node. } \\
\eta \times v+\kappa=\omega^{H} \times \lambda\end{array}$ \\
\hline
\end{tabular}


Chapter 6

\begin{tabular}{|c|c|}
\hline Notation & Description \\
\hline $\boldsymbol{\Theta}$ & Temperatures vector in a general case \\
\hline$\theta^{\text {ambient }}$ & Ambient temperature \\
\hline$\theta^{\text {overheating }}$ & Overheating temperature limit \\
\hline$\theta_{m}^{\text {stray }}$ & Stray temperature (caused by stray power, $\widehat{\boldsymbol{P}}$ ) \\
\hline$\Theta^{0}$ & Initial temperature(s) \\
\hline \multicolumn{2}{|l|}{$\Theta^{0}$} \\
\hline $\boldsymbol{\Theta}^{\tau}$ & Final temperature(s) after $\tau$ seconds \\
\hline \multicolumn{2}{|l|}{$\Theta^{\tau}$} \\
\hline $\boldsymbol{\theta}^{S S}$ & Steady state temperatures \\
\hline$\theta_{m}^{H}$ & High cycling temperature limit for module $m$ \\
\hline$\theta_{m}^{L}$ & Low cycling temperature limit for module $m$ \\
\hline$\theta_{m}^{E}$ & Emergency temperature limit for module $m$ \\
\hline$\theta_{m}^{S}$ & Stop cooling temperature limit for module $m$ \\
\hline$\theta^{\epsilon}$ & Threshold for temperature cycle amplitudes \\
\hline$\overline{\theta_{m}}$ & Average cycling temperature for module $m$ \\
\hline$\Delta \theta_{m}$ & The amplitude of temperature cycles for module $m$ \\
\hline$\frac{d}{d t} \boldsymbol{\Theta}^{H S}$ & Heating rate of the heating sequences \\
\hline$\frac{d}{d t} \Theta^{H T}$ & Heating rate of the high-power tests \\
\hline$\kappa$ & $\begin{array}{l}\text { Remaining number of cycles when full nodes are subtracted from } \\
\text { the length of power assessment window }\left(\omega^{H} \times \lambda\right) \text {. } \omega^{H} \times \lambda \text { covers } \\
v \text { nodes plus } \kappa \text { cycles. }\end{array}$ \\
\hline $\begin{array}{l}\Lambda \\
\lambda_{m}\end{array}$ & $\begin{array}{l}\text { Minimal segment lengths for power averaging/assessment (Vector } \\
\text { and element formats) }\end{array}$ \\
\hline$\mu$ & Average cycling temperature for single module example \\
\hline$v$ & $\begin{array}{l}\text { Number of full nodes in the power assessment window }\left(\omega^{H} \times \lambda\right) \text {. } \\
\omega^{H} \times \lambda \text { covers } v \text { nodes plus } \kappa \text { cycles. }\end{array}$ \\
\hline$\pi_{m}$ & TAM access priority for module $m$ \\
\hline
\end{tabular}


Integrated Temperature-Cycling Acceleration and Test

\begin{tabular}{ll}
\hline Notation & Description \\
\hline$\rho_{m}$ & ATC rate \\
\hline$\sigma$ & Half of temperature cycle amplitude for single module example \\
\hline$\tau$ & $\begin{array}{l}\text { Time period between the initial temperatures and the final } \\
\text { temperature }\end{array}$ \\
\hline $\boldsymbol{\varphi}$ & $\begin{array}{l}\text { Threshold on power difference to decide between cooling interval } \\
\text { or low-power test application }\end{array}$ \\
\hline$\chi$ & $\begin{array}{l}\text { Number of nodes involved in the power assessment window } \\
\left(\omega^{H} \times \lambda\right) .\end{array}$ \\
\hline $\boldsymbol{\psi}$ & $\begin{array}{l}\text { Threshold on power difference to decide between heating } \\
\text { sequence or high-power test application }\end{array}$ \\
\hline$\omega$ & $\begin{array}{l}\text { Length of the power assessment window. } \omega \times \lambda \text { cycles are } \\
\text { considered. Refers indifferently to } \omega^{C}, \omega^{E}, \omega^{H}, \text { or } \omega^{O} .\end{array}$ \\
\hline$\omega^{C}$ & $\omega$ for node ordering in Cooling situation. \\
\hline$\omega^{E}$ & $\omega$ for node ordering in thermal Emergency situation. \\
\hline$\omega^{H}$ & $\omega$ for node ordering in Heating situation. \\
\hline$\omega^{O}$ & $\omega$ for node ordering in Ordinary situation. \\
\hline
\end{tabular}





\section{Chapter 7 Conclusions and Future Work}

\subsection{Conclusions}

Many cutting-edge computer and electronic products are based on advanced Systems-on-Chip (SoC). Advanced SoCs are manufactured with deep submicron and 3D-stacked-IC technologies. These advanced manufacturing technologies enable the integration of a large number of high performance functions. Such advanced manufacturing technologies face a number of thermal challenges in regard with their reliability and testing procedures. These challenges, related to temperature uncertainty, temperature gradients, and temperature cycling have been addressed in this thesis.

\section{Temperature Uncertainty}

Advanced SoCs manufactured with deep submicron technologies suffer from process variation and its thermal consequences. Existing testing techniques rely on temperature simulations to predict the circuit-undertests' temperatures and design the test so that overheating is prevented. The difference between the expected temperatures and the actual temperatures is called temperature error. This error, for past technologies, is negligible. However, advanced SoCs experience large error magnitudes due to large process variations. Such large error magnitudes have costly consequences (e.g., test overkill and overheating) and must be taken care of.

This thesis presents several scheduling-based approaches to take care of the temperature errors induced by process variation. An adaptive technique for addressing the intra-die and time-variant errors is introduced. This technique is designed to support a thermal-safe test which means that a high-temperature limit must be respected. A slightly different scenario is multi-temperature testing which also requires considering a low- 
temperature limit. An adaptive technique to deal with temperature errors that affect multi-temperature testing is therefore proposed.

\section{Temperature Gradients}

Temperature gradients in a chip accelerate certain defect mechanisms including some types of early-life failures. Therefore, performing a burnin like operation that enforces appropriate gradients helps to accelerate and detect these early-life failures. A test-scheduling based approach for performing burn-in like operations is proposed in this thesis. The proposed approach enforces the required temperature gradients by selectively applying high power test stimuli to the circuit-under-test. This way, the required life-time acceleration is achieved without requiring temperature chambers.

Temperature gradients affect also some delay-related defects. The delay experienced by a signal depends on its path temperature. Moreover, some defects (e.g., resistive opens) can, also, affect the delay. Different signals travelling through different paths may therefore experience different delays because of a subtle defect in one of the paths as well as the path's temperature. This means that the circuit may operate correctly when the gradients are negligible even though a subtle defect exists. However, this negligible defect may cause a fault when certain gradient occurs on the chip. In order to detect such subtle defects, the related tests must be applied when appropriate temperature-gradients are enforced. 3D stacked ICs experience large gradients and, therefore, the proposed techniques are developed so that they can be efficiently applied to 3D stacked ICs.

\section{Temperature Cycling}

Temperature-cycling test procedures are usually applied to safety-critical systems to detect cycling-related early-life failures. Such failures affect advanced SoCs, particularly through-silicon-via structures in 3D-stackedICs. An efficient schedule-based cycling-test technique that combines cycling acceleration with testing is proposed in this thesis.

The circuit-under-test's dissipated power depends on the order in which the tests are applied. Therefore, the tests are reordered by the proposed technique to adjust the power dissipation levels as needed. This helps to achieve a short test application time. Moreover, the proposed technique fits into existing $3 \mathrm{D}$ testing procedures and does not require temperature 
chambers. Therefore, the overall cycling acceleration and testing cost can be drastically reduced.

\section{Temperature Simulation and Experiments}

A fast temperature simulation technique based on a closed-form solution for the temperature equations is introduced in this thesis. Dedicated experiments show that the proposed simulation technique reduces the schedule generation time by more than half. This technique is used in the majority of the experiments reported in this thesis.

All the proposed techniques in this thesis have been implemented and evaluated with extensive experiments based on ITC'02 benchmark as well as a number of experimental 3D-stacked-ICs. Experiments show that the proposed techniques work effectively and reduce the costs, in particular the costs related to addressing thermal issues and early-life failures.

\subsection{Future Work}

In this thesis we focused on the manufacturing test process. However, infield and online testing are required, for example, for safety-critical systems. Similar issues to those considered in this thesis, for manufacturing testing, can cause problems during in-field and online testing as well. Temperature issues caused by process variation and temperature gradients are among these issues.

Temperature cycling for applications that require frequent in-field or online testing is another direction for future research. Designing these testing procedures that minimize the temperature cycling can be of interest, in order to slow down the aging process. This is also true for minimizing the gradients. Utilizing the already existing gradients (during normal operation) for online gradient-based testing can be efficient and therefore interesting to study.

Adaptive online testing is another related topic. Temperature cycling and gradients can be monitored (during the normal operation) and online tests targeting the weakened areas (likely defects) can be applied. The temperature errors (caused by process variation) can, also, be estimated during the normal operation and then a decision between using a slow (lowpower) and a fast (high-power) online test scheme can be made accordingly. For example for modules that work warmer than usual a longer low-power online test might be a good choice. On the other hand, 
for modules that work colder than usual a faster high-power online test might be a good choice. This may change over time, partly in relation to gradients and cycling.

In a manufacturing test setup, testing frequency can be used to alter the power dissipation, if the DfT circuitry and the ATE support it. For example, when a colder testing is preferred, the frequency can be reduced. If heating is required, then the frequency could be increased to generate more heat. Although not used in this thesis, testing frequency can be added as another decision variable to the problem formulation.

Defect explorations and reliability studies can identify new challenging issues that need to be addressed, especially for new technologies. Many potential issues regarding through silicon vias are already identified, some of which are discussed in this thesis. As 3D stacked IC technology matures, more issues may be identified. This is, in particular, important for applications that require high reliability.

The focus of this thesis is mainly on logic, even though some of the proposed techniques can be applied to whatever entity that has the properties of a module (as discussed earlier). There exist several non-logic components that are usually integrated into advanced SoCs and similar devices. Memory modules are very important among these devices and are widely studied in connection with normal 2D and 3D-stack technologies. Process variation, temperature gradients, and temperature cycling affect the memories, too.

There are other components that are similarly affected by these negative effects. Image sensors that are widely used today are among them. CMOS image sensors can be manufactured using through silicon vias. Consequently, defects that relate to through silicon vias affect such image sensors, among other sources of defect. Developing new testing techniques as well as extending and specializing the methods proposed in this thesis can be of interest for all these non-logic components. 


\section{References}

[Abramovici94] Miron Abramovici, Melvin A Breuer, and Arthur D Friedman. DigitAL systemS TESTING AND TESTABLE DESIGN, 1994. [Online]. Available: http://eu.wiley.com/WileyCDA/WileyTitle/productCd-0780310624.html. [Accessed: 29-May-2015].

[Aghaee10] Nima Aghaee, Zhiyuan He, Zebo Peng, and Petru Eles. TemPerature-AWARE SOC TEST SCHEDULING CONSIDERING INTER-CHIP PROCESS VARIATION, in 19th Asian Test Symposium (ATS), pages 395-398, 2010.

[Aghaee11a] Nima Aghaee, Zebo Peng, and Petru Eles. AdAPtive TEMPERAture-AWARE SoC TEST SCHEDULING CONSIDERING PROCESS VARIATION, in 14th Euromicro Conference on Digital System Design (DSD), Oulu, Finland, pages 197-204, 2011.

[Aghaee11b] Nima Aghaee, Zebo Peng, and Petru Eles. Process-VARIATION AND TEMPERATURE AWARE SOC TEST SCHEDULING USING PARTICLE SWARM OPTIMIZATION, in 6th International Design and Test Workshop (IDT), Beirut, Lebanon, pages 1-6, 2011.

[Aghaee13a] Nima Aghaee, Zebo Peng, and Petru Eles. Process-Variation And TEMPERATURE AWARE SOC TEST SCHEDULING TECHNIQUE, Journal of Electronic Testing, vol. 29, no. 4, pages 499-520, Aug. 2013.

[Aghaee13b] Nima Aghaee, Zebo Peng, and Petru Eles. TemPERAtUre-GradiEnT BASED TEST SCHEDULING FOR 3D STACKED ICs, in 20th International Conference on Electronics, Circuits, and Systems (ICECS), Abu Dhabi, UAE, pages 405-408, 2013.

[Aghaee14a] Nima Aghaee, Zebo Peng, and Petru Eles. An EFFICIENT TeMPERATURE-Gradient BASED BURN-IN TECHNIQUE FOR 3D STACKED ICs, in Design, Automation and Test in Europe Conference and Exhibition (DATE), Dresden, Germany, pages 1-4, 2014.

[Aghaee14b] Nima Aghaee, Zebo Peng, and Petru Eles. Process-Variation AWARe MUltiTEMPERATURE TEST SCHEDULING, in 27th International Conference on VLSI Design, Mumbai, India, pages 32-37, 2014.

[Aghaee15a] Nima Aghaee, Zebo Peng, and Petru Eles. An integrated temperatureCYCLING ACCELERATION AND TEST TECHNIQUE FOR 3D STACKED ICS, in 20th Asia and South Pacific Design Automation Conference (ASP-DAC), Chiba, Japan, pages $526-531,2015$. 


\section{References}

[Aghaee15b] Nima Aghaee, Zebo Peng, and Petru Eles. TEMPERATURE-GRADIENT-BASED BURNIN AND TEST SCHEDULING FOR 3-D STACKED ICS, IEEE Transactions on Very Large Scale Integration (VLSI) Systems, vol. PP, no. 99, pages 1-1, 2015.

[Ahmed05] Nisar Ahmed, Mohammad Tehranipoor, and CP Ravikumar. ENHANCED LAUNCHOFF-CAPTURE TRANSITION FAULT TESTING, in International Test Conference, pages 255-264, 2005.

[Ayala09] José L Ayala, Arvind Sridhar, Vinod Pangracious, David Atienza, and Yusuf Leblebici. THROUGH SILICON VIA-BASED GRID FOR THERMAL CONTROL IN 3D CHIPS, in Nano-Net, Springer, pages 90-98, 2009.

[Bahukud08a] Sudarshan Bahukudumbi and Krishnendu Chakrabarty. POWER MANAGEMENT FOR WAFER-LEVEL TEST DURING BURN-IN, in 17th Asian Test Symposium, pages 231$236,2008$.

[Bahukud08b] Sudarshan Bahukudumbi and Krishnendu Chakrabarty. TEST-PATTERN ORDERING FOR WAFER-LEVEL TEST DURING BURN-IN, in 26th VLSI Test Symposium, pages 193-198, 2008.

[Bahukud09] Sudarshan Bahukudumbi and Krishnendu Chakrabarty. Power MANAGEMENT USING TEST-PATTERN ORDERING FOR WAFER-LEVEL TEST DURING BURN-IN, IEEE Transactions on Very Large Scale Integration (VLSI) Systems, vol. 17, no. 12, pages 1730-1741, Dec. 2009.

[Bayle10] F Bayle and A Mettas. TEMPERATURE ACCELERATION MODELS IN RELiABILITY PREDICTIONS: JUSTIFICATION AND IMPROVEMENTS, in annual Reliability and Maintainability Symposium (RAMS), pages 1-6, 2010.

[Bild08] David R Bild, Sanchit Misra, Thidapat Chantemy, Prabhat Kumar, Robert P Dick, $\mathrm{X}$ Sharon $\mathrm{Hu}$, Li Shang, and Alok Choudhary. TEMPERATURE-AWARE TEST SCHEDULING FOR MULTIPROCESSOR SYSTEMS-ON-CHIP, in IEEE/ACM International Conference on Computer-Aided Design, pages 59-66, 2008.

[Bonhomme02] Y Bonhomme, P Girard, C Landrault, and S Pravossoudovitch. TEST POWER: A BIG ISSUE IN LARGE SOC DESIGNS, in $1^{\text {st }}$ International Workshop on Electronic Design, Test and Applications, pages 447-449, 2002.

[Borkar03] Shekhar Borkar, Tanay Karnik, Siva Narendra, Jim Tschanz, Ali Keshavarzi, and Vivek De. PARAMETER VARIATIONS AND IMPACT ON CIRCUITS AND MICROARCHITECTURE, in 40th annual Design Automation Conference, pages 338$342,2003$.

[Bosio11] A Bosio, L Dilillo, P Girard, A Todri, A Virazel, K Miyase, and X Wen. PowerAWARE TEST PATTERN GENERATION FOR AT-SPEED LOS TESTING, in $20^{\text {th }}$ Asian Test Symposium, pages 506-510, 2011.

[Bota04] Sebastiàn A Bota, M Rosales, JL Rosello, A Keshavarzi, and J Segura. WiTHIN DIE THERMAL GRADIENT IMPACT ON CLOCK-SKEW: A NEW TYPE OF DELAY-FAULT MECHANISM, in International Test Conference, pages 1276-1283, 2004.

[Carbine97] Adrian Carbine and Derek Feltham. Pentium (R) Pro PROCESSOR Design FOR TEST AND DEBUG, in International Test Conference, pages 294-303, 1997.

[Chakrabarty00] Krishnendu Chakrabarty. TEST SCHEDULING FOR CORE-BASED SYSTEMS USING MIXED-INTEGER LINEAR PROGRAMMING, IEEE Transactions on Computer-Aided Design of Integrated Circuits and Systems, vol. 19, no. 10, pages 1163-1174, Oct. 2000 . 


\section{References}

[Chakrabarty02] Krishnendu Chakrabarty, Vikram Iyengar, and Anshuman Chandra. TEST SCHEDULING USING MIXED-INTEGER LINEAR PROGRAMMING, Frontiers in Electronic Testing: Test Resource Partitioning for System-on-a-Chip, Springer US, pages 97-118, 2002.

[Chakrabarty12] Krishnendu Chakrabarty, Sergej Deutsch, Himanshu Thapliyal, and Fangming Ye. TSV DEFECTS AND TSV-INDUCED CIRCUIT FAILURES: THE THIRD DIMENSION IN TEST AND DESIGN-FOR-TEST, in International Reliability Physics Symposium, page $5 \mathrm{~F}-1,2012$.

[Chakravarty94] S Chakravarty and VP Dabholkar. TWO TECHNIQUES FOR MINIMIZING POWER DISSIPATION IN SCAN CIRCUITS DURING TEST APPLICATION, in $3^{\text {rd }}$ Asian Test Symposium, pages 324-329, 1994.

[Chandran09] Unni Chandran and Dan Zhao. THERMAL DRIVEN TEST ACCESS ROUTING IN HYPERINTERCONNECTED THREE-DIMENSIONAL SYSTEM-ON-CHIP, in 24th IEEE International Symposium on Defect and Fault Tolerance in VLSI Systems, pages 410-418, 2009.

[Chang05] Hongliang Chang and Sachin S Sapatnekar. Full-CHIP ANALYSIS OF LEAKAGE POWER UNDER PROCESS VARIATIONS, INCLUDING SPATIAL CORRELATIONS, in 42nd annual Design Automation Conference, pages 523-528, 2005.

[Chantem13] Thidapat Chantem, Yun Xiang, X Sharo Hu, and Robert P Dick. EnHancing MULTICORE RELIABILITY THROUGH WEAR COMPENSATION IN ONLINE ASSIGNMENT AND SCHEDULING, in Design, Automation Test in Europe, pages 1373-1378, 2013.

[Cheng00] Kwang Ting Cheng, S Dey, M Rodgers, and K Roy. TeST CHALLENGES FOR DEEP SUB-MiCRON TECHNOLOGIES, in Design Automation Conference, pages 142-149, 2000 .

[Cherman12] VO Cherman, J De Messemaeker, K Croes, B Dimcic, G Van der Plas, I De Wolf, G Beyer, B Swinnen, and E Beyne. IMPACT OF THROUGH SILICON VIAS ON FRONTEND-OF-LINE PERFORMANCE AFTER THERMAL CYCLING AND THERMAL STORAGE, in International Reliability Physics Symposium, pages 2B.3.1-2B.3.5, 2012.

[Choi07] Jung Hwan Choi, Jayathi Murthy, and Kaushik Roy. THE EFFECT OF PROCESS VARIATION ON DEVICE TEMPERATURE IN FINFET CIRCUITS, in IEEE/ACM international conference on Computer-aided design, pages 747-751, 2007.

[Chou97] RM Chou, KK Saluja, and VD Agrawal. SCHEDULING TESTS FOR VLSI SYSTEMS UNDER POWER CONSTRAINTS, IEEE Transactions on Very Large Scale Integration (VLSI) Systems, vol. 5, no. 2, pages 175-185, Jun. 1997.

[Ciappa03a] M Ciappa, F Carbognani, P Cova, and W Fichtner. LiFETIME PREDICTION AND DESIGN OF RELIABILITY TESTS FOR HIGH-POWER DEVICES IN AUTOMOTIVE APPLICATIONS, in 41st annual International Reliability Physics Symposium, pages 523-528, 2003.

[Ciappa03b] M Ciappa, F Carbognani, and Wolfgang Fichtner. LIFETIME PREDICTION AND DESIGN OF RELIABILITY TESTS FOR HIGH-POWER DEVICES IN AUTOMOTIVE APPLICATIONS, IEEE Transactions on Device and Materials Reliability, vol. 3, no. 4, pages 191-196, Dec. 2003.

[Clabes04] Joachim Clabes, Joshua Friedrich, Mark Sweet, Jack DiLullo, Sam Chu, Donald Plass, James Dawson, Paul Muench, Larry Powell, and Michael Floyd. Design AND IMPLEMENTATION OF THE POWER5 ${ }^{\mathrm{TM}}$ MICROPROCESSOR, in 41 st annual Design Automation Conference, pages 670-672, 2004. 


\section{References}

[Coskun09] AK Coskun, JL Ayala, D Atienza, TS Rosing, and Y Leblebici. DYNAMIC THERMAL MANAGEMENT IN 3D MULTICORE ARCHITECTURES, in Design, Automation Test in Europe, pages 1410-1415, 2009.

[Dabholkar98] V Dabholkar, S Chakravarty, I Pomeranz, and S Reddy. TECHNIQUES FOR MINIMIZING POWER DISSIPATION IN SCAN AND COMBINATIONAL CIRCUITS DURING TEST APPLICATION, IEEE Transactions on Computer-Aided Design of Integrated Circuits and Systems, vol. 17, no. 12, pages 1325-1333, Dec. 1998.

[Davis94] Brendan Davis. The ECONOMics of Automatic TeSting. McGraw- Hill, 1994.

[Deutsch11] Sergej Deutsch, Vivek Chickermane, Brion Keller, Subhasish Mukherjee, Mario Konijnenburg, Erik Jan Marinissen, and Sandeep K Goel. Automation OF 3DDFT INSERTION, in 20 $0^{\text {th }}$ Asian Test Symposium, pages 395-400, 2011.

[Deutsch12] Sergej Deutsch, Krishnendu Chakrabarty, Shreepad Panth, and Sung Kyu Lim. TSV STRess-AWARE ATPG FOR 3D stacked ICs, in $21^{\text {st }}$ Asian Test Symposium, pages 31-36, 2012.

[Engelke08] P Engelke, I Polian, M Renovell, S Kundu, B Seshadri, and B Becker. ON DETECTION OF RESISTIVE BRIDGING DEFECTS BY LOW-TEMPERATURE AND LOWvOltage testing, IEEE Transactions on Computer-Aided Design of Integrated Circuits and Systems, vol. 27, no. 2, pages 327-338, Feb. 2008.

[Falkenauer98] Emanuel Falkenauer. GENETIC ALGORITHMS AND GROUPING PROBLEMS. Chichester; New York: Wiley, 1998.

[Flores99] P Flores, J Costa, H Neto, J Monteiro, and J Marques-Silva. Assignment AND REORDERING OF INCOMPLETELY SPECIFIED PATTERN SEQUENCES TARGETING MINIMUM POWER DISSIPATION, in $12^{\text {th }}$ International Conference On VLSI Design, pages $37-41,1999$.

[Flottes15] Marie-Lise Flottes, Joao Azevedo, Giorgio Di Natale, and Bruno Rouzeyre. SESSION-LESS BASED THERMAL-AWARE 3D-SIC TEST SCHEDULING, in 20th European Test Symposium, Cluj-Napoca, Romania, 2015.

[Frank10] T Frank, Cedrick Chappaz, P Leduc, L Arnaud, S Moreau, A Thuaire, R ElFarhane, and L Anghel. RELIABILITY APPROACH OF HIGH DENSITY THROUGH SiLICON VIA (TSV), in 12th Electronics Packaging Technology Conference, pages 321-324, 2010.

[Ganapathy10] Shrikanth Ganapathy, Ramon Canal, Antonio Gonzalez, and Antonio Rubio. Circuit PROPAGATION DELAY ESTIMATION THROUgh MULTIVARIATE REGRESSION-BASED MODELING UNDER SPATIO-TEMPORAL VARIABILITY, in Design, Automation \& Test in Europe, pages 417-422, 2010.

[Girard97] P Girard, C Landrault, S Pravossoudovitch, and D Severac. REDUCTION OF POWER CONSUMPTION DURING TEST APPLICATION BY TEST VECTOR ORDERING [VLSI CIRCUITS], Electronics Letters, vol. 33, no. 21, pages 1752-1754, Oct. 1997.

[GopiReddy14] L GopiReddy, LM Tolbert, B Ozpineci, and JOP Pinto. RAINFLOW ALGORITHM BASED LIFETIME ESTIMATION OF POWER SEMICONDUCTORS IN UTILITY APPliCATIONS, in $29^{\text {th }}$ annual Applied Power Electronics Conference and Exposition, pages 2293-2299, 2014.

[Gorev13] M Gorev, R Ubar, P Ellervee, S Devadze, J Raik, and M Min. AT-SPEED SELFTESTING OF HIGH-PERFORMANCE PIPE-LINED PROCESSING ARCHITECTURES, in NORCHIP Conference, pages 1-6, 2013. 


\section{References}

[Groebel01] DJ Groebel, A Mettas, and Feng-Bin Sun. DETERMINATION AND INTERPRETATION OF ACTIVATION ENERGY USING ACCELERATED-TEST DATA, in annual Reliability and Maintainability Symposium, pages 58-63, 2001.

[Hagihara97] Y Hagihara, S Inui, F Okamoto, M Nishida, T Nakamura, and H Yamada. FLOATING-POINT DATAPATHS WITH ONLINE BUILT-IN SELF SPEED TEST, IEEE Journal of Solid-State Circuits, vol. 32, no. 3, pages 444-449, Mar. 1997.

[Held97] M Held, P Jacob, G Nicoletti, P Scacco, and MH Poech. FAST POWER CYCLING TEST OF IGBT MODULES IN TRACTION APPLICATION, in International Conference on Power Electronics and Drive Systems, vol. 1, pages 425-430 vol.1, 1997.

[He06a] Zhiyuan He, Zebo Peng, and P Eles. Power CONSTRAined And DefeCtPROBABILITY DRIVEN SOC TEST SCHEDULING WITH TEST SET PARTITIONING, in Design, Automation and Test in Europe, vol. 1, pages 1-6, 2006.

[He06b] Zhiyuan He, Zebo Peng, P Eles, P Rosinger, and BM Al-Hashimi. TherMALAWARE SOC TEST SCHEDULING WITH TEST SET PARTITIONING AND INTERLEAVING, in 21st International Symposium on Defect and Fault Tolerance in VLSI Systems, pages $477-485,2006$.

[He07] Zhiyuan He, Zebo Peng, and P Eles. A HeUristic FOR THERMAL-SAFE SoC TEST SCHEDULING, in International Test Conference, pages 1-10, 2007.

[He08a] Zhiyuan He, Zebo Peng, and Petru Eles. Simulation-Driven Thermal-SAFE TEST TIME MINIMIZATION FOR SySTEM-ON-CHIP, in 17th Asian Test Symposium, pages 283-288, 2008.

[He08b] Zhiyuan He, Zebo Peng, Petru Eles, Paul Rosinger, and Bashir M Al-Hashimi. THERMAL-AWARE SOC TEST SCHEDULING WITH TEST SET PARTITIONING AND INTERLEAVING, Journal of Electronic Testing, vol. 24, no. 1-3, pages 247-257, Jan. 2008.

[He09] Zhiyuan He, Zebo Peng, and P Eles. THERMAL-AWARE TEST SCHEDULING FOR CORE-BASED SOC IN AN ABORT-ON-FIRST-FAIL TEST ENVIRONMENT, in 12th Euromicro Conference on Digital System Design, Architectures, Methods and Tools, pages 239-246, 2009.

[He10] Zhiyuan He, Zebo Peng, and P Eles. Multi-TemPerature testing For COREBASED SYSTEM-ON-CHIP, in Design, Automation Test in Europe, pages 208-213, 2010 .

[Higami13] Yoshinobu Higami, Hiroshi Takahashi, Shin-ya Kobayashi, and Kewal K Saluja. TEST GENERATION FOR DELAY FAULTS ON CLOCK LINES UNDER LAUNCH-ONCAPTURE TEST ENVIRONMENT, IEICE Transactions on Information and Systems, vol. E96-D, no. 6, pages 1323-1331, Jun. 2013.

[Higham05] N Higham. THE SCALING AND SQUARING METHOD FOR THE MATRIX EXPONENTIAL REVISITED, SIAM Journal on Matrix Analysis and Applications, vol. 26, no. 4, pages 1179-1193, Jan. 2005.

[Hirschmann06] D Hirschmann, D Tissen, S Schroder, and RW De Doncker. RELIABILITY PREDICTION FOR INVERTERS IN HYBRID ELECTRICAL VEHICLES, in 37th Power Electronics Specialists Conference, pages 1-6, 2006.

[Hirschmann07] D Hirschmann, D Tissen, S Schroder, and RW De Doncker. RELIABILITY PREDICTION FOR INVERTERS IN HYBRID ELECTRICAL VEHICLES, IEEE Transactions on Power Electronics, vol. 22, no. 6, pages 2511-2517, Nov. 2007. 


\section{References}

[Huang01] Yu Huang, Wu-Tung Cheng, Chien-Chung Tsai, N Mukherjee, O Samman, Y Zaidan, and SM Reddy. RESOURCE ALLOCATION AND TEST SCHEDULING FOR CONCURRENT TEST OF CORE-BASED SoC DESIGN, in 10th Asian Test Symposium, pages 265-270, 2001.

[Huang02] Yu Huang, SM Reddy, Wu-Tung Cheng, P Reuter, N Mukherjee, Chien-Chung Tsai, O Samman, and Y Zaidan. OPTIMAL CORE WRAPPER WIDTH SELECTION AND SOC TEST SCHEDULING BASED ON 3-D BIN PACKING ALGORITHM, in International Test Conference, pages 74-82, 2002.

[Huang06] Wei Huang, S Ghosh, S Velusamy, K Sankaranarayanan, K Skadron, and MR Stan. HOTSPOT: A COMPACT THERMAL MODELING METHODOLOGY FOR EARLY-STAGE VLSI DESIGN, IEEE Transactions on Very Large Scale Integration (VLSI) Systems, vol. 14, no. 5, pages 501-513, May. 2006.

[Huang07] Wei Huang. HotSPOT-A CHIP AND PACKAGE COMPACT THERMAL MODELING METHODOLOGY FOR VLSI DESIGN, Dissertation, University of Virginia, 2007.

[Ieee14a] IEEE P1838 3D-TEST WORKING Group, 2014. [Online]. Available: http://grouper.ieee.org/groups/3Dtest/. [Accessed: 29-May-2015].

[Ieee14b] IEEE STANDARD FOR ACCESS AND CONTROL OF INSTRUMENTATION EMBEDDED WITHIN A SEMICONDUCTOR DEVICE, IEEE Std 1687-2014, pages 1-283, Dec. 2014.

[Intel13] INTEL XEON E5-2600 v3 PROCESSOR OVERVIEW: HASWELL-EP UP TO 18 CORES, PC PERSPECTIVE, 2013. [Online]. Available: http://www.pcper.com/reviews/Processors/Intel-Xeon-E5-2600-v3-ProcessorOverview-Haswell-EP-18-Cores. [Accessed: 28-May-2015].

[Iyengar01] Vikram Iyengar and Krishnendu Chakrabarty. PRECEDENCE-BASED, PREEMPTIVE, AND POWER-CONSTRAINED TEST SCHEDULING FOR SYSTEM-ON-A-CHIP, in 19th VLSI Test Symposium, pages 368-374, 2001.

[Iyengar02] Vikram Iyengar, Krishnendu Chakrabarty, and Erik Jan Marinissen. ON USING RECTANGLE PACKING FOR SOC WRAPPER/TAM CO-OPTIMIZATION, in 20th VLSI Test Symposium, pages 253-258, 2002.

[Jagan10] L Jagan, C Hora, B Kruseman, S Eichenberger, AK Majhi, and V Kamakoti. IMPACT OF TEMPERATURE ON TEST QUALITY, in 23rd International Conference on VLSI Design, pages 276-281, 2010.

[Jedec09] TEMPERATURE CYCLING. Jedec solid state technology association, 2009.

[Jedec10] FAILURE MECHANISMS AND MODELS FOR SEMICONDUCTOR DEVICES, 2010. [Online]. Available: http://www.jedec.org/standards-documents/docs/jep-122e. [Accessed: 23-May-2014].

[Jiang14] T Jiang, C Wu, N Tamura, M Kunz, B Kim, H Son, M Suh, J Im, R Huang, and P Ho. STUDY OF STRESSES AND PLASTICITY IN THROUGH-SILICON VIA STRUCTURES FOR 3D INTERCONNECTS BY X-RAY MICRO-BEAM DIFFRACTION, IEEE Transactions on Device and Materials Reliability, vol. 14, no. 2, pages 698-703, June 2014.

[Kamto09] A Kamto, Y Liu, L Schaper, and SL Burkett. RELIABILITY STUDY OF THROUGHSILICON VIA (TSV) COPPER FILLED INTERCONNECTS, Thin Solid Films, vol. 518, no. 5, pages 1614-1619, Dec. 2009. 


\section{References}

[Kim10] Tak-Yung Kim and Taewhan Kim. ClOCK TREE SYNTHESIS WITH PRE-BOND TESTABILITY FOR 3D STACKED IC DESIGNS, in 47th Design Automation Conference, pages 723-728, 2010.

[Ko08] HF Ko and N Nicolici. AUTOMATED SCAN CHAIN DIVISION FOR REDUCING SHIFT AND CAPTURE POWER DURING BROADSIDE AT-SPEED TEST, IEEE Transactions on Computer-Aided Design of Integrated Circuits and Systems, vol. 27, no. 11, pages 2092-2097, Nov. 2008.

[Kumar12] P Kumar, I Dutta, and MS Bakir. INTERFACIAL EFFECTS DURING THERMAL CyCling of CU-FILled Through-Silicon Vias (TSV), Journal of Electronic Materials, vol. 41, no. 2, pages 322-335, Feb. 2012.

[Kundu05] S Kundu, P Engelke, I Polian, and B Becker. On DETECTION OF Resistive BRIDGING DEFECTS BY LOW-TEMPERATURE AND LOW-VOLTAGE TESTING, in 14 th Asian Test Symposium, pages 266-271, 2005.

[Kuo11] Chi-Wei Kuo and Hung-Yin Tsai. THERMAL STRESS ANALYSIS AND FAILURE MECHANISMS FOR THROUGH SILICON VIA ARRAY, in 6th International Microsystems, Packaging, Assembly and Circuits Technology Conference, pages 169-172, 2011.

[Kuo12] Chi-Wei Kuo and Hung-Yin Tsai. Thermal STRESS ANALYsis AND FaILURE MECHANISMS FOR THROUGH SILICON VIA ARRAY, in 13th Intersociety Conference on Thermal and Thermomechanical Phenomena in Electronic Systems, pages 202 206, 2012.

[Liao05] Weiping Liao, Lei He, and KM Lepak. TemPerature And Supply voltage AWARE PERFORMANCE AND POWER MODELING AT MICROARCHITECTURE LEVEL, IEEE Transactions on Computer-Aided Design of Integrated Circuits and Systems, vol. 24, no. 7, pages 1042-1053, Jul. 2005.

[Lin84] Tzu-Mu Lin and CA Mead. Signal DElay IN GENERAL RC NETwOrks, IEEE Transactions on Computer-Aided Design of Integrated Circuits and Systems, vol. 3, no. 4, pages 331-349, Oct. 1984.

[Li01] JC Li, Chao-Wen Tseng, and EJ McCluskey. TeSTING FOR RESISTIVE OPENS AND STUCK OPENS, in International Test Conference, pages 1049-1058, 2001.

[Liu04] Michael Liu, Wei-Shen Wang, and Michael Orshansky. LEAKAGE POWER REDUCTION BY DUAL-VTH DESIGNS UNDER PROBABILISTIC ANALYSIS OF VTH VARIATION, in International Symposium on Low Power Electronics and Design, pages 2-7, 2004.

[Loi08] Igor Loi, Subhasish Mitra, Thomas H Lee, Shinobu Fujita, and Luca Benini. A LOW-OVERHEAD FAULT TOLERANCE SCHEME FOR TSV-BASED 3D NETWORK ON CHIP LINKS, in IEEE/ACM International Conference on Computer-Aided Design, pages 598-602, 2008.

[Long04] E Long, WR Daasch, R Madge, and B Benware. Detection of TEMPERAture SENSITIVE DEFECTS USING ZTC, in 22nd VLSI Test Symposium, 2004. Proceedings, pages $185-190,2004$.

[Lu07] Hua Lu, T Tilford, and DR Newcombe. LIFETIME PREDICTION FOR POWER ELECTRONICS MODULE SUBSTRATE MOUNT-DOWN SOLDER INTERCONNECT, in International Symposium on High Density packaging and Microsystem Integration, pages $1-10,2007$. 


\section{References}

[Manikandan11] P Manikandan, BB Larsen, and EJ Aas. AN ENHANCED PATH DELAY FAULT SIMULATOR FOR COMBINATIONAL CIRCUITS, in 14th Euromicro Conference on Digital System Design, pages 375-381, 2011.

[Marinissen00] EJ Marinissen, SK Goel, and M Lousberg. WRAPPER DESIGN FOR EMBEDDED CORE TEST, in International Test Conference, pages 911-920, 2000.

[Marinissen02] EJ Marinissen, V Iyengar, and K Chakrabarty. A SET OF BENCHMARKS FOR MODUlAR TESTING OF SoCs, in International Test Conference, pages 519-528, 2002.

[Marinissen09] Erik Jan Marinissen and Yervant Zorian. TEsting 3D ChIPS CONTAINING THROUGH-SILICON VIAS, in International Test Conference, pages 1-11, 2009.

[Marinissen10a] Erik Jan Marinissen, Chun-Chuan Chi, Jouke Verbree, and Mario Konijnenburg. 3D DFT ARCHITECTURE FOR PRE-BOND AND POST-BOND TESTING, in International 3D Systems Integration Conference, pages 1-8, 2010.

[Marinissen10b] Erik Jan Marinissen, Jouke Verbree, and Mario Konijnenburg. A STRUCTURED AND SCALABLE TEST ACCESS ARCHITECTURE FOR TSV-BASED 3D STACKED ICS, in 28th VLSI Test Symposium, pages 269-274, 2010.

[Marinissen10c] Erik Jan Marinissen. ChALlEnges IN TESTING TSV-BASED 3D sTACKED ICs: TEST FLOWS, TEST CONTENTS, AND TEST ACCESS, in Asia Pacific Conference on Circuits and Systems, pages 544-547, 2010.

[Marinissen12a] Erik Jan Marinissen. Challenges And EMERGING SOlutions IN TESTING TSVBASED 2 1/2D-AND 3D-STACKED ICS, in Design, Automation and Test in Europe, pages $1277-1282,2012$.

[Marinissen12b] Erik Jan Marinissen, Chun-Chuan Chi, Mario Konijnenburg, and Jouke Verbree. A DFT ARCHITECTURE FOR 3D-SICS BASED ON A STANDARDIZABLE DIE WRAPPER, Journal of Electronic Testing, vol. 28, no. 1, pages 73-92, Feb. 2012.

[Matsuishi68] M Matsuishi and T Endo. Fatigue of METALs SubJeCted to VARYing STRESS, Japan Society of Mechanical Engineers, Fukuoka, Japan, pages 37-40, 1968.

[Maulik00] Ujjwal Maulik and Sanghamitra Bandyopadhyay. GENETIC ALGORITHM-BASED CLUSTERING TECHNIQUe, Pattern recognition, vol. 33, no. 9, pages 1455-1465, 2000 .

[Mi104] TEMPERATURE CYCLING (MIL-STD-883; METHOD 1010), DLA Land and Maritime Mil. Specs \& Drawings, Jun-2004. [Online]. Available: http://www.landandmaritime.dla.mil/programs/milspec/ListDocs.aspx?BasicDoc= MIL-STD-883. [Accessed: 28-May-2014].

[Miller01] Mark Miller. NEXT GENERATION BURN-IN AND TEST SYSTEMS FOR ATHLON MICROPROCESSORS: HYBRID BURN-IN, in BiTS Workshop, 2001.

[Millican14] SK Millican and KK Saluja. A TEST PARTITIONING TECHNIQUE FOR SCHEDULING TESTS FOR THERMALLY CONSTRAINED 3D INTEGRATED CIRCUITS, in 27th International Conference on VLSI Design, pages 20-25, 2014.

[Mohapatra07] Debabrata Mohapatra, Georgios Karakonstantis, and Kaushik Roy. Low-POWER PROCESS-VARIATION TOLERANT ARITHMETIC UNITS USING INPUT-BASED ELASTIC CLOCKING, in International Symposium on Low Power Electronics and Design, pages 74-79, 2007. 


\section{References}

[Monda107] Mosin Mondal, Andrew J Ricketts, Sami Kirolos, Tamer Ragheb, Greg Link, Narayanan Vijaykrishnan, and Yehia Massoud. THERMALLY ROBUST CLOCKING SCHEMES FOR 3D INTEGRATED CIRCUITS, in Design, Automation \& Test in Europe, pages $1-6,2007$.

[Murray12] Conal E Murray, ET Ryan, Paul R Besser, C Witt, Jean L Jordan-Sweet, and MF Toney. EVOLUTION OF STRESS GRADIENTS IN CU FILMS AND FEATURES INDUCED BY CAPPING LAYERS, Microelectronic Engineering, vol. 92, pages 95-100, Apr. 2012.

[Musallam12] M Musallam and CM Johnson. AN EFFICIENT IMPLEMENTATION OF THE RAINFLOW COUNTING ALGORITHM FOR LIFE CONSUMPTION ESTIMATION, IEEE Transactions on Reliability, vol. 61, no. 4, pages 978-986, Dec. 2012.

[Nebe197] Wolfgang Nebel and Jean P Mermet. LOW POWER DESIGN IN DEEP SUBMICRON ELECTRONICS. Norwell, MA, USA: Kluwer Academic Publishers, 1997.

[Needham98] Wayne Needham, Cheryl Prunty, and Eng Hong Yeoh. High volumE MICROPROCESSOR TEST ESCAPES, AN ANALYSIS OF DEFECTS OUR TESTS ARE MISSING, in International Test Conference, pages 25-34, 1998.

[Nigh98] P Nigh, D Vallett, P Patel, J Wright, F Motika, D Forlenza, R Kurtulik, and W Chong. FAILURE ANALYSIS OF TIMING AND IDDQ-ONLY FAILURES FROM THE SEMATECH TEST METHODS EXPERIMENT, in International Test Conference, pages 43-52, 1998.

[Noia10a] Brandon Noia, Krishnendu Chakrabarty, and Erik Jan Marinissen. OptiMIZATION METHODS FOR POST-BOND DIE-INTERNAL/EXTERNAL TESTING IN 3D STACKED ICS, in International Test Conference, pages 1-9, 2010.

[Noia10b] Brandon Noia, Sandeep Kumar Goel, Krishnendu Chakrabarty, Erik Jan Marinissen, and Jouke Verbree. TEST-ARCHITECTURE OPTIMIZATION FOR TSVBASED 3D STACKED ICs, in 15th European Test Symposium, pages 24-29, 2010.

[Noia11] Brandon Noia and Krishnendu Chakrabarty. TESTING AND DESIGN-FORTESTABILITY TECHNIQUES FOR 3D INTEGRATED CIRCUITS, $20^{\text {th }}$ Asian Test Symposium, pages 474-479, 2011.

[Noia12] Brandon Noia, Krishnendu Chakrabarty, and Erik Jan Marinissen. OptIMIZATION METHODS FOR POST-BOND TESTING OF 3D STACKED ICS, Journal of Electronic Testing, vol. 28, no. 1, pages 103-120, Feb. 2012.

[Nowka08] Kevin Nowka. SuRVIVAL OF VLSI DESIGN - COPING WITH DEVICE VARIABILITY AND UnCERTAINTY, in Circuits and Systems Workshop: System-on-Chip - Design, Applications, Integration, and Software, Dallas, pages 1-6, 2008.

[Nvidia12] NVIDIA's NEXT GENERATION CUDA COMPUTE ARCHITECTURE: KEPLER GK110. 2012 .

[Oberg03] Johnny Oberg. NeTwORKS ON CHIP, A. Jantsch and H. Tenhunen, Eds. Hingham, MA, USA: Kluwer Academic Publishers, pages 153-172, 2003.

[Okoro12] C Okoro and YS Obeng. EFFECT OF THERMAL CYCLING ON THE SIGNAL INTEGRITY AND MORPHOLOGY OF TSV ISOLATION LINER- SIO2, in International Interconnect Technology Conference, pages 1-3, 2012.

[Okoro14] Chukwudi Okoro, June W Lau, Fardad Golshany, Klaus Hummler, and Yaw S Obeng. A DETAILED FAILURE ANALYSIS EXAMINATION OF THE EFFECT OF THERMAL 


\section{References}

CYCLING ON CU TSV RELIABILITY, IEEE Transactions on Electron Devices, vol. 61, no. 1, pages 15-22, Jan. 2014.

[Oppenheim97] Alan V Oppenheim, Alan S Willsky, and Syed Hamid Nawab. SigNALS AND SYSTEMS, $2^{\text {nd }}$ ed. Upper Saddle River, N.J: Prentice Hall, 1997.

[Pak11] JS Pak, Mohit Pathak, Sung Kyu Lim, and David Z Pan. Modeling OF ELECTROMIGRATION IN THROUGH-SILICON-VIA BASED 3D IC, in 61st Electronic Components and Technology Conference, pages 1420-1427, 2011.

[Patil07] Srinivas Patil. AT-SPEed SCAN TESTS: Reality or Fantasy? Panel 1.4, in International Test Conference, pages 1-1, 2007.

[Plas10] G Van der Plas, S Thijs, D Linten, G Katti, P Limaye, A Mercha, M Stucchi, H Oprins, B Vandevelde, N Minas, M Cupac, M Dehan, M Nelis, R Agarwal, W Dehaene, Y Travaly, E Beyne, and $\mathrm{P}$ Marchal. VERIFYING ELECTRICAL/THERMAL/THERMO-MECHANICAL BEHAVIOR OF A 3D STACK Challenges AND solutions, in Custom Integrated Circuits Conference, pages $1-4,2010$.

[Plas11] Geert Van Der Plas, Erik-Jan Marinissen, Nikolaos Minas, and Paul Marchal. METHOD AND DEVICE FOR TESTING TSVS IN A 3D CHIP STACK, U.S. Patent US20110102011 A105-May-2011.

[Poli07] Riccardo Poli, James Kennedy, and Tim Blackwell. PARTICLE SWARM OPTIMIZATION, Swarm Intelligence, vol. 1, no. 1, pages 33-57, Jun. 2007.

[Press07] William H Press. NuMERICAL RECIPES: THE ART OF SCIENTIFIC COMPUTING, 3rd ed. Cambridge, UK; New York: Cambridge University Press, 2007.

[Raina07] Rajesh Raina. AT-SPEED SCAN TESTS: REALITY OR FANTASY? PANEL 1.5, in International Test Conference, pages 1-2, 2007.

[Rao03] Rajeev Rao, Ashish Srivastava, David Blaauw, and Dennis Sylvester. STATISTICAL ESTIMATION OF LEAKAGE CURRENT CONSIDERING INTER-AND INTRA-DIE PROCESS VARIATION, in International Symposium on Low Power Electronics and Design, pages $84-89,2003$.

[Rohani13] Alireza Rohani and Hans G Kerkhoff. RAPID TRANSIENT FAULT INSERTION IN LARGE DIGITAL SYSTEMS, Microprocessors and Microsystems, vol. 37, no. 2, pages 147-154, Mar. 2013.

[Rosinger02] PM Rosinger, BM Al-Hashimi, and N Nicolici. POWER PROFILE MANIPULATION: A NEW APPROACH FOR REDUCING TEST APPLICATION TIME UNDER POWER CONSTRAINTS, IEEE Transactions on Computer-Aided Design of Integrated Circuits and Systems, vol. 21, no. 10, pages 1217-1225, Oct. 2002.

[Rosinger06] Paul Rosinger, Bashir M Al-Hashimi, and Krishnendu Chakrabarty. THERMALSAFE TEST SCHEDULING FOR CORE-BASED SYSTEM-ON-CHIP INTEGRATED CIRCUITS, IEEE Transactions on Computer-Aided Design of Integrated Circuits and Systems, vol. 25, no. 11, pages 2502-2512, 2006.

[Samii06] Soheil Samii, Erik Larsson, Krishnendu Chakrabarty, and Zebo Peng. CYCLEACCURATE TEST POWER MODELING AND ITS APPLICATION TO SOC TEST SCHEDULING, in International Test Conference, pages 1-10, 2006.

[Santarini14] Mike Santarini. XILINX ShIPS INDUSTRY'S FIRST 20-NM ALL PROGRAMMABLE DEVICES, Xcell, vol. 1, no. 86, page 14, 2014. 


\section{References}

[Sarangi08] SR Sarangi, B Greskamp, R Teodorescu, J Nakano, A Tiwari, and J Torrellas. VARIUS: A MODEL OF PROCESS VARIATION AND RESULTING TIMING ERRORS FOR MICROARCHITECTS, IEEE Transactions on Semiconductor Manufacturing, vol. 21, no. 1, pages 3-13, Feb. 2008.

[Schuermyer04] C Schuermyer, J Ruffler, R Daasch, and R Madge. Minimum TESTING REQUIREMENTS TO SCREEN TEMPERATURE DEPENDENT DEFECTS, in International Test Conference, pages 300-308, 2004.

[Segura02] J Segura, A Keshavarzi, J Soden, and C Hawkins. PARAMETRIC FAILuRES IN CMOS ICS - A DEFECT-BASED ANALYSIS, in International Test Conference, pages 90-99, 2002.

[Segura04] Jaume Segura and Charles F Hawkins. CMOS ELECTRONICS: HOW IT WORKS, HOW IT FAILS. Hoboken, NJ, USA: John Wiley \& Sons, Inc., 2004.

[Semenov03] O Semenov, A Vassighi, M Sachdev, A Keshavarzi, and CF Hawkins. EFFECT OF CMOS TECHNOLOGY SCALING ON THERMAL MANAGEMENT DURING BURN-IN, IEEE Transactions on Semiconductor Manufacturing, vol. 16, no. 4, pages 686-695, Nov. 2003.

[SenGupta12] Breeta SenGupta, Urban Ingelsson, and Erik Larsson. ScHEDULING TESTS FOR 3D STACKED CHIPS UNDER POWER CONSTRAINTS, Journal of Electronic Testing, vol. 28, no. 1, pages 121-135, Feb. 2012.

[Shibin15] Konstantin Shibin, Vivek Chickermane, Brion Keller, Christos Papameletis, and Erik Jan Marinissen. AT-SPEED DELAY TESTING OF INTER-DIE CONNECTIONS OF 2.5D- AND 3D-SICs, in 20th European Test Symposium, 2015.

[Smorodin08] T Smorodin, J Wilde, P Alpern, and M Stecher. A TEMPERATURE-GRADIENTINDUCED FAILURE MECHANISM IN METALLIZATION UNDER FAST THERMAL CYCLING, IEEE Transactions on Device and Materials Reliability, vol. 8, no. 3, pages 590-599, Sep. 2008.

[Srivastava02] Ashish Srivastava, Robert Bai, David Blaauw, and Dennis Sylvester. MoDELING AND ANALYSIS OF LEAKAGE POWER CONSIDERING WITHIN-DIE PROCESS VARIATIONS, in International Symposium on Low Power Electronics and Design, pages 64-67, 2002.

[Srivastava04] Ashish Srivastava, Dennis Sylvester, and David Blaauw. STATISTICAL OPTIMIZATION OF LEAKAGE POWER CONSIDERING PROCESS VARIATIONS USING DUAL-VTH AND SIZING, in $41^{\text {st }}$ annual Design Automation Conference, pages 773 $778,2004$.

[Stan03] Mircea R Stan, Kevin Skadron, Marco Barcella, Wei Huang, Karthik Sankaranarayanan, and Sivakumar Velusamy. HoTSPOT: A DYNAMIC COMPACT THERMAL MODEL AT THE PROCESSOR-ARCHITECTURE LEVEL, Microelectronics Journal, vol. 34, no. 12, pages 1153-1165, 2003.

[Syed10] A Syed. LIMITATIONS OF NORRIS-LANDZBERG EQUATION AND APPLICATION OF DAMAGE ACCUMULATION BASED METHODOLOGY FOR ESTIMATING ACCELERATION FACTORS FOR PB FREE SOLDERS, in 11th International Conference on Thermal, Mechanical Multi-Physics Simulation, and Experiments in Microelectronics and Microsystems, pages 1-11, 2010.

[Tadayon00] Pooya Tadayon. ThERMAL CHALLENGES DURING MICROPROCESSOR TESTING, Intel Technology Journal, vol. 4, no. 3, pages 1-8, 2000. 


\section{References}

[Taouil10a] Mottaqiallah Taouil, Said Hamdioui, Kees Beenakker, and Erik Jan Marinissen. Test COST ANALYSIS FOR 3D DIE-TO-WAFER STACKING, in $19^{\text {th }}$ Asian Test Symposium, pages 435-441, 2010.

[Taouil10b] Mottaqiallah Taouil, Said Hamdioui, Jouke Verbree, and Erik Jan Marinissen. ON MAXIMIZING THE COMPOUND YIELD FOR 3D WAFER-TO-WAFER STACKED ICS, in International Test Conference, pages 1-10, 2010.

[Taouil11] Mottaqiallah Taouil, Said Hamdioui, and Erik Jan Marinissen. How SIGNIFICANT WILL BE THE TEST COST SHARE FOR 3D DIE-TO-WAFER STACKED-ICs?, in 6th International Conference on Design \& Technology of Integrated Systems in Nanoscale Era, pages 1-6, 2011.

[Taouil12] Mottaqiallah Taouil, Said Hamdioui, Kees Beenakker, and Erik Jan Marinissen. TEST IMPACT ON THE OVERALL DIE-TO-WAFER 3D STACKED IC COST, Journal of Electronic Testing, vol. 28, no. 1, pages 15-25, Feb. 2012.

[Tseng00] Chao-Wen Tseng, EJ McCluskey, Xiaoping Shao, and DM Wu. Cold DELAY DEFECT SCREENING, in 18th VLSI Test Symposium, pages 183-188, 2000.

[Tudu09] JT Tudu, E Larsson, V Singh, and VD Agrawal. ON MINIMIZATION OF PEAK POWER FOR SCAN CIRCUIT DURING TEST, in 14th European Test Symposium, pages 25-30, 2009.

[Ukhov12] Ivan Ukhov, Min Bao, Petru Eles, and Zebo Peng. SteADY-STATE DynAmiC TEMPERATURE ANALYSIS AND RELIABILITY OPTIMIZATION FOR EMBEDDED MULTIPROCESSOR SYSTEMS, in 49th annual Design Automation Conference, New York, NY, USA, pages 197-204, 2012.

[Ukhov14a] I Ukhov, $\mathrm{P}$ Eles, and $\mathrm{Z}$ Peng. Probabilistic ANAlysis OF POWER AND TEMPERATURE UNDER PROCESS VARIATION FOR ELECTRONIC SYSTEM DESIGN, IEEE Transactions on Computer-Aided Design of Integrated Circuits and Systems, vol. 33, no. 6, pages 931-944, Jun. 2014.

[Ukhov14b] I Ukhov, P Eles, and Z Peng. TEMPERATURE-CENTRIC RELIABILITY ANALYSIS AND OPTIMIZATION OF ELECTRONIC SYSTEMS UNDER PROCESS VARIATION, IEEE Transactions on Very Large Scale Integration (VLSI) Systems, vol. PP, no. 99, pages 1-1, 2014.

[Vassighi06] Arman Vassighi and Manoj Sachdev. THERMAL AND POWER MANAGEMENT OF INTEGRATED CIRCUITS. Boston: Kluwer Academic Publishers, 2006.

[Vasudevan08] V Vasudevan and Xuejun Fan. AN ACCELERATION MODEL FOR LEAD-FREE (SAC) SOLDER JOINT RELIABILITY UNDER THERMAL CYCLING, in 58th Electronic Components and Technology Conference, pages 139-145, 2008.

[Velenis10] Dimitrios Velenis, Erik Jan Marinissen, and Eric Beyne. Cost EFFECTIVENESS OF 3D InTEGRATION OPTIONS, in International 3D Systems Integration Conference, pages $1-6,2010$.

[Verbree10] Jouke Verbree, Erik Jan Marinissen, Philippe Roussel, and Dimitrios Velenis. ON THE COST-EFFECTIVENESS OF MATCHING REPOSITORIES OF PRE-TESTED WAFERS FOR WAFER-TO-WAFER 3D CHIP STACKING, in 15th European Test Symposium, pages 36-41, 2010.

[Vinay10] NS Vinay, Indira Rawat, Erik Larsson, MS Gaur, and Virendra Singh. THERMAL AWARE TEST SCHEDULING FOR STACKED MULTI-CHIP-MODULES, in East-West Design \& Test Symposium, pages 343-349, 2010. 


\section{References}

[Wen11] Xiaoqing Wen, Kazunari Enokimoto, Kohei Miyase, Yuta Yamato, Michael A Kochte, Seiji Kajihara, Patrick Girard, and Mohammad Tehranipoor. PowERAWARE TEST GENERATION WITH GUARANTEED LAUNCH SAFETY FOR AT-SPEED SCAN TESTING, in 29th VLSI Test Symposium, pages 166-171, 2011.

[Wu10] Sean $\mathrm{H}$ Wu, Alexander Tetelbaum, and Li-C Wang. How DOES INVERSE TEMPERATURE DEPENDENCE AFFECT TIMING SIGN-OFF, in Emerging Technologies and Circuits, A. Amara, T. Ea, and M. Belleville, Eds. Springer Netherlands, pages 179-189, 2010.

[Yao09] Chunhua Yao, Kewal K Saluja, and Parameswaran Ramanathan. PARTITION BASED SOC TEST SCHEDULING WITH THERMAL AND POWER CONSTRAINTS UNDER DEEP SUBMICRON TECHNOLOGIES, in Asian Test Symposium, pages 281-286, 2009.

[Yao11a] Chunhua Yao, Kewal K Saluja, and Parameswaran Ramanathan. Power AND THERMAL CONSTRAINED TEST SCHEDULING UNDER DEEP SUBMICRON TECHNOLOGIES, IEEE Transactions on Computer-Aided Design of Integrated Circuits and Systems, vol. 30, no. 2, pages 317-322, Feb. 2011.

[Yao11b] C Yao, KK Saluja, and P Ramanathan. TEMPERATURE DEPENDENT TEST SCHEDULING FOR MULTI-CORE SYSTEM-ON-CHIP, in 20th Asian Test Symposium, pages 27-32, 2011.

[Yao11c] Chunhua Yao, Kewal K Saluja, and Parameswaran Ramanathan. THERMALAWARE TEST SCHEDULING USING ON-CHIP TEMPERATURE SENSORS, in 24th International Conference on VLSI Design, pages 376-381, 2011.

[Yu09] TE Yu, T Yoneda, K Chakrabarty, and H Fujiwara. TEST INFRASTRUCTURE DESIGN FOR CORE-BASED SYSTEM-ON-CHIP UNDER CYCLE-ACCURATE THERMAL CONSTRAINTS, in Asia and South Pacific Design Automation Conference, pages 793-798, 2009.

[Zhang13] Dingyou Zhang, K Hummler, L Smith, and JJQ Lu. BACKSIDE TSV PROTRUSION INDUCED BY THERMAL SHOCK AND THERMAL CYCLING, in 63rd Electronic Components and Technology Conference, pages 1407-1413, 2013.

[Zhao10] Wei Zhao, Junxia Ma, M Tehranipoor, and S Chakravarty. PoweR-SAFE APPLICATION OF TRANSITION DELAY FAULT PATTERNS CONSIDERING CURRENT LIMIT DURING WAFER TEST, in 19th Asian Test Symposium, pages 301-306, 2010.

[Zhuo10] Cheng Zhuo, Dennis Sylvester, and David Blaauw. Process VARIATION AND TEMPERAture-aware reliability management, in Design, Automation and Test in Europe, pages 580-585, 2010.

[Zorian93] Y Zorian. A DISTRIBUTED BIST CONTROL SCHEME FOR COMPLEX VLSI DEVICES, in $11^{\text {th }}$ annual VLSI Test Symposium, pages 4-9, 1993.

[Zou03] Wei Zou, SM Reddy, I Pomeranz, and Yu Huang. SoC TEST SCHEDULING USING SimUlated ANNEALING, in 21 $1^{\text {st }}$ VLSI Test Symposium, pages 325-330, 2003.

[Zschech02] Ehrenfried Zschech, Eckhard Langer, Hans-Juergen Engelmann, and Kornelia Dittmar. PHYSICAL FAILURE ANALYSIS IN SEMICONDUCTOR INDUSTRYCHALLENGES OF THE COPPER INTERCONNECT PROCESS, Materials Science in Semiconductor Processing, vol. 5, no. 4-5, pages 457-464, Aug. 2002. 



\section{Dissertations}

\section{Linköping Studies in Science and Technology Linköping Studies in Arts and Science Linköping Studies in Statistics Linköpings Studies in Information Science}

Linköping Studies in Science and Technology

No 14 Anders Haraldsson: A Program Manipulation System Based on Partial Evaluation, 1977, ISBN 917372-144-1.

No 17 Bengt Magnhagen: Probability Based Verification of Time Margins in Digital Designs, 1977, ISBN 91-7372157-3.

No 18 Mats Cedwall: Semantisk analys av processbeskrivningar i naturligt språk, 1977, ISBN 91- 7372168-9.

No 22 Jaak Urmi: A Machine Independent LISP Compiler and its Implications for Ideal Hardware, 1978, ISBN 91-7372-188-3.

No 33 Tore Risch: Compilation of Multiple File Queries in a Meta-Database System 1978, ISBN 91- 7372-232-4.

No 51 Erland Jungert: Synthesizing Database Structures from a User Oriented Data Model, 1980, ISBN 917372-387-8.

No 54 Sture Hägglund: Contributions to the Development of Methods and Tools for Interactive Design of Applications Software, 1980, ISBN 91-7372-404-1.

No 55 Pär Emanuelson: Performance Enhancement in a Well-Structured Pattern Matcher through Partial Evaluation, 1980, ISBN 91-7372-403-3.

No 58 Bengt Johnsson, Bertil Andersson: The HumanComputer Interface in Commercial Systems, 1981, ISBN 91-7372-414-9.

No 69 H. Jan Komorowski: A Specification of an Abstract Prolog Machine and its Application to Partial Evaluation, 1981, ISBN 91-7372-479-3.

No 71 René Reboh: Knowledge Engineering Techniques and Tools for Expert Systems, 1981, ISBN 91-7372489-0.

No 77 Östen Oskarsson: Mechanisms of Modifiability in large Software Systems, 1982, ISBN 91- 7372-527-7.

No 94 Hans Lunell: Code Generator Writing Systems, 1983, ISBN 91-7372-652-4.

No 97 Andrzej Lingas: Advances in Minimum Weight Triangulation, 1983, ISBN 91-7372-660-5.

No 109 Peter Fritzson: Towards a Distributed Programming Environment based on Incremental Compilation, 1984, ISBN 91-7372-801-2.

No 111 Erik Tengvald: The Design of Expert Planning Systems. An Experimental Operations Planning System for Turning, 1984, ISBN 91-7372- 805-5.

No 155 Christos Levcopoulos: Heuristics for Minimum Decompositions of Polygons, 1987, ISBN 91-7870133-3.

No 165 James W. Goodwin: A Theory and System for NonMonotonic Reasoning, 1987, ISBN 91-7870-183-X.
No 170 Zebo Peng: A Formal Methodology for Automated Synthesis of VLSI Systems, 1987, ISBN 91-7870-225-9.

No 174 Johan Fagerström: A Paradigm and System for Design of Distributed Systems, 1988, ISBN 91-7870301-8.

No 192 Dimiter Driankov: Towards a Many Valued Logic of Quantified Belief, 1988, ISBN 91-7870-374-3.

No 213 Lin Padgham: Non-Monotonic Inheritance for an Object Oriented Knowledge Base, 1989, ISBN 917870-485-5.

No 214 Tony Larsson: A Formal Hardware Description and Verification Method, 1989, ISBN 91-7870-517-7.

No 221 Michael Reinfrank: Fundamentals and Logical Foundations of Truth Maintenance, 1989, ISBN 917870-546-0.

No 239 Jonas Löwgren: Knowledge-Based Design Support and Discourse Management in User Interface Management Systems, 1991, ISBN 91-7870-720-X.

No 244 Henrik Eriksson: Meta-Tool Support for Knowledge Acquisition, 1991, ISBN 91-7870-746-3.

No 252 Peter Eklund: An Epistemic Approach to Interactive Design in Multiple Inheritance Hierarchies, 1991, ISBN 91-7870-784-6.

No 258 Patrick Doherty: NML3 - A Non-Monotonic Formalism with Explicit Defaults, 1991, ISBN 917870-816-8.

No 260 Nahid Shahmehri: Generalized Algorithmic Debugging, 1991, ISBN 91-7870-828-1.

No 264 Nils Dahlbäck: Representation of DiscourseCognitive and Computational Aspects, 1992, ISBN 91-7870-850-8.

No 265 Ulf Nilsson: Abstract Interpretations and Abstract Machines: Contributions to a Methodology for the Implementation of Logic Programs, 1992, ISBN 917870-858-3.

No 270 Ralph Rönnquist: Theory and Practice of Tensebound Object References, 1992, ISBN 91-7870-873-7.

No 273 Björn Fjellborg: Pipeline Extraction for VLSI Data Path Synthesis, 1992, ISBN 91-7870-880-X.

No 276 Staffan Bonnier: A Formal Basis for Horn Clause Logic with External Polymorphic Functions, 1992, ISBN 91-7870-896-6.

No 277 Kristian Sandahl: Developing Knowledge Management Systems with an Active Expert Methodology, 1992, ISBN 91-7870-897-4

No 281 Christer Bäckström: Computational Complexity of Reasoning about Plans, 1992, ISBN 91-7870-979-2.

No 292 Mats Wirén: Studies in Incremental Natural Language Analysis, 1992, ISBN 91-7871-027-8.

No 297 Mariam Kamkar: Interprocedural Dynamic Slicing with Applications to Debugging and Testing, 1993, ISBN 91-7871-065-0. 
No302 Tingting Zhang: A Study in Diagnosis Using Classification and Defaults, 1993, ISBN 91-7871-078-2

No312 Arne Jönsson: Dialogue Management for Natural Language Interfaces - An Empirical Approach, 1993, ISBN 91-7871-110-X.

No 338 Simin Nadjm-Tehrani: Reactive Systems in Physical Environments: Compositional Modelling and Framework for Verification, 1994, ISBN 91-7871-237-8.

No 371 Bengt Savén: Business Models for Decision Support and Learning. A Study of Discrete-Event Manufacturing Simulation at Asea/ABB 1968-1993, 1995, ISBN 91-7871-494-X.

No 375 Ulf Söderman: Conceptual Modelling of Mode Switching Physical Systems, 1995, ISBN 91-7871-5164.

No 383 Andreas Kågedal: Exploiting Groundness in Logic Programs, 1995, ISBN 91-7871-538-5.

No 396 George Fodor: Ontological Control, Description, Identification and Recovery from Problematic Control Situations, 1995, ISBN 91-7871-603-9.

No 413 Mikael Pettersson: Compiling Natural Semantics, 1995, ISBN 91-7871-641-1.

No 414 Xinli Gu: RT Level Testability Improvement by Testability Analysis and Transformations, 1996, ISBN 91-7871-654-3.

No 416 Hua Shu: Distributed Default Reasoning, 1996, ISBN 91-7871-665-9.

No 429 Jaime Villegas: Simulation Supported Industrial Training from an Organisational Learning Perspective - Development and Evaluation of the SSIT Method, 1996, ISBN 91-7871-700-0.

No 431 Peter Jonsson: Studies in Action Planning: Algorithms and Complexity, 1996, ISBN 91-7871-7043.

No 437 Johan Boye: Directional Types in Logic Programming, 1996, ISBN 91-7871-725-6.

No 439 Cecilia Sjöberg: Activities, Voices and Arenas: Participatory Design in Practice, 1996, ISBN 91-7871728-0.

No 448 Patrick Lambrix: Part-Whole Reasoning in Description Logics, 1996, ISBN 91-7871-820-1.

No 452 Kjell Orsborn: On Extensible and Object-Relational Database Technology for Finite Element Analysis Applications, 1996, ISBN 91-7871-827-9.

No 459 Olof Johansson: Development Environments for Complex Product Models, 1996, ISBN 91-7871-855-4.

No 461 Lena Strömbäck: User-Defined Constructions in Unification-Based Formalisms, 1997, ISBN 91-7871857-0.

No 462 Lars Degerstedt: Tabulation-based Logic Programming: A Multi-Level View of Query Answering, 1996, ISBN 91-7871-858-9.

No 475 Fredrik Nilsson: Strategi och ekonomisk styrning En studie av hur ekonomiska styrsystem utformas och används efter företagsförvärv, 1997, ISBN 917871-914-3.

No 480 Mikael Lindvall: An Empirical Study of Requirements-Driven Impact Analysis in Object-Oriented Software Evolution, 1997, ISBN 91-7871-927-5.
No 485 Göran Forslund: Opinion-Based Systems: The Cooperative Perspective on Knowledge-Based Decision Support, 1997, ISBN 91-7871-938-0.

No 494 Martin Sköld: Active Database Management Systems for Monitoring and Control, 1997, ISBN 917219-002-7.

No 495 Hans Olsén: Automatic Verification of Petri Nets in a CLP framework, 1997, ISBN 91-7219-011-6.

No 498 Thomas Drakengren: Algorithms and Complexity for Temporal and Spatial Formalisms, 1997, ISBN 917219-019-1.

No 502 Jakob Axelsson: Analysis and Synthesis of Heterogeneous Real-Time Systems, 1997, ISBN 91-7219-035-3.

No 503 Johan Ringström: Compiler Generation for DataParallel Programming Languages from Two-Level Semantics Specifications, 1997, ISBN 91-7219-045-0.

No 512 Anna Moberg: Närhet och distans - Studier av kommunikationsmönster i satellitkontor och flexibla kontor, 1997, ISBN 91-7219-119-8.

No 520 Mikael Ronström: Design and Modelling of a Parallel Data Server for Telecom Applications, 1998, ISBN 91-7219-169-4.

No 522 Niclas Ohlsson: Towards Effective Fault Prevention - An Empirical Study in Software Engineering, 1998, ISBN 91-7219-176-7.

No 526 Joachim Karlsson: A Systematic Approach for Prioritizing Software Requirements, 1998, ISBN 917219-184-8.

No 530 Henrik Nilsson: Declarative Debugging for Lazy Functional Languages, 1998, ISBN 91-7219-197-x.

No 555 Jonas Hallberg: Timing Issues in High-Level Synthesis, 1998, ISBN 91-7219-369-7.

No 561 Ling Lin: Management of 1-D Sequence Data - From Discrete to Continuous, 1999, ISBN 91-7219-402-2.

No 563 Eva L Ragnemalm: Student Modelling based on Collaborative Dialogue with a Learning Companion, 1999, ISBN 91-7219-412-X.

No 567 Jörgen Lindström: Does Distance matter? On geographical dispersion in organisations, 1999, ISBN 917219-439-1.

No 582 Vanja Josifovski: Design, Implementation and Evaluation of a Distributed Mediator System for Data Integration, 1999, ISBN 91-7219-482-0.

No 589 Rita Kovordányi: Modeling and Simulating Inhibitory Mechanisms in Mental Image Reinterpretation - Towards Cooperative HumanComputer Creativity, 1999, ISBN 91-7219-506-1.

No592 Mikael Ericsson: Supporting the Use of Design Knowledge - An Assessment of Commenting Agents, 1999, ISBN 91-7219-532-0.

No 593 Lars Karlsson: Actions, Interactions and Narratives, 1999, ISBN 91-7219-534-7.

No 594 C. G. Mikael Johansson: Social and Organizational Aspects of Requirements Engineering Methods - A practice-oriented approach, 1999, ISBN 91-7219-541$X$.

No 595 Jörgen Hansson: Value-Driven Multi-Class Overload Management in Real-Time Database Systems, 1999, ISBN 91-7219-542-8. 
No 596 Niklas Hallberg: Incorporating User Values in the Design of Information Systems and Services in the Public Sector: A Methods Approach, 1999, ISBN 917219-543-6.

No 597 Vivian Vimarlund: An Economic Perspective on the Analysis of Impacts of Information Technology: From Case Studies in Health-Care towards General Models and Theories, 1999, ISBN 91-7219-544-4.

No 598 Johan Jenvald: Methods and Tools in ComputerSupported Taskforce Training, 1999, ISBN 91-7219547-9.

No 607 Magnus Merkel: Understanding and enhancing translation by parallel text processing, 1999, ISBN 917219-614-9.

No 611 Silvia Coradeschi: Anchoring symbols to sensory data, 1999, ISBN 91-7219-623-8.

No 613 Man Lin: Analysis and Synthesis of Reactive Systems: A Generic Layered Architecture Perspective, 1999, ISBN 91-7219-630-0.

No 618 Jimmy Tjäder: Systemimplementering i praktiken En studie av logiker i fyra projekt, 1999, ISBN 917219-657-2.

No 627 Vadim Engelson: Tools for Design, Interactive Simulation, and Visualization of Object-Oriented Models in Scientific Computing, 2000, ISBN 91-7219709-9.

No 637 Esa Falkenroth: Database Technology for Control and Simulation, 2000, ISBN 91-7219-766-8.

No 639 Per-Arne Persson: Bringing Power and Knowledge Together: Information Systems Design for Autonomy and Control in Command Work, 2000, ISBN 91-7219796-X.

No 660 Erik Larsson: An Integrated System-Level Design for Testability Methodology, 2000, ISBN 91-7219-890-7.

No 688 Marcus Bjäreland: Model-based Execution Monitoring, 2001, ISBN 91-7373-016-5.

No 689 Joakim Gustafsson: Extending Temporal Action Logic, 2001, ISBN 91-7373-017-3.

No 720 Carl-Johan Petri: Organizational Information Provision - Managing Mandatory and Discretionary Use of Information Technology, 2001, ISBN-91-7373-1269.

No 724 Paul Scerri: Designing Agents for Systems with Adjustable Autonomy, 2001, ISBN 9173732079.

No 725 Tim Heyer: Semantic Inspection of Software Artifacts: From Theory to Practice, 2001, ISBN 91 73732087.

No 726 Pär Carlshamre: A Usability Perspective on Requirements Engineering - From Methodology to Product Development, 2001, ISBN 9173732125.

No 732 Juha Takkinen: From Information Management to Task Management in Electronic Mail, 2002, ISBN 91 73732583.

No 745 Johan Åberg: Live Help Systems: An Approach to Intelligent Help for Web Information Systems, 2002, ISBN 91-7373-311-3.

No 746 Rego Granlund: Monitoring Distributed Teamwork Training, 2002, ISBN 91-7373-312-1.

No 757 Henrik André-Jönsson: Indexing Strategies for Time Series Data, 2002, ISBN 917373-346-6.
No 747 Anneli Hagdahl: Development of IT-supported Interorganisational Collaboration - A Case Study in the Swedish Public Sector, 2002, ISBN 91-7373-314-8.

No 749 Sofie Pilemalm: Information Technology for NonProfit Organisations - Extended Participatory Design of an Information System for Trade Union Shop Stewards, 2002, ISBN 91-7373-318-0.

No 765 Stefan Holmlid: Adapting users: Towards a theory of use quality, 2002, ISBN 91-7373-397-0.

No 771 Magnus Morin: Multimedia Representations of Distributed Tactical Operations, 2002, ISBN 91-7373-4217.

No 772 Pawel Pietrzak: A Type-Based Framework for Locating Errors in Constraint Logic Programs, 2002, ISBN 91-7373-422-5.

No 758 Erik Berglund: Library Communication Among Programmers Worldwide, 2002, ISBN 91-7373-349-0.

No 774 Choong-ho Yi: Modelling Object-Oriented Dynamic Systems Using a Logic-Based Framework, 2002, ISBN 91-7373-424-1.

No 779 Mathias Broxvall: A Study in the Computational Complexity of Temporal Reasoning, 2002, ISBN 917373-440-3.

No 793 Asmus Pandikow: A Generic Principle for Enabling Interoperability of Structured and Object-Oriented Analysis and Design Tools, 2002, ISBN 91-7373-479-9.

No 785 Lars Hult: Publika Informationstjänster. En studie av den Internetbaserade encyklopedins bruksegenskaper, 2003, ISBN 91-7373-461-6.

No 800 Lars Taxén: A Framework for the Coordination of Complex Systems' Development, 2003, ISBN 917373-604-X

No 808 Klas Gäre: Tre perspektiv på förväntningar och förändringar i samband med införande av informationssystem, 2003, ISBN 91-7373-618-X.

No 821 Mikael Kindborg: Concurrent Comics programming of social agents by children, 2003, ISBN 91-7373-651-1.

No 823 Christina Ölvingson: On Development of Information Systems with GIS Functionality in Public Health Informatics: A Requirements Engineering Approach, 2003, ISBN 91-7373-656-2.

No 828 Tobias Ritzau: Memory Efficient Hard Real-Time Garbage Collection, 2003, ISBN 91-7373-666-X.

No 833 Paul Pop: Analysis and Synthesis of Communication-Intensive Heterogeneous Real-Time Systems, 2003, ISBN 91-7373-683-X.

No 852 Johan Moe: Observing the Dynamic Behaviour of Large Distributed Systems to Improve Development and Testing - An Empirical Study in Software Engineering, 2003, ISBN 91-7373-779-8.

No 867 Erik Herzog: An Approach to Systems Engineering Tool Data Representation and Exchange, 2004, ISBN 91-7373-929-4.

No 872 Aseel Berglund: Augmenting the Remote Control: Studies in Complex Information Navigation for Digital TV, 2004, ISBN 91-7373-940-5.

No 869 Jo Skåmedal: Telecommuting's Implications on Travel and Travel Patterns, 2004, ISBN 91-7373-935-9. 
No 870 Linda Askenäs: The Roles of IT - Studies of Organising when Implementing and Using Enterprise Systems, 2004, ISBN 91-7373-936-7.

No 874 Annika Flycht-Eriksson: Design and Use of Ontologies in Information-Providing Dialogue Systems, 2004, ISBN 91-7373-947-2.

No 873 Peter Bunus: Debugging Techniques for EquationBased Languages, 2004, ISBN 91-7373-941-3.

No 876 Jonas Mellin: Resource-Predictable and Efficient Monitoring of Events, 2004, ISBN 91-7373-956-1.

No 883 Magnus Bång: Computing at the Speed of Paper: Ubiquitous Computing Environments for Healthcare Professionals, 2004, ISBN 91-7373-971-5

No 882 Robert Eklund: Disfluency in Swedish humanhuman and human-machine travel booking dialogues, 2004, ISBN 91-7373-966-9.

No 887 Anders Lindström: English and other Foreign Linguistic Elements in Spoken Swedish. Studies of Productive Processes and their Modelling using Finite-State Tools, 2004, ISBN 91-7373-981-2.

No 889 Zhiping Wang: Capacity-Constrained Production-inventory systems - Modelling and Analysis in both a traditional and an e-business context, 2004, ISBN 9185295-08-6.

No 893 Pernilla Qvarfordt: Eyes on Multimodal Interaction, 2004, ISBN 91-85295-30-2.

No 910 Magnus Kald: In the Borderland between Strategy and Management Control - Theoretical Framework and Empirical Evidence, 2004, ISBN 91-85295-82-5.

No 918 Jonas Lundberg: Shaping Electronic News: Genre Perspectives on Interaction Design, 2004, ISBN 9185297-14-3.

No 900 Mattias Arvola: Shades of use: The dynamics of interaction design for sociable use, 2004, ISBN 9185295-42-6.

No 920 Luis Alejandro Cortés: Verification and Scheduling Techniques for Real-Time Embedded Systems, 2004, ISBN 91-85297-21-6.

No 929 Diana Szentivanyi: Performance Studies of FaultTolerant Middleware, 2005, ISBN 91-85297-58-5.

No 933 Mikael Cäker: Management Accounting as Constructing and Opposing Customer Focus: Three Case Studies on Management Accounting and Customer Relations, 2005, ISBN 91-85297-64-X.

No 937 Jonas Kvarnström: TALplanner and Other Extensions to Temporal Action Logic, 2005, ISBN 9185297-75-5.

No 938 Bourhane Kadmiry: Fuzzy Gain-Scheduled Visual Servoing for Unmanned Helicopter, 2005, ISBN 9185297-76-3.

No 945 Gert Jervan: Hybrid Built-In Self-Test and Test Generation Techniques for Digital Systems, 2005, ISBN: 91-85297-97-6.

No 946 Anders Arpteg: Intelligent Semi-Structured Information Extraction, 2005, ISBN 91-85297-98-4.

No 947 Ola Angelsmark: Constructing Algorithms for Constraint Satisfaction and Related Problems - Methods and Applications, 2005, ISBN 91-85297-99-2.
No 963 Calin Curescu: Utility-based Optimisation of Resource Allocation for Wireless Networks, 2005, ISBN 91-85457-07-8.

No 972 Björn Johansson: Joint Control in Dynamic Situations, 2005, ISBN 91-85457-31-0.

No 974 Dan Lawesson: An Approach to Diagnosability Analysis for Interacting Finite State Systems, 2005, ISBN 91-85457-39-6.

No 979 Claudiu Duma: Security and Trust Mechanisms for Groups in Distributed Services, 2005, ISBN 91-85457$54-X$.

No 983 Sorin Manolache: Analysis and Optimisation of Real-Time Systems with Stochastic Behaviour, 2005, ISBN 91-85457-60-4.

No 986 Yuxiao Zhao: Standards-Based Application Integration for Business-to-Business Communications, 2005, ISBN 91-85457-66-3.

No 1004 Patrik Haslum: Admissible Heuristics for Automated Planning, 2006, ISBN 91-85497-28-2.

No 1005 Aleksandra Tešanovic: Developing Reusable and Reconfigurable Real-Time Software using Aspects and Components, 2006, ISBN 91-85497-29-0.

No 1008 David Dinka: Role, Identity and Work: Extending the design and development agenda, 2006, ISBN 9185497-42-8.

No 1009 Iakov Nakhimovski: Contributions to the Modeling and Simulation of Mechanical Systems with Detailed Contact Analysis, 2006, ISBN 91-85497-43-X.

No 1013 Wilhelm Dahllöf: Exact Algorithms for Exact Satisfiability Problems, 2006, ISBN 91-85523-97-6.

No 1016 Levon Saldamli: PDEModelica - A High-Level Language for Modeling with Partial Differential Equations, 2006, ISBN 91-85523-84-4.

No 1017 Daniel Karlsson: Verification of Component-based Embedded System Designs, 2006, ISBN 91-85523-79-8

No 1018 Ioan Chisalita: Communication and Networking Techniques for Traffic Safety Systems, 2006, ISBN 9185523-77-1.

No 1019 Tarja Susi: The Puzzle of Social Activity - The Significance of Tools in Cognition and Cooperation, 2006, ISBN 91-85523-71-2.

No 1021 Andrzej Bednarski: Integrated Optimal Code Generation for Digital Signal Processors, 2006, ISBN 9185523-69-0.

No 1022 Peter Aronsson: Automatic Parallelization of Equation-Based Simulation Programs, 2006, ISBN 9185523-68-2.

No 1030 Robert Nilsson: A Mutation-based Framework for Automated Testing of Timeliness, 2006, ISBN 9185523-35-6.

No 1034 Jon Edvardsson: Techniques for Automatic Generation of Tests from Programs and Specifications, 2006, ISBN 91-85523-31-3.

No 1035 Vaida Jakoniene: Integration of Biological Data, 2006, ISBN 91-85523-28-3.

No 1045 Genevieve Gorrell: Generalized Hebbian Algorithms for Dimensionality Reduction in Natural Language Processing, 2006, ISBN 91-85643-88-2. 
No 1051 Yu-Hsing Huang: Having a New Pair of Glasses Applying Systemic Accident Models on Road Safety, 2006, ISBN 91-85643-64-5.

No 1054 Åsa Hedenskog: Perceive those things which cannot be seen - A Cognitive Systems Engineering perspective on requirements management, 2006, ISBN 91-85643-57-2.

No 1061 Cécile Åberg: An Evaluation Platform for Semantic Web Technology, 2007, ISBN 91-85643-31-9.

No 1073 Mats Grindal: Handling Combinatorial Explosion in Software Testing, 2007, ISBN 978-91-85715-74-9.

No 1075 Almut Herzog: Usable Security Policies for Runtime Environments, 2007, ISBN 978-91-85715-65-7.

No 1079 Magnus Wahlström: Algorithms, measures, and upper bounds for Satisfiability and related problems, 2007, ISBN 978-91-85715-55-8.

No 1083 Jesper Andersson: Dynamic Software Architectures, 2007, ISBN 978-91-85715-46-6.

No 1086 Ulf Johansson: Obtaining Accurate and Comprehensible Data Mining Models - An Evolutionary Approach, 2007, ISBN 978-91-85715-34-3.

No 1089 Traian Pop: Analysis and Optimisation of Distributed Embedded Systems with Heterogeneous Scheduling Policies, 2007, ISBN 978-91-85715-27-5.

No 1091 Gustav Nordh: Complexity Dichotomies for CSPrelated Problems, 2007, ISBN 978-91-85715-20-6.

No 1106 Per Ola Kristensson: Discrete and Continuous Shape Writing for Text Entry and Control, 2007, ISBN 97891-85831-77-7.

No 1110 He Tan: Aligning Biomedical Ontologies, 2007, ISBN 978-91-85831-56-2.

No 1112 Jessica Lindblom: Minding the body - Interacting socially through embodied action, 2007, ISBN 978-9185831-48-7.

No 1113 Pontus Wärnestål: Dialogue Behavior Management in Conversational Recommender Systems, 2007, ISBN 978-91-85831-47-0.

No 1120 Thomas Gustafsson: Management of Real-Time Data Consistency and Transient Overloads in Embedded Systems, 2007, ISBN 978-91-85831-33-3.

No 1127 Alexandru Andrei: Energy Efficient and Predictable Design of Real-time Embedded Systems, 2007, ISBN 978-91-85831-06-7.

No 1139 Per Wikberg: Eliciting Knowledge from Experts in Modeling of Complex Systems: Managing Variation and Interactions, 2007, ISBN 978-91-85895-66-3.

No 1143 Mehdi Amirijoo: QoS Control of Real-Time Data Services under Uncertain Workload, 2007, ISBN 97891-85895-49-6.

No 1150 Sanny Syberfeldt: Optimistic Replication with Forward Conflict Resolution in Distributed Real-Time Databases, 2007, ISBN 978-91-85895-27-4.

No 1155 Beatrice Alenljung: Envisioning a Future Decision Support System for Requirements Engineering - A Holistic and Human-centred Perspective, 2008, ISBN 978-91-85895-11-3.

No 1156 Artur Wilk: Types for XML with Application to Xcerpt, 2008, ISBN 978-91-85895-08-3.
No 1183 Adrian Pop: Integrated Model-Driven Development Environments for Equation-Based Object-Oriented Languages, 2008, ISBN 978-91-7393-895-2.

No 1185 Jörgen Skågeby: Gifting Technologies Ethnographic Studies of End-users and Social Media Sharing, 2008, ISBN 978-91-7393-892-1.

No 1187 Imad-Eldin Ali Abugessaisa: Analytical tools and information-sharing methods supporting road safety organizations, 2008, ISBN 978-91-7393-887-7.

No 1204 H. Joe Steinhauer: A Representation Scheme for Description and Reconstruction of Object Configurations Based on Qualitative Relations, 2008, ISBN 978-91-7393-823-5.

No 1222 Anders Larsson: Test Optimization for Core-based System-on-Chip, 2008, ISBN 978-91-7393-768-9.

No 1238 Andreas Borg: Processes and Models for Capacity Requirements in Telecommunication Systems, 2009, ISBN 978-91-7393-700-9.

No 1240 Fredrik Heintz: DyKnow: A Stream-Based Knowledge Processing Middleware Framework, 2009, ISBN 978-91-7393-696-5.

No 1241 Birgitta Lindström: Testability of Dynamic RealTime Systems, 2009, ISBN 978-91-7393-695-8.

No 1244 Eva Blomqvist: Semi-automatic Ontology Construction based on Patterns, 2009, ISBN 978-91-7393-683-5.

No 1249 Rogier Woltjer: Functional Modeling of Constraint Management in Aviation Safety and Command and Control, 2009, ISBN 978-91-7393-659-0.

No 1260 Gianpaolo Conte: Vision-Based Localization and Guidance for Unmanned Aerial Vehicles, 2009, ISBN 978-91-7393-603-3.

No 1262 AnnMarie Ericsson: Enabling Tool Support for Formal Analysis of ECA Rules, 2009, ISBN 978-91-7393598-2.

No 1266 Jiri Trnka: Exploring Tactical Command and Control: A Role-Playing Simulation Approach, 2009, ISBN 978-91-7393-571-5.

No 1268 Bahlol Rahimi: Supporting Collaborative Work through ICT - How End-users Think of and Adopt Integrated Health Information Systems, 2009, ISBN 978-91-7393-550-0.

No 1274 Fredrik Kuivinen: Algorithms and Hardness Results for Some Valued CSPs, 2009, ISBN 978-91-7393-525-8.

No 1281 Gunnar Mathiason: Virtual Full Replication for Scalable Distributed Real-Time Databases, 2009, ISBN 978-91-7393-503-6.

No 1290 Viacheslav Izosimov: Scheduling and Optimization of Fault-Tolerant Distributed Embedded Systems, 2009, ISBN 978-91-7393-482-4.

No 1294 Johan Thapper: Aspects of a Constraint Optimisation Problem, 2010, ISBN 978-91-7393-464-0.

No 1306 Susanna Nilsson: Augmentation in the Wild: User Centered Development and Evaluation of Augmented Reality Applications, 2010, ISBN 978-917393-416-9.

No 1313 Christer Thörn: On the Quality of Feature Models, 2010, ISBN 978-91-7393-394-0.

No 1321 Zhiyuan He: Temperature Aware and DefectProbability Driven Test Scheduling for System-onChip, 2010, ISBN 978-91-7393-378-0. 
No 1333 David Broman: Meta-Languages and Semantics for Equation-Based Modeling and Simulation, 2010, ISBN 978-91-7393-335-3.

No 1337 Alexander Siemers: Contributions to Modelling and Visualisation of Multibody Systems Simulations with Detailed Contact Analysis, 2010, ISBN 978-91-7393317-9.

No 1354 Mikael Asplund: Disconnected Discoveries: Availability Studies in Partitioned Networks, 2010, ISBN 978-91-7393-278-3.

No 1359 Jana Rambusch: Mind Games Extended: Understanding Gameplay as Situated Activity, 2010, ISBN 978-91-7393-252-3.

No 1373 Sonia Sangari: Head Movement Correlates to Focus Assignment in Swedish,2011,ISBN 978-91-7393-154-0.

No 1374 Jan-Erik Källhammer: Using False Alarms when Developing Automotive Active Safety Systems, 2011, ISBN 978-91-7393-153-3.

No 1375 Mattias Eriksson: Integrated Code Generation, 2011, ISBN 978-91-7393-147-2.

No 1381 Ola Leifler: Affordances and Constraints of Intelligent Decision Support for Military Command and Control - Three Case Studies of Support Systems, 2011, ISBN 978-91-7393-133-5.

No 1386 Soheil Samii: Quality-Driven Synthesis and Optimization of Embedded Control Systems, 2011, ISBN 978-91-7393-102-1.

No 1419 Erik Kuiper: Geographic Routing in Intermittentlyconnected Mobile Ad Hoc Networks: Algorithms and Performance Models, 2012, ISBN 978-91-7519981-8.

No 1451 Sara Stymne: Text Harmonization Strategies for Phrase-Based Statistical Machine Translation, 2012, ISBN 978-91-7519-887-3.

No 1455 Alberto Montebelli: Modeling the Role of Energy Management in Embodied Cognition, 2012, ISBN 978-91-7519-882-8.

No 1465 Mohammad Saifullah: Biologically-Based Interactive Neural Network Models for Visual Attention and Object Recognition, 2012, ISBN 978-91-7519-838-5.

No 1490 Tomas Bengtsson: Testing and Logic Optimization Techniques for Systems on Chip, 2012, ISBN 978-917519-742-5.

No 1481 David Byers: Improving Software Security by Preventing Known Vulnerabilities, 2012, ISBN 97891-7519-784-5.

No 1496 Tommy Färnqvist: Exploiting Structure in CSPrelated Problems, 2013, ISBN 978-91-7519-711-1.

No 1503 John Wilander: Contributions to Specification, Implementation, and Execution of Secure Software, 2013, ISBN 978-91-7519-681-7.

No 1506 Magnus Ingmarsson: Creating and Enabling the Useful Service Discovery Experience, 2013, ISBN 97891-7519-662-6.

No 1547 Wladimir Schamai: Model-Based Verification of Dynamic System Behavior against Requirements: Method, Language, and Tool, 2013, ISBN 978-917519-505-6.

No 1551 Henrik Svensson: Simulations, 2013, ISBN 978-917519-491-2.
No 1559 Sergiu Rafiliu: Stability of Adaptive Distributed Real-Time Systems with Dynamic Resource Management, 2013, ISBN 978-91-7519-471-4.

No 1581 Usman Dastgeer: Performance-aware Component Composition for GPU-based Systems, 2014, ISBN 978-91-7519-383-0.

No 1602 Cai Li: Reinforcement Learning of Locomotion based on Central Pattern Generators, 2014, ISBN 978-917519-313-7.

No 1652 Roland Samlaus: An Integrated Development Environment with Enhanced Domain-Specific Interactive Model Validation, 2015, ISBN 978-917519-090-7.

No 1663 Hannes Uppman: On Some Combinatorial Optimization Problems: Algorithms and Complexity, 2015, ISBN 978-91-7519-072-3.

No 1664 Martin Sjölund: Tools and Methods for Analysis, Debugging, and Performance Improvement of Equation-Based Models, 2015, ISBN 978-91-7519-071-6.

No 1666 Kristian Stavåker: Contributions to Simulation of Modelica Models on Data-Parallel Multi-Core Architectures, 2015, ISBN 978-91-7519-068-6.

No 1680 Adrian Lifa: Hardware/Software Codesign of Embedded Systems with Reconfigurable and Heterogeneous Platforms, 2015, ISBN 978-91-7519-0402.

No 1685 Bogdan Tanasa: Timing Analysis of Distributed Embedded Systems with Stochastic Workload and Reliability Constraints, 2015, ISBN 978-91-7519-022-8.

No 1691 Håkan Warnquist: Troubleshooting Trucks Automated Planning and Diagnosis, 2015, ISBN 97891-7685-993-3.

No 1702 Nima Aghaee: Thermal Issues in Testing of Advanced Systems on Chip, 2015, ISBN 978-91-7685949-0.

\section{Linköping Studies in Arts and Science}

No 504 Ing-Marie Jonsson: Social and Emotional Characteristics of Speech-based In-Vehicle Information Systems: Impact on Attitude and Driving Behaviour, 2009, ISBN 978-91-7393-478-7.

No 586 Fabian Segelström: Stakeholder Engagement for Service Design: How service designers identify and communicate insights, 2013, ISBN 978-91-7519-554-4.

No 618 Johan Blomkvist: Representing Future Situations of Service: Prototyping in Service Design, 2014, ISBN 978-91-7519-343-4.

No 620 Marcus Mast: Human-Robot Interaction for SemiAutonomous Assistive Robots, 2014, ISBN 978-917519-319-9.

\section{Linköping Studies in Statistics}

No 9 Davood Shahsavani: Computer Experiments Designed to Explore and Approximate Complex Deterministic Models, 2008, ISBN 978-91-7393-976-8.

No 10 Karl Wahlin: Roadmap for Trend Detection and Assessment of Data Quality, 2008, ISBN 978-91-7393$792-4$. 
No 11 Oleg Sysoev: Monotonic regression for large multivariate datasets, 2010, ISBN 978-91-7393-412-1.

No 13 Agné Burauskaite-Harju: Characterizing Temporal Change and Inter-Site Correlations in Daily and Subdaily Precipitation Extremes, 2011, ISBN 978-91-7393110-6.

\section{Linköping Studies in Information Science}

No 1 Karin Axelsson: Metodisk systemstrukturering- att skapa samstämmighet mellan informationssystemarkitektur och verksamhet, 1998. ISBN-9172-19-296-8.

No 2 Stefan Cronholm: Metodverktyg och användbarhet en studie av datorstödd metodbaserad systemutveckling, 1998, ISBN-9172-19-299-2.

No 3 Anders Avdic: Användare och utvecklare - om anveckling med kalkylprogram, 1999. ISBN-91-7219606-8.

No 4 Owen Eriksson: Kommunikationskvalitet hos informationssystem och affärsprocesser, 2000, ISBN 917219-811-7.

No 5 Mikael Lind: Från system till process - kriterier för processbestämning vid verksamhetsanalys, 2001, ISBN 91-7373-067-X.

No 6 Ulf Melin: Koordination och informationssystem i företag och nätverk, 2002, ISBN 91-7373-278-8.

No 7 Pär J. Ågerfalk: Information Systems Actability - Understanding Information Technology as a Tool for Business Action and Communication, 2003, ISBN 917373-628-7.

No 8 Ulf Seigerroth: Att förstå och förändra systemutvecklingsverksamheter - en taxonomi för metautveckling, 2003, ISBN91-7373-736-4.

No 9 Karin Hedström: Spår av datoriseringens värden Effekter av IT i äldreomsorg, 2004, ISBN 91-7373-9634.

No 10 Ewa Braf: Knowledge Demanded for Action Studies on Knowledge Mediation in Organisations, 2004, ISBN 91-85295-47-7.

No 11 Fredrik Karlsson: Method Configuration method and computerized tool support, 2005, ISBN 91-8529748-8.

No 12 Malin Nordström: Styrbar systemförvaltning - Att organisera systemförvaltningsverksamhet med hjälp av effektiva förvaltningsobjekt, 2005, ISBN 91-8529760-7.

No 13 Stefan Holgersson: Yrke: POLIS - Yrkeskunskap, motivation, IT-system och andra förutsättningar för polisarbete, 2005, ISBN 91-85299-43-X.

No 14 Benneth Christiansson, Marie-Therese Christiansson: Mötet mellan process och komponent - mot ett ramverk för en verksamhetsnära kravspecifikation vid anskaffning av komponentbaserade informationssystem, 2006, ISBN 91-85643$22-X$. 